\title{
A catalog of stellar magnetic rotational phase curves ${ }^{\star}$
}

\author{
V. D. Bychkov ${ }^{1,2}$, L. V. Bychkova ${ }^{1}$, and J. Madej ${ }^{3}$ \\ ${ }^{1}$ Special Astrophysical Observatory of the Russian Academy of Sciences, Nizhnij Arkhyz, 369167 Russia \\ e-mail: vbych@sao.ru \\ 2 Isaac Newton Institute of Chile, SAO Branch, Nizhnij Arkhyz, 369167 Russia \\ 3 Astronomical Observatory, University of Warsaw, Al. Ujazdowskie 4, 00-478 Warszawa, Poland
}

Received 22 October 2003 / Accepted 20 September 2004

\begin{abstract}
Magnetized stars usually exhibit periodic variations of the effective (longitudinal) magnetic field $B_{\mathrm{e}}$ caused by their rotation. We present a catalog of magnetic rotational phase curves, $B_{\mathrm{e}}$ vs. the rotational phase $\phi$, and tables of their parameters for 136 stars on the main sequence and above it. Phase curves were obtained by the least squares fitting of sine wave or double wave functions to the available $B_{\mathrm{e}}$ measurements, which were compiled from the existing literature. Most of the catalogued objects are chemically peculiar A and B type stars (127 stars). For some stars we also improved or determined periods of their rotation. We discuss the distribution of parameters describing magnetic rotational phase curves in our sample.
\end{abstract}

Key words. catalogs - stars: fundamental parameters - stars: magnetic fields - stars: rotation

\section{Introduction}

Strong global magnetic fields can be found in most chemically peculiar $(\mathrm{CP})$ stars on the upper main sequence of the Hertzsprung-Russell diagram. These stars are usually referred to as Ap or Bp stars, depending on the given effective temperature, and hence the principal spectral class. Magnetic fields in CP stars were discovered and have been measured for a long time. However, global magnetic fields also can be found in stars of other spectral types.

There exist claims on the existence of global-scale magnetic fields in some pulsating stars (Weiss \& Wood 1975; Borra et al. 1981; Romanov et al. 1985; Weiss 1986; Neiner et al. 2003, for instance), in some close binary systems (Skulskij et al. 1992a,b), and in a few supergiants (Rakos et al. 1977; Scholz \& Gerth 1980; Weiss 1986; Gerth 1988; Plachinda 1990; Gerth et al. 1991) and exceptionally in solar-like stars (Plachinda \& Tarasova 1999, 2000b; Tarasova et al. 2001; Plachinda et al. 2001). Global magnetic fields are also observed in Be stars (Henrichs et al. 2000; Donati et al. 2001).

The above discoveries have been followed by recent high accuracy research programs (Wade et al. 2002; Chadid et al. 2003), but still require more $B_{\mathrm{e}}$ measurements and observational verification.

Most late type stars on the main sequence have local magnetic fields, which are restricted to relatively small active regions on the surface (Robinson et al. 1980; Saar 1988). Despite a few exceptions, global magnetic fields generally do not exist

* All tables and Appendix A are only available in electronic form at http://www.edpsciences.org in that group of stars (Borra et al. 1981; Brown \& Landstreet 1987). Also, highly evolved stars on the horizontal branch of giants do not have global-scale magnetic fields (El'kin 1992).

Recently, Smirnov et al. (2003a,b) have found magnetic fields in a few $\mathrm{T}$ Tau stars. This discovery, however, requires independent confirmation.

The most useful observable global quantity is the effective (longitudinal) magnetic field $B_{\mathrm{e}}$, which is observed through Zeeman splitting of some spectral lines into pairs of circularly polarised components. The effective magnetic field $B_{\mathrm{e}}$, or rather its quadratic average $\left\langle B_{\mathrm{e}}\right\rangle$ over many individual observations, can yield the averaged global-scale surface magnetic field strength of an individual star.

A definition of the quadratic effective magnetic field $\left\langle B_{\mathrm{e}}\right\rangle$ was given by Borra et al. (1983). Extensive tables of $\left\langle B_{\mathrm{e}}\right\rangle$ were recently compiled by Bychkov et al. (2003). They discussed also the distribution and other properties of $\left\langle B_{\mathrm{e}}\right\rangle$ in various subclasses of magnetic Ap stars, with 352 Ap and Bp stars.

\subsection{Magnetism of CP stars}

A substantial number of magnetic $\mathrm{CP}$ stars exhibit periodic variations of their effective magnetic fields $B_{\mathrm{e}}$ with their rotational period. Periods of $B_{\mathrm{e}}$ variations of CP stars most often are in the range of 2-5 days (Lebedev 1986; Renson \& Catalano 2001). In most cases the dependence of $B_{\mathrm{e}}$ on the rotational phase $\phi$ exhibits a simple sine wave form. Such a variability is very well described in frame by the Stibbs-Preston oblique rotator model (Stibbs 1950; Preston 1967a). In that model, the dipole magnetic field is stationary, i.e. the dipole 
does not change its parameters with time. Both the star and the dipole rotate about the rotational axis with the same angular velocity. Periodic variability of $B_{\mathrm{e}}$ is directly caused by the rotation which modulates the projection of $B_{\mathrm{e}}$ in the direction towards the Earth, see Eq. (1).

Some of the magnetic CP stars exhibit more complicated rotational phase variations of $B_{\mathrm{e}}$, which are interpreted as evidence that surface field configurations are more complex than a simple dipole. Stellar rotation can also cause apparent periodic variations of the longitudinal component of the global scale (integrated) magnetic field in the few late-type stars where it has been detected.

On the contrary, there exists evidence that the magnetic variability of long-period Ap stars is caused by the intrinsic variability of their magnetic fields, and not by stellar rotation. Such a mechanism could be similar to the solar cycle (Krause \& Scholz 1981) in agreement with the dynamo model proposed by Steenbeck \& Krause (1968). Therefore, one cannot claim with confidence that the $B_{\mathrm{e}}$ variability of e.g. $\gamma$ Equ (F0 $\mathrm{SrCrEu}$ ) or similar stars is necessarily due to stellar rotation and the related changes of aspect. Also some of the colder CP stars exhibit "secular" changes of their magnetic field $B_{\mathrm{e}}$ (Bonsack et al. 1974; Scholz 1979; Bychkov \& Shtol' 1997).

\subsection{Observational data}

Variability of the effective magnetic fields $B_{\mathrm{e}}$ in CP stars was discovered over 50 years ago (Babcock \& Burd 1952). A large amount of observational data has been collected in the literature. However, research programs investigating magnetic variability of stars with the rotational phase $\phi$ usually presented observations of a few magnetic stars per paper at most. This is obviously due to the fact that acquiring of even a single magnetic phase curve $B_{\mathrm{e}}(\phi)$ of a star requires much effort and observing time. Therefore, available $B_{\mathrm{e}}$ rotational phase curves are either scattered in the literature, or are presented in an inhomogeneous form, or are incomplete.

We performed an extensive search of the literature and have compiled a catalogue of all the available series of $B_{\mathrm{e}}$ measurements and their magnetic rotational phase curves $B_{\mathrm{e}}(\phi)$. In this paper the catalogue of magnetic rotational phase curves is expressed in a uniform way. Curves $B_{\mathrm{e}}(\phi)$ are fitted either by a simple sine function, or by a double wave (for 18 stars). In some cases we determined new values of the magnetic periods, assuming that $P_{\text {mag }}=P_{\text {rot }}$. Our catalogue also lists parameters of the magnetic rotational phase curves.

Our catalog of stellar magnetic rotational phase curves is very strongly dominated by Ap stars, which make up over $93 \%$ of the sample. Such a bias was caused by a relatively low accuracy of $B_{\mathrm{e}}$ measurements in the past. Consequently, the observer's interest was directed towards stars with strong magnetic fields. The recent development of high accuracy observational techniques allowed several teams to discover and measure global magnetic fields also in a few solar-type stars and in high luminosity stars. The nature of the magnetic activity in both types of stars differs strongly from the magnetism of Ap stars. However, disregarding their different origin we could produce magnetic rotational phase curves for all the above classes of stars with the available $B_{\mathrm{e}}$ measurements. We can also anticipate that the number of stars other than $\mathrm{CP}$ stars with measured global-scale longitudinal magnetic fields will rise soon. Thus, we do not restrict our paper to CP stars, and present here also several magnetic rotational phase curves for stars of other types.

Analysis of the catalogue enabled us to select stars for which additional $B_{\mathrm{e}}$ observations are most urgently needed, or those stars in which apparent $B_{\mathrm{e}}$ variations are particularly complex or suggest secular changes not necessarily correlated with the rotation.

\subsection{Completeness of the catalog}

This paper presents the first catalog of magnetic rotational phase curves. We attempted to compile magnetic phase curves from $B_{\mathrm{e}}$ observations retrieved from different sources, determine the new or consistent magnetic (rotational) period, where appropriate, and present the resulting magnetic phase curves and parameters of magnetic variability in a uniform manner.

The catalog contains magnetic phase curves for all stars for which the number of published $B_{\mathrm{e}}$ points is sufficiently high to construct a phase curve. In such a way the catalog is complete. On the other hand, it reflects the extreme incompleteness of the available observational data, in which magnetic observations of CP stars strongly dominate the whole set of the available data. In this aspect our catalog is missing many stars of other spectral types, e.g. late-type stars, which on average exhibit much weaker and sparsely investigated global magnetic fields.

\section{Effective (longitudinal) magnetic fields}

The effective field $B_{\mathrm{e}}$ is the observable magnetic quantity, determined by the Zeeman splitting of selected lines in stellar spectra. In the case of a spherical star, it is the average of the projected surface field $\boldsymbol{B}_{\text {loc }}$ over the visible stellar disc, weighted by the local monochromatic intensity $I_{v}$ of outgoing radiation

$$
B_{\mathrm{e}}=\frac{\int_{0}^{2 \pi} \int_{0}^{\pi / 2} B_{\mathrm{loc}} \cos \gamma I_{\nu}(\theta) \sin \theta \cos \theta \mathrm{d} \theta \mathrm{d} \varphi}{\int_{0}^{2 \pi} \int_{0}^{\pi / 2} I_{\nu}(\theta) \sin \theta \cos \theta \mathrm{d} \theta \mathrm{d} \varphi},
$$

where $\gamma$ denotes the angle between the local vector $\boldsymbol{B}_{\text {loc }}$ and the direction towards the observer. The variable $\theta$ denotes the colatitude angle, and $\varphi$ is the azimuthal angle of the angular integration. The above definition implicitly assumes that $B_{\mathrm{e}}$ is determined at a single discrete frequency only (Madej 1983; Bychkov et al. 2003).

The scalar variable $B_{\mathrm{e}}$ is directly observable, and can be measured by the amount of splitting of selected spectral lines into circularly polarised components. Zeeman splitting is caused by the shift of energy levels of atomic spin states. The effect is best observed in atoms with single unpaired electron, 
$s=1 / 2$, which occurs in hydrogen $\mathrm{H} \mathrm{I}$, singly ionized iron Fe II, and also in highly excited He I.

Equation (1) implies that $B_{\mathrm{e}}$ is determined both by the intrinsic configuration and strength of the stellar magnetic field $\boldsymbol{B}$, and by its momentary projection in the direction towards the Earth. Therefore, if the intrinsically static surface magnetic field is not aligned with the rotation axis, then the star has to exhibit periodic $B_{\mathrm{e}}$ variations with the period $P_{\mathrm{mag}}$ equal to the period of rotation, $P_{\text {rot }}$. In particular, this is true for the oblique dipole model of a stellar magnetic field. The effective magnetic field $B_{\mathrm{e}}$ can also change sign during rotation of a star.

\section{Averaged magnetic rotational phase curves}

We have studied periodic variations of the effective magnetic field $B_{\mathrm{e}}$ with the rotational phase $\phi$ in the following manner.

1. For all stars with an adequate number of $B_{\mathrm{e}}$ determinations, and for which the period of magnetic variability $P_{\text {mag }}$ was known, we have determined the best fit for the relation of $B_{\mathrm{e}} \mathrm{vs}$. phase

$B_{\mathrm{e} i}(\phi)=B_{0}+B_{1} \cos \phi$,

where

$\phi=2 \pi\left(\frac{T_{i}-T_{0}}{P}\right)$,

by the least squares method. Here $B_{0}$ is the average field, $B_{1}$ stands for half of the amplitude, $T_{i}$ is time of measurement, $P$ denotes the period, and $T_{0}$ is the zero epoch, i.e. time corresponding to the zero phase $\phi$. We have chosen the zero epoch $T_{0}$ in such a way that the phase $\phi=0$ corresponds to the minimum of the best fit magnetic curve.

In the following Tables 1-3 we present listing of parameters of the magnetic curves, and also the parameter $r$, which was defined by Stibbs (1950). Parameter $r$ relates both the angle $\beta$ between the magnetic dipole axis and the rotational axis, and the angle $i$ between rotational axis and the line of sight

$r=\frac{\cos \beta \cos i-\sin \beta \sin i}{\cos \beta \cos i+\sin \beta \sin i}$.

Alternatively, one can write

$r=\frac{B_{\mathrm{e}}(\min )}{B_{\mathrm{e}}(\max )}$.

2. There are stars with unknown periods of magnetic variations. In some cases if we were able to compile a sufficient number of momentary $B_{\mathrm{e}}$ measurements from the available papers, then we have attempted also to search for the magnetic period $P$ with standard methods. We are aware that periods determined in those cases require additional $B_{\mathrm{e}}$ measurements, or new spectral, photometric, and polarimetric observations in order to confirm our magnetic periods and to decrease their errors.

3. In the case when the shape of the magnetic phases curve is more complex than a simple cosine, we include the second harmonic wave

$B_{\mathrm{e} i}(\phi)=B_{0}+B_{1} \cos \left(\phi+z_{1}\right)+B_{2} \cos \left(2 \phi+z_{2}\right)$.
4. There are also exceptional stars, which exhibit still more complex magnetic phase curves, and in such cases Eq. (6) does not provide an adequate fit. A very good example of that is the star HD 37776, which exhibits a magnetic rotational phase curve of exceptional complexity. For this star we present the fitting curve of other forms, and regard that star as a completely individual case.

\section{Error analysis}

For each star in the sample we performed a $\chi^{2}$ test for evaluation of the goodness of the assumed fit, given either by Eq. (2) or Eq. (6), and the estimated scatter of the available $B_{\mathrm{e}}$ measurements.

A statistical $\chi^{2}$ test can indicate a large discrepancy between observed points and the assumed fitting curve if either the fitting curve is intrinsically inconsistent with observations, or errors of observations (i.e. values of $B_{\mathrm{e}}$ ) are overestimated.

Error estimates of all parameters $T_{0}, B_{0}, B_{1}, B_{2}$, and $r$ were performed in the following way. For each $B_{\mathrm{e} i}$ measurement with known standard error $\sigma_{i}$ we generated a series of 1000 or even more secondary $B_{\mathrm{e} i}^{\mathrm{sec}}$ values with a random number generator. Values of $B_{\mathrm{e} i}^{\mathrm{sec}}$ had an assumed normal distribution around the observed $B_{\mathrm{e} i}$ with the width $\sigma_{i}$. In the case where the authors did not provide a $\sigma_{i}$ estimate, we used an arbitrary error typical for the given method of observation.

Each observed $B_{\mathrm{e} i}$ value was then replaced by a single $B_{\mathrm{e} i}^{\mathrm{sec}}$ chosen randomly from the precomputed artificial series. Then the set of secondary parameters $T_{0}^{\mathrm{sec}}, B_{0}^{\mathrm{sec}}, B_{1}^{\mathrm{sec}}, B_{2}^{\mathrm{sec}}$, and $r^{\mathrm{sec}}$ was determined.

The above computations were repeated many times (usually 1000 times or more). In such a way we obtained numerous sets of secondary fitting parameters, and were able to estimate errors of real $T_{0}, B_{0}, B_{1}, B_{2}$, and $r$ separately.

\section{Methods for the measurement of stellar effective magnetic fields}

In this section we describe the principal methods of $B_{\mathrm{e}}$ measurements.

1. The photographic method is the earliest technique of the magnetic field measurements, which allowed Babcock (1952) to discover magnetic fields of Ap stars. An analyser of circular polarisation is placed in front of the spectrograph slit. Then, the radiation beam from a star is split into two beams, which are circularly polarised in either the clockwise or counterclockwise direction of rotation, and recorded on a photographic plate. Such a method allows one to observe simultaneously two spectra of a star in two opposite states of circular polarisation, and to observe $\sigma_{+}$and $\sigma_{-}$components of lines split by the Zeeman effect.

Strictly speaking, the method is to measure the Zeeman splitting of resolved sigma components, and photographic plates are used to record the spectra.

Measuring the displacement between both components of a spectral line allows one to estimate the longitudinal component of the magnetic field integrated over the line forming region (or the whole stellar disc). Such an estimate is possible if the 
necessary parameters of a given line, including Landè factors, are known.

The accuracy of the above method of $B_{\mathrm{e}}$ observations is low. The accuracy is strongly limited by the width of a line and altered most by the projected velocity of rotation $v \sin i$. For example, if $v \sin i>35 \mathrm{~km} \mathrm{~s}^{-1}$, the number of measured lines $N<30$, and spectral resolution $R<20000$ (dispersion $\geq$ $10 \AA / \mathrm{mm}$ ), then the standard error of $B_{\mathrm{e}}$ is larger than the value of $B_{\mathrm{e}}$ itself. In such a case the measurement of $B_{\mathrm{e}}$ is indefinite.

Magnetic field measurements are commonly performed in metal lines. As is well known, metals are distributed nonuniformly over the surface of an Ap star, and such an inhomogeneity causes additional distortion in estimates of $B_{\mathrm{e}}$ integrated over the stellar surface. In spite of that, the above method allowed astrophysicists to obtain the first measurements of stellar longitudinal magnetic fields. The method was described in detail in Babcock (1958) and Glagolevskij et al. (1977).

2. Hl: effective magnetic fields are measured from the degree of circular polarisation in the wings of hydrogen Balmer lines (Angel \& Landstreet 1970; Landstreet 1982; Shtol' et al. 1985). This method has a few important advantages. First, the accuracy of measurement does not depend on the projected rotational rate $v \sin i$. Second, measurements of $B_{\mathrm{e}}$ are not affected by the details of inhomogeneous distribution of chemical elements on the stellar surface. Therefore, $B_{\mathrm{e}}$ measurements obtained in this way are considered as the most correct magnetic data and best suited for calibration purposes.

3. Scanning of a spectral line was the first photoelectric metod used to measure stellar magnetic fields (Severny 1970; Borra \& Landstreet 1973; Borra \& Vaughan 1977). A one-channel phototube is located in the focal point of the camera of a high resolution spectrograph. The device measures the opposite circularly polarised radiation in a very narrow spectral bin of $0.1-0.2 \AA$. After that the same measurements are performed in neighbouring bins, until one gets the scan of flux and degree of circular polarisation across the whole profile of a given metal line. The estimate of $B_{\mathrm{e}}$ results from a comparison between the circular polarisation integrated over the short wavelength and long wavelength wings of that line.

The advantage of this method is that it yields a reliable estimate of the longitudinal magnetic field $B_{\mathrm{e}}$, particularly for lines with a complex profile. The disadvantage is that the method requires a large number of measurements in the line profile, which decreases the effectiveness of observations.

The "differential" version of the above method makes use of selected points in line wings to estimate $B_{\mathrm{e}}$. Such a method is a faster and more effective way of $B_{\mathrm{e}}$ determination, which requires fewer points in the line profile. On the other hand, the estimated value of $B_{\mathrm{e}}$ strongly depends of the value of $v \sin i$ (Piskunov \& Khohlova 1983).

Thus, the scanning line methods outlined above allow for $B_{\mathrm{e}}$ observations of high accuracy. However, in both methods all measurements are usually performed on a single line of the particular element. One can expect negative effects of line blending and the inhomogeneous distribution of that element over the stellar surface.

The method was extended by Borra et al. (1981). They used a special mask to scan $\approx 230$ lines simultaneously to obtain the averaged line profile and the distribution of circular polarisation in the profile.

4. The multiline Zeeman polarimetric technique (Brown \& Landstreet 1981). The observer uses the same masks used for the usual measurements of radial velocity, and measures radial velocities in spectra circularly polarised in opposite directions. The difference between the radial velocities, averaged over all the measured lines, is the measure of $B_{\mathrm{e}}$.

This approach requires use of at least one hundred lines, which should be narrow and sharp. Moreover, the determination of $B_{\mathrm{e}}$ is done by averaging of the Zeeman splitting amplitude over many lines ignoring their individual intensities and magnetic sensitivity etc. This method was used for $B_{\mathrm{e}}$ measurements in cool stars with narrow spectral lines.

A similar method was used for the measurement of photographic spectra with roughly the same advantages and disadvantages (Weiss et al. 1978).

5. CCD method. This is the method of acquiring of Zeeman spectra by CCD detectors. This technique is very similar to the classical photographic method. The essential difference is that the splitting of sigma components of metal lines or in the wing of Balmer line is recorded digitally with very high positional and photometric accuracy. As a result one can apply a variety of image processing methods which eventually increase the accuracy of the $B_{\mathrm{e}}$ determinations. For example, CCD detectors register echelle type high dispersion spectra, with bent shape orders. Such distorted spectra can be easily processed with the standard data reduction software (MIDAS, IRAF etc.).

Today CCD are used in place of photographic plates or single- or multi-channel phototubes.

6. Least-Squares Deconvolution (LSD), cf. Donati et al. (1997) and its extension, the WLSD method (this is the LSD method improved by Wade et al. 2000c) are the most reliable methods of CCD Zeeman spectra reduction. A spectral line plus surrounding continuum observed by the CCD are represented by a 2-dimensional rectangular $(n, m)$ matrix and each pixel in the matrix is assigned a value equal to the number of photons accumulated in that CCD pixel. Then the observed matrix is best fitted by the "model" line profile converted to the same form. In such a way the effective determination of the best fit of theoretical to the observational line profile reduces to standard tasks of linear algebra. Both methods of data reduction make use of all the information contained in a given spectral line.

There are a number of other methods for the measurement of stellar magnetic fields. such as

- scanning of a spectral line with a Fabry-Perot interferometer (Met) and measuring of the $(V, I)$ Stokes parameters in polarised light (Borra \& Vaughan 1977, 1978; Glagolevskij et al. 1979);

- the Robinson method for measuring of the surface magnetic field in late type stars (Robinson 1980; Robinson et al. 1980; Saar 1988);

- curve of growth method, which makes use of the magnetic intensification of lines (Hensberge \& De Loore 1974; Kolev 1977); 
- measuring of the surface magnetic fields in split lines (Preston 1969a, 1970, 1971; Wolff \& Wolff 1970; Huchra 1972; Borra \& Landstreet 1977; Landstreet 1988; Mathys \& Lanz 1992);

- measuring of the so-called $\lambda^{2}$ effect (Lebedev 1990).

Methods that make use of metal lines will be referred to as (Met), and methods that make use of hydrogen lines will be referred to as $(\mathrm{Hl})$. We have presented here only the most important and widely used methods of measuring the longitudinal magnetic field, $B_{\mathrm{e}}$.

\section{Tabular data}

A full catalog of investigated stars and the corresponding parameters of the best fit sine wave magnetic curves, Eq. (2), is presented in Table 1. Columns of Table 1 list: HD number, spectral type, $B_{0}, \sigma_{B_{0}}, B_{1}, \sigma_{B_{1}}$ (in G), period $P, T_{0}$, and $\sigma_{T_{0}}$ (in days).

Here $B_{0}$ is the average field, $B_{1}$ stands for half of the amplitude, and $T_{0}$ is the zero epoch, i.e. the date corresponding to the zero phase $\phi$. We have chosen the zero epoch $T_{0}$ in such a way that the phase $\phi=0$ corresponds to the minimum of the best fit magnetic curve for all listed stars. Julian Day of the zero phase is $\mathrm{JD}=2400000 .+T_{0}$.

Table 2 of our catalog presents for each star: HD number, $N$ - number of individual points, $\sigma$ - average scatter of $B_{\mathrm{e} i}$ observation around the fitting curve, parameter $r$ and its standard error $\sigma_{r}, N_{V}=N-3$ (for sine wave fits), $\chi^{2}$ for one degree of freedom, method of measurement, reference numbers and (in some cases) the running number of brief comments.

There are 18 magnetic stars that display more complex phase curves $B_{\mathrm{e}}(\phi)$. For these stars phase curves were fitted by the expansion in a harmonic series with the second cosine term, see Eq. (6) (double wave).

Table 3 presents additional parameters necessary to define the double wave. These are coefficients $B_{2}$, phase shifts $z_{1}$ and $z_{2}$, and their errors $\sigma_{B_{2}}, \sigma_{z_{1}}, \sigma_{z_{2}}, N_{V}=N-5$, values of $\chi^{2}$, number of references and comments.

Table 4 lists cross-reference numbers for papers with observational data.

Table 5 presents a compilation of the periods used in this paper. Most of them are rotational periods of the catalogued stars. Entries are: HD number, period (in days) and the exact reference. Table 5 clearly indicates stars for which the rotational period was determined in this paper.

\section{Comments on individual stars}

\subsection{Ap and Bp stars}

$H D 8441$. No precise value of the period was available in the literature. Estimates of the period ranged from 2 to 100 days (Catalano \& Renson 1998).

HD 9996. Preston \& Wolf (1970) and Scholz (1983) suggested the existence of a magnetic period in the range 8000-8400 days. The best period $P_{\mathrm{mag}}$ for $B_{\mathrm{e}}$ measurement that we collected here equals $7692^{\mathrm{d}}$. It is necessary to continue monitoring of this star to determine a more accurate period and magnetic phase behaviour.

HD 35456. Borra (1981) presents 4 different possible values of the period of the projected magnetic field in the range $1.7^{\mathrm{d}}-8.2^{\mathrm{d}}$. Obviously, his results are not conclusive. We have determined the period $P_{\text {mag }}=0.28313^{\mathrm{d}}$, which reproduces best the magnetic $B_{\mathrm{e}}$ variations of this star.

HD 36313. North (1984) presented the photometric period, $P_{\text {phot }}=0.58931^{\mathrm{d}}$. Borra (1981) found two probable magnetic periods, $P_{\text {mag }}=3.5^{\mathrm{d}}$ and $6.2^{\mathrm{d}}$. The above photometric period poorly reproduces variations of the collected $B_{\mathrm{e}}$ points.

HD 37776. The magnetic rotational phase curve has a very complicated form for $P_{\mathrm{mag}}=1.538675^{\mathrm{d}}$. The principal contribution to the phase curve comes from the second harmonic term, i.e. abs $\left(B_{2}\right) \gg$ abs $\left(B_{1}\right)$, see Eq. (6). Moreover, note that $B_{\mathrm{e}}$ measurements published in Ref. 24 appear also in Ref. 174. Therefore, we took into account only the latter paper for the construction of the magnetic rotational phase curve.

We arbitrarily adopted the rotational period two times larger but did not obtain a phase curve of acceptable quality. The origin of the actual complex phase curve remains unclear.

$H D$ 40312. Behavior of the $B_{\mathrm{e}}$ field of this star was studied in several research papers. We have constructed the average $B_{\mathrm{e}}(\phi)$ curve without observations both from Ref. 25 $\left(\mathrm{H}_{\alpha}\right.$ line) and Ref. 226 (photographic data), due to their low accuracy. Also measurements from Ref. 60 frequently duplicate those from Ref. 2, therefore we used Ref. 2 only $\left(\mathrm{H}_{\beta}\right.$ line). We found a new improved magnetic period $P_{\text {mag }}=3.61866^{\mathrm{d}}$ on the basis of data from Refs. 2 and 310.

High accuracy $B_{\mathrm{e}}$ measurements obtained from metal lines (Ref. 310) suggest that the phase curve deviates from a sine wave near phase $\phi=0.5$. We attempted to fit those data by a double wave.

HD 49606. The magnetic behavior of this star was investigated in Refs. 38 and 241 (phot. method), but with rather nonconclusive results. Meaningful $B_{\mathrm{e}}$ observations were published in Refs. 230 and 267 (hydrogen lines) and in Ref. 330 (metal lines).

HD 55719. The magnetic variability of this star was studied with the photographic method in Refs. 32 and 93. Results of those $B_{\mathrm{e}}$ observations were not conclusive, certainly due to the high projected rotational velocity $v \sin i \approx 77 \mathrm{~km} \mathrm{~s}^{-1}$. The number of new high accuracy $B_{\mathrm{e}}$ measurements in Ref. 256 is not sufficient to obtain credible conclusions regarding the magnetic variability of this star.

It is well known that the accuracy of Zeeman splitting measurements is related to the slope $\mathrm{d} I_{\lambda} / \mathrm{d} \lambda$ in spectral lines, and it decreases when lines are broadened e.g. by rotation. This is the case of HD 55719. Methods of $B_{\mathrm{e}}$ measurements and their disadvantages were presented in Sect. 5 of this paper. The accuracy of magnetic measurements was discussed in our previous paper, Bychkov et al. (2003).

$H D$ 62140. The best period $P_{\text {mag }}=4.28488^{\mathrm{d}}$. Behavior of $B_{\mathrm{e}}$ in this star was studied in Refs. 91, 62 and 310. The latter paper presents $B_{\mathrm{e}}$ observations of high accuracy. Available observational data show that the phase curve $B_{\mathrm{e}}(\phi)$ is best fitted by a double wave. 
HD $65339=53$ Cam. It is a very well investigated magnetic star. The longitudinal magnetic field $B_{\mathrm{e}}$ of this star was measured from its metal lines in Refs. 1, 9, 50, 105, 106, 110, $224,310,324$ and 327. Observations of $B_{\mathrm{e}}$ in hydrogen line wings were published in Refs. 25, 33, 74, 96, 190 and 327. Early $B_{\mathrm{e}}$ photographic measurements are of poor accuracy; however, they still yield a picture of $B_{\mathrm{e}}$ in this star, consistent with that derived from metal lines.

$H D$ 71866. The best magnetic period is $P_{\mathrm{mag}}=6.80024^{\mathrm{d}}$. The magnetic field was measured in Refs. 3, 6 and 196 (phot. method), and in Ref. 310 (LSD method). On average, the accuracy of LSD $B_{\mathrm{e}}$ observations is 6-8 times higher than photographic observations.

HD 112185. The magnetic behavior of this star was studied by many authors in Refs. 2, 25, 56, 123, 165, 182, 271, 310 and 334. The amplitude of $B_{\mathrm{e}}$ variations is small, therefore, they can only be investigated with methods of high accuracy. We did not use $B_{\mathrm{e}}$ observations from Refs. 25, 56, 123 and 165, due to their low accuracy. Most precise $B_{\mathrm{e}}$ measurements from metal lines were presented in Ref. 310 (LSD method).

$H D$ 112413. This is a well known and investigated magnetic star. The magnetic field $B_{\mathrm{e}}$ was investigated in Refs. 1, 25, 27, 33, 58, 77, 84, 99, 110, 123, 126, 211, 215, 217, 310, 318 and 327 . The average phase curves were derived only from $B_{\mathrm{e}}$ data of high accuracy.

$H D$ 118022. This is a well known and investigated magnetic star. Magnetic field measurements were presented in Refs. 1, 13, 22, 64, 76, 77, 81, 84, 310, 324 and 327. The average phase curves were derived only from $B_{\mathrm{e}}$ data of high accuracy.

HD 125248. Magnetic field determinations were published in Refs. 1, 2, 25, 88, 119, 184, 239, 256, 324 and 333. Measurements of $B_{\mathrm{e}}$ published in 1, 88 and 119 were not used in our paper due to low accuracy. Magnetic curves obtained from hydrogen or metal lines differ. The average phase curve for metal lines was obtained from $B_{\mathrm{e}}$ measurements published in Refs. 184, 239, 256, 324 and 333 (Fig. A.102). Note the very high accuracy of $B_{\mathrm{e}}$ values obtained by the LSD method in Ref. 333. The accuracy of their measurements is up to 10 times better than the accuracy of $B_{\mathrm{e}}$ obtained with other methods.

$H D$ 125823. Magnetic field $B_{\mathrm{e}}$ of this star was measured both in hydrogen and metal lines. The phase curves obtained from both series of $B_{\mathrm{e}}$ points differ substantially. We present separately the hydrogen and metallic $B_{\mathrm{e}}(\phi)$ phase curves, corresponding to measurements published in Refs. 37 and 176. There ia a large scatter of $B_{\text {e }}$ points clearly due to low accuracy of data. We need more high precision magnetic observations.

$H D$ 128898. Magnetic field $B_{\mathrm{e}}$ was measured in Refs. 2, $81,93,184$ and 256. Estimates in Ref. 93 were not used to construct $B_{\mathrm{e}}(\phi)$ in this paper because they were obtained by photographic methods of low accuracy. New high accuracy $B_{\mathrm{e}}$ observations are necessary to improve the magnetic rotational phase curve parameters.

HD 133029. Determination of the magnetic period of this star is still the essential problem. Measurements of $B_{\mathrm{e}}$ obtained from hydrogen lines strongly differ from those observed in metal lines. We assume here that the most credible period is $P_{\text {mag }}=2.105^{\mathrm{d}}$.
HD 137909. A magnetic star already extensively investigated. Its $B_{\mathrm{e}}$ field was measured by various techniques and the corresponding results were published in Refs. 1, 2, 17, 22, 25, $31,39,47,51,56,57,59,63,72,76,77,84,110,125,131,184$, $190,211,217,232,256,310,324$ and 327 . The average hydrogen phase curve $B_{\mathrm{e}}(\phi)$ presented in Fig. A.114 uses $B_{\mathrm{e}}$ values observed in $\mathrm{H}_{\beta}$ line wings, Refs. 2 and 76. Values of $B_{\mathrm{e}}$ presented in Refs. 25, 77 and 190 are of low accuracy. Moreover, some of them were measured in other lines of the Balmer series. Therefore we did not use these references.

There are also many published $B_{\mathrm{e}}$ points which were measured in metal lines. These observations were made with the most accurate LSD method, and also measurements were obtained from the Fe II 4520 line in Ref. 63. We present the corresponding phase curves in Figs. A.115 to A.118. Note that phase curves exhibit some differences in the above figures.

$H D$ 147010. The magnetic field $B_{\mathrm{e}}$ was measured in metal lines, see Refs. 41, 142, 184 and 256, and also in hydrogen lines - 168. We did not take into account photographic observations of low accuracy (Refs. 41 and 142) to construct the averaged magnetic rotational phase curve.

There is a systematic discrepancy between $B_{\mathrm{e}}$ measured in metal lines, and those observed in hydrogen lines. Therefore, we present here two distinctly differing $B_{\mathrm{e}}(\phi)$ phase curves for this star.

$H D$ 152107. A star with very interesting magnetic behavior. The magnetic field $B_{\mathrm{e}}$ has been studied by various methods. The principal magnetic period is $P_{\mathrm{mag}}=3.8^{\mathrm{d}}$, and there are variations on longer time scales. In the time interval JD 2442 400. - JD 2444 200, this star exhibited slow changes of its longitudinal magnetic field. Such an observation was claimed in Ref. 178, where measurements of the magnetic field were done in metal lines with the photographic method. However, magnetic field measurements published in Ref. 2 partly overlap this JD range, but they neither confirm nor deny the hyphothesis about $B_{\mathrm{e}}$ variations on long time scale. The latter $B_{\mathrm{e}}$ estimates were obtained from hydrogen lines with the photoelectric technique.

Therefore, the question of the magnetic behavior of this star remains open. Long time monitoring of HD 152107 with modern methods of high accuracy is necessary to solve this issue.

$H D$ 153882. The magnetic field $B_{\mathrm{e}}$ was measured by many authors in metal lines, Refs. 1, 6, 31, 72, 90, 184, 256 and 310. The average magnetic curve was obtained without $B_{\mathrm{e}}$ points from Refs. 1, 6 and 90, which were obtained by photographic methods of relatively low accuracy. The best magnetic period is $P_{\text {mag }}=6.00858^{\mathrm{d}}$.

$H D$ 170000. The magnetic field of this star was measured in hydrogen lines. The most accurate data are those in Ref. 60 (Fig. A.137, $\mathrm{H}_{\beta}$ line). Magnetic $B_{\mathrm{e}}$ measurements in Ref. 25 (Fig. A.138, $\mathrm{H}_{\alpha}$ line) are less accurate. Both phase curves have similar parameters. It is interesting to note that both phase curves exhibit a relative time shift by $0.5 \times P_{\mathrm{mag}}$, which is not obviously seen when comparing both figures. Moreover, if we try to construct a phase curve from both series of $B_{\mathrm{e}}$ points taken together, then any periodic variations of $B_{\mathrm{e}}$ vs. phase vanish. 
$H D$ 175362. Effective magnetic field $B_{\mathrm{e}}$ was measured by various methods. Estimates of $B_{\mathrm{e}}$ obtained from hydrogen lines and metal lines exhibit systematic differences. We have also rejected metal line $B_{\mathrm{e}}$ measurements of Ref. 52, due to their low accuracy (photographic method).

$H D$ 188041. The period of magnetic variability is $P_{\text {mag }}=223.826^{\mathrm{d}}$, according to Mikulasek et al. (2003). Measurements of $B_{\mathrm{e}}$ were performed by various methods, but only in metal lines. As it was shown in Ref. 232 results of magnetic field measurements strongly depend on the choice of element responsible for the development of lines in which $B_{\mathrm{e}}$ was measured. As a result, for example, $B_{\text {e }}$ points from Ref. 4 show strong variability with phase, whereas $B_{\mathrm{e}}$ observations from Refs. 1, 15, 16, 184 and 256, display variations with much smaller amplitude. Perhaps $B_{\mathrm{e}}$ measurements in hydrogen lines would yield a conclusive solution to the problem, because in the latter case results would not depend on the stratification of chemical elements over the stellar surface.

$H D$ 189849. Magnetic field $B_{\mathrm{e}}$ was measured in metal lines. One of the probable periods of magnetic variations is $P_{\text {mag }}=6.88449^{\mathrm{d}}$. Additional high precision $B_{\mathrm{e}}$ observations are necessary for the more precise determination of $P_{\text {mag }}$ and parameters of magnetic variability.

$H D$ 201601. The magnetic field $B_{\mathrm{e}}$ of this well known star was observed by various methods and results were presented in a large number of papers, cf. Refs. 1, 2, 46, 47, 48, 49, 105, 146, 184, 195, 232, 256, 268 and 327. The star shows "secular" $B_{\mathrm{e}}$ variability with the period $P_{\mathrm{sec}}=27027^{\mathrm{d}}-$ see Ref. 268 . Unfortunately, available observations do not cover the full pe$\operatorname{riod} P_{\mathrm{sec}}$. A precise description of the magnetic behavior of this star requires long-term monitoring of its $B_{\mathrm{e}}$ field. We also point out that possibly there exist systematic differences of $B_{\mathrm{e}}$ measured with different methods and by various authors.

$H D$ 215441. This star has a very strong magnetic field. Measurements of $B_{\mathrm{e}}$ in metal lines exhibit large scatter and did not yield the dependence of $B_{\mathrm{e}}$ on phase $\phi$. Measurements in hydrogen lines show periodic variability coincident with the rotational period $P_{\text {rot }}=9.4871^{\mathrm{d}}$. It is possible that there is also long period variability of $B_{\mathrm{e}}$, with the period $P_{\text {long }}=10180^{\mathrm{d}}$.

\subsection{Stars of other spectral classes}

The sample of non-Ap stars in the catalogue includes objects of various types. These are: supergiants of luminosity class I (HD 45348, HD 194093, and HD 207260), late-type dwarfs (HD 131156 and HD 201091), one close binary system with mass transfer (HD $174638=\beta$ Lyr), one pulsating variable star $($ HD $16582=\delta$ Cet $)$ and hot Ae/Be stars with emission lines (HD 37022 and HD 37041).

Spectral classification of some of catalogued stars can be ambiguous, of course. For example, the star HD 37041 mentioned above is of type $09.5 \mathrm{~V}$ pe and possibly should be included in the group of hot Bp stars.

We excluded HD 182989=RR Lyr from our catalogue, since this well-known pulsating star apparently does not have a strong global magnetic field and the periodic variability of its longitudinal component was not detected (see comments in this subsection).

The structure of the global magnetic fields in non-Ap stars can differ from those in Ap stars. It seems, however, that in the case of several stars collected in our catalog, the surface field integrated over the visible stellar disc is not equal to zero. There apparently exists a nonzero integrated dipole component, and its integrated projection on the line of sight varies with the rotational period. One can assume that such a weak field is the sum of small magnetic spots with strong fields, for instance.

$H D$ 37022. An Ae/Be star with the rotational period $P_{\text {rot }}=$ $15.422^{\mathrm{d}}$. Authors of Ref. 348 do not provide an estimate of the errors of their $B_{\mathrm{e}}$ determinations. Since the rotational period $P_{\text {rot }}$ is rather long, we have grouped individual $B_{\mathrm{e}}$ points in bins not larger than $0.1^{\mathrm{d}}$. Assuming that $B_{\mathrm{e}}$ does not change within such a small phase range, we were able to estimate the error of a single $B_{\mathrm{e}}$ measurement, $\sigma_{\mathrm{e}}=372 \mathrm{G}$.

$H D 174638=\beta$ Lyr. It is a close binary system with mass transfer between both components, with a nonzero magnetic field. Measurements $B_{\mathrm{e}}$ were performed mostly in metal lines of the blue optical region with the photographic method (Fig. A.142). Measurements of $B_{\mathrm{e}}$ within red silicon lines (Fig. A.143) reveal a lower level of effective magnetic field variations.

In principle this binary star should not exhibit a strong global magnetic field. It is possible that the above observations of $B_{\mathrm{e}}$ would not be confirmed by more accurate measurements in the future, similar to the case of $H D 182989$.

HD $182989=R R$ Lyr. It is a well known pulsating star. Photographic measurements showed variations of $B_{\mathrm{e}}$ in this star both with the pulsation period, $P_{\text {puls }}=0.566867^{\mathrm{d}}$, and the period of the Blazhko effect, $P_{\text {Blaz }}=40.8^{\mathrm{d}}$ (Romanov et al. 1985). However, the newest high precision measurements by Chadid et al. (2004) showed the absence of a global magnetic field in this star.

\section{Discussion: CP stars in our sample}

We have compiled average magnetic rotational phase curves $B_{\mathrm{e}}(\phi)$ for 136 stars. Most of them, 127 objects, are classified as chemically peculiar A and B type stars. Therefore we restricted most of the following discussion to this widely investigated group of main sequence stars.

The following subsections and figures present number distributions and statistical properties of various parameters: spectral types, rotational periods, fitting coefficients $B_{0}, B_{1}, B_{2}$ and the distribution of the parameter $r=B_{\mathrm{e}}(\max ) / B_{\mathrm{e}}(\min )$.

\subsection{Distribution of spectral types}

Figure 1 presents the distribution of stars in our catalog among various spectral types. Magnetic rotational phase curves $B_{\mathrm{e}}(\phi)$ are given mostly for stars of early spectral type (which are almost exclusively chemically peculiar stars). There are only 5 stars of spectral types cooler than F4.

Such a strong bias can be understood when considering that the field topology and intensity of CP stars make their global magnetic fields more easily detectable than in other types of 


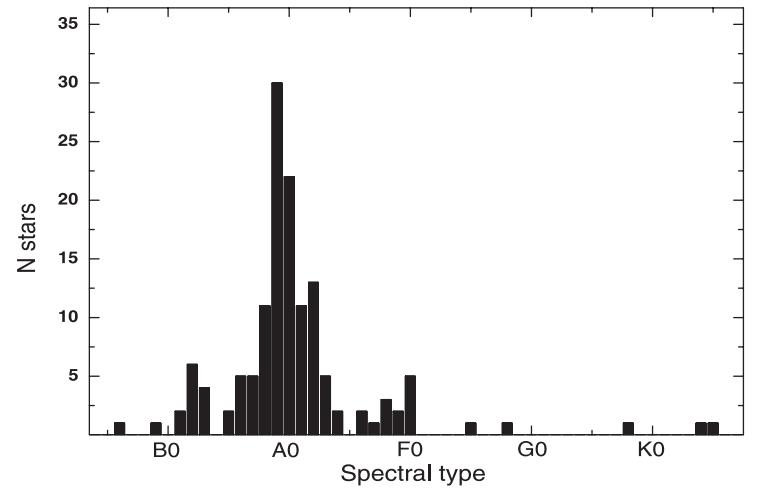

Fig. 1. Number distribution of stars with known $B_{\mathrm{e}}(\phi)$ phase curves vs. spectral type.

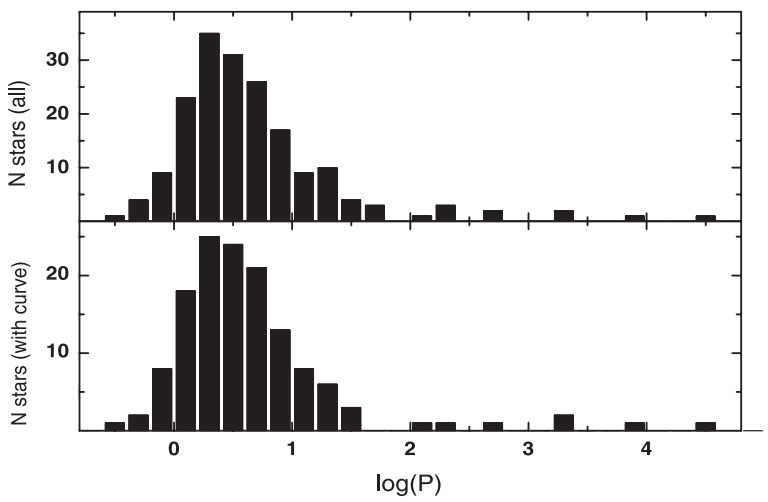

Fig. 2. Number distribution of stars in our catalog vs. decimal logarithm of period $P$, for all Ap stars with known periods (upper panel) and for stars with known phase curves (lower panel).

stars. Following the discovery of strong global magnetic fields in 78 Vir (HD 118022) by Babcock (1958), observers concentrated their efforts and interest mostly on stars of this class. Magnetic fields of stars belonging to other spectral classes were seldom measured, since such observations usually did not yield positive results.

Therefore, the present knowledge of the global magnetic fields across the whole spectral sequence on the Hertzsprung-Russell diagram is rather poor and selective.

\subsection{Distribution of periods}

Figure 2 presents the distribution of stars in our catalog vs. decimal logarithm of period $P$, separately for all $\mathrm{CP}$ stars with known periods (upper panel) and for stars whose period leads to consistent rotational phase curves (lower panel). The width of a single bin is 0.3 dex.

Approx. 60\% of all CP stars exhibit periods $P_{\text {mag }}$ between 1 and 5 days. The same is true for stars with known phase curves.

\subsection{Coefficients $B_{0}, B_{1}$, and $B_{2}$}

Figure 3 shows the number distribution vs. coefficient $B_{0}$ for the catalogued stars whose rotational phase curves $B_{\mathrm{e}}(\phi)$ were approximated by sine waves. Figure 3 does not include HD 215441 whose exceptionally strong $B_{\mathrm{e}}$ field exceeds the

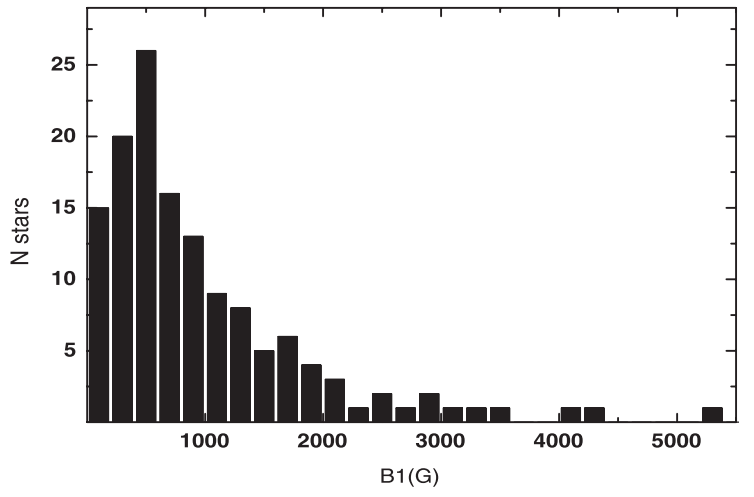

Fig. 3. Number distribution of catalogued stars vs. coefficient $B_{0}$ for those stars in which the phase curve $B_{\mathrm{e}}(\phi)$ was approximated by a sine wave.

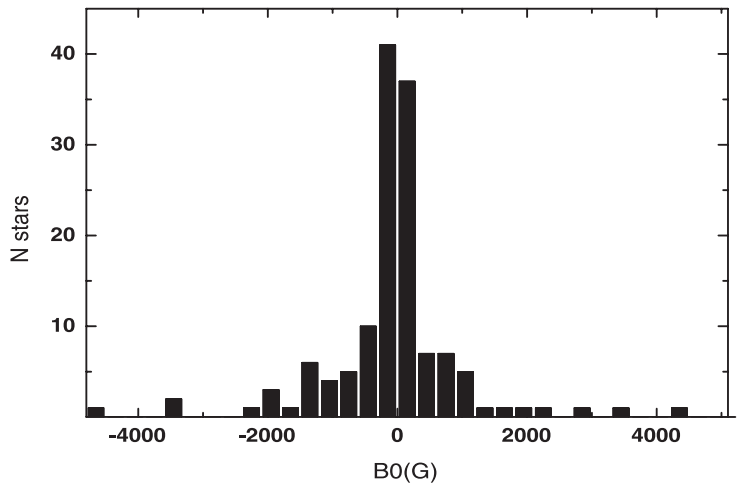

Fig. 4. Number distribution of catalogued stars vs. coefficient $B_{1}$ (half amplitude of $B_{\mathrm{e}}$ variability). This figure shows only those stars with a sine wave phase function.

scale of the figure. The average value of $B_{0}$ over all singlewave stars is $21+/-138 \mathrm{G}$, representing the width of the peak distribution.

Figure 4 displays an analogous number distribution vs. coefficient $B_{1}$ (half amplitude of $B_{\mathrm{e}}$ variability) for the catalogued stars with sine wave rotational phase curves $B_{\mathrm{e}}(\phi)$.

The number of stars with the lowest amplitude of $B_{\mathrm{e}}$ variations is underestimated. In fact, the accuracy of magnetic measurements is limited, and many stars with low magnetic variability were not detected as variable stars and therefore were not included in our catalog. This is the reason for the significant deficiency of stars with small half amplitude $B_{1}$ (see Fig. 4). We expect that future magnetic measurements of very high accuracy will improve the statistics at the lower values of the $B_{\mathrm{e}}$ distribution.

The amplitude of $B_{\mathrm{e}}$ variations depends, apart from Landè factor and field intensity, on the inclination of the rotational axis. A pole-on or a quasi pole-on star (that is, a star whose pole is along the line of sight, $i=0$ ) will never show any rotational modulation of $B_{\mathrm{e}}$.

Once the measurement accuracy is improved, the number of stars with small $B_{\mathrm{e}}$ variation is expected to raise.

Since the inclination angle of stars is randomly distributed, we have to conclude that $\mathrm{CP}$ stars favor configurations with small values of $\beta$. 


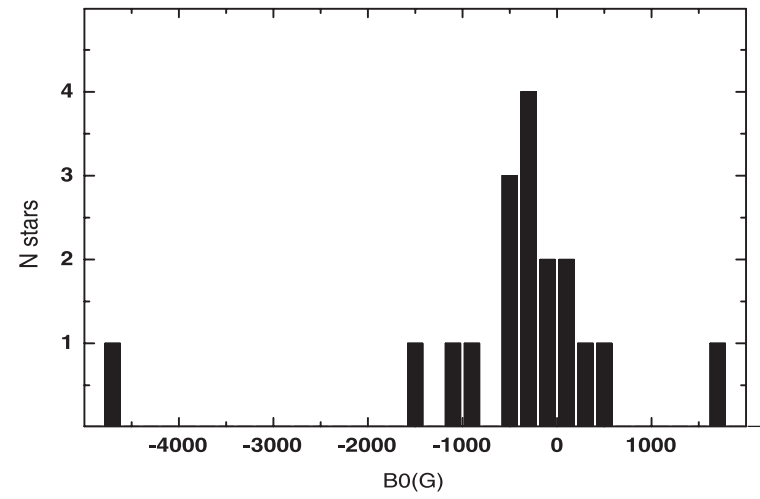

Fig. 5. Number distribution of stars vs. coeffcient $B_{0}$ for those stars which exhibit a magnetic rotational phase curve with a double wave.

Figure 5 shows the number distribution vs. coeffcient $B_{0}$ for those stars which exhibit magnetic rotational phase curves with double waves. The average value of $B_{0}$ is $-473+/-296 \mathrm{G}$. Again, the value of $296 \mathrm{G}$ represents the width of the peak distribution. The total number of stars in the figure is small (18 objects), therefore the statistics are poor. However, we conclude that stars with complex $B_{\mathrm{e}}$ phase curves (double waves) more frequently have negative values of $B_{\mathrm{e}}$.

\subsection{Parameter $r$}

We perform the following analysis according to the formalism by Preston (1967b). According to Eq. (4) one can determine the value of $i$

$i=\arctan \left[\frac{1-r}{1+r} \cot \beta\right]$.

The above equation determines the probability density of the occurence of a value $r$ at the fixed angle $\beta$

$p_{\beta}(r)=\frac{2(1-r) \tan \beta}{\left[(1-r)^{2}+(1+r)^{2} \tan ^{2} \beta\right]^{1 / 2}}$.

We assume that in our sample the rotational axis directions are uniformly distributed on the sphere. Consenquently, the occurence probability of the particular angle $\beta$ is proportional to the area of an infinitesimal ring of $\beta=$ const. We have determined probability of the occurence of $\beta$ in the range of $0^{\circ}-90^{\circ}$ with steps of $10^{\circ}$.

We have constructed relations $p_{\beta}(r)$ for eight fixed values of $\beta$ in the range $-1 \leq r \leq+1$ corresponding to the centers of bins defined in the above way. We then computed their arithmetic average with the weight proportional to the probability of the occurence of angle $\beta$.

Figure 6 presents the above averaged relation (solid line). This is the number distribution of stars in a uniformly distributed sample vs. the observed parameter $r$. The histogram represents the distribution of real stars in our catalogue. Figure 6 shows the substantial differences between the observed and theoretical number distributions of the parameter $r$. We interpret these differences either as a real nonuniformity in $\beta$ distribution, or as the result of poor statistics and the resulting random fluctuations.

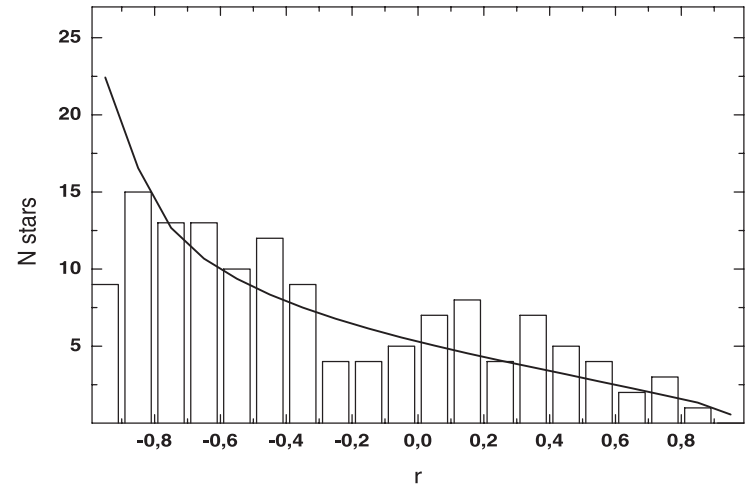

Fig. 6. Measured distribution of the parameter $r$ vs. theoretical distribution, the latter corresponding to the random distribution of $\beta$ and $i$ angles.

\section{Summary and conclusions}

We have compiled a catalog of magnetic rotational phase curves $B_{\mathrm{e}}(\phi)$ for 136 stars which exhibit periodic variations of the effective magnetic field $B_{\mathrm{e}}$. Most of the catalogued objects, 127 stars, are chemically peculiar A and B type stars. The catalog consists of figures which display individual $B_{\mathrm{e}}$ measurements, error bars and phase curves $B_{\mathrm{e}}(\phi)$ approximated either by a sine wave or by a double wave.

The catalog also presents a listing of the following parameters of magnetic phase curves: coefficients $B_{0}, B_{1}$, and $B_{2}$ of the harmonic expansion of $B_{\mathrm{e}}(\phi)$, the magnetic period $P_{\text {mag }}$ (in days) usually equal to the rotational period $P_{\text {rot }}$, Julian Day of the zero phase $T_{0}$ and the coefficient $r$ defined by Stibbs (1950). We estimated and listed errors for most of the above parameters of the magnetic $B_{\mathrm{e}}$ variations.

The above analysis is restricted to a very limited number of magnetic phase curve parameters. Detailed analysis of distributions and relations between the parameter $r$, the angle $\beta$ and the angle $i$ will be presented in a forthcoming paper.

We are aware that there are a number of magnetic stars with strong effective magnetic field $B_{\mathrm{e}}$ and very low or zero variation. Such stars were not included in our catalog, partly because it was impossible to determine the magnetic period $P_{\text {mag }}$ and phases of individual $B_{\mathrm{e}}$ measurements. We stress here that this fact introduces a particular bias in the catalogue, which neglects some groups of magnetic stars. The bias is responsible for the apparent deficiency of stars with the smallest amplitude of sine variations $B_{1}$, see Fig. 4 .

Our catalogue shows that approx. $14 \%$ of investigated magnetic stars exhibit complex magnetic rotational phase curves, which were approximated by a double wave (18 stars). The remaining 118 stars have simpler phase curves, approximated here by a simple sine wave. It is possible that some stars with simple sine phase curves will have a more complex shape of $B_{\mathrm{e}}(\phi)$ when more accurate magnetic data become available. Also we expect the discovery of new magnetic stars. Both reasons are likely to change the above proportion (14\%).

Acknowledgements. We are grateful to S. Messina, the referee, for his criticism and numerous suggestions which helped us to improve our paper. Our research is based on data compiled and 
posted in the SIMBAD, ADS, and CDS databases. We acknowledge support from the Polish Committee for Scientific Research grant No. 1 P03D 00126.

\section{References}

Adelman, S. J., \& Boyce, P. W. 1995, A\&AS, 114, 253

Adelman, S. J., \& Knox, J. R. Jr. 1994, A\&AS, 103, 1

Albrecht, R., Jenker, H., Weiss, W. W., \& Wood, H. J. 1977, A\&A, 58, 93

Angel, J. R. P., \& Landstreet, J. D. 1970, ApJ, 160, L147

Angel, J. R. P., Mcgraw, J. T., \& Stockman, H. S. 1973, ApJ, 184, L79

Babcock, H. W. 1954, ApJ, 120, 66

Babcock, H. W. 1958, ApJS, 3, 30, 141

Babcock, H. W. 1960, ApJ, 132, 521

Babcock, H. W., \& Burd, S. 1952, ApJ, 116, 8

Bagnulo, S., Landi degl'Innochenti, E., Landolfi, M., \& Leroy, J. L. 1995, A\&A, 295, 459

Blanco, C., Catalano, F. A., \& Strazzulla, G. 1978, A\&AS, 31, 205

Boesgaard, A. M. 1974, ApJ, 188, 567

Bohlender, D. A. 1989, A\&A, 220, 215

Bohlender, D. A. 1994, in Pulsation, Rotation and Mass Loss in Early Type Stars, ed. L. A. Balona, H. F. Henrichs, \& J.-L. Le Contel (Dordrecht, Holland: Luwer Academic Press), IAU Symp., 162, 155

Bohlender, D. A., \& Landstreet, J. D. 1990, ApJ, 358, L25

Bohlender, D. A., Brown, D. N., Landstreet, J. D., \& Thompson, I. B. 1987, ApJ, 323, 325

Bohlender, D. A., Landstreet, J. D., \& Thompson, I. B. 1993, A\&A, 269,355

Bonsack, W. K. 1976, ApJ, 209, 160

Bonsack, W. K. 1977, A\&A, 59, 195

Bonsack, W. K. 1981a, PASP, 93, 756

Bonsack, W. K. 1981b, A Peculiar Newsletter, 7, 7

Bonsack, W. K., \& Pilachowski, C. A. 1974, ApJ, 190, 327

Bonsack, W. K., Pilachowski, C. A., \& Wolff, S. C. 1974, ApJ, 187, 265

Borra, E. F. 1975, ApJ, 196, L109

Borra, E. F. 1981, ApJ, 249, L39

Borra, E. F. 1994, private communication

Borra, E. F., \& Landstreet, J. D. 1973, ApJ, 185, L139

Borra, E. F., \& Landstreet, J. D. 1975, PASP, 87, 961

Borra, E. F., \& Landstreet, J. D. 1977, ApJ, 212, 141

Borra, E. F., \& Landstreet, J. D. 1978, ApJ, 222, 226

Borra, E. F., \& Landstreet, J. D. 1979, ApJ, 228, 809

Borra, E. F., \& Landstreet, J. D. 1980, ApJS, 42, 421

Borra, E. F., \& Vaughan, A. H. 1977, ApJ, 216, 462

Borra, E. F., \& Vaughan, A. H. 1978, ApJ, 220, 924

Borra, E. F., Fletcher, J. M., \& Poeckert, R. 1981, ApJ, 247, 569

Borra, E. F., Landstreet, J. D., \& Thompson, I. 1983, ApJS, 53, 151

Borra, E. F., Edwards, G., \& Mayor, M. 1984, ApJ, 284, 211

Brown, D. N., \& Landstreet, J. D. 1981, ApJ, 246, 899

Brown, D. N., Landstreet, J. D., \& Thompson, I. A. 1981, 23rd Liège Coll., 195

Brown, D. N., Shore, S. N., \& Sonneborn, G. 1985, AJ, 90, 1354

Bychkov, V. D., \& Bychkova, L. V. 2002, personal communication

Bychkov, V. D., \& Shtol', V. G. 1997, Stellar Magnetic Fields, Proc. Int. Conf., 200

Bychkov, V. D., Fabrika, S. N., \& Shtol', V. G. 1991, Astron. Lett., 17,43

Bychkov, V. D., El'kin, V. G., \& Shtol', V. G. 1992, Stellar Magnetism, Int. Conf. Proc. (Russia: Nauka), 211
Bychkov, V. D., Kostynchuk, L. Yu., \& Shtol', V. G. 1997a, Stellar Magnetic Fields, Proc. Int. Conf., 110

Bychkov, V. D., Hubrig, S., \& Shtol', V. G. 1997b, Stellar Magnetic Fields, Proc. Int. Conf., 197

Bychkov, V. D., Shtol', V. G., Gerth, E., \& Kroll, R. 1997c, Stellar Magnetic Fields, Proc. Int. Conf., 204

Bychkov, V. D., Bychkova, L. V., \& Madej, J. 2003, A\&A, 407, 631

Catalano, F. A., \& Leone, F. 1991, A\&A, 244, 327

Catalano, F. A., \& Leone, F. 1993, A\&AS, 100, 319

Catalano, F. A., \& Leone, F. 1994, A\&AS, 108, 595

Catalano, F. A., \& Leone, F. 1996, A\&A, 311, 230

Catalano, F. A., \& Renson, P. 1998, A\&AS, 127, 421

Chadid, M., Wade, G. A., Shorlin, S. L. S., \& Landstreet, J. D. 2003, A\&A, 413, 1087

Chadid, M., Wade, G. A., Shorlin, S. L. S., \& Landstreet, J. D. 2004, A\&A, 413, 1087

Chuntonov, G. A. 2001, Bull. Spec. Astrophys. Obs., 51, 112

Conti, P. S. 1969, ApJ, 156, 661

Conti, P. S. 1970a, ApJ, 159, 723

Conti, P. S. 1970b, ApJ, 160, 1077

Donati, J.-F., Semel, M, Carter, B. D., Rees, D. E., \& Cameron, A. C. 1997, MNRAS, 291, 658

Donati, J.-F., Wade, G. A., Babel, J., et al. 2001, MNRAS, 326, 1265

El'kin, V. G. 1992, Stellar Magnetism, International Conference Proceedings (Russia: Nauka), 67

El'kin, V. G. 1998, Contributions of the Astronomical Observatory Skalnate Pleso, No. XXVII, 452

El'kin, V. G. 2000, private communication

El'kin, V. G., Romanyuk, I. I., \& Shtol', V. G. 1991, A Peculiar Newsletter, 21, 29

El'kin, V. G., Shtol', V. G., \& Romanyuk, I. I. 1997, Stellar Magnetic Fields, Proc. Int. Conf., 207

El'kin, V. G., Kudryavtsev, D. O., \& Romanyuk, I. I. 2002, Astron. Lett., 28, 195

Gerth, E. 1988, Magnetic Stars, Proc. Int. Conf., 78

Gerth, E. 1990b, Mitt. Karl-Schwarzschild Obs. Tautenburg, 125, 33

Gerth, E. 1990a, Astron. Nachr., 311, 41

Gerth, E. 1994, private communication

Gerth, E., Scholz, G., Glagolevskij, Yu. V., \& Romanyuk, I. I. 1991, Astron. Nachr., 312, 107

Gerth, E., Bychkov, V. D., Glagolevskij, Yu. V., \& Romanyuk, I. I. 1992, Stellar Magnetism, International Conference Proceedings (Russia: Nauka), 60

Glagolevskij, Yu. V., Kozlova, K. I., Kopylov, I. M., et al. 1977, Astron. Lett., 11, 500

Glagolevskij, Yu. V., Chuntonov, G. A., Naidenov, I. D., et al. 1979, Bull. Spec. Astrophys. Obs., 23, 5

Glagolevskij, Yu. V., \& Chunakova, N. M. 1985, Bull. Spec. Astrophys. Obs., 19, 37

Glagolevskij, Yu. V., \& Chuntonov, G. A. 1998, Bull. Spec. Astrophys. Obs., 45, 105

Glagolevskij, Yu.V., Bychkov, V. D., Iliev, I. K., Romanyuk, I. I., \& Chunakova, N. M. 1982, Astron. Lett., 8, 26

Glagolevskij, Yu.V., Bychkov, V. D., Romanyuk, I. I., \& Chunakova, N. M. 1985, Bull. Spec. Astrophys. Obs., 19, 28

Glagolevskij, Yu.V., Romanyuk, I. I., Naidenov, I. D., \& Shtol', V. G. 1989, Bull. Spec. Astrophys. Obs., 27, 34

Glagolevskij, Yu.V., El'kin, V. G., Romanyuk, I. I., \& Shtol', V. G. 1995, Astron. Lett., 21, 190

Gollnow, H. 1964, PASP, 74, 163

Gollnow, H. 1971, Observatory, 91, 37

Hatzes, A. P. 1991, MNRAS, 253, 89 
Hensberge, H. 1993, in Peculiar versus Normal Phenomena in AType and Related Stars, ed. M. M. Dworetsky, F. Castelli, \& R. Faraggiana, Proc. IAU Coll., 138, ASP Conf. Ser., 44, 547

Hensberge, H., \& De Loore, C. 1974, A\&A, 37, 367

Henrichs, H. F., de Jong, J. A., Donati, J.-F., et al. 2000, Magnetic fields of chemically peculiar and related stars, Proc. Int. Conf., 57 van den Heuvel, E. P. J. 1971, A\&A, 11, 461

Hildebrandt, G., Nikolow, A. S., Scholz, G., \& Schoneich, W. 1973, Astron. Nachr., 294, 175

Hildebrandt, G., Schoneich, W., Lange, D., Zelwanowa, E., \& Hempelmann, A. 1985, Publ. Astrophys. Obs. Potsdam, 32, 5

Huchra, J. 1972, ApJ, 174, 435

Johnstone, R. M., \& Penston, M. V. 1987, MNRAS, 227, 797

Jones, T. J., \& Wolff, S. C. 1974, PASP, 86, 67

Jones, T. J., Wolff, S. C., \& Bonsack, W. K. 1974, ApJ, 190, 579

Kemp, J. C., \& Wolstencroft, R. D. 1973, ApJ, 182, L43

Kerschbaum, F., \& Maitzen, H. M. 1991, A\&A, 246, 346

Kolev, D. Z. 1977, Astron. Lett., 3, 363

Kozlova, K. I., Glagolevskii, Yu. V., \& Klochkova, V. G. 1975, in Proc. Baku Conference Magnetic Ap Stars, ed. I. A. Aslanov, 75

Krause, F., \& Scholz, G. 1981, Comm. Spec. Astrophys. Obs., 32, 19

Kudryavtsev, D. O., Piskunov, N. E., Romanyuk, I. I., Chuntonov, G. A., \& Shtol', V. G. 2000, Magnetic fields of chemically peculiar and related stars, Proc. Int. Conf., 64

Kurtz, D. W., Sullivan, D. J., Martinez, P., \& Tripe, P. 1994, MNRAS, 243, 289

Kuvshinov, V. M. 1972, Astron. Tsirk., No. 682, 3

Kuvshinov, V. M., Hildebrandt, G., \& Schoneich, W. 1976, Astron. Nachr., 297, 182

Landstreet, J. D. 1982, ApJ, 258, 639

Landstreet, J. D. 1988, ApJ, 326, 967

Landstreet, J. D. 1990, ApJ, 352, L5

Landstreet, J. D. 2000, private communication

Landstreet, J. D., \& Borra, E. F. 1977, ApJ, 212, L43

Landstreet, J. D., \& Borra, E. F. 1978, ApJ, 224, L5

Landstreet, J. D., Borra, E. F., Angel, J. R. P., \& Illing, R. M. E. 1975, ApJ, 201, 624

Landstreet, J. D., Borra, E. F., \& Fontaine, G. 1979, MNRAS, 188, 609

Lanz, T., \& Mathys, G. 1991a, IBVS, 3655

Lanz, T., \& Mathys, G., 1991b, IBVS, 3655

Lanz, T., Bohlender, D. A., \& Landstreet, J. D. 1991, IBVS, 3678

Lebedev, V. S. 1986, Bull. Spec. Astrophys. Obs., 21, 21

Lebedev, V. S. 1990, Bull. Spec. Astrophys. Obs., 30, 83

Leckrone, D. S. 1974, ApJ, 190, 319

Leone, F., \& Catanzaro, G. 2001, A\&A, 365, 118

Leroy, J. L. 1995, A\&A, 114, 79

Lestrade, J.-F., Mutel, R. L., Preston, R. A., \& Phillips, R. B. 1985, Cool Stars, Stellar Systems, and the Sun, 254, 135

Madej, J. 1983, Acta Astron., 33, 1

Maitzen, H. M., Weiss, W. W., \& Wood, H. J. 1980, A\&A, 81, 323

Manfroid, J., \& Renson, P. 1980, IBVS, 1824

Mathews, J. M., \& Bohlender, D. A. 1991, A\&A, 243, 148

Mathys, G. 1991, A\&AS, 89, 121

Mathys, G. 1994, A\&AS, 108, 547

Mathys, G., \& Bohlender, D. A. 1991, A\&A, 243, 148

Mathys, G., \& Hubrig, S. 1997, A\&AS, 124, 475

Mathys, G., \& Lanz, T. 1992, A\&AS, 256, 169

Mathys, G., Manfroid, J., \& Renson, P. 1986, A\&AS, 63, 403

Mikulasek, Z., Glagolevskij, Yu. V., Romanyuk, I. I., Shtol', V. G., \& Bychkov, V. D. 1984, Conf. Magnetic Stars, 4, Riga, 13

Mikulasek, Z., Ziznovsky, J., Zverko, J., \& Polosukhina, N. 2003, Contr. Astron. Obs. Skalnate Pleso, 33, 29
Mohan, V. 1981, Ap\&SS, 76, 83

Monin, D. N., Fabrika, S. N., \& Valyavin, G. G. 2002, A\&A, 396, 131

Musielok, B., \& Madej, J. 1988, A\&A, 202, 143

Musielok, B., Lange, D., Schoneich, W., et al. 1980, Astron. Nachr., 301, 71

Neiner, C., Geers, V. C., Henrichs, H. F., et al. 2003, A\&A, 406, 1019

North, P. 1984, A\&AS, 55, 259

North, P. 1987, A\&AS, 69, 371

North, P., \& Adelman, S. J. 1995, A\&AS, 111, 41

Panchuk, V. E., Romanyuk, I. I., \& Kudriavtsev, D. O. 2000, Magnetic fields of chemically peculiar and related stars, Proc. Int. Conf., 75

Panov, K., \& Schoneich, W. 1976, Astron. Nachr., 297, 177

Pedersen, H. 1979, A\&AS, 35, 313

Pedersen, H., \& Thomsen, B. 1977, A\&AS, 30, 11

Pilachowski, C. A., Bonsack, W. K., \& Wolff, S. C. 1974, A\&A, 37, 275

Piskunov, N. E., \& Khohlova, V. L. 1983, Astron. Lett., 9, 665

Plachinda, S. I. 1990, Mitt. Astr. Obs. Crimea, 81, 112

Plachinda, S. I., \& Tarasova, T. N. 1999, ApJ, 514, 402

Plachinda, S. I., \& Tarasova, T. N. 2000a, Magnetic fields of chemically peculiar and related stars, Proc. Int. Conf., 73

Plachinda, S. I., \& Tarasova, T. N. 2000b, ApJ, 533, 1016

Plachinda, S. I., Johns-Krull, C. M., \& Tarasova, T. N. 2001 (Odessa Astronomical Publications), 14, 219

Plachinda, S. I., Jakuschechkin, A. V., \& Sergeev, S. G. 1993, Mitt. Astr. Obs. Crimea, 87, 91

Preston, G. W. 1967a, ApJ, 150, 547

Preston, G. W. 1967b, ApJ, 150, 871

Preston, G. W. 1969a, ApJ, 157, 247

Preston, G. W. 1969b, ApJ, 158, 251

Preston, G. W. 1970, ApJ, 160, 1059

Preston, G. W. 1971, ApJ, 164, 309

Preston, G. W. 1972, ApJ, 175, 465

Preston, G. W., \& Stępień, K. 1968a, ApJ, 151, 577

Preston, G. W., \& Stȩpień, K. 1968b, ApJ, 154, 971

Preston, G. W., \& Wolff, S. C. 1970, ApJ, 160, 1071

Preston, G. W., Stępień, K., \& Wolff, S. C. 1969, ApJ, 156, 653

Pyper, D. M., \& Adelman, S. J. 1985, A\&AS, 59, 369

Rakosch, K. D., \& Fiedler, W. 1978, A\&AS, 31, 83

Rakos, K. D., Schermann, A., Weiss, W. W., \& Wood, H. J. 1977, A\&A, 56, 453

Renson, P. 1972, A\&A, 18, 159

Renson, P. 1984, A\&A, 139, 131

Renson, P., \& Catalano, F. A. 2001, A\&A, 378, 113

Rice, J. B., \& Wehlau, W. H. 1994, A\&A, 291, 825

Robinson, R. D. 1980, ApJ, 239, 961

Robinson, R. D., Worden, S. P., \& Harvey, J. W. 1980, ApJ, 236, L155

Romanov, Yu. S., Udovichenko, S. N., \& Frolov, M. S. 1985, Astron. Lett., 5, 378

Romanov, Yu. S., Udovichenko, S. N., \& Frolov, M. S. 1988, Magnetic Stars (USSR: Nauka), 51

Rudiger, G., \& Scholz, G. 1988, Astron. Nachr., 309, 181

Rudy, R. J., \& Kemp, C. J. 1978, MNRAS, 183, 595

Rustamov, Yu. S., \& Khotnyanskij, A. N. 1980, Astron. Lett., 6, 364

Ryabchikova, T. A., Davydova, E. S., \& Kolev, D. Z. 1988, Magnetic Stars (USSR: Nauka), 40

Saar, S. H. 1988, ApJ, 324, 441

Sargent, W. L. W., Sargent, A. I., \& Strittmatter, P. A. 1967, ApJ, 147, 1185

Scholz, G. 1971, Astron. Nachr., 292, 281

Scholz, G. 1975, Astron. Nachr., 296, 31

Scholz, G. 1978, Astron. Nachr., 299, 81

Scholz, G. 1979, Astron. Nachr., 300, 213 
Scholz, G. 1983, Astrophys. Space Sci., 94, 159

Scholz, G., \& Gerth, E. 1980, Astron. Nachr., 301, 211

Schoneich, W., Hildebrandt, G., \& Furtig, W. 1976, Astron. Nachr., No. 297,39

Severny, A. 1970, ApJ, 159, L73

Shore, S. N., \& Brown, D. N. 1990, ApJ, 365, 665

Shore, S. N., Brown, D. N., Sonneborn, G., Landstreet, J. D., \& Bohlender, D. A. 1990, ApJ, 348, 242

Shorlin, S. L. S., Wade, G. A., Donati, J.-F., et al. 2002, A\&A, 392, 637

Shtol', V. G., Bychkov, V. D., Vikuliev, N. A., et al. 1985, Bull. Spec. Astrophys. Obs., 19, 66

Shtol', V. G., Polyakov, V. I., Konojko, A. N., \& Vikulev, N. A. 1992, Stellar Magnetism, Inter. Conf. Proc. (Russia: Nauka), 190

Skulskij, M. Yu. 1982, Astron. Lett., 8, 238

Skulskij, M. Yu. 1985, Astron. Lett., 11, 51

Skulskij, M. Yu. 1990, Mitteilungen des Karl-SchwarzschildObservatoriums Tautenburg, 125, 146

Skulskij, M. Yu., \& Plachinda, S. I. 1993, Astron. Lett., 19, 517

Skulskij, M. Yu., Najdenov, I. D., Romanyuk, I. I., \& Bychkov, V. D. 1992a, Stellar Magnetism, Proc. Int. Conf. (Russia: Nauka), 204

Skulskij, M.Yu., Plachinda, S. I., \& Malkov, Yu. F. 1992b, Stellar Magnetism, Proc. Int. Conf. (Russia: Nauka), 216

Slovak, M. H. 1982, ApJ, 262, 282

Smirnov, D. A., Fabrika, S. N., Lamzin, S. A., \& Valyavin, G. G. 2003a, A\&A, 401, 1057

Smirnov, D. A., Lamzin, S. A., \& Fabrika, S. N. 2003b, Astron. Lett., 29,258

Stahl, O., Kaufer, A., Rivinius, Th., et al. 1996, A\&A, 312, 539

Steenbeck, M., \& Krause, F. 1968, Astron. Nachr., 291, 49

Stępień, K., \& Czechowski, W. 1993, A\&A, 268, 187

Steinitz, R., \& Pyper, D. M. 1970, IBVS, 413

Stibbs, D. W. 1950, MNRAS, 110, 395

Tarasova, T. N., Plachinda, S. I., \& Rumyantsev, V. V. 2001, Astron. Lett., 78, 550

Thompson, I. B. 1983, MNRAS, 205, 43

Thompson, I. B., \& Landstreet, J. D. 1985, ApJ, 289, L9

Thompson, I. B., Brown, D. N., \& Landstreet, J. D. 1987, ApJS, 64, 219

van Genderen, A. M. 1964, IBVS, 76

van Genderen, A. M. 1971, A\&A, 14, 48
Verdugo, E., Talavera, A., Gomez de Castro, A. I., \& Henrichs, H. 2002, A Massive Star Odyssey, From Main Sequence to Supernova, Proc. IAU Symp., 212, in press

Vogt, S. S., Tull, R. G., \& Kelton, P. W. 1980, ApJ, 236, 308

Wade, G. A., El'kin, V. G., Landstreet, J. D., et al. 1996, A\&A, 313, 209

Wade, G. A., Donati, J.-F., Landstreet, J. D., \& Shorlin, S. L. S. 2000a, MNRAS, 313, 851

Wade, G. A., Kudryavtsev, D., Romanyuk, I. I., Landstreet, J. D., \& Mathys, G. 2000b, A\&A, 355, 1080

Wade, G. A., Donati, J.-F., Landstreet, J. D., \& Shorlin, S. L. S. 2000c, MNRAS, 313, 823

Wade, G. A., Chadid, M., Shorlin, S. L. S., Bagnulo, S., \& Weiss, W. W. 2002, A\&A, 392, L17

Weiss, W. W. 1986, A\&A, 160, 243

Weiss, W. W., \& Wood, H. J. 1975, A\&A, 41, 165

Weiss, W. W., Jenkner, H., \& Wood, H. J. 1978, A\&A, 63, 247

Weiss, W. W., Malanushenko, V. P., \& Shakhovskoy, N. M. 1990, Mitt. Astr. Obs. Crimea, 82, 69

Willson, R. F., Lang, K. R., \& Foster, P. 1988, A\&A, 199, 255

Wolff, S. C. 1969a, ApJ, 157, 253

Wolff, S. C. 1969 b, ApJ, 158, 1231

Wolff, S. C. 1973, ApJ, 186, 951

Wolff, S. C. 1975, ApJ, 202, 127

Wolff, S. C., \& Bonsack, W. K. 1972, ApJ, 176, 425

Wolff, S. C., \& Morrison, N. D. 1974, PASP, 86, 935

Wolff, S. C., \& Morrison, N. D. 1975, PASP, 87, 231

Wolff, S. C., \& Preston, G. W. 1978, PASP, 90, 406

Wolff, S. C., \& Wolff, R. J. 1970, ApJ, 160, 1049

Wolff, S. C., \& Wolff, R. J. 1972, ApJ, 176, 433

Wolstencroft, R. D., Smith, R. J., \& Clarce, D. 1981, MNRAS, 195, 39

Wood, H. J., \& Campusano, L. B. 1975, A\&A, 45, 303

Ziznovsky, J., \& Romanyuk, I. I. 1990, Bull. Astron. Institutes Czechoslovakia, 41, 118

Zverko, J. 1987, Contributions of the Astronomical Observatory Skalnate Pleso, 16, 7

Zverko, J., Bychkov, V. D., Ziznovsky, J., \& Hric, L. 1989, Contributions of the Astronomical Observatory Skalnate Pleso, 18,71 


\section{Online Material}


V. D. Bychkov et al.: A catalog of stellar magnetic rotational phase curves, Online Material p 2

Table 1. Single wave $B_{\mathrm{e}}$ phase curve parameters for all catalogued stars. Julian Day of zero phase is JD $=2400000 .+T_{0}$. An asterisk attached to the HD number indicates that the phase curve of this star has to be fitted by a double wave, and the star is also listed in Table 3. Parameters $B_{0}, \sigma_{B_{0}}, B_{1}, \sigma_{B_{1}}$ are given in $\mathrm{G}$, and $T_{0}, \sigma_{T_{0}}$ are in days.

\begin{tabular}{|c|c|c|c|c|c|c|c|c|}
\hline HD & Sp. type & $B_{0}$ & $\sigma_{B_{0}}$ & $\overline{B_{1}}$ & $\sigma_{B_{1}}$ & $P$ (days) & $\bar{T} T_{0}$ & $\overline{\sigma_{T_{0}}}$ \\
\hline 2453 & A1 & -680 & 17 & 272 & 24 & 520.5 & 33363.490 & 5.554 \\
\hline 3360 & $\mathrm{~B} 2 \mathrm{~V}$ & -18 & 3 & 27 & 7 & 5.370447 & 52266.338 & 0.124 \\
\hline 3980 & A7p & 70 & 38 & 1740 & 91 & 3.9516 & 40929.344 & 0.021 \\
\hline 4778 & A1p & 166 & 103 & 1257 & 121 & 2.5616 & 47106.935 & 0.049 \\
\hline 5737 & B6p & 103 & 25 & 391 & 41 & 21.654 & 44691.696 & 0.308 \\
\hline 8441 & A2 & -166 & 50 & 350 & 81 & 1.80889 & 33570.246 & .063 \\
\hline $9996 *$ & B9p & -252 & 34 & 855 & 59 & 7692. & 33638.387 & 50.77 \\
\hline 10783 & A2 & 829 & 129 & 670 & 176 & 4.14628 & 33486.589 & 0.153 \\
\hline 11503 & A1p & -238 & 31 & 672 & 43 & 1.6093 & 43001.390 & 0.016 \\
\hline 12288 & $\mathrm{~A} 2 \mathrm{p}$ & -1508 & 57 & 1070 & 71 & 34.79 & 49895.485 & 0.439 \\
\hline 12447 & A0p & -49 & 31 & 473 & 49 & 1.49070 & 43495.498 & 0.021 \\
\hline 12767 & B9.5p & 29 & 43 & 277 & 47 & 1.892 & 43497.765 & 0.095 \\
\hline 14437 & B9p & 1918 & 61 & 726 & 60 & 28.87 & 44617.847 & 0.416 \\
\hline 15144 & A6Vp & -792 & 41 & 124 & 62 & 15.88 & 32461.798 & 1.440 \\
\hline 16582 & B2IV & -387 & 245 & 1231 & 355 & 1.40744 & 42350.922 & 0.066 \\
\hline \multirow[t]{2}{*}{18296} & B9p & 97 & 66 & 197 & 97 & 2.8842 & 33248.996 & 0.269 \\
\hline & & 30 & 40 & 173 & 54 & 2.8842 & 33249.445 & 0.177 \\
\hline 19832 & B9p & 42 & 71 & 378 & 87 & 0.72 & 37667.527 & 0.036 \\
\hline 21699 & B8IIIp & -84 & 74 & 822 & 95 & 2.4 & 44859.033 & 0.055 \\
\hline 22470 & B9p & 109 & 77 & 1119 & 123 & 0.6785 & 41947.107 & 0.010 \\
\hline 24155 & B9p & 561 & 145 & 999 & 188 & 2.53465 & 43841.124 & 0.089 \\
\hline 24712 & A9p & 705 & 31 & 471 & 43 & 12.4 & 40570.628 & 0.192 \\
\hline 25267 & $\mathrm{~B} 9+\mathrm{B} 9.5 \mathrm{~V}$ & -181 & 35 & 178 & 46 & 5.95 & 43498.005 & 0.284 \\
\hline 25354 & A2 & -221 & 316 & 290 & 629 & 3.90072 & 35788.888 & 0.629 \\
\hline 25823 & B9p & 366 & 118 & 570 & 153 & 4.65 & 34247.731 & 0.252 \\
\hline 27309 & $\mathrm{~A} 0 \mathrm{p}$ & 1479 & 166 & 1363 & 264 & 1.10 & 42768.266 & 0.028 \\
\hline 27962 & A3V & 682 & 61 & 623 & 92 & 2.13 & 41319.588 & 0.043 \\
\hline 28843 & $\mathrm{~B} 5-\mathrm{BC}$ & -33 & 112 & 479 & 159 & 1.37 & 43441.390 & 0.081 \\
\hline 30466 & A0p & 978 & 123 & 981 & 133 & 1.3900 & 35780.741 & 0.045 \\
\hline $32633^{*}$ & B9p & -1083 & 40 & 3076 & 45 & 6.43 & 35782.995 & 0.030 \\
\hline 34452 & $\mathrm{~A} 0 \mathrm{p}$ & 342 & 78 & 745 & 125 & 2.4 & 37294.864 & 0.056 \\
\hline 35298 & B6 & 661 & 207 & 3520 & 392 & 1.85 & 44597.448 & 0.025 \\
\hline 35456 & B6 & 161 & 95 & 1874 & 157 & 0.28313 & 46448.872 & 0.003 \\
\hline 35502 & B5 & -1853 & 179 & 1732 & 326 & 1.70 & 44591.152 & 0.033 \\
\hline 35912 & $\mathrm{~B} 2 \mathrm{~V}$ & -283 & 113 & 1448 & 174 & 0.89786 & 39571.019 & 0.015 \\
\hline 36313 & B8p & 87 & 225 & 1261 & 274 & 0.58931 & 44590.126 & 0.022 \\
\hline 36485 & $\mathrm{~B} 2 \mathrm{p}$ & -3359 & 135 & 1499 & 349 & 2.0129 & 45290.138 & 0.037 \\
\hline 36526 & B8 & 1630 & 196 & 2409 & 287 & 1.5405 & 43507.101 & 0.028 \\
\hline 36629 & B3 & -362 & 249 & 1485 & 324 & 5.01255 & 39168.313 & 0.167 \\
\hline 36668 & B7 & 34 & 247 & 1285 & 328 & 2.1211 & 44589.414 & 0.127 \\
\hline 37017 & $\mathrm{~B} 1.5 \mathrm{~V}$ & -1265 & 49 & 984 & 72 & 0.901175 & 43440.322 & 0.010 \\
\hline 37022 & O6pe & 72 & 68 & 333 & 138 & 15.422 & 50308.228 & 1.085 \\
\hline 37041 & O9.5Vpe & 970 & 146 & 1165 & 249 & 3.14264 & 41714.286 & 0.075 \\
\hline 37058 & B3p & 1016 & 144 & 1863 & 255 & 1.022 & 45299.515 & 0.014 \\
\hline 37140 & B8 & -122 & 273 & 681 & 483 & 2.7088 & 44594.642 & 0.250 \\
\hline 37151 & B8p & -38 & 166 & 501 & 267 & 5.6732 & 44323.826 & 0.502 \\
\hline
\end{tabular}


Table 1. continued.

\begin{tabular}{|c|c|c|c|c|c|c|c|c|}
\hline $\mathrm{HD}$ & Sp. type & $B_{0}$ & $\sigma_{B_{0}}$ & $B_{1}$ & $\sigma_{B_{1}}$ & $P$ (days) & $T_{0}$ & $\sigma_{T_{0}}$ \\
\hline 37210 & B8p & -220 & 271 & 643 & 466 & 11.0494 & 44589.291 & 1.176 \\
\hline 37479 & $\mathrm{~B} 2 \mathrm{Vp}$ & 671 & 83 & 2283 & 120 & 1.190811 & 43441.178 & 0.010 \\
\hline 37642 & B9p & -227 & 346 & 2943 & 805 & 1.07977 & 44589.817 & 0.064 \\
\hline $37776^{*}$ & B3 & -290 & 55 & 275 & 67 & 1.538675 & 45724.124 & 0.020 \\
\hline $40312 *$ & A0p & 68 & 9 & 288 & 14 & 3.61866 & 42764.658 & 0.026 \\
\hline 45348 & FOIb & 293 & 21 & 333 & 32 & 6.90 & 41001.970 & 0.097 \\
\hline 49333 & B7IIIn & 113 & 106 & 1035 & 189 & 2.18010 & 47310.482 & 0.044 \\
\hline 49606 & B7III & -218 & 83 & 413 & 108 & 1.10503 & 43819.373 & 0.046 \\
\hline 49976 & Alp & -163 & 127 & 1766 & 174 & 2.97668 & 35758.274 & 0.053 \\
\hline 51418 & $\mathrm{~A} 0$ & 195 & 117 & 476 & 171 & 5.631 & 41581.158 & 0.362 \\
\hline 54118 & $\mathrm{~A} 0 \mathrm{p}$ & -21 & 65 & 1364 & 87 & 3.27533 & 46829.614 & 0.037 \\
\hline 55719 & $A 3 p$ & 1044 & 141 & 876 & 219 & 1.00335 & 41642.046 & 0.033 \\
\hline 58260 & B3 & 2283 & 95 & 219 & 165 & 1.657 & 43503.502 & 0.176 \\
\hline $62140 *$ & A8p & 22 & 69 & 1678 & 89 & 4.28488 & 41254.991 & 0.037 \\
\hline $62140 *$ & & -52 & 14 & 1606 & 21 & 4.28488 & 41255.021 & 0.008 \\
\hline 64740 & $\mathrm{~B} 1.5 \mathrm{Vp}$ & -229 & 24 & 678 & 33 & 1.33026 & 43498.438 & 0.011 \\
\hline \multirow[t]{2}{*}{$65339 *$} & $A 3 p$ & -345 & 26 & 4389 & 37 & 8.0267 & 35854.246 & 0.010 \\
\hline & & -74 & 77 & 4151 & 119 & 8.0267 & 35854.083 & 0.033 \\
\hline 71866 & Alp & 222 & 27 & 2010 & 34 & 6.80024 & 32955.956 & 0.022 \\
\hline 72968 & $A 2 p$ & 40 & 89 & 549 & 148 & 4.66458 & 33253.586 & 0.210 \\
\hline 73340 & B9 & -1391 & 100 & 742 & 127 & 2.66753 & 43171.646 & 0.102 \\
\hline 74521 & A1p & 651 & 42 & 135 & 55 & 7.76851 & 33606.860 & 0.654 \\
\hline 77350 & B9p & -177 & 63 & 472 & 109 & 4.024 & 35879.465 & 0.130 \\
\hline 78316 & B8IIIp & -25 & 48 & 175 & 62 & 5.0035 & 34012.184 & 0.344 \\
\hline 79158 & B9IIIp & -33 & 41 & 881 & 55 & 3.8345 & 43647.801 & 0.040 \\
\hline 83368 & A8p & -9 & 51 & 704 & 81 & 2.851962 & 46218.974 & 0.044 \\
\hline 89822 & $\mathrm{~A} 0 \mathrm{p}$ & 52 & 37 & 100 & 56 & 7.5586 & 35566.464 & 0.712 \\
\hline 90044 & B9p & -4 & 106 & 1146 & 183 & 4.379 & 46893.138 & 0.095 \\
\hline 90569 & A0p & 101 & 197 & 318 & 372 & 1.445 & 32988.947 & 0.160 \\
\hline 92664 & B9p & -705 & 29 & 508 & 45 & 1.67315 & 43946.465 & 0.020 \\
\hline 96446 & $\mathrm{~B} 2$ & -1238 & 75 & 597 & 110 & 0.85521 & 43500.247 & 0.021 \\
\hline 96707 & F0p & 135 & 65 & 527 & 89 & 2.49183 & 40331.707 & 0.069 \\
\hline 98088 & A8IVp & -128 & 138 & 997 & 185 & 5.81714 & 34726.490 & 0.193 \\
\hline 103192 & B9IIIp & -228 & 68 & 93 & 112 & 2.344 & 43730.962 & 0.274 \\
\hline 108662 & A0p & -361 & 38 & 480 & 51 & 5.0805 & 32978.132 & 0.100 \\
\hline 108945 & $\mathrm{~A} 2 \mathrm{Vp}$ & -317 & 100 & 737 & 125 & 1.92442 & 42490.168 & 0.054 \\
\hline 110379 & F0V & 61 & 22 & 306 & 31 & 12.92696 & 34410.489 & 0.197 \\
\hline 111133 & A1p & -915 & 65 & 531 & 88 & 16.3078 & 35877.680 & 0.396 \\
\hline \multirow[t]{2}{*}{112185} & $\mathrm{~A} 1$ & 25 & 10 & 76 & 16 & 5.0887 & 41792.085 & 0.198 \\
\hline & & 36 & 8 & 67 & 10 & 5.0887 & 41792.085 & 0.158 \\
\hline 112381 & A0 & -3402 & 119 & 430 & 188 & 2.84 & 46831.344 & 0.204 \\
\hline \multirow[t]{3}{*}{112413} & A0 & -156 & 12 & 686 & 16 & 5.46939 & 50492.670 & 0.023 \\
\hline & & -180 & 20 & 1020 & 31 & 5.46939 & 41790.936 & 0.025 \\
\hline & & -222 & 27 & 1121 & 38 & 5.46939 & 41790.938 & 0.032 \\
\hline 116458 & A0p & -1954 & 39 & 361 & 51 & 2.3596 & 41010.295 & 0.055 \\
\hline \multirow[t]{2}{*}{118022} & $\mathrm{~A} 2 \mathrm{p}$ & -559 & 9 & 421 & 14 & 3.722084 & 50492.232 & 0.019 \\
\hline & & -507 & 53 & 369 & 78 & 3.722084 & 42493.460 & 0.124 \\
\hline 119213 & $\mathrm{~A} 2 \mathrm{Vp}$ & 630 & 79 & 770 & 127 & 2.44997 & 43139.882 & 0.064 \\
\hline 119419 & A0p & -614 & 77 & 2412 & 112 & 2.6006 & 46881.845 & 0.017 \\
\hline 122532 & B9p & -18 & 44 & 894 & 71 & 3.68137 & 46912.511 & 0.044 \\
\hline 124224 & B9Vp & 176 & 38 & 541 & 50 & 0.520675 & 42850.109 & 0.009 \\
\hline \multirow[t]{2}{*}{125248} & A1p & 56 & 108 & 2654 & 153 & 9.2954 & 34461.181 & 0.082 \\
\hline & & 73 & 44 & 2176 & 59 & 9.2954 & 34461.314 & 0.043 \\
\hline \multirow[t]{2}{*}{125823} & B7IIIpv & 35 & 83 & 516 & 113 & 8.8171 & 43724.005 & 0.361 \\
\hline & & 13 & 31 & 406 & 44 & 8.8171 & 43724.711 & 0.188 \\
\hline 126515 & $\mathrm{~A} 2 \mathrm{p}$ & -268 & 61 & 2176 & 81 & 129.95 & 37004.582 & 0.874 \\
\hline 128898 & A0p & -244 & 54 & 166 & 77 & 4.4794 & 43743.166 & 0.403 \\
\hline 131156 & $\mathrm{G} 8 \mathrm{~V}+\mathrm{K} 4 \mathrm{Ve}$ & 13 & 2 & 14 & 3 & 6.1455 & 44030.792 & 0.178 \\
\hline
\end{tabular}


V. D. Bychkov et al.: A catalog of stellar magnetic rotational phase curves, Online Material p 4

Table 1. continued.

\begin{tabular}{|c|c|c|c|c|c|c|c|c|}
\hline $\mathrm{HD}$ & Sp. type & $B_{0}$ & $\sigma_{B_{0}}$ & $B_{1}$ & $\sigma_{B_{1}}$ & $P$ (days) & $T_{0}$ & $\sigma_{T_{0}}$ \\
\hline \multirow{2}{*}{133029} & B9p & 3516 & 77 & 1455 & 205 & 2.105 & 33109.683 & 0.022 \\
\hline & & 2184 & 53 & 243 & 79 & $2 . .105$ & 33109.672 & 0.135 \\
\hline 133652 & $\mathrm{~A} 0 \mathrm{p}$ & -347 & 65 & 1995 & 152 & 2.3040 & 48344.814 & 0.014 \\
\hline 133880 & B9p & -275 & 50 & 3324 & 76 & 0.877485 & 42120.905 & 0.003 \\
\hline 134793 & $\mathrm{~A} 4$ & -114 & 110 & 532 & 177 & 2.7800 & 35941.897 & 0.145 \\
\hline 137509 & B8p & 178 & 107 & 973 & 145 & 4.4912 & 48342.302 & 0.112 \\
\hline \multirow[t]{5}{*}{137909} & A9p & 143 & 13 & 528 & 17 & 18.4868 & 34210.963 & 0.104 \\
\hline & & 102 & 5 & 671 & 8 & 18.4868 & 43309.186 & 0.033 \\
\hline & & -51 & 4 & 1242 & 7 & 18.4868 & 43309.746 & 0.012 \\
\hline & & -46 & 36 & 753 & 54 & 18.4868 & 43310.222 & 0.178 \\
\hline & & 108 & 21 & 524 & 32 & 18.4868 & 43310.221 & 0.151 \\
\hline 137949 & F0p & 1121 & 63 & 637 & 88 & 11.13313 & 36007.100 & 0.217 \\
\hline 140160 & $\mathrm{~A} 0 \mathrm{p}$ & -456 & 508 & 901 & 753 & 1.59584 & 40763.340 & 0.181 \\
\hline 142301 & B8IIIp & -1134 & 93 & 2848 & 157 & 1.45955 & 42593.469 & 0.011 \\
\hline 142990 & B6IV & -498 & 72 & 1272 & 84 & 0.9791 & 43560.066 & 0.012 \\
\hline 143473 & B9p & 4434 & 161 & 1378 & 331 & 2.84263 & 47270.549 & 0.066 \\
\hline 144334 & B8 & -523 & 73 & 778 & 103 & 1.49497 & 43704.004 & 0.030 \\
\hline \multirow[t]{2}{*}{147010} & B9p & -4717 & 97 & 1200 & 139 & 3.920676 & 45041.014 & 0.071 \\
\hline & & -3589 & 97 & 1331 & 130 & 3.920676 & 45041.190 & 0.070 \\
\hline 148112 & A0p & -216 & 52 & 54 & 96 & 3.04296 & 41803.645 & 0.428 \\
\hline 148199 & B9p & 343 & 69 & 1158 & 94 & 7.72553 & 44771.972 & 0.101 \\
\hline 148330 & A2 & -95 & 42 & 208 & 80 & 4.288404 & 45460.144 & 0.223 \\
\hline 151965 & B9 & -2332 & 98 & 1508 & 145 & 1.60841 & 47683.390 & 0.024 \\
\hline \multirow[t]{2}{*}{152107} & $\mathrm{~A} 3 \mathrm{Vp}$ & 502 & 37 & 296 & 58 & 3.8 & 42554.552 & 0.106 \\
\hline & & 1524 & 88 & 130 & 161 & 3.86778 & 33069.585 & 0.500 \\
\hline 153882 & A1p & 276 & 27 & 2009 & 36 & 6.00858 & 34524.526 & 0.018 \\
\hline 164258 & A3p & -11 & 140 & 1053 & 229 & 0.86281 & 44769.834 & 0.025 \\
\hline 168733 & B8p & -630 & 31 & 345 & 5 & 14.7 & 41428.254 & 0.282 \\
\hline \multirow[t]{2}{*}{170000} & $\mathrm{~A} 0 \mathrm{p}$ & 151 & 30 & 327 & 45 & 1.71649 & 40870.326 & 0.035 \\
\hline & & -52 & 115 & 281 & 204 & 1.71649 & 40871.163 & 0.188 \\
\hline 170397 & B9p & 69 & 68 & 920 & 97 & 2.25454 & 42610.580 & 0.058 \\
\hline 170973 & $\mathrm{~A} 0 \mathrm{p}$ & 285 & 147 & 1081 & 311 & 18.52 & 47008.207 & 0.784 \\
\hline 173650 & $\mathrm{~A} 0 \mathrm{p}$ & -97 & 108 & 309 & 174 & 9.9754 & 33108.446 & 1.016 \\
\hline \multirow[t]{2}{*}{174638} & B7Ve & -1291 & 35 & 498 & 48 & 12.935 & 44008.693 & 0.195 \\
\hline & & -1 & 6 & 43 & 8 & 12.935 & 44008.736 & 0.456 \\
\hline \multirow[t]{2}{*}{175362} & B6IVp & -1286 & 64 & 5376 & 92 & 3.67375 & 44637.022 & 0.010 \\
\hline & & -292 & 84 & 4697 & 121 & 3.67375 & 44637.107 & 0.017 \\
\hline 187474 & $\mathrm{~A} 0 \mathrm{p}$ & -84 & 30 & 1855 & 38 & 2342 . & 36180.231 & 9.961 \\
\hline \multirow[t]{2}{*}{188041} & A6p & 2759 & 41 & 1030 & 57 & 223.826 & 34801.337 & 2.079 \\
\hline & & 765 & 42 & 257 & 56 & 223.826 & 34807.593 & 8.243 \\
\hline 189849 & A4III & 180 & 7 & 131 & 10 & 6.88449 & 39713.875 & 0.122 \\
\hline 192678 & A2 & 1352 & 23 & 415 & 32 & 12.91049 & 36116.531 & 0.164 \\
\hline 194093 & F8Ib & 30 & 7 & 49 & 10 & 1.03474 & 41152.232 & 0.032 \\
\hline 196178 & $B 8 p$ & -1018 & 74 & 859 & 136 & 1.91645 & 42590.381 & 0.030 \\
\hline 196502 & $\mathrm{~A} 2 \mathrm{p}$ & 43 & 43 & 472 & 61 & 20.2747 & 33942.384 & 0.368 \\
\hline 201091 & K5V & -4 & 1 & 7 & 1 & 36.59 & 50972.715 & 0.710 \\
\hline 201601 & A9p & -234 & 15 & 779 & 15 & 27027. & 22098.81 & 11.222 \\
\hline 207260 & A2Ia & 501 & 27 & 589 & 33 & 1688.9 & 41065.746 & 21.445 \\
\hline \multirow[t]{2}{*}{215441} & B9 & 14417 & 235 & 4109 & 376 & 10180. & 36064.62 & 24.480 \\
\hline & & 16452 & 393 & 4671 & 530 & 9.4871 & 36765.450 & 0.187 \\
\hline 220825 & A1p & -164 & 72 & 389 & 101 & 1.14077 & 43739.058 & 0.054 \\
\hline 223640 & B9p & 501 & 36 & 572 & 54 & 3.735239 & 43738.765 & 0.053 \\
\hline 343872 & Ap & 1867 & 70 & 1709 & 99 & 8.8 & 51764.034 & 0.081 \\
\hline
\end{tabular}


V. D. Bychkov et al.: A catalog of stellar magnetic rotational phase curves, Online Material p 5

Table 2. Parameter $r$ of the dipole magnetic field. Asterisk in column 1 indicates that the phase curve of this star was fitted by a double wave. Method: Met $-B_{\mathrm{e}}$ from metal lines, $\mathrm{Hl}-B_{\mathrm{e}}$ from hydrogen lines, all - both previous methods.

\begin{tabular}{|c|c|c|c|c|c|c|c|c|}
\hline HD & $N$ & $\sigma$ & $r$ & $\overline{\sigma_{r}}$ & $N_{V}$ & $\chi^{2}$ & Method & References \\
\hline 2453 & 28 & 16 & 0.443 & 0.030 & 25 & 1.86 & Met & $1,26,256$ \\
\hline 3360 & 118 & 3 & -0.189 & 0.105 & 115 & 1.00 & Met & 360 \\
\hline 3980 & 8 & 252 & -0.921 & 0.040 & 5 & 70.98 & Met & 121 \\
\hline 4778 & 23 & 77 & -0.764 & 0.121 & 20 & 1.01 & $\mathrm{Hl}$ & 171 \\
\hline 5737 & 24 & 25 & -0.583 & 0.095 & 21 & 1.35 & $\mathrm{Hl}$ & $37,181,256$ \\
\hline 8441 & 18 & 61 & -0.357 & 0.148 & 15 & 1.40 & all & 1,237 \\
\hline $9996^{*}$ & 62 & 58 & -0.711 & 0.055 & 59 & 44.21 & all & $1,19,208,209,270,327$ \\
\hline 10783 & 36 & 139 & 0.115 & 0.157 & 33 & 1.36 & Met & 1,35 \\
\hline 11503 & 18 & 48 & -0.479 & 0.056 & 15 & 2.52 & $\mathrm{Hl}$ & 2 \\
\hline 12288 & 21 & 98 & 0.171 & 0.034 & 18 & 11.65 & all & 312,350 \\
\hline 12447 & 22 & 29 & -0.810 & 0.099 & 19 & 0.92 & $\mathrm{Hl}$ & 2 \\
\hline 12767 & 8 & 26 & -0.743 & 0.155 & 5 & 0.91 & $\mathrm{Hl}$ & 2 \\
\hline 14437 & 36 & 69 & 0.451 & 0.031 & 33 & 4.11 & all & 312 \\
\hline 15144 & 57 & 20 & 0.727 & 0.100 & 54 & 0.28 & Met & 1,87 \\
\hline 16582 & 13 & 166 & -0.508 & 0.250 & 10 & 114.05 & all & $77,96,327$ \\
\hline \multirow[t]{2}{*}{18296} & 51 & 68 & -0.337 & 0.347 & 48 & 1.63 & Met & $1,14,327$ \\
\hline & 19 & 31 & -0.618 & 0.259 & 16 & 0.96 & $\mathrm{Hl}$ & $2,197,243$ \\
\hline 19832 & 11 & 49 & -0.710 & 0.181 & 8 & 0.73 & $\mathrm{Hl}$ & 2 \\
\hline 21699 & 17 & 97 & -0.803 & 0.116 & 14 & 4.48 & all & 40,252 \\
\hline 22470 & 14 & 51 & -0.819 & 0.102 & 11 & 1.17 & $\mathrm{Hl}$ & 25,37 \\
\hline 24155 & 6 & 84 & -0.280 & 0.169 & 3 & 0.71 & $\mathrm{Hl}$ & 230 \\
\hline 24712 & 38 & 39 & 0.199 & 0.048 & 35 & 11.61 & all & $21,111,120,184,195,256,310,327$ \\
\hline 25267 & 7 & 24 & 0.017 & 0.181 & 4 & 0.85 & $\mathrm{Hl}$ & 2 \\
\hline 25354 & 5 & 20 & 0.349 & 0.379 & 2 & 0.01 & Met & 1 \\
\hline 25823 & 20 & 66 & -0.214 & 0.233 & 17 & 0.38 & Met & 1,71 \\
\hline 27309 & 13 & 211 & 0.047 & 0.110 & 10 & 2.46 & all & $2,41,142$ \\
\hline 27962 & 16 & 75 & 0.048 & 0.079 & 13 & 1.25 & all & $1,77,98$ \\
\hline 28843 & 5 & 69 & -0.670 & 0.246 & 2 & 0.77 & $\mathrm{Hl}$ & 37 \\
\hline 30466 & 10 & 198 & -0.001 & 0.100 & 7 & 4.95 & Met & $1,44,137$ \\
\hline $32633^{*}$ & 120 & 114 & -0.499 & 0.015 & 117 & 14.09 & all & $1,2,8,175,184,310,327$ \\
\hline 34452 & 11 & 41 & -0.371 & 0.108 & 8 & 0.47 & $\mathrm{Hl}$ & 2,230 \\
\hline 35298 & 5 & 151 & -0.683 & 0.097 & 2 & 1.15 & $\mathrm{Hl}$ & 201 \\
\hline 35456 & 13 & 144 & -0.844 & 0.077 & 10 & 3.38 & all & 201,327 \\
\hline 35502 & 6 & 49 & 0.040 & 0.076 & 3 & 0.21 & $\mathrm{Hl}$ & 201 \\
\hline 35912 & 10 & 66 & -0.636 & 0.103 & 7 & 0.66 & Met & 55,327 \\
\hline 36313 & 6 & 202 & -0.744 & 0.157 & 3 & 1.90 & $\mathrm{Hl}$ & 201 \\
\hline 36485 & 7 & 43 & 0.391 & 0.096 & 4 & 0.22 & all & 135,256 \\
\hline 36526 & 6 & 92 & -0.192 & 0.079 & 3 & 0.40 & $\mathrm{Hl}$ & 201 \\
\hline 36629 & 8 & 75 & -0.618 & 0.182 & 5 & 0.36 & Met & 53,55 \\
\hline 36668 & 6 & 161 & -0.740 & 0.174 & 3 & 1.26 & $\mathrm{Hl}$ & 201 \\
\hline 37017 & 40 & 70 & 0.124 & 0.037 & 37 & 1.99 & $\mathrm{Hl}$ & 24,135 \\
\hline 37022 & 30 & 89 & -0.603 & 0.217 & 27 & 1.81 & Met & 286,348 \\
\hline 37041 & 7 & 77 & -0.084 & 0.113 & 4 & 0.54 & $\mathrm{Hl}$ & 69,75 \\
\hline 37058 & 10 & 91 & -0.293 & 0.066 & 7 & 0.92 & all & $37,53,55,256$ \\
\hline 37140 & 6 & 165 & -0.477 & 0.395 & 3 & 1.03 & $\mathrm{Hl}$ & 201 \\
\hline 37151 & 5 & 42 & -0.566 & 0.320 & 2 & 0.14 & $\mathrm{Hl}$ & 201,204 \\
\hline 37210 & 4 & 184 & -0.415 & 0.370 & 1 & 1.57 & $\mathrm{Hl}$ & 201 \\
\hline 37479 & 22 & 110 & -0.546 & 0.051 & 19 & 1.98 & $\mathrm{Hl}$ & 28,135 \\
\hline 37642 & 6 & 327 & -0.811 & 0.104 & 3 & 5.91 & $\mathrm{Hl}$ & 201 \\
\hline $37776^{*}$ & 51 & 106 & - & - & 46 & 4.55 & $\mathrm{Hl}$ & 24,174 \\
\hline $40312^{*}$ & 30 & 11 & -0.619 & 0.044 & 27 & 2.42 & all & $2,60,310$ \\
\hline 45348 & 26 & 32 & -0.062 & 0.050 & 23 & 5.59 & Met & 42,273 \\
\hline 49333 & 8 & 76 & -0.780 & 0.143 & 5 & 0.79 & $\mathrm{Hl}$ & 37,159 \\
\hline 49606 & 11 & 33 & -0.318 & 0.192 & 8 & 29.88 & all & $230,267,330$ \\
\hline 49976 & 22 & 172 & -0.825 & 0.102 & 19 & 3.62 & Met & $1,91,92,184$ \\
\hline 51418 & 11 & 49 & -0.408 & 0.312 & 8 & 0.23 & Met & 29 \\
\hline 54118 & 14 & 63 & -0.924 & 0.054 & 11 & 2.09 & $\mathrm{Hl}$ & 81,230 \\
\hline
\end{tabular}


V. D. Bychkov et al.: A catalog of stellar magnetic rotational phase curves, Online Material p 6

Table 2. continued.

\begin{tabular}{|c|c|c|c|c|c|c|c|c|}
\hline $\mathrm{HD}$ & $N$ & $\sigma$ & $r$ & $\sigma_{r}$ & $N_{V}$ & $\chi^{2}$ & Method & References \\
\hline 55719 & 31 & 84 & 0.097 & 0.165 & 28 & 0.68 & Met & $32,93,256$ \\
\hline 58260 & 10 & 57 & 0.807 & 0.079 & 7 & 0.58 & $\mathrm{Hl}$ & 24,135 \\
\hline $62140^{*}$ & 36 & 93 & -0.934 & 0.047 & 33 & 6.01 & Met & $62,91,310$ \\
\hline $62140 *$ & 14 & 43 & -0.938 & 0.017 & 11 & 10.78 & Met & 310 \\
\hline 64740 & 21 & 31 & -0.496 & 0.044 & 18 & 1.73 & $\mathrm{Hl}$ & 24,135 \\
\hline \multirow[t]{2}{*}{$65339^{*}$} & 10 & 113 & -0.854 & 0.010 & 7 & 26.34 & Met & 310 \\
\hline & 48 & 117 & -0.959 & 0.027 & 45 & 3.63 & $\mathrm{Hl}$ & $25,33,74,96,190,327$ \\
\hline 71866 & 10 & 32 & -0.802 & 0.023 & 7 & 2.60 & Met & 310 \\
\hline 72968 & 24 & 61 & -0.733 & 0.208 & 21 & 10.20 & Met & $1,91,327$ \\
\hline 73340 & 5 & 87 & 0.307 & 0.091 & 2 & 1.59 & $\mathrm{Hl}$ & 230 \\
\hline 74521 & 12 & 75 & 0.660 & 0.108 & 9 & 22.90 & all & $184,230,327,333$ \\
\hline 77350 & 9 & 35 & -0.456 & 0.164 & 6 & 0.79 & all & $230,327,333$ \\
\hline 78316 & 32 & 30 & -0.611 & 0.280 & 29 & 2.48 & Met & $1,11,330,333$ \\
\hline 79158 & 27 & 51 & -0.910 & 0.062 & 24 & 1.45 & $\mathrm{Hl}$ & $37,181,327$ \\
\hline 83368 & 22 & 80 & -0.893 & 0.078 & 19 & 1.85 & all & $184,186,256$ \\
\hline 89822 & 12 & 39 & -0.331 & 0.338 & 9 & 3.26 & all & $1,2,20,230,237,330$ \\
\hline 90044 & 11 & 61 & -0.863 & 0.098 & 8 & 0.49 & all & 230,324 \\
\hline 90569 & 8 & 14 & -0.399 & 0.397 & 5 & 0.01 & Met & 1 \\
\hline 92664 & 19 & 35 & 0.163 & 0.050 & 16 & 1.64 & $\mathrm{Hl}$ & 230 \\
\hline 96446 & 15 & 68 & 0.354 & 0.075 & 12 & 2.41 & all & 24,184 \\
\hline 96707 & 21 & 51 & -0.602 & 0.153 & 18 & 0.81 & all & 260,324 \\
\hline 98088 & 19 & 87 & -0.743 & 0.157 & 16 & 1.41 & Met & $1,108,310$ \\
\hline 103192 & 5 & 17 & 0.301 & 0.236 & 2 & 0.26 & $\mathrm{Hl}$ & 2,230 \\
\hline 108662 & 53 & 50 & -0.142 & 0.073 & 50 & 1.76 & Met & $1,11,118,333$ \\
\hline 108945 & 13 & 59 & -0.408 & 0.119 & 10 & 0.72 & all & $2,327,333$ \\
\hline 110379 & 16 & 26 & -0.667 & 0.109 & 13 & 2.19 & all & $1,61,77,327,333$ \\
\hline 111133 & 25 & 38 & 0.270 & 0.069 & 22 & 0.73 & Met & $1,23,142$ \\
\hline \multirow[t]{2}{*}{112185} & 24 & 9 & -0.509 & 0.166 & 21 & 1.02 & $\mathrm{Hl}$ & $2,182,271,334$ \\
\hline & 12 & 4 & -0.299 & 0.136 & 9 & 0.49 & Met & 310,334 \\
\hline 112381 & 5 & 71 & 0.777 & 0.082 & 2 & 1.37 & $\mathrm{Hl}$ & 230 \\
\hline \multirow[t]{3}{*}{$112413^{*}$} & 18 & 36 & -0.629 & 0.025 & 15 & 8.46 & Met & 310 \\
\hline & 45 & 51 & -0.700 & 0.028 & 42 & 11.75 & $\mathrm{Hl}$ & $25,33,77,126,215,327$ \\
\hline & 21 & 45 & -0.670 & 0.037 & 18 & 2.90 & $\mathrm{Hl}$ & 33 \\
\hline 116458 & 32 & 52 & 0.689 & 0.037 & 29 & 5.74 & Met & $93,184,187,256$ \\
\hline \multirow[t]{2}{*}{$118022 *$} & 18 & 7 & 0.140 & 0.018 & 15 & 0.71 & Met & 310 \\
\hline & 14 & 53 & 0.163 & 0.126 & 11 & 1.24 & $\mathrm{Hl}$ & $2,76,77$ \\
\hline 119213 & 9 & 71 & -0.096 & 0.098 & 6 & .90 & $\mathrm{Hl}$ & 147 \\
\hline $119419 *$ & 29 & 100 & -0.595 & 0.043 & 26 & 3.81 & all & $168,184,230$ \\
\hline 122532 & 24 & 66 & -0.919 & 0.057 & 21 & 2.16 & all & $168,184,230$ \\
\hline 124224 & 36 & 47 & -0.510 & 0.095 & 20 & 1.51 & $\mathrm{Hl}$ & $2,25,60$ \\
\hline \multirow[t]{2}{*}{125248} & 16 & 129 & -0.931 & 0.051 & 13 & 1.95 & $\mathrm{Hl}$ & 2,25 \\
\hline & 35 & 52 & -0.934 & 0.034 & 32 & 3.90 & Met & $184,239,256,324,333$ \\
\hline \multirow[t]{2}{*}{125823} & 19 & 86 & -0.766 & 0.157 & 16 & 1.27 & Met & 176 \\
\hline & 9 & 34 & -0.881 & 0.084 & 6 & 1.57 & $\mathrm{Hl}$ & 37 \\
\hline $126515^{*}$ & 31 & 104 & -0.781 & 0.045 & 28 & 6.38 & all & $1,18,91,184,256,310,324$ \\
\hline 128898 & 15 & 59 & 0.205 & 0.230 & 12 & 2.36 & all & $2,81,184,256$ \\
\hline 131156 & 32 & 31 & -0.058 & 0.141 & 29 & 4.17 & Met & $27,299,311$ \\
\hline \multirow[t]{2}{*}{133029} & 18 & 110 & 0.417 & 0.056 & 15 & 2.55 & $\mathrm{Hl}$ & 2,326 \\
\hline & 91 & 52 & 0.801 & 0.058 & 88 & 0.94 & Met & 1,94 \\
\hline 133652 & 9 & 77 & -0.703 & 0.056 & 6 & 1.79 & $\mathrm{Hl}$ & 230 \\
\hline $133880 *$ & 12 & 201 & -0.848 & 0.027 & 9 & 21.56 & $\mathrm{Hl}$ & 172 \\
\hline 134793 & 5 & 86 & -0.612 & 0.239 & 2 & 1.17 & Met & 1 \\
\hline $137509 *$ & 16 & 186 & -0.689 & 0.163 & 13 & 5.13 & all & $184,230,256$ \\
\hline \multirow[t]{5}{*}{137909} & 36 & 17 & -0.574 & 0.029 & 33 & 8.73 & $\mathrm{Hl}$ & 2,76 \\
\hline & 17 & 3 & -0.737 & 0.013 & 14 & 0.35 & Met & 310 \\
\hline & 14 & 32 & -0.921 & 0.006 & 11 & 69.00 & Met & 31,327 \\
\hline & 34 & 48 & -0.877 & 0.074 & 31 & 2.18 & Met & 63 \\
\hline & 33 & 32 & -0.660 & 0.055 & 30 & 2.65 & Met & 63 \\
\hline 137949 & 18 & 76 & 0.277 & 0.083 & 15 & 3.58 & Met & $1,26,91,256$ \\
\hline
\end{tabular}


Table 2. continued.

\begin{tabular}{|c|c|c|c|c|c|c|c|c|}
\hline $\mathrm{HD}$ & $N$ & $\sigma$ & $r$ & $\sigma_{r}$ & $N_{V}$ & $\chi^{2}$ & Method & References \\
\hline 140160 & 10 & 195 & -0.366 & 0.273 & 7 & 4.77 & all & $2,25,333$ \\
\hline $142301 *$ & 20 & 131 & -0.430 & 0.035 & 17 & 3.33 & $\mathrm{Hl}$ & 89 \\
\hline 142990 & 18 & 70 & -0.438 & 0.074 & 15 & 1.49 & $\mathrm{Hl}$ & 37,230 \\
\hline 143473 & 6 & 20 & 0.530 & 0.090 & 3 & 0.03 & all & 184,230 \\
\hline 144334 & 12 & 91 & -0.194 & 0.095 & 9 & 1.79 & $\mathrm{Hl}$ & 37 \\
\hline \multirow[t]{2}{*}{$147010 *$} & 12 & 86 & 0.595 & 0.040 & 9 & 1.05 & $\mathrm{Hl}$ & 168 \\
\hline & 19 & 114 & 0.460 & 0.039 & 16 & 1.90 & Met & 184,256 \\
\hline 148112 & 12 & 43 & 0.395 & 0.226 & 9 & 1.52 & all & $2,327,333$ \\
\hline 148199 & 13 & 88 & -0.544 & 0.078 & 10 & 1.71 & $\mathrm{Hl}$ & 168,230 \\
\hline 148330 & 13 & 60 & -0.362 & 0.269 & 10 & 5.52 & Met & $179,327,333$ \\
\hline 151965 & 9 & 91 & 0.216 & 0.054 & 6 & 1.27 & $\mathrm{Hl}$ & 230 \\
\hline \multirow[t]{2}{*}{152107} & 21 & 39 & 0.262 & 0.102 & 18 & 1.69 & $\mathrm{Hl}$ & $2,220,290$ \\
\hline & 28 & 44 & 0.790 & 0.096 & 25 & 0.24 & Met & $1,83,178,219,290,327$ \\
\hline 153882 & 28 & 113 & -0.759 & 0.020 & 25 & 99.26 & Met & $31,72,184,256,310$ \\
\hline 164258 & 12 & 123 & -0.803 & 0.136 & 9 & 0.70 & Met & 41,327 \\
\hline 168733 & 24 & 39 & 0.306 & 0.083 & 19 & 1.36 & all & $86,184,256$ \\
\hline \multirow[t]{2}{*}{170000} & 15 & 49 & -0.369 & 0.106 & 12 & 2.60 & $\mathrm{Hl}$ & $60-\mathrm{H}$ bet.only \\
\hline & 18 & 92 & -0.516 & 0.323 & 15 & 0.65 & $\mathrm{Hl}$ & 25 - H Alp.only \\
\hline 170397 & 12 & 25 & -0.845 & 0.104 & 9 & 0.32 & all & $2,184,230$ \\
\hline 170973 & 5 & 27 & -0.580 & 0.179 & 2 & 0.07 & all & 184,230 \\
\hline 173650 & 24 & 51 & -0.466 & 0.326 & 21 & 0.24 & Met & 1 \\
\hline \multirow[t]{2}{*}{174638} & 63 & 57 & 0.444 & 0.038 & 60 & 2.39 & Met & $191,227,342,343$ \\
\hline & 28 & 13 & -0.811 & 0.139 & 25 & 7.66 & Met & 344 \\
\hline \multirow[t]{2}{*}{$175362 *$} & 12 & 224 & -0.614 & 0.015 & 9 & 16.06 & $\mathrm{Hl}$ & 37 \\
\hline & 30 & 157 & -0.884 & 0.031 & 27 & 7.76 & Met & 184,256 \\
\hline 187474 & 56 & 25 & -0.914 & 0.029 & 53 & 1.61 & Met & $1,184,256$ \\
\hline \multirow[t]{2}{*}{188041} & 53 & 71 & 0.457 & 0.022 & 50 & 3.05 & Met & 4 \\
\hline & 44 & 49 & 0.500 & 0.091 & 41 & 1.96 & Met & $1,15,16,184,256$ \\
\hline 189849 & 60 & 22 & 0.159 & 0.041 & 57 & 14.22 & Met & $10,97,98,327$ \\
\hline 192678 & 31 & 36 & 0.531 & 0.028 & 28 & 3.99 & all & $1,41,255$ \\
\hline 194093 & 94 & 10 & -0.234 & 0.147 & 91 & 9.37 & Met & $76,140,189,229$ \\
\hline 196178 & 11 & 70 & 0.089 & 0.075 & 8 & 17.68 & all & 2,327 \\
\hline 196502 & 56 & 53 & -0.817 & 0.111 & 53 & 9.27 & all & $1,7,22,190,327$ \\
\hline 201091 & 15 & & -0.304 & 0.068 & 12 & 4.86 & Met & 354 \\
\hline 201601 & 208 & 17 & -0.539 & 0.024 & 205 & 15.74 & all & $1,2,46,47,48,49,105,146,184,195,268,256,327$ \\
\hline $207260 *$ & 157 & 43 & -0.080 & 0.035 & 154 & 4.38 & all & $136,194,325$ \\
\hline \multirow[t]{2}{*}{215441} & 48 & 560 & 0.557 & 0.028 & 45 & 35.12 & all & $5,30,190,224$ \\
\hline & 20 & 391 & 0.559 & 0.041 & 17 & 1.68 & $\mathrm{Hl}$ & 30,190 \\
\hline 220825 & 13 & 22 & -0.415 & 0.191 & 10 & 0.14 & all & $2,25,327$ \\
\hline 223640 & 27 & 51 & -0.064 & 0.054 & 24 & 1.95 & $\mathrm{Hl}$ & 224 \\
\hline $343872 *$ & 17 & 167 & 0.044 & 0.039 & 14 & 9.12 & Met & 329 \\
\hline
\end{tabular}


V. D. Bychkov et al.: A catalog of stellar magnetic rotational phase curves, Online Material p 8

Table 3. Double wave $B_{\mathrm{e}}$ variations.

\begin{tabular}{|c|c|c|c|c|c|c|c|c|c|c|c|c|c|c|}
\hline $\mathrm{HD}$ & $B_{0}$ & $\sigma_{B_{0}}$ & $B_{1}$ & $\sigma_{B_{1}}$ & $B_{2}$ & $\sigma_{B_{2}}$ & $z_{1}$ & $\sigma_{Z_{1}}$ & $z_{2}$ & $\sigma_{Z_{2}}$ & $N_{V}$ & $\chi^{2}$ & Method & References \\
\hline 9996 & -252 & 34 & 855 & 59 & 281 & 42 & 0.943 & 0.008 & 0.096 & 0.028 & 57 & 56.59 & all & $1,19,208,209,270,327$ \\
\hline \multirow[t]{3}{*}{32633} & -1083 & 40 & 3026 & 45 & 1279 & 61 & 0.100 & 0.005 & 0.877 & 0.008 & 115 & 13.80 & all & $1,2,8,175,184,310,327$ \\
\hline & -1338 & 54 & 2626 & 63 & 703 & 72 & 0.066 & 0.005 & 0.859 & 0.012 & 10 & 1.64 & Met & 184,310 \\
\hline & -1460 & 109 & 2784 & 165 & 1125 & 153 & 0.083 & 0.009 & 0.909 & 0.024 & 12 & 1.26 & $\mathrm{Hl}$ & 2 \\
\hline 37776 & -240 & 62 & 308 & 87 & 1347 & 87 & 0.962 & 0.052 & 0.900 & 0.011 & 39 & 5.00 & $\mathrm{Hl}$ & 174 \\
\hline 40312 & 83 & 12 & 248 & 19 & 62 & 17 & 0.017 & 0.013 & 0.023 & 0.062 & 6 & 1.61 & Met & 310 \\
\hline 62140 & -63 & 14 & 1638 & 21 & 34 & 150 & 0.002 & 0.002 & 0.052 & 0.227 & 9 & 7.82 & Met & 310 \\
\hline 65339 & -375 & 26 & 4382 & 38 & 316 & 39 & 0.005 & 0.001 & 0.014 & 0.018 & 5 & 15.83 & Met & 310 \\
\hline \multirow[t]{2}{*}{112413} & -159 & 12 & 704 & 16 & 193 & 19 & 0.000 & 0.004 & 0.994 & 0.016 & 13 & 1.28 & Met & 310 \\
\hline & -184 & 20 & 1014 & 31 & 94 & 22 & 0.999 & 0.005 & 0.927 & 0.058 & 40 & 10.93 & $\mathrm{Hl}$ & $25,33,77,126,215,327$ \\
\hline 118022 & -556 & 9 & 422 & 14 & 28 & 16 & 0.999 & 0.005 & 0.962 & 0.090 & 13 & 0.49 & Met & 310 \\
\hline 119419 & -590 & 76 & 2443 & 114 & 520 & 149 & 0.003 & 0.007 & 0.164 & 0.053 & 24 & 2.85 & all & $168,184,230$ \\
\hline 126515 & -293 & 62 & 2043 & 84 & 536 & 214 & 0.991 & 0.008 & 0.183 & 0.080 & 26 & 1.77 & all & $1,18,91,184,256,310,324$ \\
\hline 133880 & -419 & 52 & 3177 & 78 & 918 & 65 & 0.004 & 0.003 & 0.039 & 0.012 & 7 & 4.07 & $\mathrm{Hl}$ & 172 \\
\hline 137509 & 243 & 119 & 978 & 152 & 960 & 144 & 0.986 & 0.029 & 0.909 & 0.029 & 11 & 1.74 & all & $184,230,256$ \\
\hline 142301 & -957 & 103 & 3013 & 165 & 624 & 281 & 0.001 & 0.007 & 0.165 & 0.082 & 15 & 1.41 & $\mathrm{Hl}$ & 89 \\
\hline \multirow[t]{2}{*}{147010} & -4702 & 97 & 1268 & 149 & 429 & 189 & 0.990 & 0.018 & 0.933 & 0.069 & 7 & 0.44 & $\mathrm{Hl}$ & 168 \\
\hline & -3646 & 100 & 1350 & 130 & 407 & 121 & 0.019 & 0.019 & 0.990 & 0.074 & 14 & 1.38 & Met & 184,256 \\
\hline \multirow[t]{2}{*}{175362} & -1436 & 67 & 5434 & 92 & 1097 & 98 & 0.006 & 0.003 & 0.888 & 0.012 & 7 & 2.16 & $\mathrm{Hl}$ & 37 \\
\hline & -299 & 84 & 4591 & 132 & 859 & 119 & 0.005 & 0.005 & 0.891 & 0.030 & 25 & 3.48 & Met & 184,256 \\
\hline 207260 & 430 & 32 & 597 & 36 & 268 & 54 & 0.975 & 0.013 & 0.941 & 0.022 & 152 & 5.52 & all & $136,194,325$ \\
\hline 343872 & 1777 & 73 & 1904 & 102 & 917 & 104 & 0.979 & 0.009 & 0.829 & 0.017 & 12 & 2.38 & Met & 329 \\
\hline
\end{tabular}


Table 4. Cross-reference list.

\begin{tabular}{|c|c|}
\hline 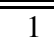 & "Babcock (1958) \\
\hline 2 & Borra \& Landstreet (1980) \\
\hline 4 & Babcock (1954) \\
\hline 5 & Babcock (1960) \\
\hline 7 & Preston (1967b) \\
\hline 8 & Preston \& Stępień (1968a) \\
\hline 10 & Conti (1969) \\
\hline 11 & Preston et al. (1969) \\
\hline 14 & Preston (1969) \\
\hline 15 & Wolff (1969a) \\
\hline 16 & Wolff (1969b) \\
\hline 18 & Preston (1970) \\
\hline 19 & Preston \& Wolff (1970) \\
\hline 20 & Conti (1970a) \\
\hline 21 & Preston (1972) \\
\hline 22 & Wolff \& Bonsack (1972) \\
\hline 23 & Wolff \& Wolff (1972) \\
\hline 24 & Borra \& Landstreet (1979) \\
\hline 25 & Landstreet et al. (1975) \\
\hline 26 & Wolff (1975) \\
\hline 27 & Borra \& Vaughan (1978) \\
\hline 28 & Landstreet \& Borra (1978) \\
\hline 29 & Jones et al. (1974) \\
\hline 30 & Borra \& Landstreet (1978) \\
\hline 31 & Vogt et al. (1980) \\
\hline 32 & Bonsack (1976) \\
\hline 33 & Borra \& Landstreet (1977) \\
\hline 35 & Preston \& Stępień (1968b) \\
\hline 37 & Borra et al. (1983) \\
\hline 40 & Glagolevskij \& Chunakova (1985) \\
\hline 41 & Glagolevskij et al. (1985a) \\
\hline 42 & Rakos et al. (1977) \\
\hline 44 & Huchra (1972) \\
\hline 46 & Bonsack \& Pilachowski (1974) \\
\hline 47 & Brown \& Landstreet (1981) \\
\hline 48 & Scholz (1979) \\
\hline 49 & Scholz (1971b) \\
\hline 53 & Sargent et al. (1967) \\
\hline 55 & Conti (1970b) \\
\hline 60 & Landstreet \& Borra (1977) \\
\hline 61 & Boesgaard (1974) \\
\hline 62 & Bonsack et al. (1974) \\
\hline 63 & Borra \& Vaughan (1977) \\
\hline 69 & Kemp \& Wolstencroft (1973) \\
\hline 71 & Wolff (1973) \\
\hline 72 & Slovak (1982) \\
\hline 74 & Angel et al. (1973) \\
\hline 75 & Borra (1975b) \\
\hline 76 & Borra et al. (1981) \\
\hline 77 & Landstreet (1982) \\
\hline 81 & Borra \& Landstreet (1975) \\
\hline 83 & Wolff \& Preston (1978) \\
\hline 86 & Jones \& Wolff (1974) \\
\hline 87 & Bonsack (1981a) \\
\hline 89 & Landstreet et al. (1979) \\
\hline
\end{tabular}

Table 4. continued.

\begin{tabular}{|c|c|}
\hline 91 & Heuvel \& van den (1971) \\
\hline 92 & Pilachowski et al. (1974) \\
\hline 93 & Wood \& Campusano (1975) \\
\hline 94 & Bonsack (1977) \\
\hline 96 & Rudy \& Kemp (1978) \\
\hline 97 & Kuvshinov (1972) \\
\hline 98 & Kuvshinov et al. (1976) \\
\hline 105 & Scholz (1975) \\
\hline 108 & Hildebrandt et al. (1973) \\
\hline 111 & Gollnow (1964) \\
\hline 118 & Rustamov \& Khotnyanskij (1980) \\
\hline 120 & Gollnow (1971) \\
\hline 121 & Maitzen et al. (1980) \\
\hline 126 & Wolstencroft et al. (1981) \\
\hline 135 & Bohlender et al. (1987) \\
\hline 136 & Scholz \& Gerth (1980) \\
\hline 137 & Rudiger \& Scholz (1988) \\
\hline 140 & Glagolevskij et al. (1989) \\
\hline 142 & Glagolevskij et al. (1982) \\
\hline 146 & Zverko et al. (1989) \\
\hline 147 & Mikulasek et al. (1984) \\
\hline 153 & Romanov et al. (1988) \\
\hline 159 & Ryabchikova et al. (1988) \\
\hline 168 & Thompson et al. (1987) \\
\hline 171 & Bohlender (1989) \\
\hline 172 & Landstreet (1990) \\
\hline 174 & Thompson \& Landstreet (1985) \\
\hline 175 & Renson (1984) \\
\hline 176 & Wolff \& Morrison (1974) \\
\hline 178 & Gerth (1990a) \\
\hline 179 & Ziznovsky \& Romanyuk (1990) \\
\hline 181 & Shore et al. (1990) \\
\hline 182 & Bohlender \& Landstreet (1990a) \\
\hline 184 & Mathys (1991) \\
\hline 186 & Thompson (1983) \\
\hline 187 & Albrecht et al. (1977) \\
\hline 189 & Plachinda (1990) \\
\hline 190 & Weiss et al. (1990) \\
\hline 191 & Skulskij (1990) \\
\hline 194 & Gerth et al. (1991a) \\
\hline 195 & Bychkov et al. (1991) \\
\hline 197 & El'kin et al. (1991) \\
\hline 201 & Borra (1994) \\
\hline 204 & Landstreet (2000) \\
\hline 208 & Scholz (1983) \\
\hline 209 & Scholz (1978) \\
\hline 215 & Shtol' et al. (1992a) \\
\hline 219 & Gerth et al. (1992a) \\
\hline 220 & Bychkov et al. (1992) \\
\hline 224 & El'kin (1992) \\
\hline 227 & Skulskij et al. (1992a) \\
\hline 229 & Skulskij et al. (1992b) \\
\hline 230 & Bohlender et al. (1993) \\
\hline 237 & Plachinda et al. (1993) \\
\hline 239 & Johnstone \& Penston (1987) \\
\hline
\end{tabular}


Table 4. continued.

\begin{tabular}{ll}
\hline \hline 243 & Glagolevskij et al. (1995) \\
252 & Brown et al. (1985) \\
255 & Wade et al. (1996) \\
256 & Mathys \& Hubrig (1997) \\
260 & Bychkov et al. (1997a) \\
267 & Bychkov et al. (1997b) \\
268 & Bychkov \& Shtol' (1997) \\
270 & Bychkov et al. (1997c) \\
271 & El'kin et al. (1997) \\
273 & Weiss (1986) \\
286 & Glagolevskij \& Chuntonov (1998) \\
290 & Gerth (1994) \\
299 & Borra et al. (1984) \\
310 & Wade et al. (2000a) \\
311 & Plachinda \& Tarasova (2000b) \\
312 & Wade et al. (2000b) \\
324 & Leone \& Catanzaro (2001) \\
325 & Verdugo et al. (2002) \\
326 & El'kin (2000) \\
327 & Bychkov \& Bychkova (2002) \\
329 & Elkin et al. (2002) \\
330 & Chuntonov (2001) \\
333 & Shorlin et al. (2002) \\
334 & Monin et al. (2002) \\
342 & Skulskij (1982) \\
343 & Skulskij (1985) \\
344 & Skulskij \& Plachinda (1993) \\
348 & Kudryavtsev et al. (2000) \\
350 & Panchuk et al. (2000) \\
354 & Tarasova et al. (2001) \\
360 & Neiner et al. (2003) \\
\hline &
\end{tabular}


Table 5. Periods of magnetic stars.

\begin{tabular}{|c|c|c|}
\hline HD & $P$ (days) & Reference \\
\hline 2453 & 520.5 & This paper \\
\hline 3360 & 5.370447 & Ref. 360 \\
\hline 3980 & 3.9516 & Maitzen et al. (1980) \\
\hline 4778 & 2.5616 & Bohlender (1989) \\
\hline 5737 & 21.654 & Ref. 181 \\
\hline 8441 & 1.80889 & This paper \\
\hline $9996^{*}$ & 7692. & This paper \\
\hline 10783 & 4.14628 & This paper, it is close to Genderen (1964) \\
\hline 11503 & 1.6093 & Borra \& Landstreet (1980) \\
\hline 12288 & 34.79 & Ref. 312 \\
\hline 12447 & 1.49070 & Ref. 2 \\
\hline 12767 & 1.892 & Ref. 137 \\
\hline 14437 & 28.87 & This paper \\
\hline 15144 & 15.88 & Bonsack (1981b) \\
\hline 16582 & 1.40744 & This paper \\
\hline 18296 & 2.8842 & Preston $(1969 c)$ \\
\hline 19832 & 0.727893 & Stępień \& Czechowski (1993) \\
\hline 21699 & 2.4765 & Brown et al. (1985) \\
\hline 22470 & 0.6785 & Ref. 37 \\
\hline 24155 & 2.53465 & Ref. 230 \\
\hline 24712 & 12.4617 & This paper, it is close to Bagnulo et al. (1995) \\
\hline 25267 & 5.954 & Ref. 2 \\
\hline 25354 & 3.90072 & Schoneich et al. (1976) \\
\hline 25823 & 4.65853 & This paper \\
\hline 27309 & 1.10496 & North \& Adelman (1995) \\
\hline 27962 & 2.13006 & This paper \\
\hline 28843 & 1.373813 & Mathys et al. (1986) \\
\hline 30466 & 1.3900 & Rakosch \& Fiedler (1978) \\
\hline $32633^{*}$ & 6.43000 & Renson (1972) \\
\hline 34452 & 2.4687 & Bohlender et al. (1993) \\
\hline 35298 & 1.85336 & North (1984) \\
\hline 35456 & 0.28313 & This paper \\
\hline 35502 & 1.707 & Borra (1981) \\
\hline 35912 & 0.89786 & This paper \\
\hline 36313 & 0.58931 & This paper \\
\hline 36485 & 2.0129 & This paper \\
\hline 36526 & 1.5405 & North (1984) \\
\hline 36629 & 5.01255 & This paper \\
\hline 36668 & 2.1211 & North (1984) \\
\hline 37017 & 0.901175 & Borra \& Landstreet (1979) \\
\hline 37022 & 15.422 & Stahl et al. (1996) \\
\hline 37041 & 3.14264 & This paper \\
\hline 37058 & 1.022 & This paper \\
\hline 37140 & 2.7088 & North (1984) \\
\hline 37151 & 5.6732 & North (1984) \\
\hline 37210 & 11.0494 & North (1984) \\
\hline 37479 & 1.190811 & Shore \& Brown (1990) \\
\hline 37642 & 1.07977 & North (1984) \\
\hline $37776^{*}$ & 1.538675 & Shore \& Brown (1990) \\
\hline
\end{tabular}


Table 5. continued.

\begin{tabular}{|c|c|c|}
\hline HD & $P$ (days) & Reference \\
\hline $40312^{*}$ & 3.61866 & Ref. 2 \\
\hline 45348 & 6.90 & Weiss (1986) \\
\hline 49333 & 2.18010 & Bohlender et al. (1993) \\
\hline 49606 & 1.10503 & This paper \\
\hline 49976 & 2.97668 & Catalano \& Leone (1994) \\
\hline 51418 & 5.631 & This paper \\
\hline 54118 & 3.27533 & Bohlender et al. (1993) \\
\hline 55719 & 1.00335 & This paper \\
\hline 58260 & 1.657 & Pedersen (1979) \\
\hline $62140^{*}$ & 4.28488 & This paper, it is close to Leroy (1995) \\
\hline 64740 & 1.33026 & Shore \& Brown (1990) \\
\hline $65339^{*}$ & 8.0267 & Musielok \& Madej (1988) \\
\hline 71866 & 6.80024 & Bagnulo et al. (1995) \\
\hline 72968 & 4.66458 & This paper, it is close to Steinitz \& Pyper (1970) \\
\hline 73340 & 2.66753 & Bohlender et al. (1993) \\
\hline 74521 & 7.76851 & Catalano \& Leone (1993) \\
\hline 77350 & 4.024 & Kozlova et al. (1975) \\
\hline 78316 & 5.0035 & Ref. 11 \\
\hline 79158 & 3.8345 & This paper, it is close to Bohlender (1994) \\
\hline 83368 & 2.851962 & Mathys (1991) \\
\hline 89822 & 7.5586 & Catalano \& Leone (1991) \\
\hline 90044 & 4.379 & Ref. 324 \\
\hline 90569 & 1.445 & Wolff \& Morrison (1975) \\
\hline 92664 & 1.67315 & Bohlender et al. (1993) \\
\hline 96446 & 0.85521 & This paper, it is close to Mathews \& Bohlender (1991) \\
\hline 96707 & 2.49183 & This paper \\
\hline 98088 & 5.81714 & This paper, it is close to Catalano \& Leone (1994) \\
\hline 103192 & 2.344 & Manfroid \& Renson (1980) \\
\hline 108662 & 5.0805 & Rice \& Wehlau (1994) \\
\hline 108945 & 1.92442 & This paper, it is close to Zverko (1987) \\
\hline 110379 & 12.92696 & This paper \\
\hline 111133 & 16.3078 & North \& Adelman (1995) \\
\hline 112185 & 5.0887 & Bohlender \& Landstreet (1990) \\
\hline 112381 & 2.84 & Bohlender et al. (1993) \\
\hline 112413 & 5.46939 & Borra \& Vaughan (1978) \\
\hline 116458 & 2.3596 & This paper \\
\hline 118022 & 3.722084 & Catalano \& Leone (1994) \\
\hline 119213 & 2.44997 & Pyper \& Adelman (1985) \\
\hline 119419 & 2.6006 & Bohlender et al. (1993) \\
\hline 122532 & 3.68137 & This paper, it is close to Bohlender et al. (1993) \\
\hline 124224 & 0.520675 & Borra \& Landstreet (1980) \\
\hline 125248 & 9.2954 & Mathys (1994) \\
\hline 125823 & 8.8171 & Pedersen \& Thomsen (1977) \\
\hline 126515 & 129.95 & Mathys et al. (1986) \\
\hline 128898 & 4.4794 & This paper, it is close to Kurtz et al. (1994) \\
\hline 131156 & 6.314 & Plachinda et al. (2000) \\
\hline 133029 & 2.105 & This paper \\
\hline 133652 & 2.3040 & Lanz et al. (1991) \\
\hline 133880 & 0.877485 & Landstreet (1990) \\
\hline 134793 & 2.7800 & Blanco et al. (1978) \\
\hline 137509 & 4.4912 & Lanz et al. (1991) \\
\hline 137909 & 18.4868 & Leroy (1995) \\
\hline
\end{tabular}


V. D. Bychkov et al.: A catalog of stellar magnetic rotational phase curves, Online Material p 13

Table 5. continued.

\begin{tabular}{ccl}
\hline \hline HD & $P$ (days) & Reference \\
\hline 137949 & 11.13313 & This paper \\
140160 & 1.59584 & Musielok et al. (1980) \\
142301 & 1.45955 & Landstreet et al. (1979) \\
142990 & 0.9791 & Catalano \& Leone (1996) \\
143473 & 2.84263 & This paper \\
144334 & 1.49497 & North (1987) \\
147010 & 3.920676 & Catalano \& Leone (1993) \\
148112 & 3.04296 & Hatzes (1991) \\
148199 & 7.72553 & Bohlender et al. (1993) \\
148330 & 4.288404 & Ziznovsy \& Romanyuk (1990) \\
151965 & 1.60841 & Lanz \& Mathys (1991b) \\
152107 & 3.86778 & This paper, it is close to Gerth (1990b) \\
153882 & 6.00858 & This paper, it is close to Mathys (1991) \\
164258 & 0.86281 & This paper, it is close to Catalano \& Leone (1994) \\
168733 & 14.78437 & This paper, it is close to Jones \& Wolff (1974) \\
170000 & 1.71649 & Musielok \& Madej (1988) \\
170397 & 2.25454 & This paper, it is close to Borra \& Landstreet (1980) \\
170973 & 18.52 & This paper, it is close to Bohlender et al. (1993) \\
173650 & 9.9754 & Hildebrandt et al. (1985) \\
174638 & 12.935 & Skulskij (1982) \\
175362 & 3.67375 & Catalano \& Leone (1996) \\
187474 & 2342. & This paper, it is close to Hensberge (1993) \\
188041 & 223.826 & This paper, it is close to Hensberge (1993) \\
189849 & 6.88449 & This paper \\
192678 & 12.91049 & This paper \\
194093 & 1.03474 & Plachinda (1990) \\
196178 & 1.91645 & This paper \\
196502 & 20.2747 & Panov (1976) \\
201091 & 36.59 & Tarasova et al. (2001) \\
201601 & 27027. & This paper \\
207260 & 1688.9 & This paper \\
215441 & 10180. & Leckrone (1974) \\
& 9.4871 & This paper \\
\hline & &
\end{tabular}




\section{Appendix A: Magnetic rotational phase curves}

The Appendix presents the catalogue of magnetic rotational phase curves $B_{\mathrm{e}}(\phi)$ for 136 stars in graphical form. All phase curves were expressed in a homogeneous manner, with the effective magnetic field $B_{\mathrm{e}}$ (in G) vs. magnetic phase $\phi$. Note that in some cases several phase curves correspond to the same star. This occurs in cases when different observational techniques produced significantly different series of $B_{\mathrm{e}}$ values. 
V. D. Bychkov et al.: A catalog of stellar magnetic rotational phase curves, Online Material p 15

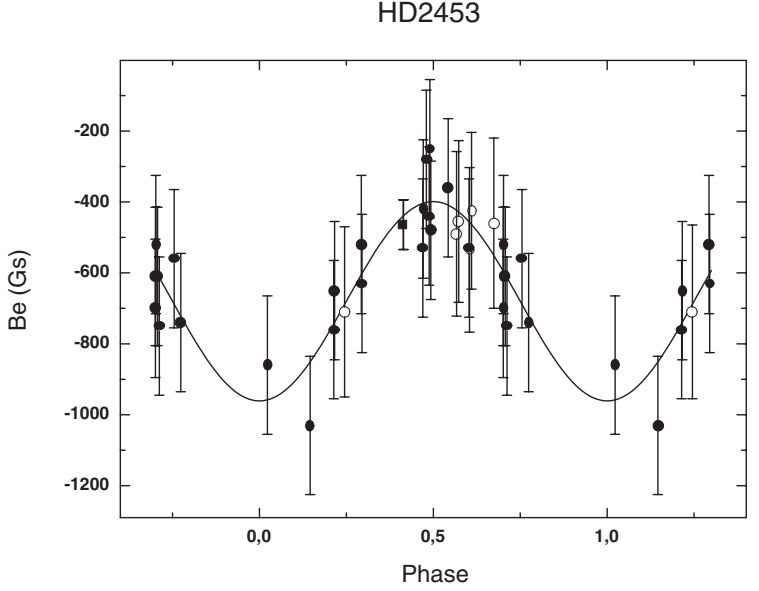

Fig. A.1. HD 2453. Ap star with long period, which is not well determined. Values of $P_{\text {rot }}$ range from $521^{\mathrm{d}}$ up to $547^{\mathrm{d}}$ in various papers. We have found the best period $P_{\text {rot }}=520.5^{\mathrm{d}}$. Open circles - Ref. 1 of our list, filled circles - Ref. 26, filled squares - Ref. 256.

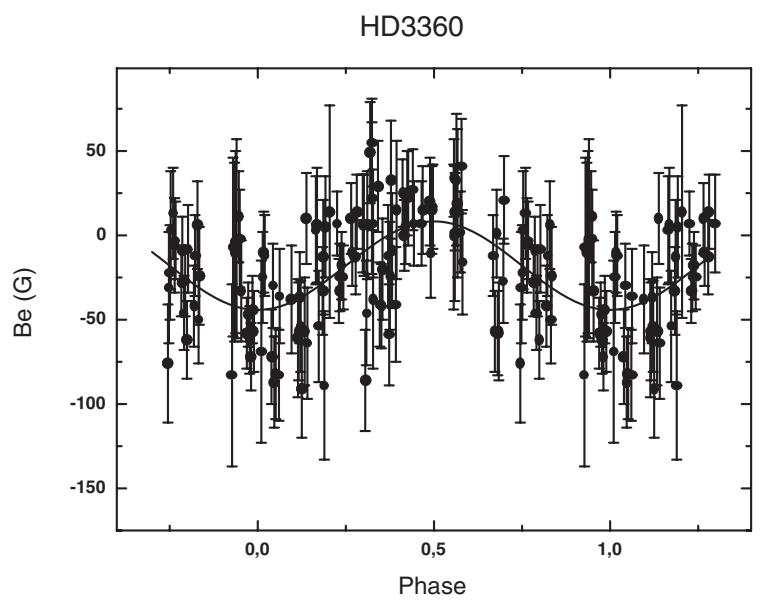

Fig. A.2. HD 3360. Magnetic field $B_{\mathrm{e}}$ was measured in metal lines in Ref. 360, and in hydrogen lines - Ref. 77. Single $B_{\mathrm{e}}$ measurement in Ref. 77 was not used to construct phase curve, due to its low accuracy. Phase curve $B_{\mathrm{e}}(\phi)$ was drawn following Ref. 360 .

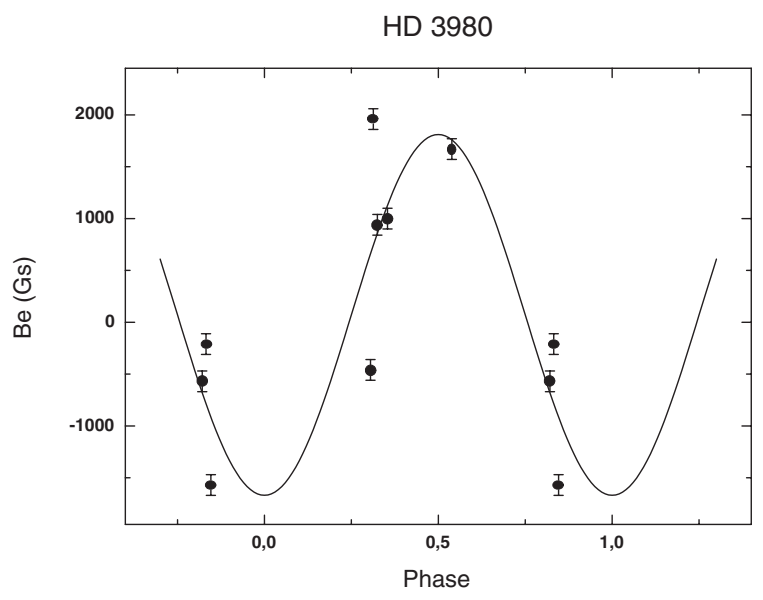

Fig. A.3. HD 3980. The best period is $P_{\mathrm{mag}}=3.9516^{\mathrm{d}}$, which was taken from Maitzen et al. (1980).

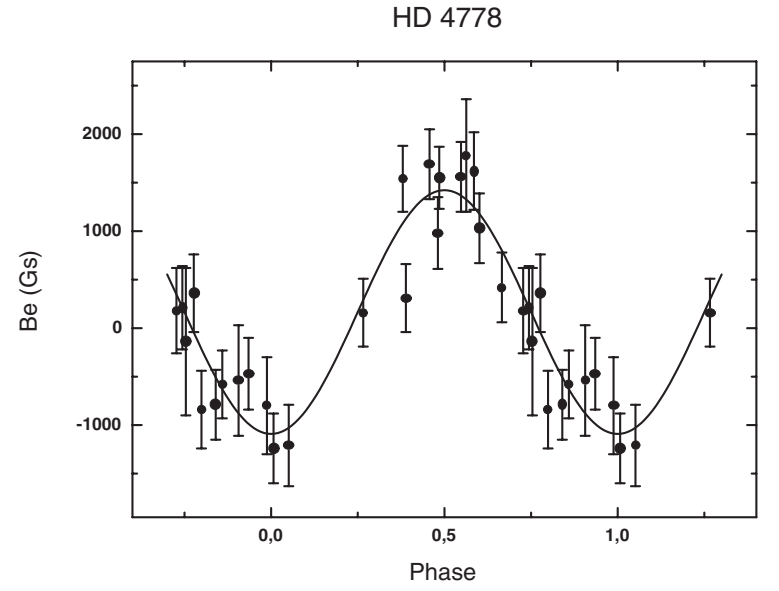

Fig. A.4. HD 4778. We used the period $P=2.5616^{\mathrm{d}}$ taken from Bohlender (1989). Measurements of $B_{\mathrm{e}}$ were done in $\mathrm{H}$ lines, Ref. 171.

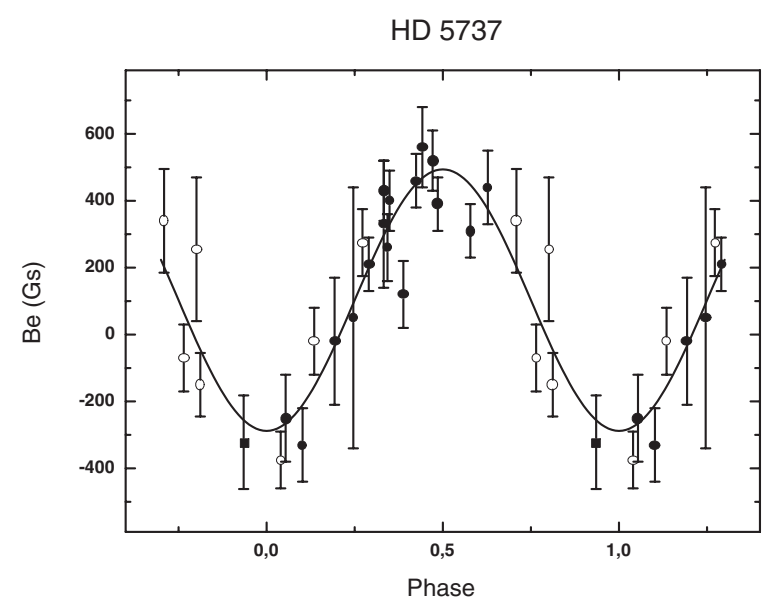

Fig. A.5. HD 5737. Period $P=21.654^{\mathrm{d}}$ was taken from Ref. 181 . Open circles - Ref. 37, filled circles - Ref. 181, filled squares Ref. 256.

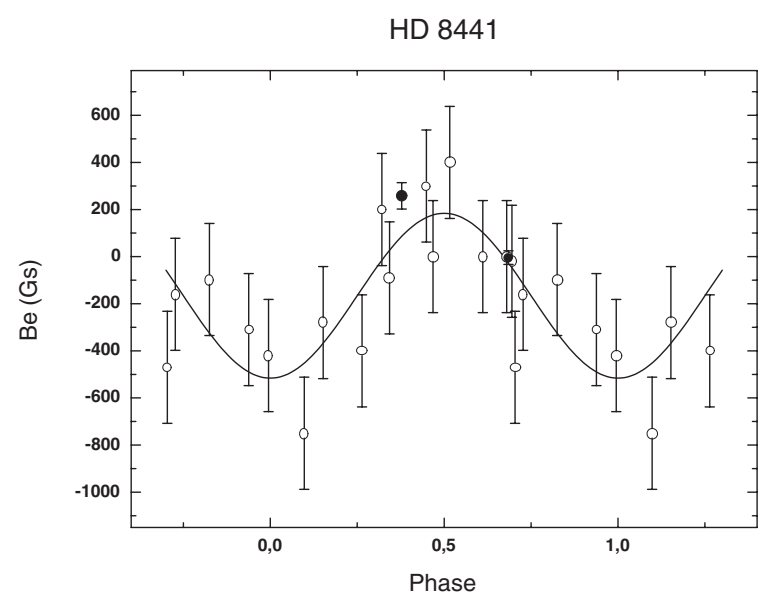

Fig. A.6. HD 8441. We used the compilation of published $B_{\mathrm{e}}$ estimates for the determination of the new period, $P=1.80889^{\mathrm{d}}$. Further $B_{\mathrm{e}}$ observations are necessary to improve this period. Open circles - Ref. 1, filled circles - Ref. 327. 
V. D. Bychkov et al.: A catalog of stellar magnetic rotational phase curves, Online Material p 16

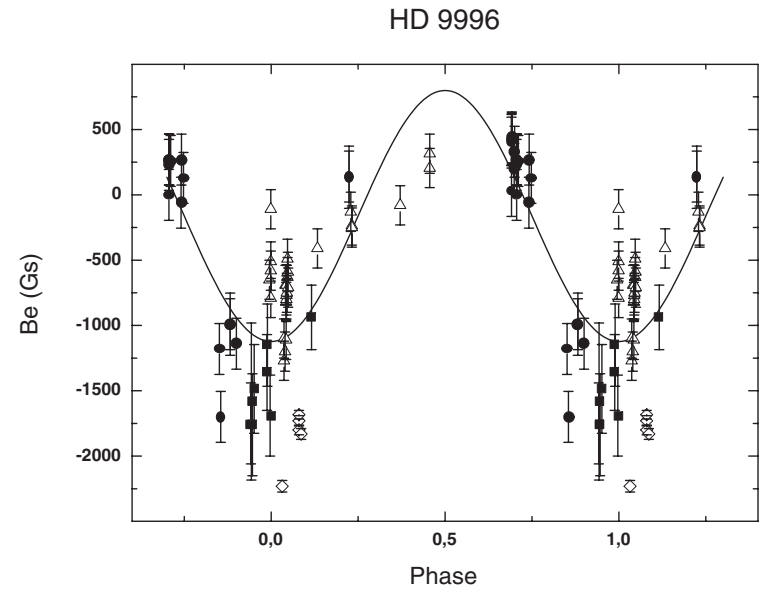

Fig. A.7. HD 9996. We determined the period $P_{\mathrm{mag}}=7692^{\mathrm{d}}$. Phase curve $B_{\mathrm{e}}(\phi)$ is complex and was approximated here by a double wave. More homogeneous $B_{\mathrm{e}}$ points are necessary for the unique determination of the phase curve. Open circles - Ref. 1, filled circles - Refs. 1, 19, 208, 209 (phot. method); open squares - Ref. 270 (H lines), open circles - Ref. 327.

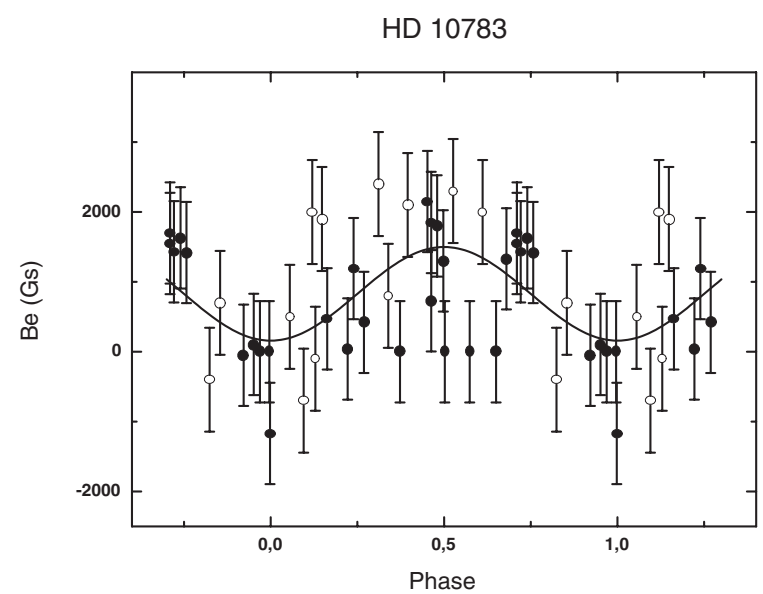

Fig. A.8. HD 10783. We determined $P_{\text {mag }}=4 \cdot 14628^{\mathrm{d}}$. It is close to the period $P_{\mathrm{mag}}=4.1565^{\mathrm{d}}$ taken from van Genderen (1964). Open circles - Ref. 1, filled circles - Ref. 35.

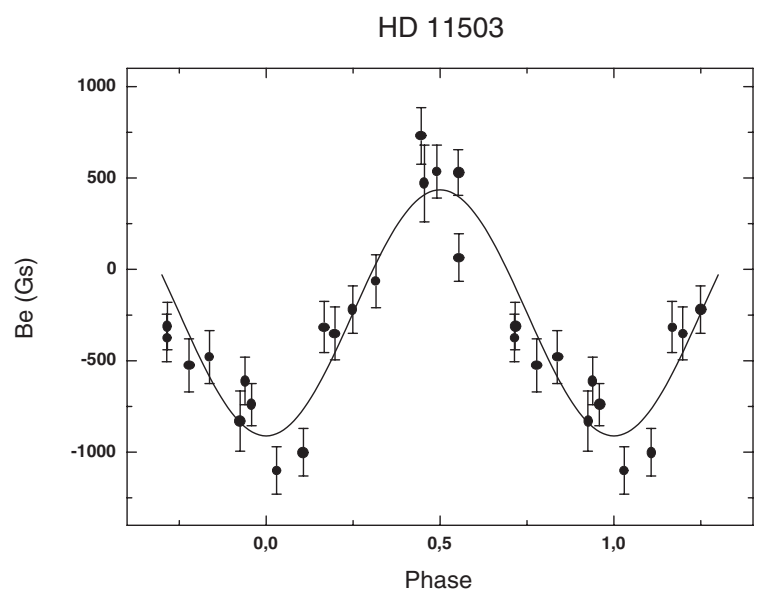

Fig. A.9. HD 11503. We used the period $P=1.190811^{\mathrm{d}}$ taken from Borra \& Landstreet (1980). We plotted here $B_{\mathrm{e}}$ observations from Ref. 2 only. $B_{\mathrm{e}}$ observations from Ref. 25 were not used, because apparently they are of very low accuracy.

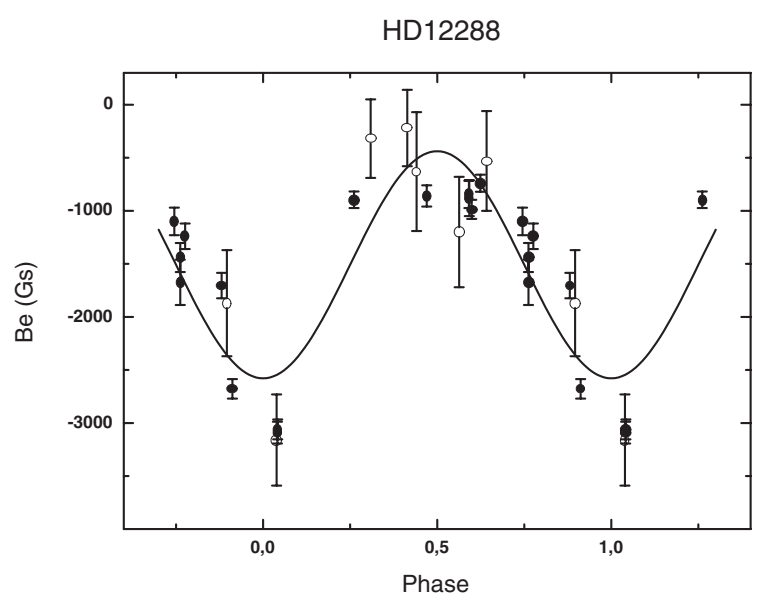

Fig. A.10. HD 12288. The period $P_{\mathrm{mag}}=34.79^{\mathrm{d}}$ of Ref. 312 was used. Open circles $-B_{\mathrm{e}}$ measurements from $\mathrm{H}$ lines, filled circles $-B_{\mathrm{e}}$ from metal lines.

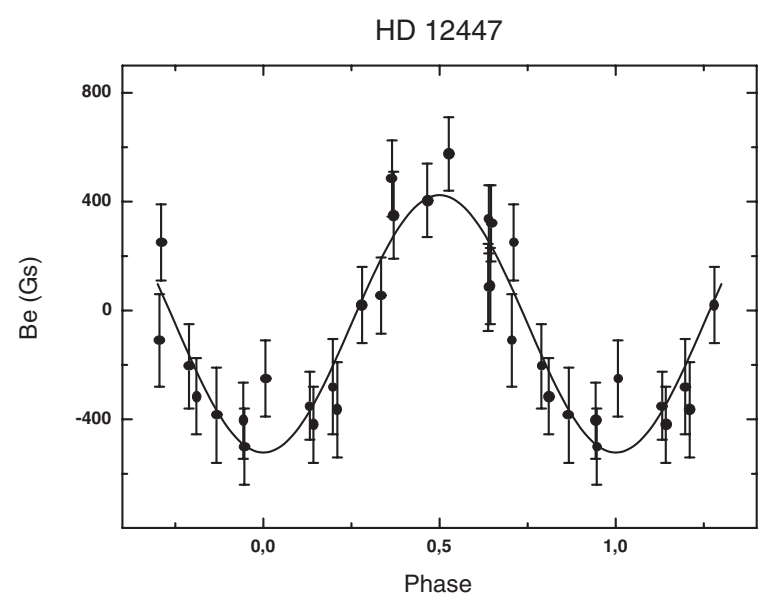

Fig. A.11. HD 12447. We used the period $P_{\text {mag }}=1.49070^{d}$ for plotting $B_{\mathrm{e}}$ data, which were taken from Ref. 2. Data from Ref. 25 were not used because they are of very low accuracy.

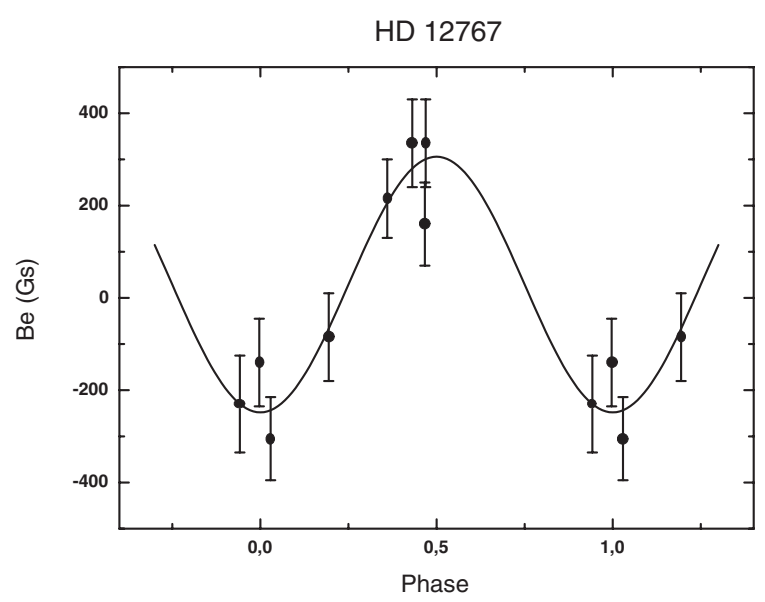

Fig. A.12. HD 12767. We used the period $P=1.892^{\mathrm{d}}$ taken from Ref. 137. Phase curve $B_{\mathrm{e}}(\phi)$ is derived from sparse data, and requires additional observations in phases $0.5-0.9$. 
V. D. Bychkov et al.: A catalog of stellar magnetic rotational phase curves, Online Material p 17

HD 14437

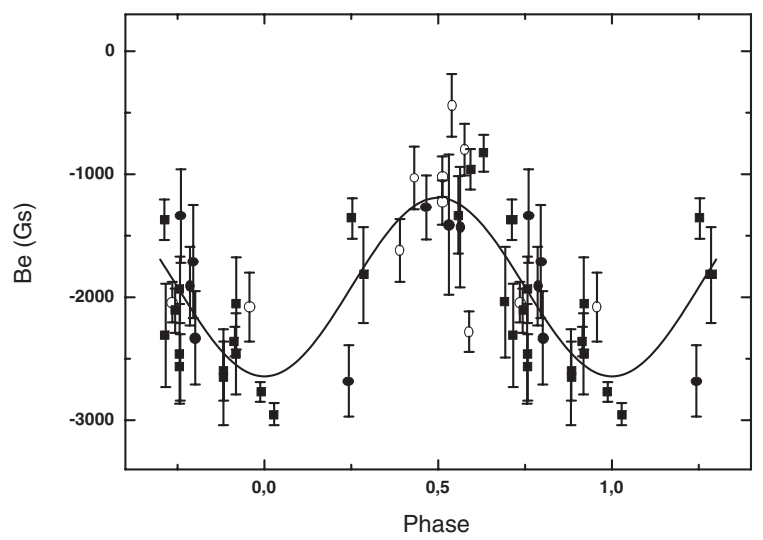

Fig. A.13. HD 14437. Open circles $-B_{\mathrm{e}}$ from Ref. 312 (phot. method), filled circles - data from $\mathrm{H}$ lines, Ref. 312, filled squares - metal lines \& CCD, Ref. 312. Additional $B_{\mathrm{e}}$ observations are necessary, because $P_{\text {mag }}$ is not well determined. Our best period is $P_{\text {mag }}=13.37^{\mathrm{d}}$.

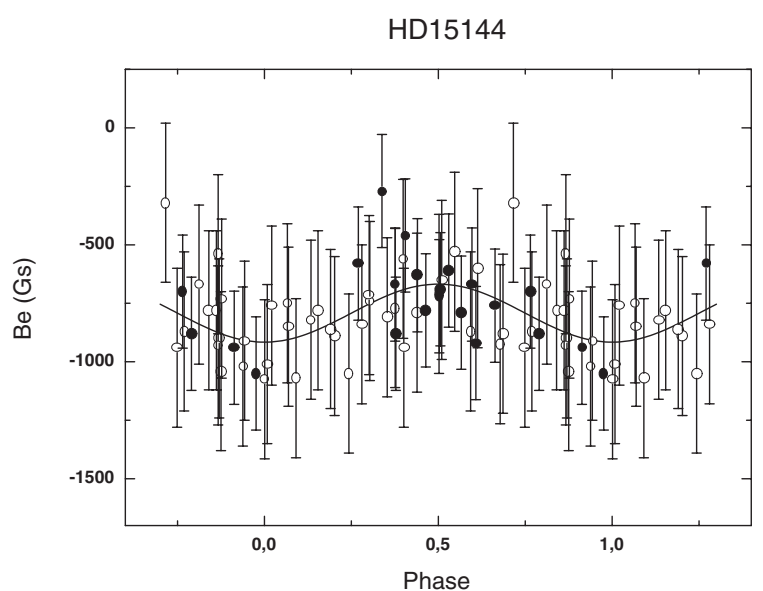

Fig. A.14. HD 15144. New high precision $B_{\mathrm{e}}$ data are necessary, since the rotational period $P_{\text {rot }}=2.9978724^{\mathrm{d}}$ taken from van Genderen (1971) differs from the magnetic period $P_{\mathrm{mag}}=15.88^{\mathrm{d}}$ (Bonsack 1981b). Open circles - phot. method, Ref. 1, filled circles - Ref. 87.

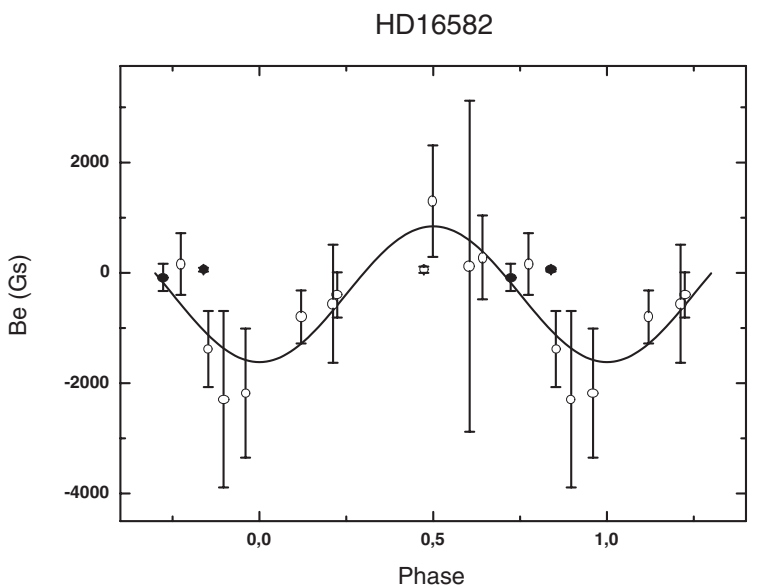

Fig. A.15. HD $16582=\delta$ Cet is a pulsating variable star of the $\beta C e p$ class, with the pulsation period $P_{\text {puls }}=0.16113668^{\mathrm{d}}$ (Mohan 1981). We determined the magnetic period $P_{\text {mag }}=1.40744^{\mathrm{d}}$, which perhaps can be identified with the rotational period of this star. Open circles phot. method, Refs. 77, 96; filled circles - Ref. 327.

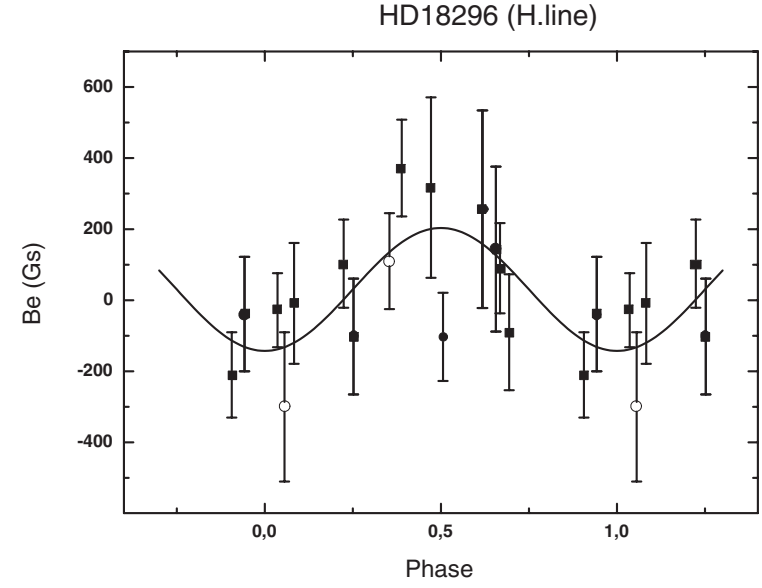

Fig. A.16. HD 18296. We used the period $P_{\mathrm{mag}}=2.88422^{\mathrm{d}}$ after Preston (1969b). $B_{\text {e }}$ points were measured in hydrogen lines. Open circles - Ref. 2, filled circles - Ref. 197, filled squares - Ref. 243.

HD18296 (Met.)

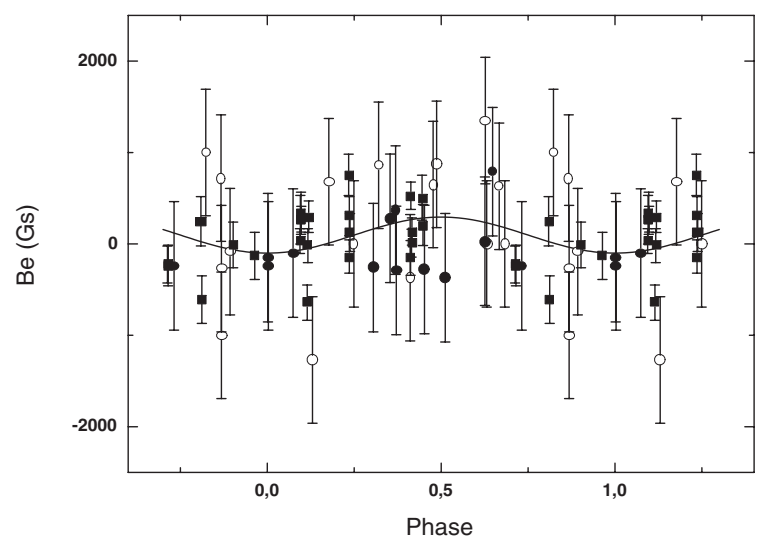

Fig. A.17. HD 18296. $B_{\mathrm{e}}$ measurements were obtained from metal lines. Open circles - Ref. 1, filled circles - Ref. 14, filled squares Ref. 327. We used the period $P_{\mathrm{mag}}=2.88422^{\mathrm{d}}$ after Preston (1969b).

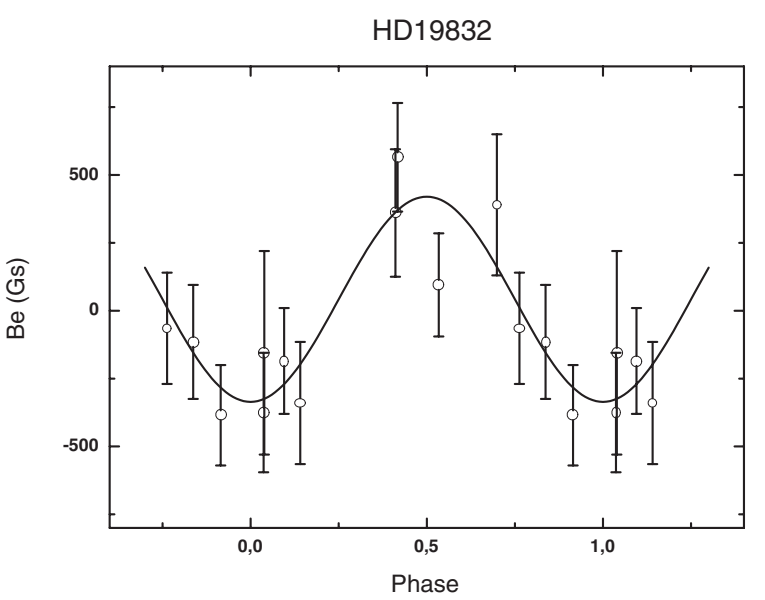

Fig. A.18. HD 19832. We used $B_{\mathrm{e}}$ estimates from Ref. 2, and the period $P_{\mathrm{mag}}=0.727893^{\mathrm{d}}$ taken from Stępień \& Czechowski (1993). 
V. D. Bychkov et al.: A catalog of stellar magnetic rotational phase curves, Online Material p 18

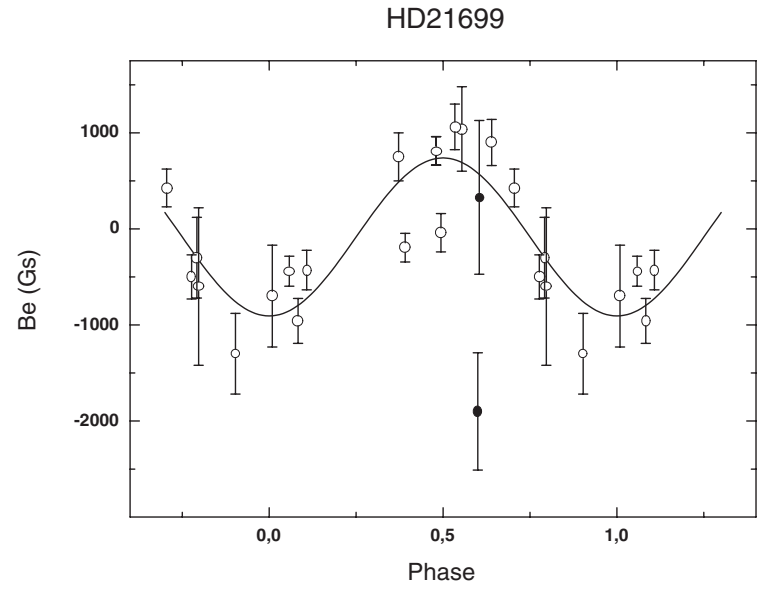

Fig. A.19. HD 21699. Magnetic $B_{\mathrm{e}}$ curve is fitted only to $\mathrm{H}$ line measurements, Ref. 252. We did not use low precision $B_{\mathrm{e}}$ points from metal lines, Ref. 40. Open circles - Ref. 252, filled circles - Ref. 40. We used the period $P_{\text {mag }}=2.4765^{\mathrm{d}}$ taken from Brown et al. (1985).

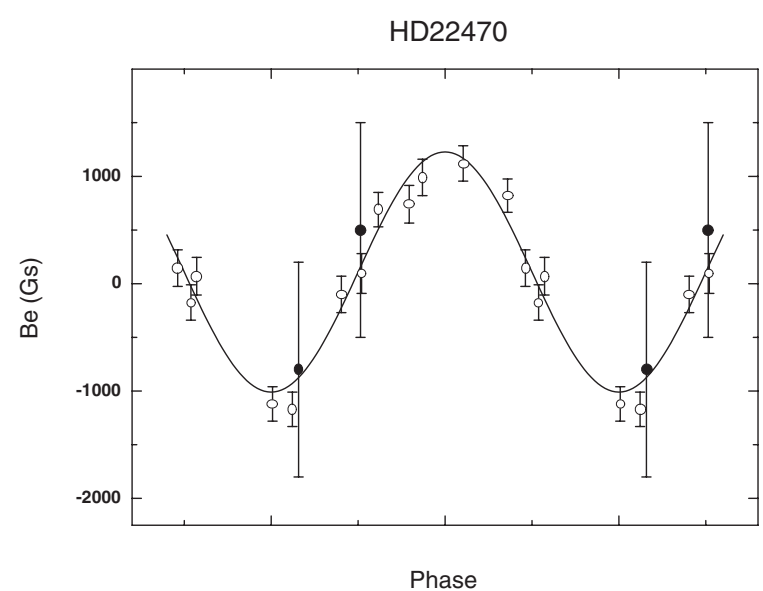

Fig. A.20. HD 22470. We used the period $P_{\mathrm{mag}}=0.6785^{\mathrm{d}}$ taken from Borra et al. (1983), Ref. 37. Open circles - Ref. 37, filled circles Ref. 25. This star needs additional observations for the unique period determination. Actually the value of $P_{\mathrm{mag}}=1.9387^{\mathrm{d}}$, taken from Adelman \& Boyce (1995) is still very tentative.

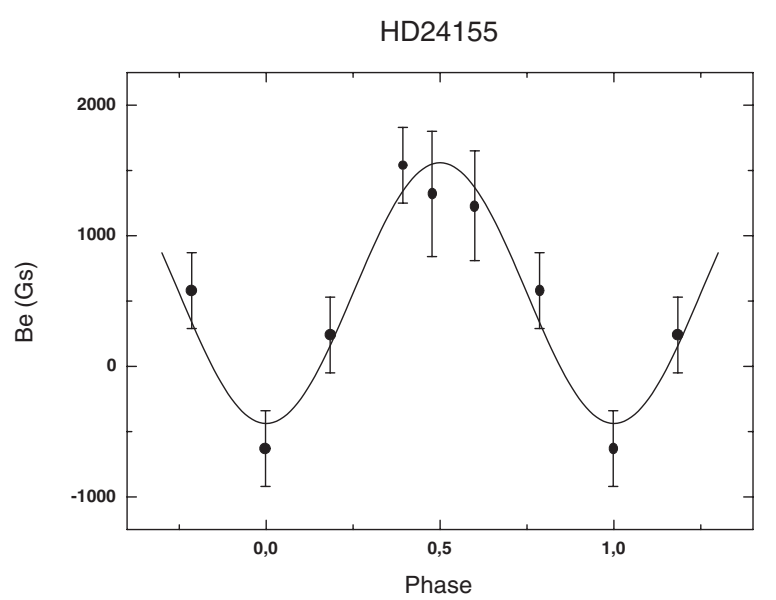

Fig. A.21. HD 24155. We used the period $P_{\text {mag }}=2.53465^{\mathrm{d}}$, taken from Ref. 230. This star requires additional observations, because the number of points is small. The actual $B_{\mathrm{e}}$ phase curve has been fitted to 6 points only.

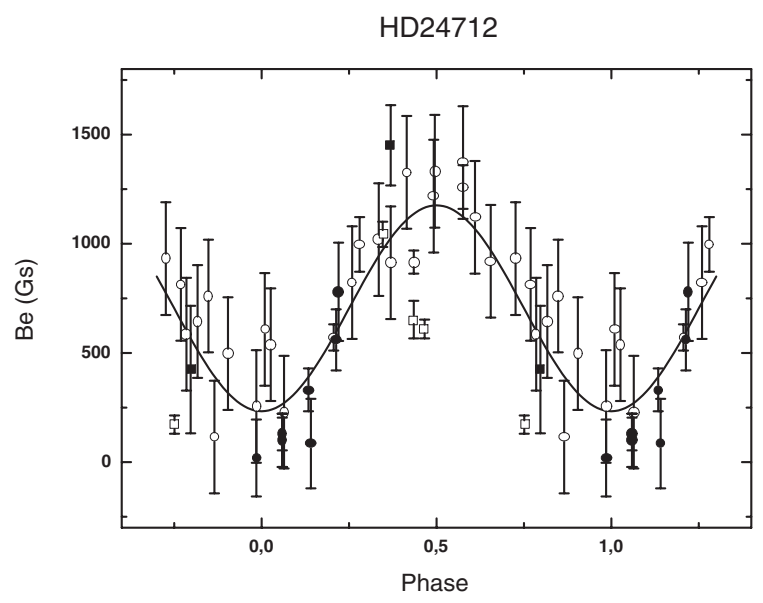

Fig. A.22. HD 24712. Our period $P_{\mathrm{mag}}=12.4617^{\mathrm{d}}$ fits well $B_{\mathrm{e}}$ measurements, and it is located inside the error box of the period $P_{\mathrm{mag}}=$ $12.4610 \pm 0.0011^{\mathrm{d}}$ by Bagnulo et al. (1995). Open circles - Refs. 21, 111, 120 (metal lines, phot. measurements); filled circles - Refs. 184, 256 (metal lines), open squares - Ref. 310 (LSD method); filled squares - Refs. 195, 327 (H line).

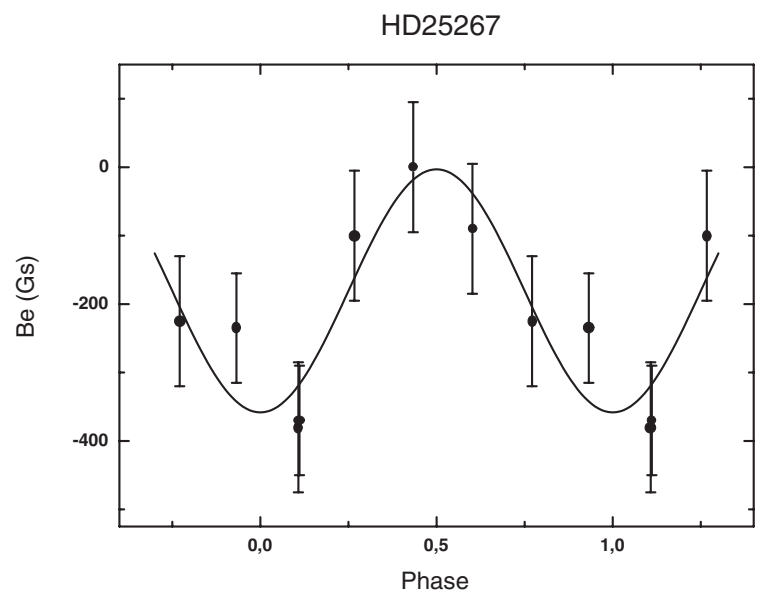

Fig. A.23. HD 25267. Period $P_{\mathrm{mag}}=5.954^{\mathrm{d}}$ was adapted from Ref. 2 . This star requires additional $B_{\mathrm{e}}$ observations for more exact period and magnetic curve determination.

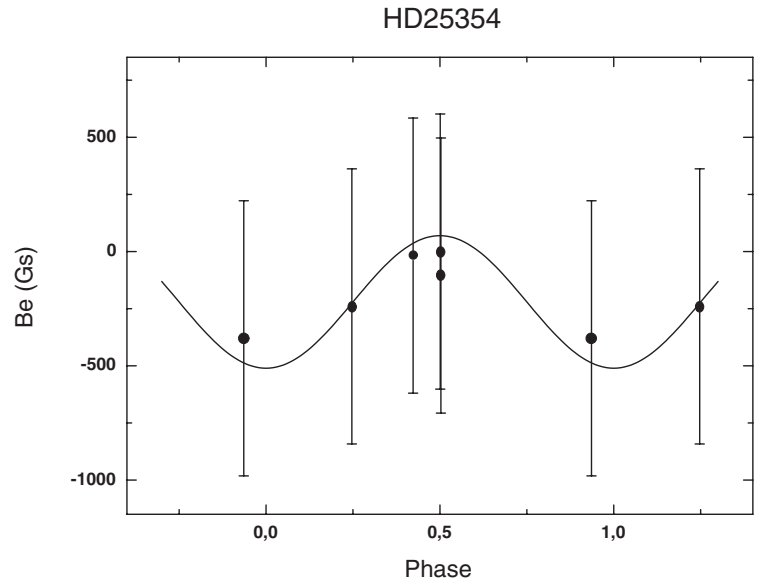

Fig. A.24. HD 25354. We used the period $P_{\mathrm{mag}}=3.90072^{\mathrm{d}}$, taken from Schoneich et al. (1976). Magnetic phase curve was derived from $5 B_{\mathrm{e}}$ points only. Additional $B_{\mathrm{e}}$ observations are necessary. 
V. D. Bychkov et al.: A catalog of stellar magnetic rotational phase curves, Online Material p 19

HD25823

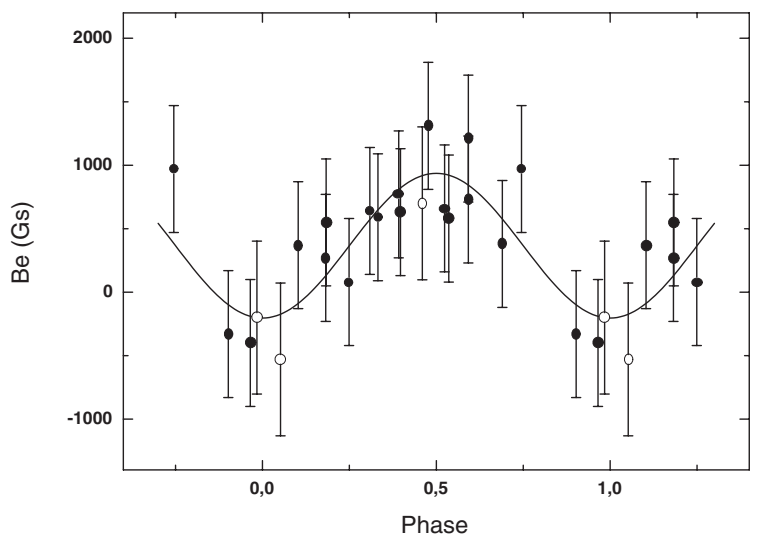

Fig. A.25. HD 25823. We used our magnetic period $P_{\mathrm{mag}}=4.65853^{\mathrm{d}}$. This period is shorter than the photometric period $P_{\text {phot }}=7.227424^{\mathrm{d}}$ (it is either rotational or orbital period) from Musielok \& Madej (1988). Open circles - Ref. 1, filled circles - Ref. 71.

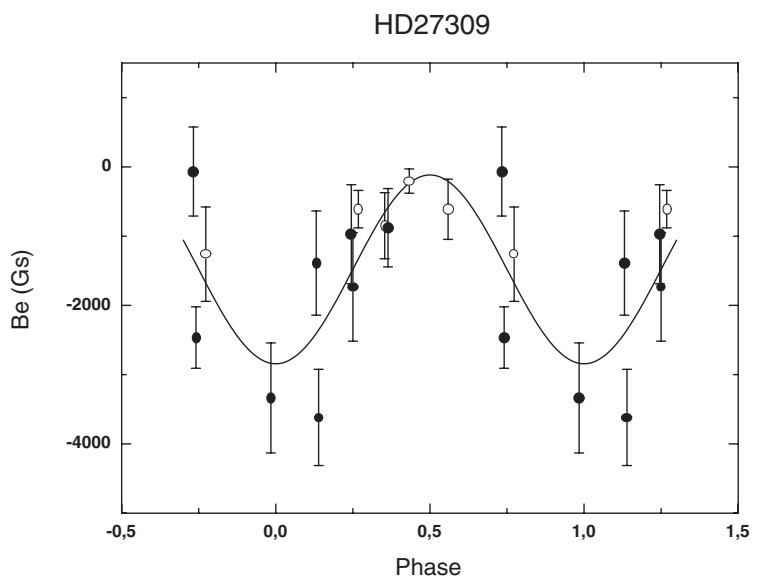

Fig. A.26. HD 27309. We used our magnetic period $P_{\mathrm{mag}}=1.10496^{\mathrm{d}}$. Photometric period $P_{\text {phot }}=1.5688840^{\mathrm{d}}$ from North \& Adelman (1995) apparently does not correspond to the magnetic observations. Additional $B_{\mathrm{e}}$ observations are necessary for the improvement of $P_{\text {mag. }}$. Open circles - Refs. 2, 41 (H lines); filled circles - Ref. 142 (metal lines).

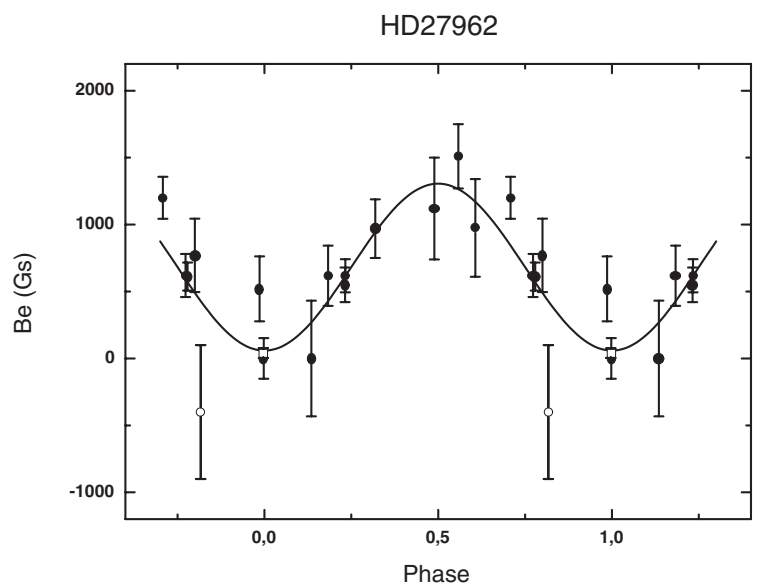

Fig. A.27. HD 27962. No credible photometric period is available. We determined the most probable magnetic period, $P_{\mathrm{mag}}=2.13^{\mathrm{d}}$. Open circles - Ref. 1, filled circles - Ref. 98, open squares - Ref. 77.

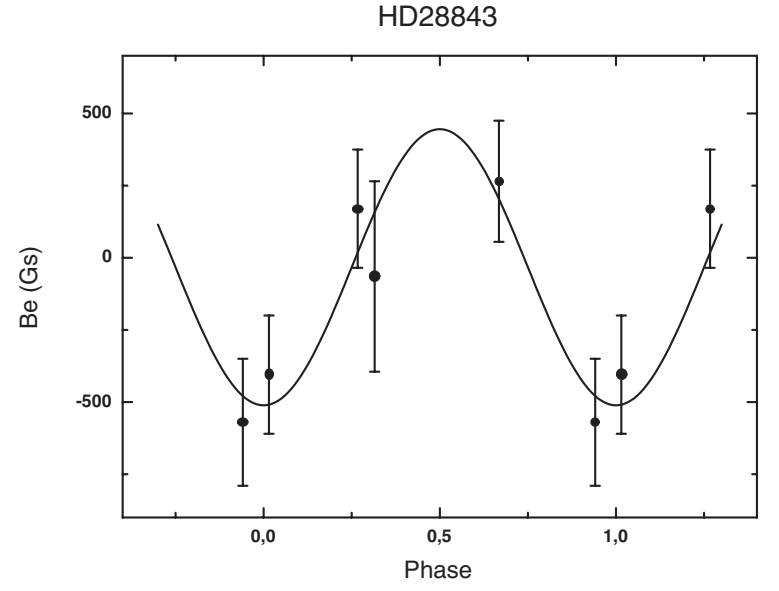

Fig. A.28. HD 28843. We used the period $P_{\text {mag }}=1.373813^{\mathrm{d}}$ taken from Mathys et al. (1986). Magnetic rotational phase curve was formally derived from 5 points only. New $B_{\mathrm{e}}$ observations are necessary.

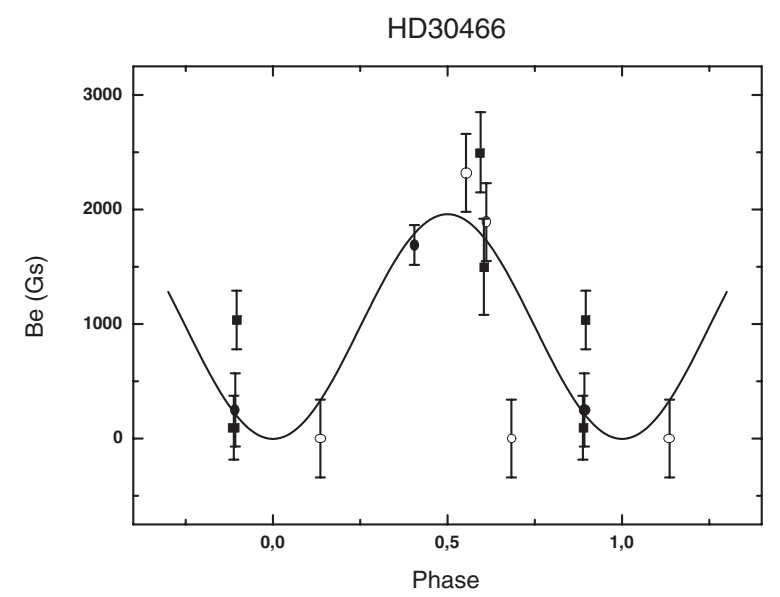

Fig. A.29. HD 30466. The most probable magnetic period $P_{\text {mag }}=1.3900^{\mathrm{d}}$ was taken from Rakosch \& Fiedler (1978). The number of $B_{\mathrm{e}}$ points is low. New high precision measurements are necessary. Open circles - Ref. 1, filled circles - Ref. 142, open squares - Ref. 327 . 
V. D. Bychkov et al.: A catalog of stellar magnetic rotational phase curves, Online Material p 20

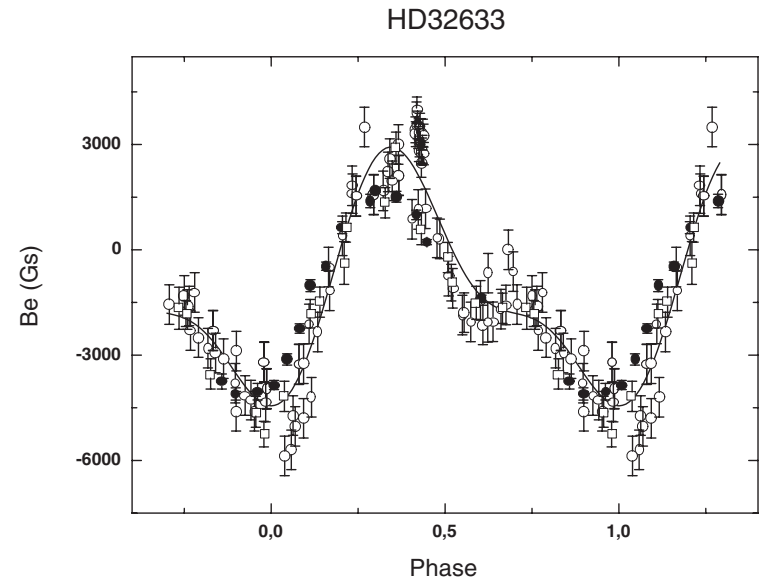

Fig. A.30. HD 32633. We used here the period $P=6.4300^{\mathrm{d}}$, taken from Renson (1972), and obtained complex double wave magnetic rotational phase curve. This figure displays all the available $B_{\mathrm{e}}$ data. Open circles - Refs. 1, 8, 175, 327 (phot. method), filled circles Refs. 184, 310 (LSD), open squares - Ref. 2 (H lines). Phase curves obtained by various methods significantly differ, see two following figures.

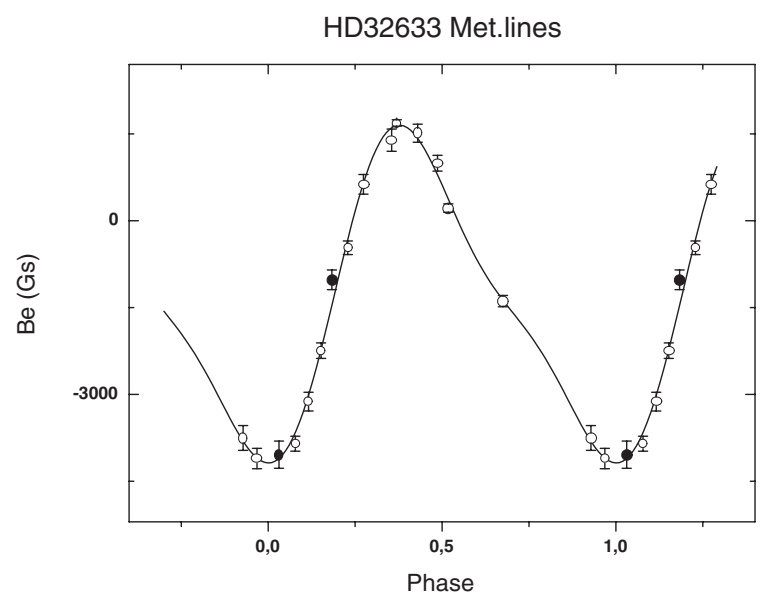

Fig. A.31. HD 32633. We applied the period $P=6.4300^{\mathrm{d}}$, taken from Renson (1972). This phase curve was obtained from high accuracy LSD measurements. There exists only one $B_{\mathrm{e}}$ point around $\phi \approx 0.5$, and the new data would be very important to determine the phase curve. Open circles - Ref. 310 (LSD method), filled circles - Ref. 184.

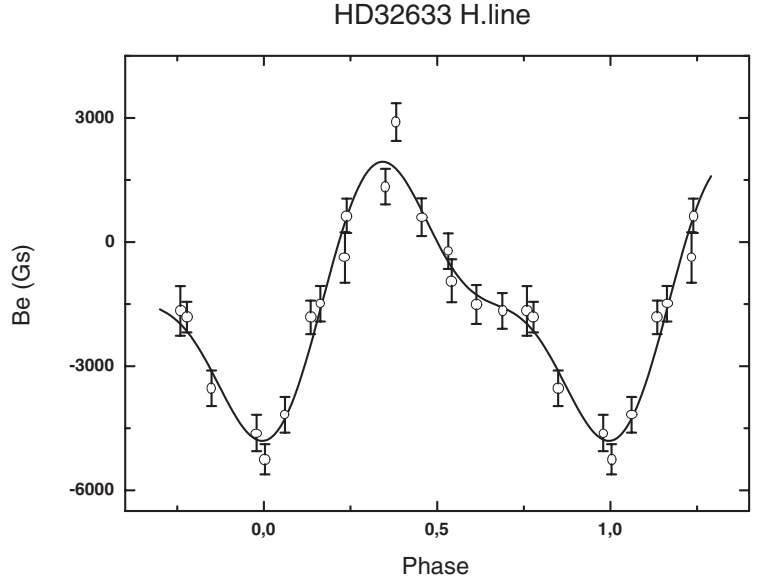

Fig. A.32. HD 32633. We used the period $P_{\text {mag }}=6.4300^{\mathrm{d}}$, taken from Renson (1972). This is the magnetic rotational phase curve derived from $B_{\mathrm{e}}$ points measured in $\mathrm{H}$ lines - Ref. 2.

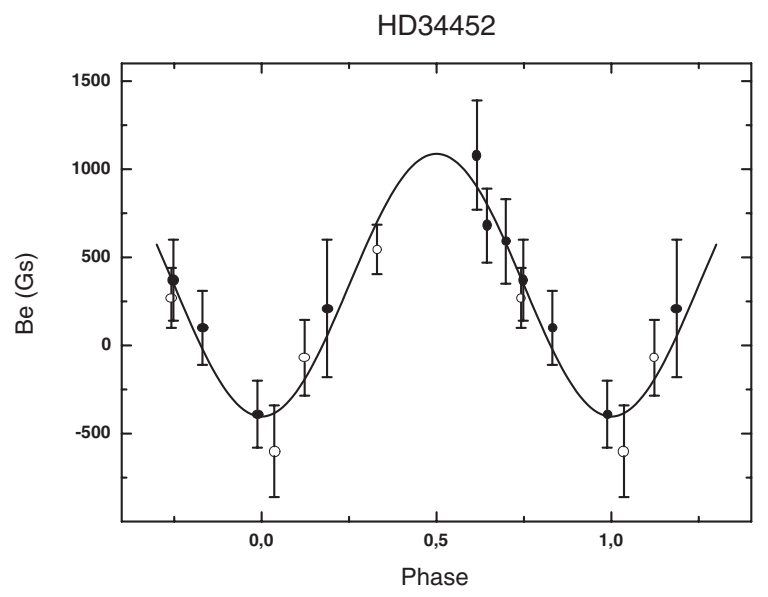

Fig. A.33. HD 34452. Bohlender et al. (1993), Ref. 230, determined the period $P_{\mathrm{mag}}=2.466264^{\mathrm{d}}$. However, we propose that the best period is $P_{\mathrm{mag}}=2.4687^{\mathrm{d}}$, and used it to plot this figure. The rotational phase curve does not include photographic $B_{\mathrm{e}}$ observations from Ref. 41. Open circles - Ref. 2, filled circles - Ref. 230.

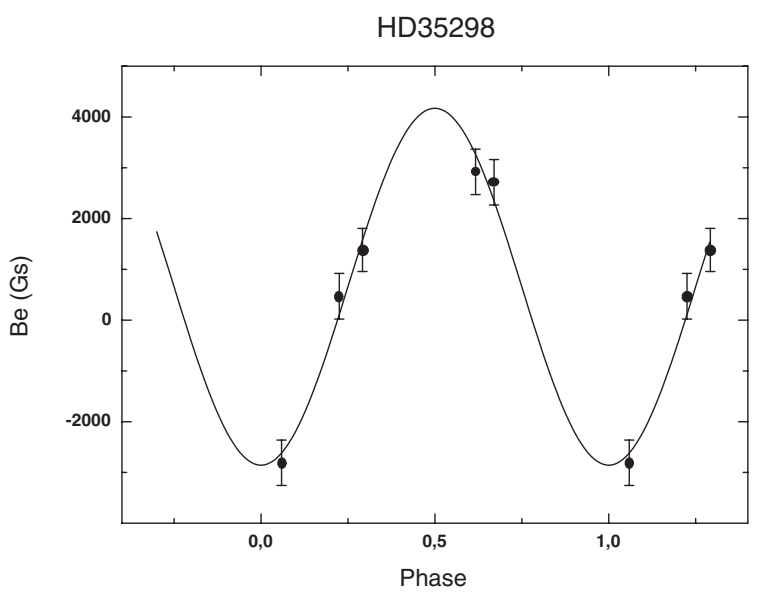

Fig. A.34. HD 35298. Good photometric period $P_{\text {phot }}=1.85336^{\mathrm{d}}$ was taken from North (1984). Number of $B_{\mathrm{e}}$ observations is rather small. Magnetic field $B_{\mathrm{e}}$ (Ref. 201) is strong and is highly variable with the rotational phase, but the accurate phase curve determination requires more $B_{\mathrm{e}}$ observations. 
V. D. Bychkov et al.: A catalog of stellar magnetic rotational phase curves, Online Material p 21

HD35456

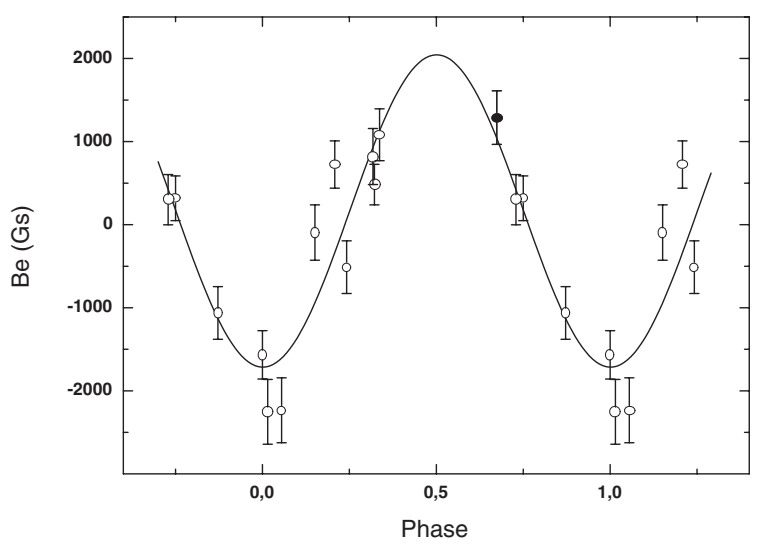

Fig. A.35. HD 35456. The best our magnetic period $P_{\text {mag }}=0.28313^{\mathrm{d}}$. Such an ultrashort period results from $B_{\mathrm{e}}$ data in Ref. 201. (In Ref. 201 one can see that $B_{\mathrm{e}}$ changed by $3074 \mathrm{G}$ in 0.086 days.) More $B_{\mathrm{e}}$ observations are necessary for the reliable $P_{\mathrm{mag}}$ and $B_{\mathrm{e}}(\phi)$ determinations. Open circles - Ref. 201 (H line), filled circles - Ref. 327 (phot. method).

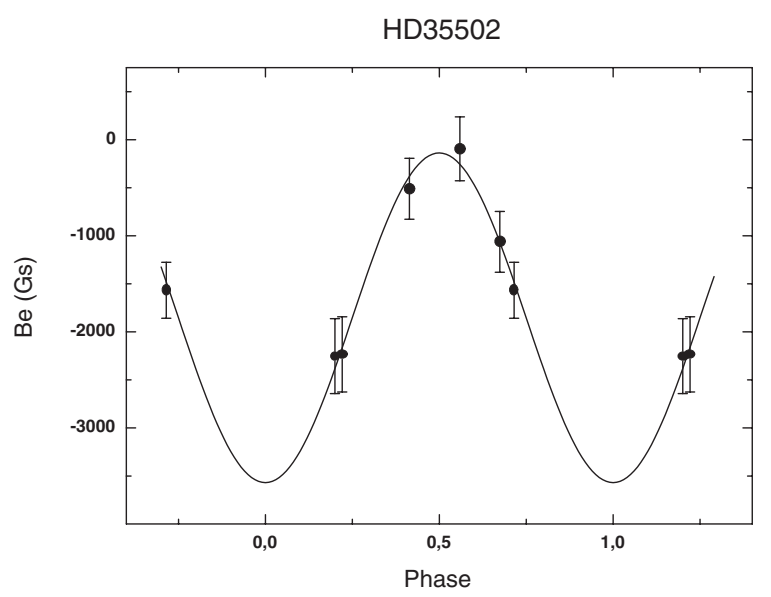

Fig. A.36. HD 35502. We used the period $P=1.707^{\mathrm{d}}$, taken from Borra (1981). More $B_{\mathrm{e}}$ observations are necessary to obtain a complete phase curve. H line $B_{\mathrm{e}}$ points are from Ref. 201.

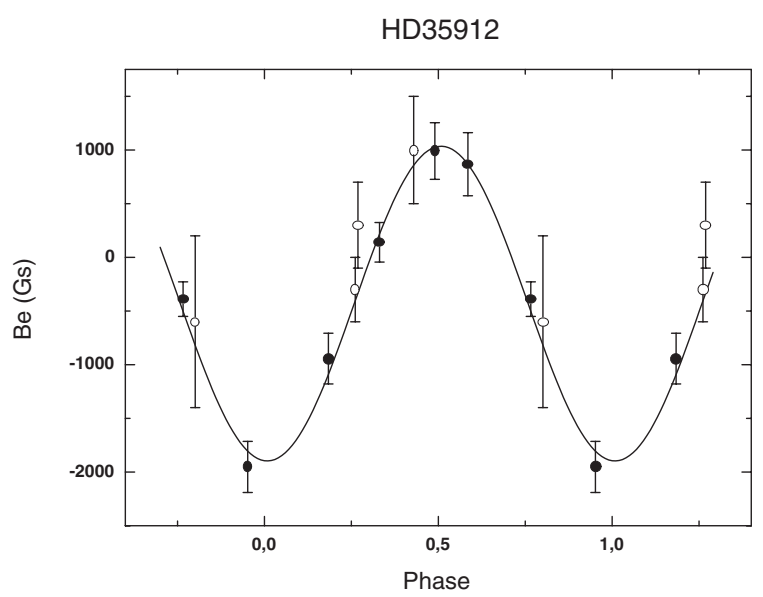

Fig. A.37. HD 35912. We have determined the probable period of magnetic variability, $P_{\mathrm{mag}}=0.89786^{\mathrm{d}}$, using the available observations of $B_{\mathrm{e}}$. New magnetic observations are necessary to improve the phase curve. Open circles - Ref. 55, filled circles - Ref. 327.

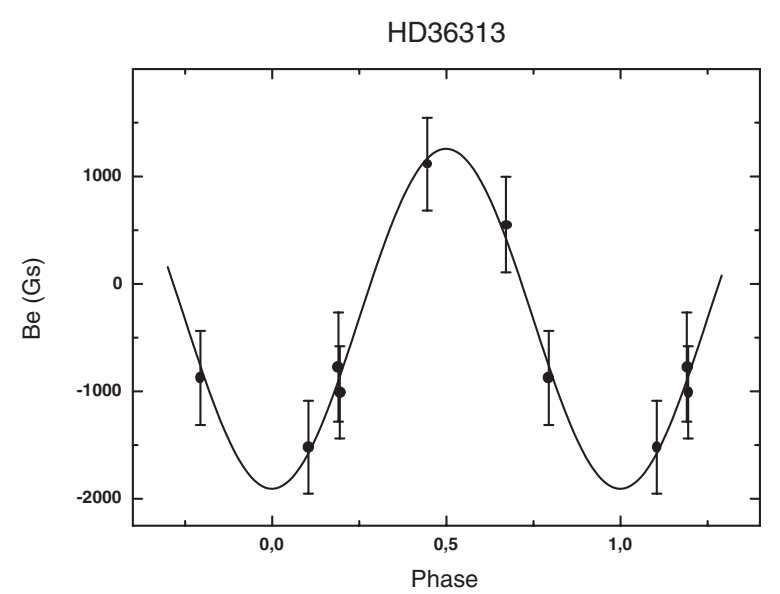

Fig. A.38. HD 36313. We determined the best magnetic period $P_{\text {mag }}=0.77677^{\mathrm{d}}$. Phase curve $B_{\mathrm{e}}(\phi)$ should be improved by additional $B_{\mathrm{e}}$ points. Magnetic observations were taken from Ref. 201 (H lines).

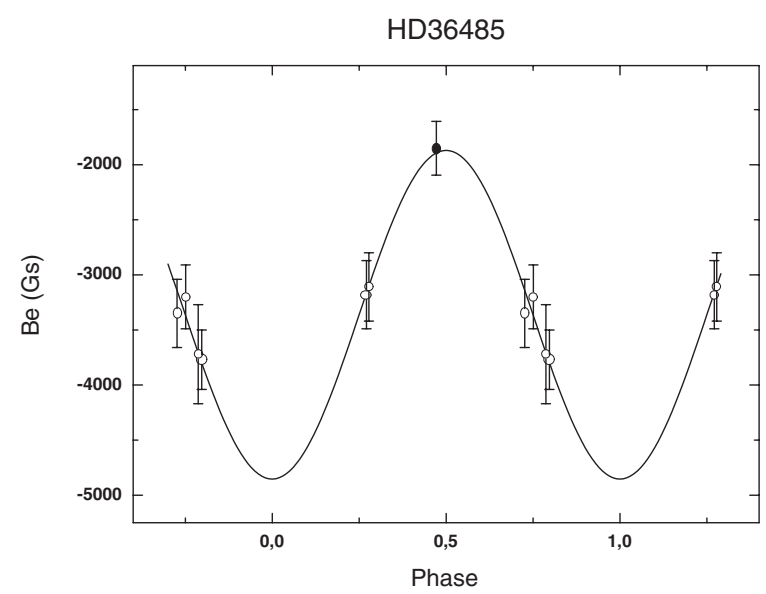

Fig. A.39. HD 36485. We determined the probable period of the magnetic variability, $P_{\text {mag }}=2.0129^{\mathrm{d}}$. Additional $B_{\mathrm{e}}$ measurements are required to improve the phase curve $B_{\mathrm{e}}(\phi)$. Values of $B_{\mathrm{e}}$ obtained by different methods probably exhibit large systematic differences. Open circles - Ref. 55 (H line), filled circles - Ref. 256 (met. lines).

HD36526

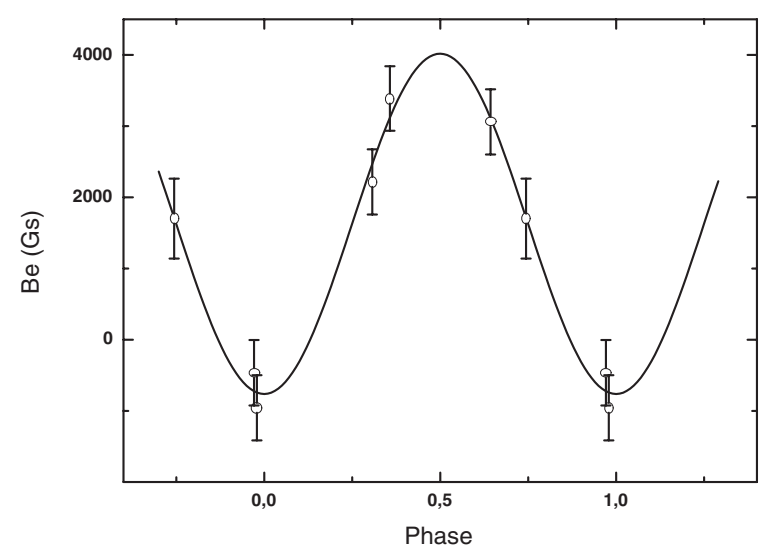

Fig. A.40. HD 36526. We used the period $P=1.5405^{\mathrm{d}}$, taken from North (1984). Magnetic field of the star is strong and its longitudinal component is highly variable with the rotational phase. More $B_{\mathrm{e}}$ observations are necessary to obtain a complete phase curve. 
V. D. Bychkov et al.: A catalog of stellar magnetic rotational phase curves, Online Material p 22

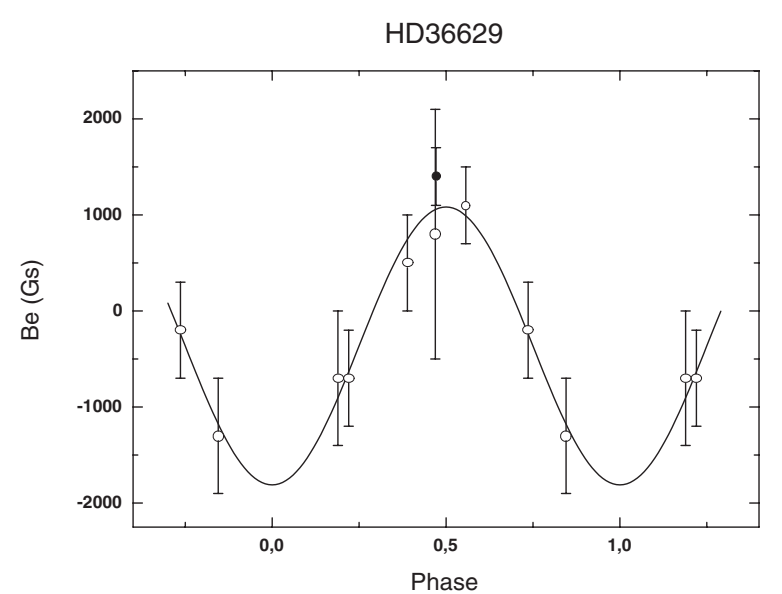

Fig. A.41. HD 36629. We determined the most probable magnetic period, $P_{\text {mag }}=5.01255^{\mathrm{d}}$. The number of available $B_{\mathrm{e}}$ observations is small and of low accuracy. Also here magnetic field is strong and its $B_{\mathrm{e}}(\phi)$ exhibits large amplitude. Additional $B_{\mathrm{e}}$ observations are necessary to improve the magnetic rotational phase curve. Open circles Ref. 55, filled circles - Ref. 53 (metal lines, and phot. method were used in both papers).

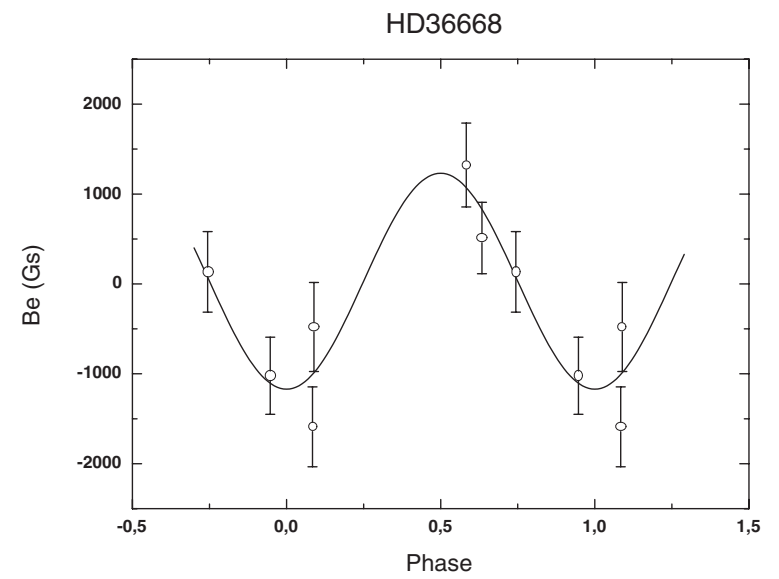

Fig. A.42. HD 36668. We used the period $P_{\text {mag }}=2.1211^{\mathrm{d}}$, taken from North (1984). The number of $B_{\mathrm{e}}$ observations is small. The magnetic field of this star is strong. We need more points to obtain a satisfactory $B_{\mathrm{e}}(\phi)$ phase curve.

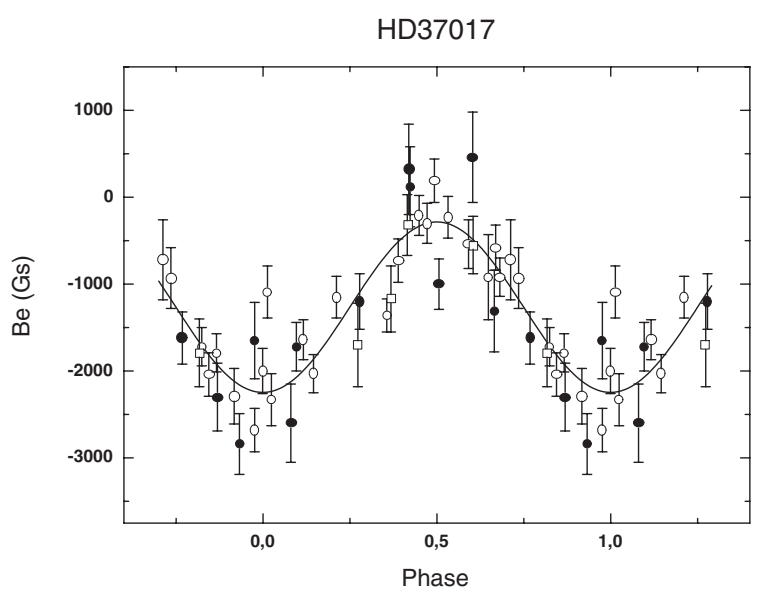

Fig. A.43. HD 37017. We used the period $P_{\mathrm{mag}}=0.901175^{\mathrm{d}}$, taken from Borra \& Landstreet (1979), Ref. 24. Open circles - Ref. 135 (H lines), filled circles - Ref. 24 (H lines), open squares - Ref. 135 (He 5867 line).

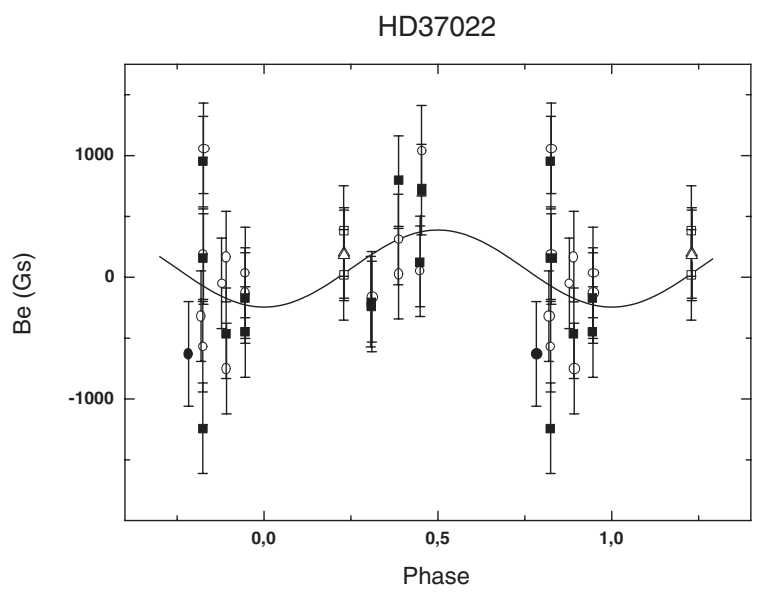

Fig. A.44. HD 37022. Ae/Be star with the rotation period $P_{\text {rot }}=15.422^{\mathrm{d}}$ taken from Stahl et al. (1996). Filled squares Ref. 286, open circles - Ref. 348 (absorption line CIV 5801), filled circles - Ref. 348 (abs. line CIV 5812), open squares - Ref. 348 (abs. line HeI 4713), open triangles - Ref. 348 (abs. line HeI 4541).

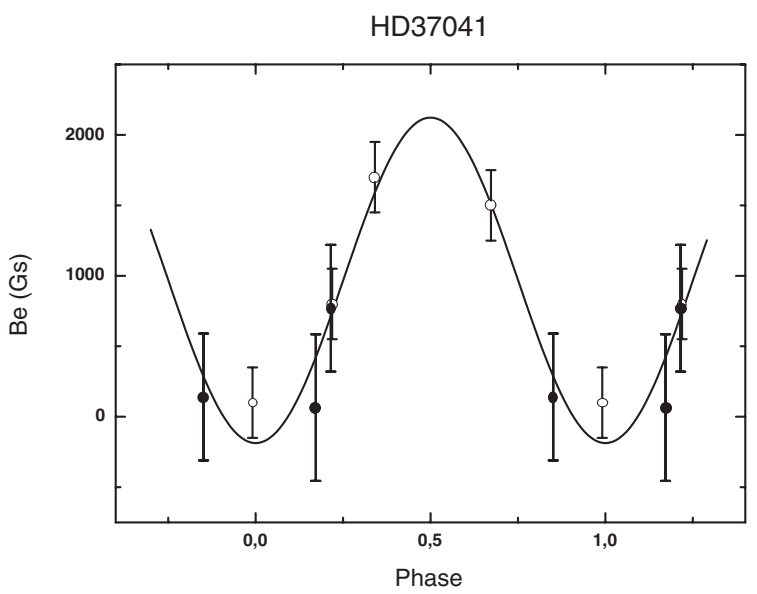

Fig. A.45. HD 37041. We determined the most probable period of magnetic variability, $P_{\mathrm{mag}}=3.14269^{\mathrm{d}}$. Only few $B_{\mathrm{e}}$ points are available, additional observations are necessary for the reliable $P_{\text {mag }}$ determination. Open circles - Ref. 69 (H lines), filled circles - Ref. 75 (He 6678 line). 
V. D. Bychkov et al.: A catalog of stellar magnetic rotational phase curves, Online Material p 23

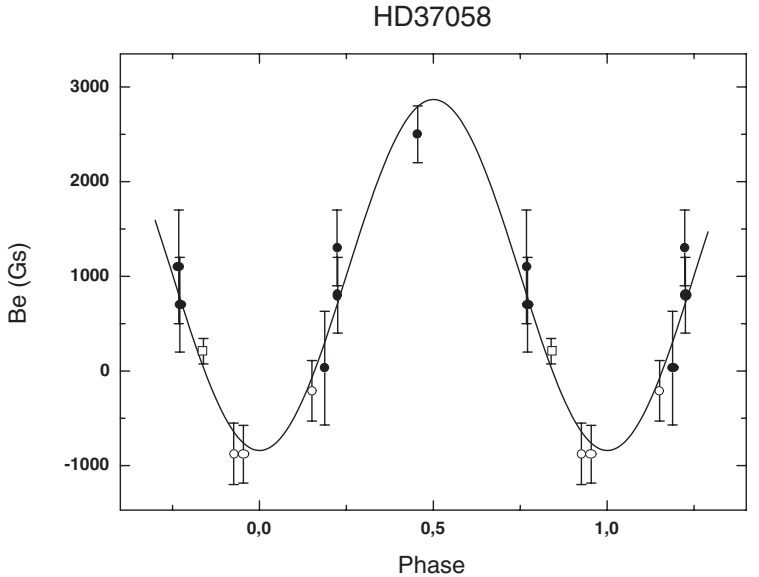

Fig. A.46. HD 37058. We determined and used here the best magnetic period $P_{\mathrm{mag}}=1.022^{\mathrm{d}}$, and this value disagrees with the period frequently quoted earlier, equal to $14.612^{\mathrm{d}}$, see Ref. 256. Magnetic points $B_{\mathrm{e}}$ are sparse. Open circles - Ref. 37 (H line), filled circles - Refs. 53, 55 (metal lines, phot. method), open squares - Ref. 256 (metal lines).

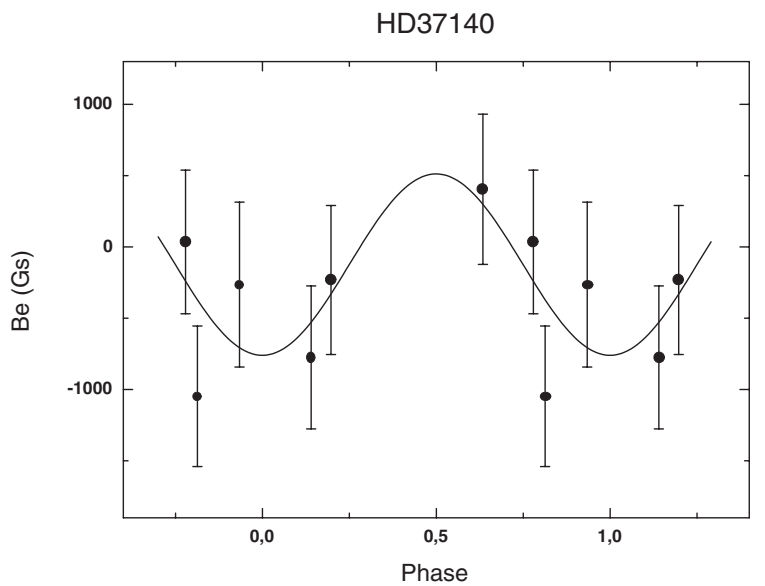

Fig. A.47. HD 37140. We used the period $P_{\mathrm{mag}}=2.7088^{\mathrm{d}}$, taken from North (1984). The number of existing $B_{\mathrm{e}}$ points is very small. We need more magnetic observations of this star. Open circles - Ref. 201 (H lines).

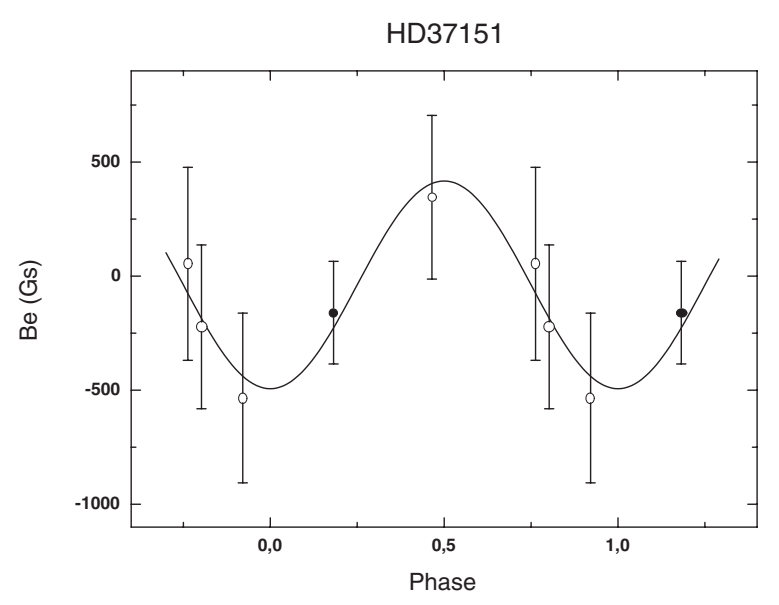

Fig. A.48. HD 37151. Photometric period $P_{\text {phot }}=5.6732^{\mathrm{d}}$ (North 1984) seems the best for the definition of the rotational magnetic phase curve. Number of available $B_{\mathrm{e}}$ points is very low, and new magnetic measurements are necessary. Open circles - Ref. 201 (H line), filled circles - Ref. 204 (H line).

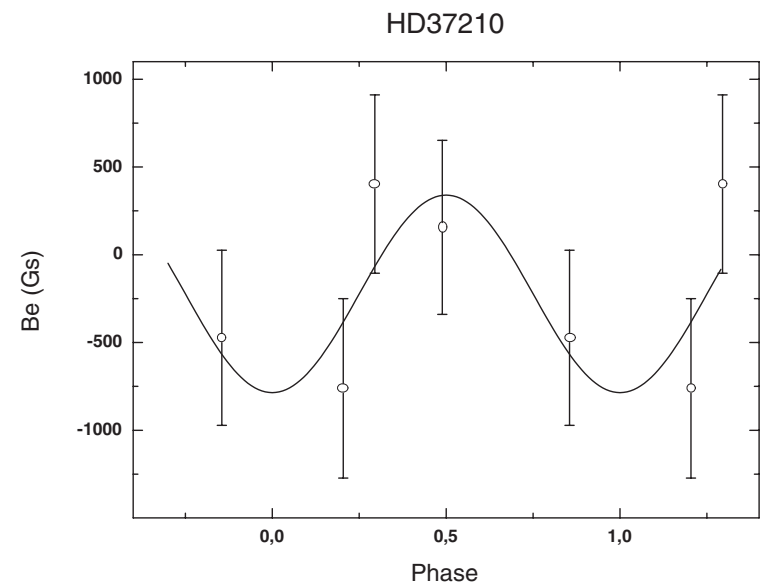

Fig. A.49. HD 37210. We used the photometric period $P_{\text {phot }}=11.0494^{\mathrm{d}}$, taken from North (1984). Only few $B_{\mathrm{e}}$ points are available, new magnetic observations are necessary to obtain better phase curve. Open circles - Ref. 201 (H lines).

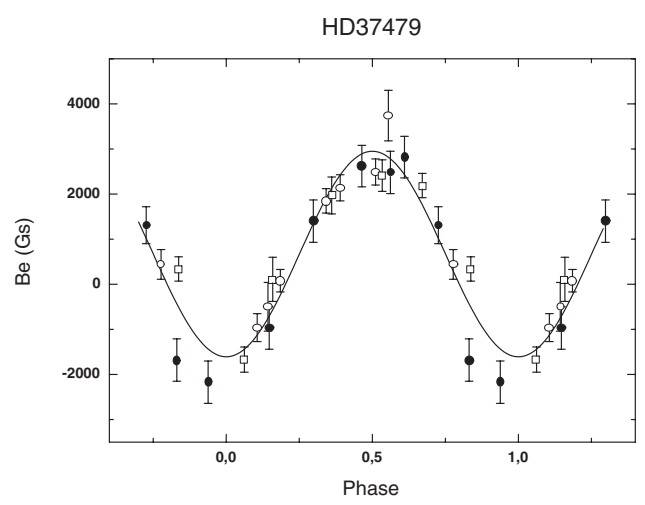

Fig. A.50. HD 37479. We used the period $P_{\mathrm{mag}}=1.190811^{\mathrm{d}}$, taken from Shore \& Brown (1990). Open circles - Ref. $28\left(B_{\mathrm{e}}\right.$ from $\mathrm{H}$ lines), filled circles - Ref. 135 (H lines), open squares - Ref. 135 (HeI 5876 line) 
V. D. Bychkov et al.: A catalog of stellar magnetic rotational phase curves, Online Material p 24

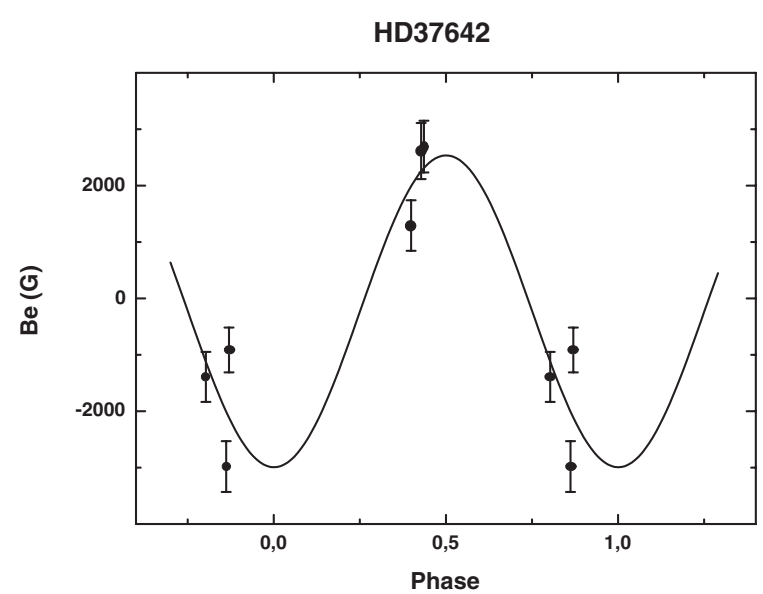

Fig. A.51. HD 37642. We used the period $P_{\mathrm{mag}}=1.07977^{\mathrm{d}}$, taken from North (1984). Very few $B_{\text {e }}$ points are available. Longitudinal magnetic field is strong and exhibits strong variability, but more observations are necessary to obtain the magnetic rotational phase curve and the $P_{\text {mag. }}$.

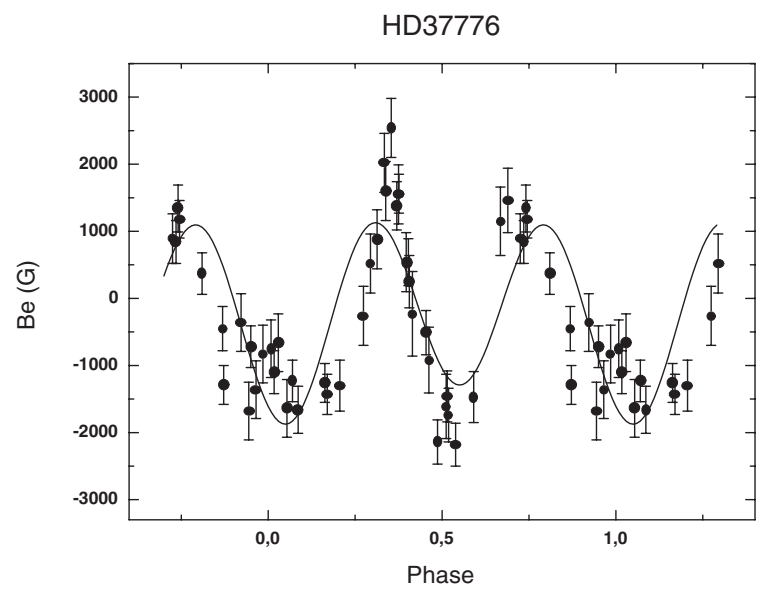

Fig. A.52. HD 37776. We used the period $P=1.538675^{\mathrm{d}}$, taken from Shore \& Brown (1990). The phase curve has a very complicated form. The averaged curve was constructed only with $B_{\mathrm{e}}$ points measured in $\mathrm{H}_{\beta}$ line. These measurements were taken from Ref. 174.

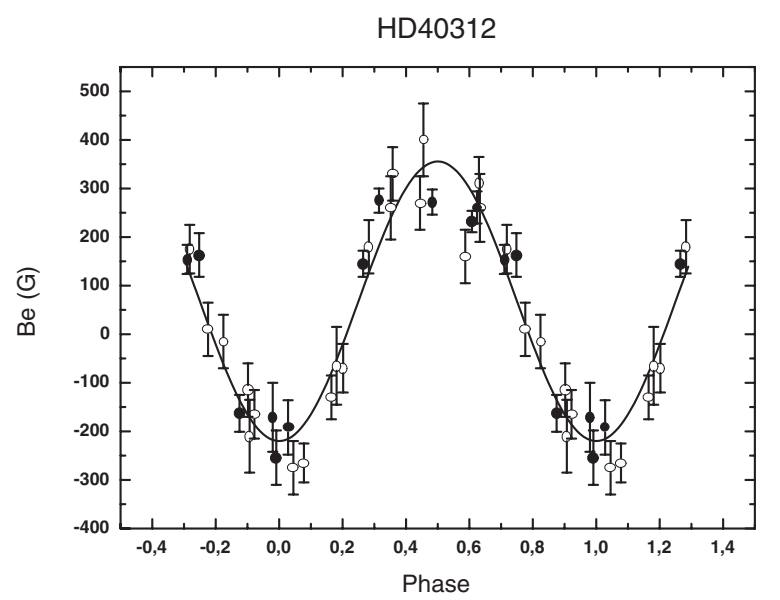

Fig. A.53. HD 40312. The above phase curve was computed with the improved magnetic period $P_{\mathrm{mag}}=3.61866^{\mathrm{d}}$. We determined $P_{\mathrm{mag}}$ from $B_{\mathrm{e}}$ measurements in Refs. 2 and 310. Open circles - Ref. 2 (H lines), filled circles - Ref. 310 (metal lines, LSD method).

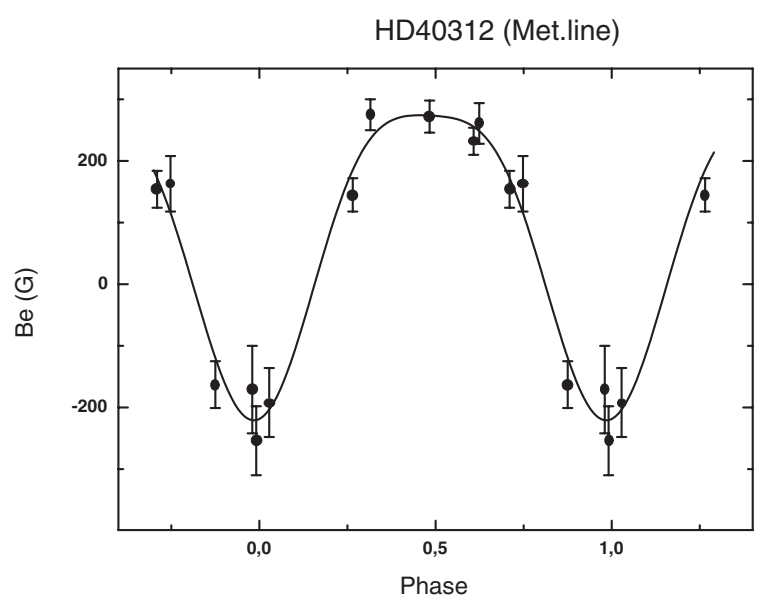

Fig. A.54. HD 40312. As above, we applied the period $P_{\text {mag }}=3.61866^{\mathrm{d}}$ determined for $B_{\mathrm{e}}$ points in Refs. 2 and 310. Magnetic data of high accuracy in Ref. 310 (metal lines) reveal deviations from sine wave near $\phi=0.5$. Therefore the $B_{\mathrm{e}}(\phi)$ phase curve was fitted here by a double wave. Filled circles - Ref. 310 (metal lines, LSD method).

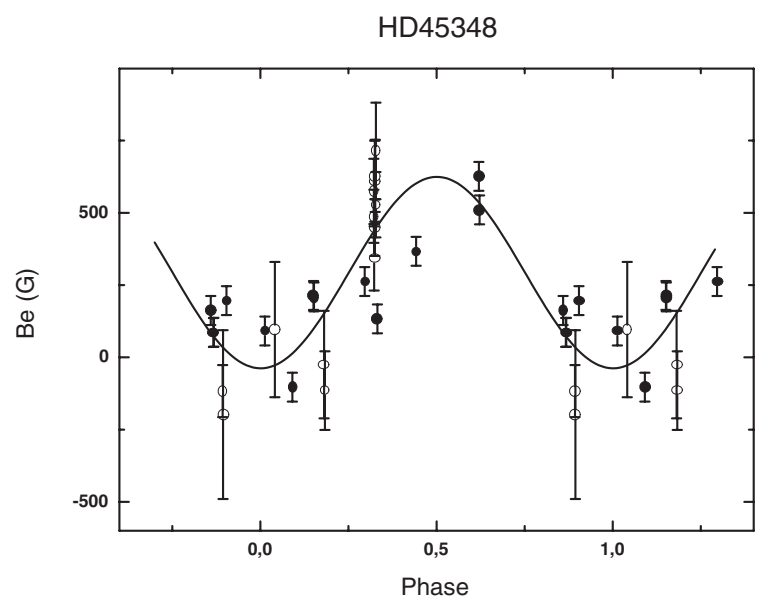

Fig. A.55. HD 45348. We used the period $P_{\mathrm{mag}}=6.90^{\mathrm{d}}$, taken from Weiss (1986). The amplitude of $B_{\mathrm{e}}$ variations is low. New magnetic observations of high accuracy are necessary here, in particular for the precise determination of the period $P_{\mathrm{mag}}$. Filled circles - Ref. 42, open circles - Ref. 273 (metal lines, phot. method). 
V. D. Bychkov et al.: A catalog of stellar magnetic rotational phase curves, Online Material p 25

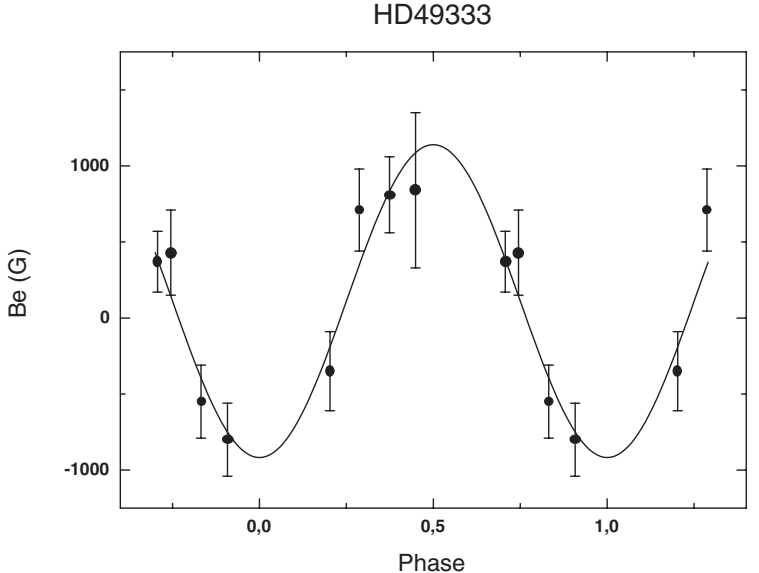

Fig. A.56. HD 49333. Magnetic field $B_{\mathrm{e}}$ was investigated in Refs. 37, and 230 from hydrogen lines. We used the period $P_{\text {mag }}=2.18010^{\mathrm{d}}$, taken from Bohlender et al. (1993), Ref. 230. Measurements are scarce, new additional $B_{\mathrm{e}}$ observations are necessary.

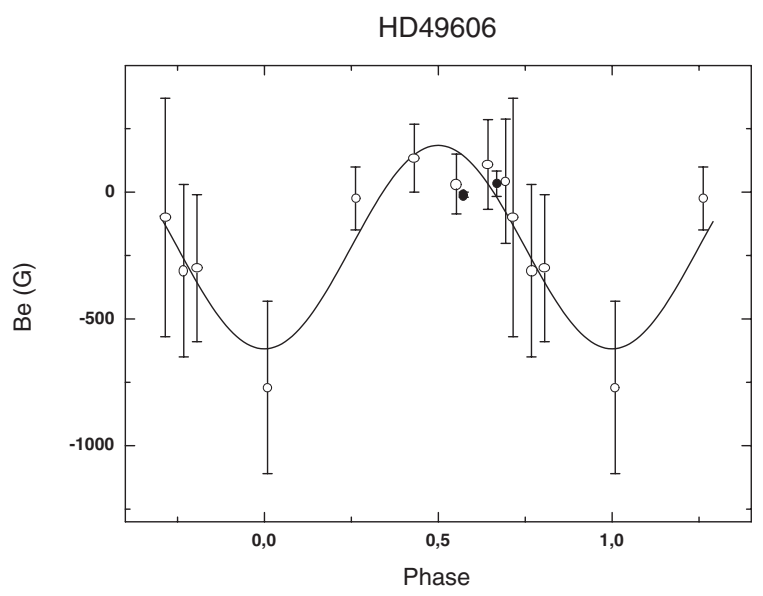

Fig. A.57. HD 49606. We determined the best magnetic period, $P_{\text {mag }}=1.10503^{\mathrm{d}}$. The available $B_{\mathrm{e}}$ points are scarce, and new observations are necessary to obtain more exact phase curve. Open circles - Refs. 230, 267 (H lines), filled circles - Ref. 330 (metal lines, LSD method).

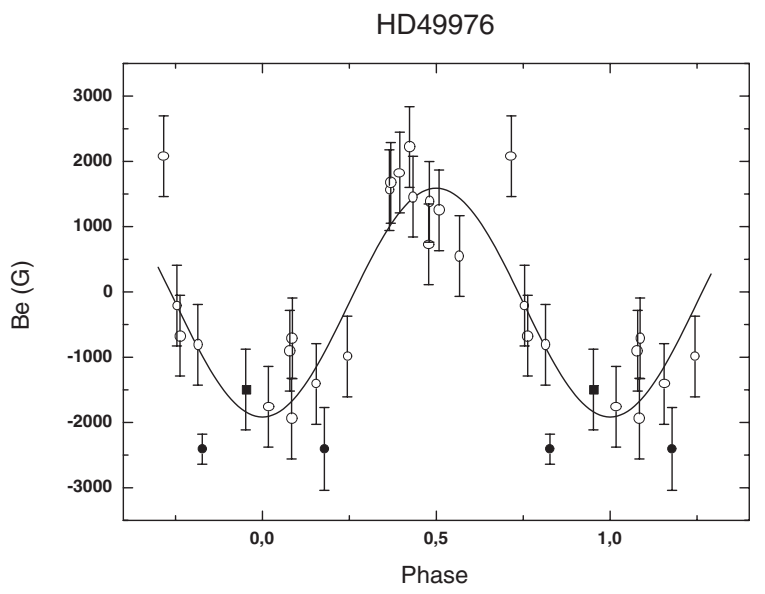

Fig. A.58. HD 49976. We used the period $P=2.18010^{\mathrm{d}}$, taken from Catalano \& Leone (1994). Magnetic variability of this star was investigated in Ref. 92. Open circles - Ref. 92, filled circles - Ref. 184, filled squares - Ref. 91.

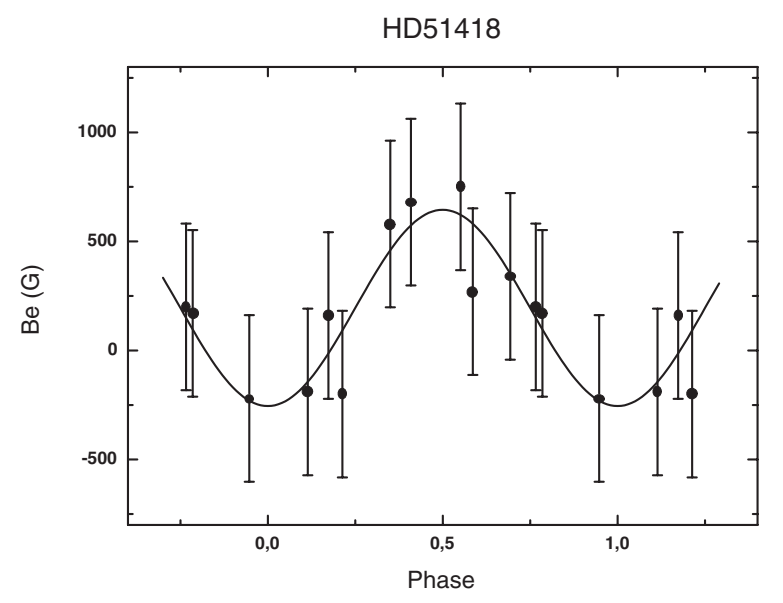

Fig. A.59. HD 51418. Behavior of $B_{\mathrm{e}}$ vs. phase $\phi$ was studied in Ref. 29 using the photograpic technique. Musielok \& Madej (1988) determined the period $P_{\mathrm{mag}}=5.4379^{\mathrm{d}}$, however, we adapted the magnetic period $P_{\text {mag }}=5.631^{\mathrm{d}}$. New measurements of high precision are necessary to improve the period and the phase curve.

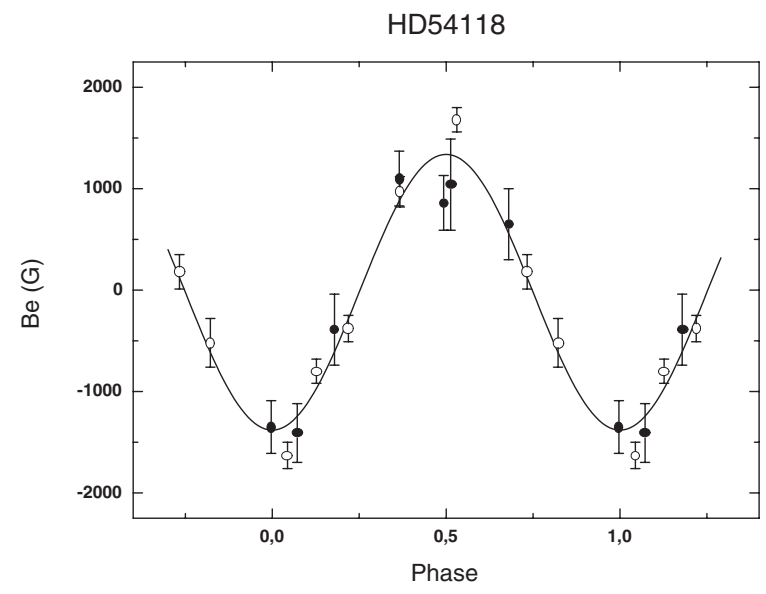

Fig. A.60. HD 54118. We used the magnetic period $P_{\mathrm{mag}}=5.631^{\mathrm{d}}$ taken from Bohlender et al. (1993). Open circles - Ref. $81\left(\mathrm{H}_{\beta}\right)$, filled circles - Ref. $230\left(\mathrm{H}_{\alpha}\right)$.

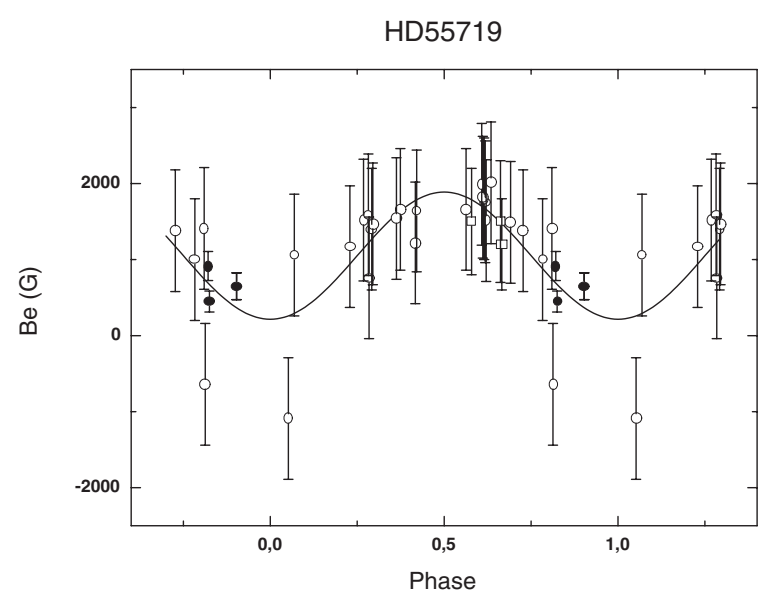

Fig. A.61. HD 55719. Bonsack (1976), Ref. 32, claims that the best magnetic rotational $P_{\mathrm{mag}}$ is in the range $30.39^{\mathrm{d}}-36.48^{\mathrm{d}}$. We determined and used here the other, more credible value $P_{\text {mag }}=1.00335^{\mathrm{d}}$. Open circles - Ref. 32, filled circles - Ref. 256, filled squares Ref. 93. 
V. D. Bychkov et al.: A catalog of stellar magnetic rotational phase curves, Online Material p 26

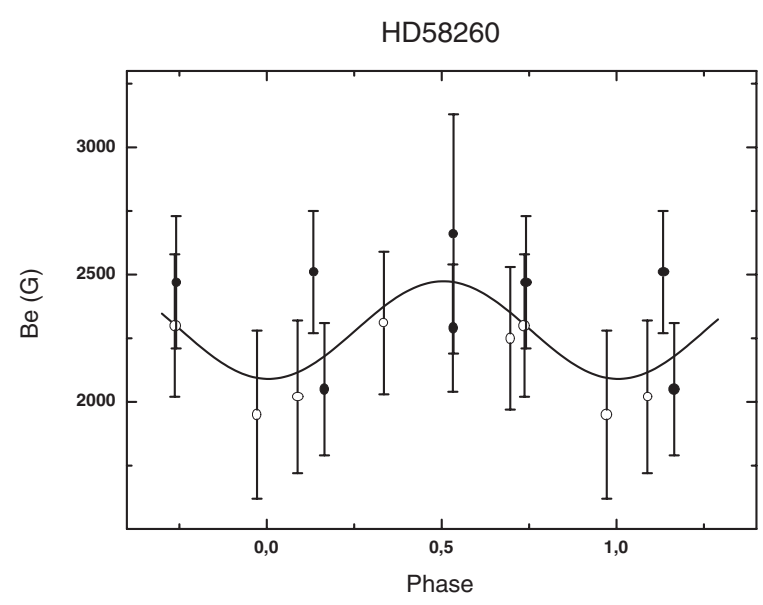

Fig. A.62. HD 58260. We used the period $P=1.657^{\mathrm{d}}$, taken from Pedersen (1979). Magnetic variability of this star was measured in hydrogen lines. The star has strong positive $B_{\mathrm{e}}$ field with moderate amplitude of variations. Open circles - Ref. 24, filled circles - Ref. 135.

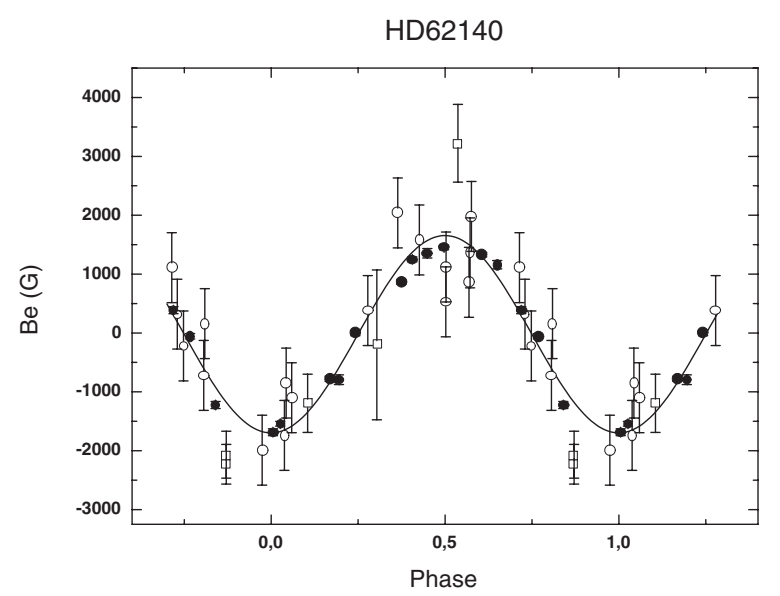

Fig. A.63. HD 62140. The best magnetic period $P_{\mathrm{mag}}=4.28488^{\mathrm{d}}$. It is close to the period $P_{\mathrm{mag}}=4.2867^{\mathrm{d}}$ obtained by Leroy (1995). The figure displays all the available $B_{\mathrm{e}}$ observations. Open circles - Ref. 62, filled squares - Ref. 91, filled circles - Ref. 310. Points of high accuracy exhibit systematic deviations from the phase curve around $\phi=0.5$.

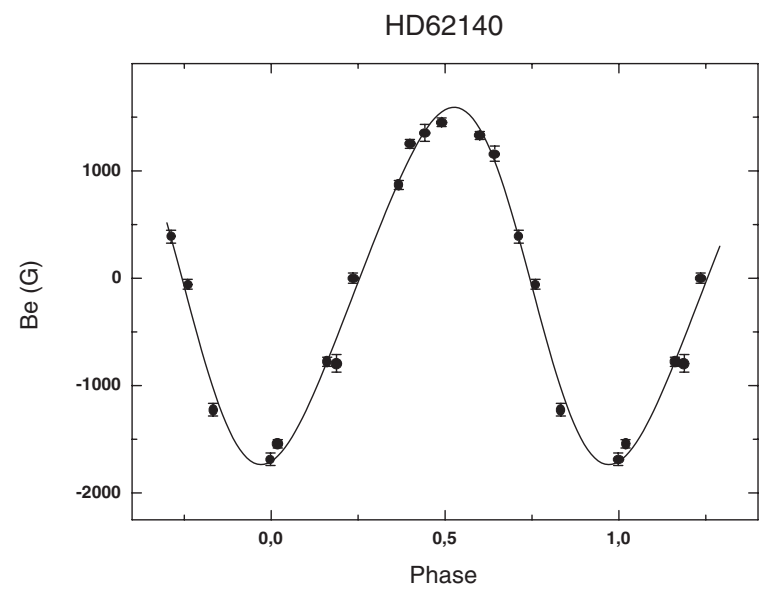

Fig. A.64. HD 62140. Magnetic period $P_{\text {mag }}=4.28488^{\mathrm{d}}$. Figure displays magnetic $B_{\mathrm{e}}$ points of high accuracy from Ref. 310 . The phase curve is described by double wave.

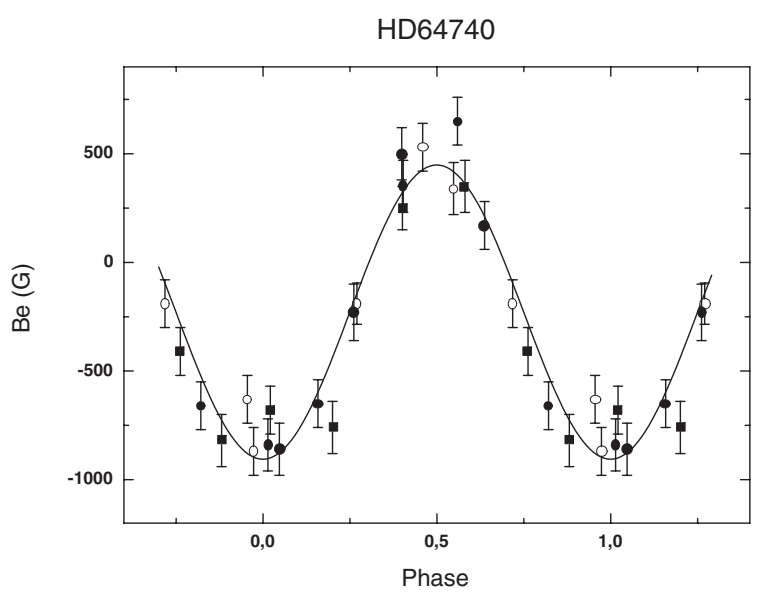

Fig. A.65. HD 64740. We used the period $P_{\mathrm{mag}}=1.657^{\mathrm{d}}$, taken from Shore \& Brown (1990). Open circles - Ref. 24 ( $\mathrm{H}_{\beta}$ line), filled circles - Ref. $135\left(\mathrm{H}_{\beta}\right)$, filled squares - Ref. 135 (He I 5876).

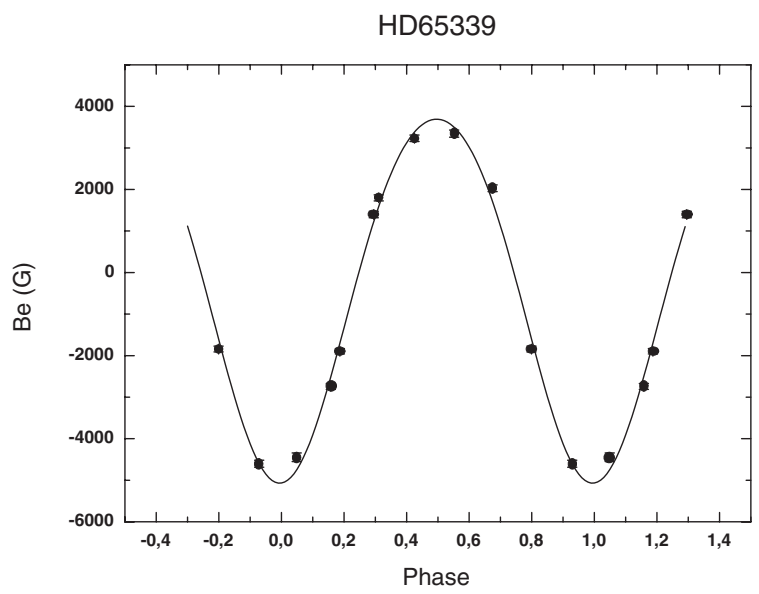

Fig. A.66. HD $65339=53 \mathrm{Cam}$. We used the period $P_{\mathrm{mag}}=8.0267^{\mathrm{d}}$, taken from Musielok \& Madej (1988). The figure presents the most accurate $B_{\mathrm{e}}$ measurements obtained in Ref. 310 from metal lines with the LSD method. We note systematic deviation of phase curve from a simple sine wave near $\phi \approx 0.5$. Therefore, we fitted $B_{\mathrm{e}}(\phi)$ phase curve by a double wave. 
V. D. Bychkov et al.: A catalog of stellar magnetic rotational phase curves, Online Material p 27

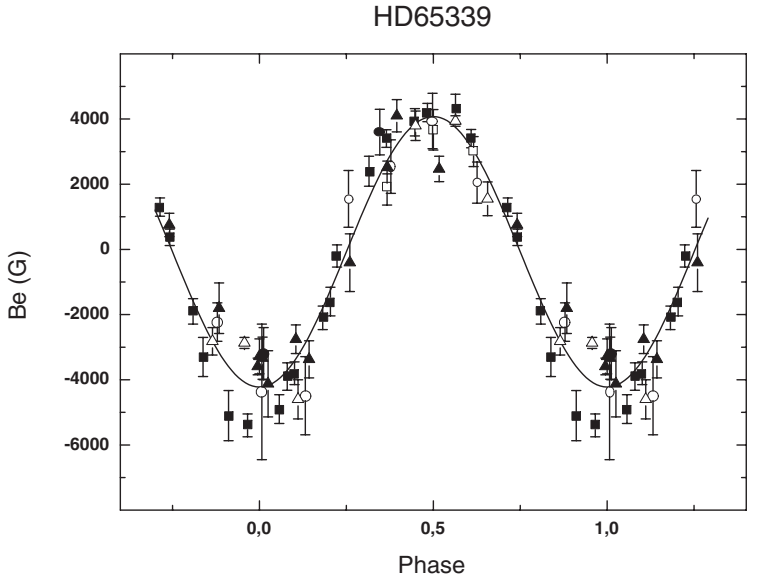

Fig. A.67. HD $65339=53 \mathrm{Cam}$. The period $P_{\mathrm{mag}}=8.0267^{\mathrm{d}}$ was used here. Phase curve $B_{\mathrm{e}}(\phi)$ was derived from hydrogen line measurements. We did not find any significant deviations from a sine wave. This is most likely due to lower accuracy of $B_{\mathrm{e}}$ points, than in the previous figure. Open circles - Ref. $24\left(\mathrm{H}_{\beta}\right)$, filled circles - Ref. $135\left(\mathrm{H}_{\beta}\right)$, filled squares - Ref. 135.

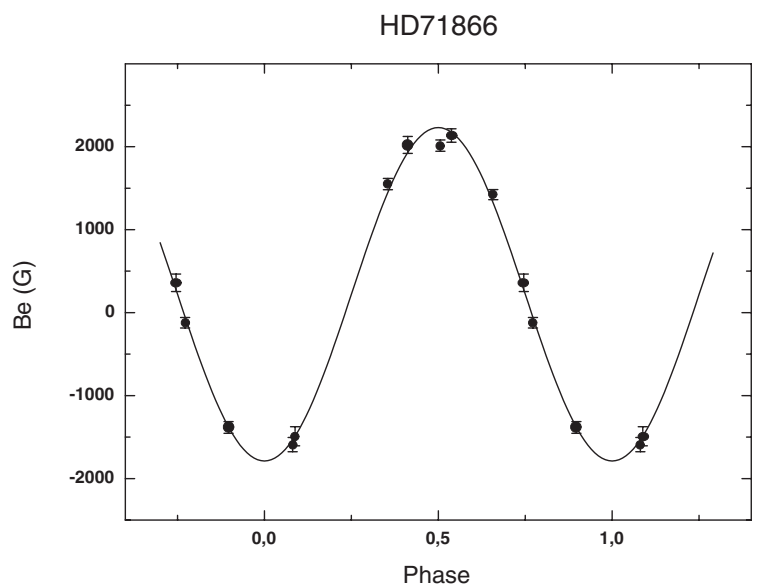

Fig. A.68. HD 71866. The best magnetic period $P_{\mathrm{mag}}=6.80024^{\mathrm{d}}$ was used here. It is close to the period $P_{\text {mag }}=6.80022^{\mathrm{d}}$, taken from Bagnulo et al. (1995). We plot here only the most accurate LSD points from Ref. 310.
HD72968

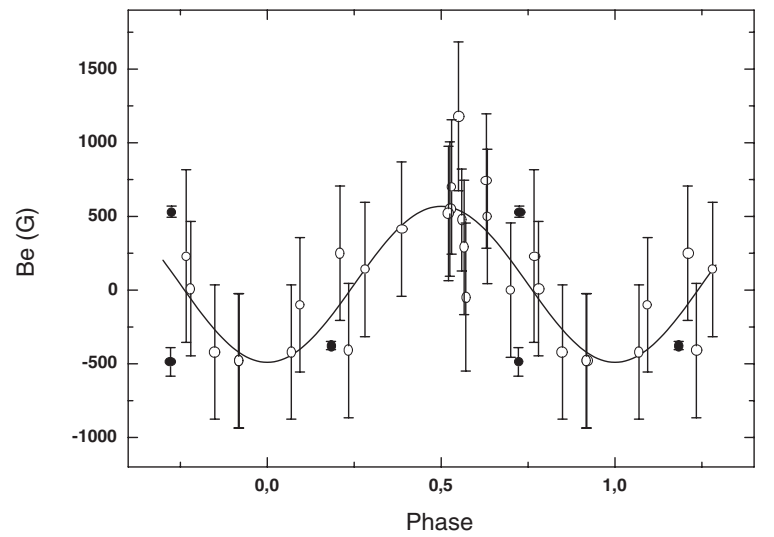

Fig. A.69. HD 72968. We used the best magnetic period $P_{\mathrm{mag}}=4.66458^{\mathrm{d}}$, which is close to the period $P_{\mathrm{mag}}=4.606^{\mathrm{d}}$ by Steinitz \& Pyper (1970). Magnetic field measurements which were obtained by photographic method - Refs. 1 and 91, are not accurate enough. More $B_{\mathrm{e}}$ points of high precision are necessary to define a more accurate magnetic phase curve. Open circles - Ref. 1, filled circles - Ref. 327, open squares - Ref. 91.

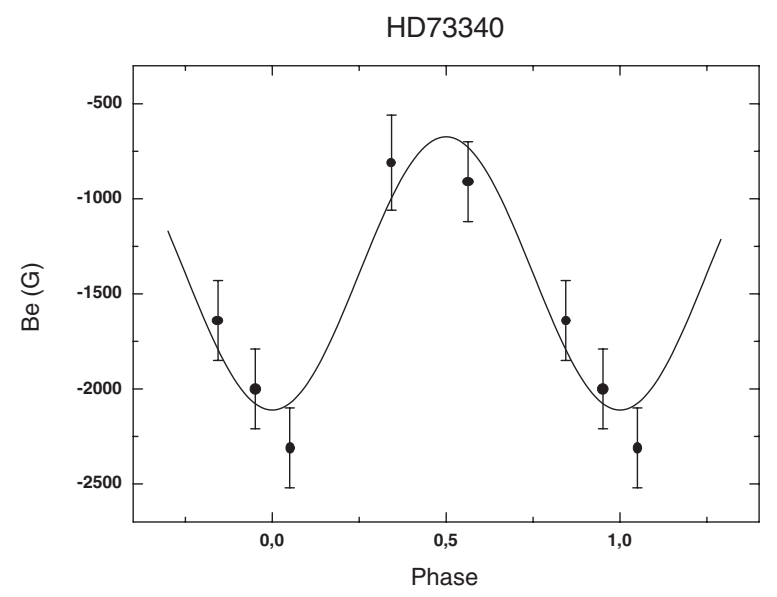

Fig. A.70. HD 73340. We used the period $P_{\text {mag }}=2.66753^{\mathrm{d}}$, taken from Bohlender et al. (1993). The number of $B_{\mathrm{e}}$ points is low, additional observations are necessary. 
V. D. Bychkov et al.: A catalog of stellar magnetic rotational phase curves, Online Material p 28

HD74521

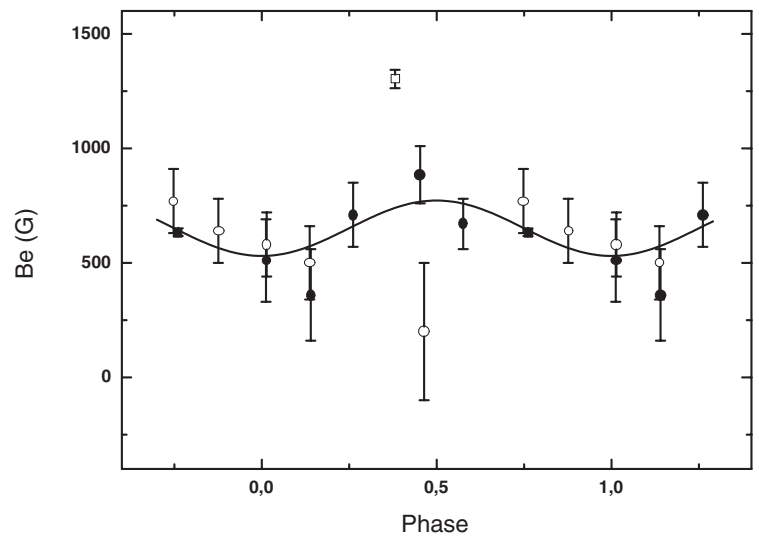

Fig. A.71. HD 74521. We used the photometric period $P_{\text {phot }}=7.76851^{\mathrm{d}}$, (Catalano \& Leone 1993). Magnetic field $B_{\mathrm{e}}$ and the amplitude of its variations are small, and the photographic $B_{\text {e }}$ points of low accuracy were not used here (Ref. 1). Open circles - Ref. 230, filled circles - Ref. 184, open squares - Ref. 327, filled squares - Ref. 333

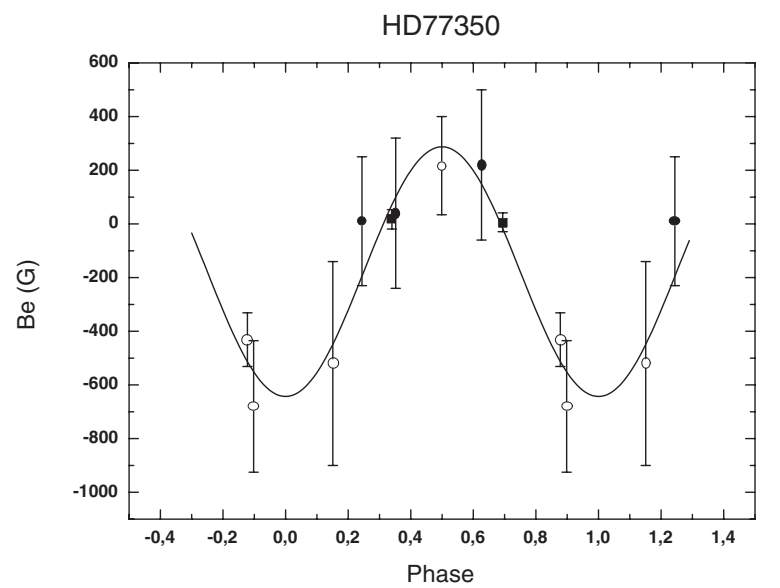

Fig. A.72. HD 77350. We used the period $P_{\text {mag }}=4.024^{\mathrm{d}}$, it is close to the photometric period $P_{\text {phot }}=4.191^{\mathrm{d}}$ (Kozlova et al. 1975). Magnetic field $B_{\mathrm{e}}$ and the amplitude of its variations are small, therefore, we did not use photographic $B_{\mathrm{e}}$ points of low accuracy (Ref. 1). Open circles - Ref. 327, filled circles - Ref. 230, filled squares - Ref. 333.

\section{HD78316}

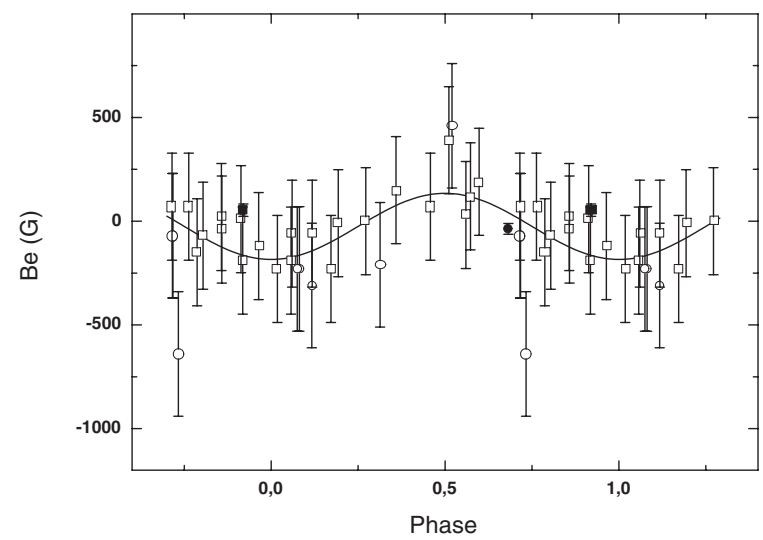

Fig. A.73. HD 78316. The period $P_{\mathrm{mag}}=5.0035^{\mathrm{d}}$ was adapted from Ref. 11. Open circles - Ref. 1, filled circles - Ref. 11, open squares Ref. 333, filled squares - Ref. 330.

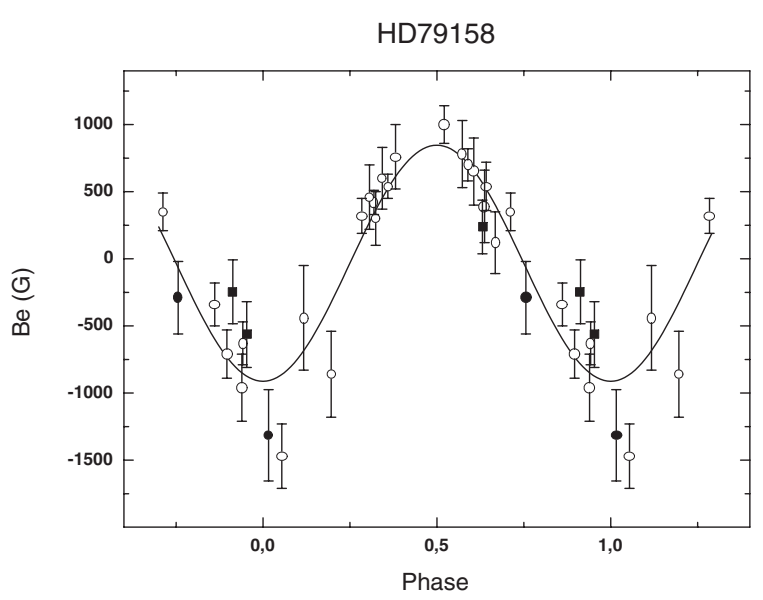

Fig. A.74. HD 79158. We used the best magnetic period $P_{\text {mag }}=3.8345^{\mathrm{d}}$, which is close to the photometric period $P_{\text {phot }}=3.83483^{\mathrm{d}}$, taken from Bohlender (1994). Magnetic field $B_{\mathrm{e}}$ measurements were obtained from $\mathrm{H}_{\beta}$ line. Open circles Ref. 181, filled circles - Ref. 37, filled squares - Ref. 327.

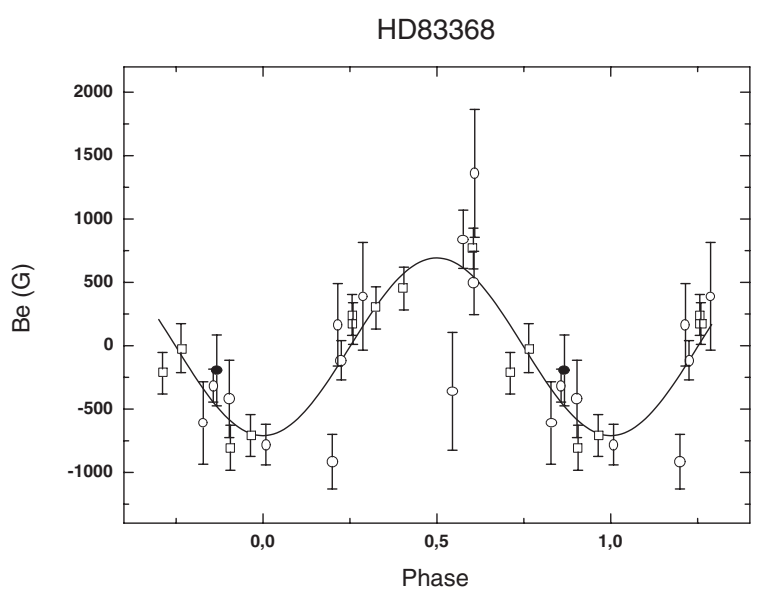

Fig. A.75. HD 83368. We used the period $P_{\mathrm{mag}}=2.851962^{\mathrm{d}}$, taken from Mathys (1991). Behavior of $B_{\mathrm{e}}$ in this star was investigated by various methods. Open circles - Ref. 184 (metal lines), filled circles Ref. 256 (metal lines), open squares - Ref. $186\left(\mathrm{H}_{\beta}\right.$ line). 
V. D. Bychkov et al.: A catalog of stellar magnetic rotational phase curves, Online Material p 29

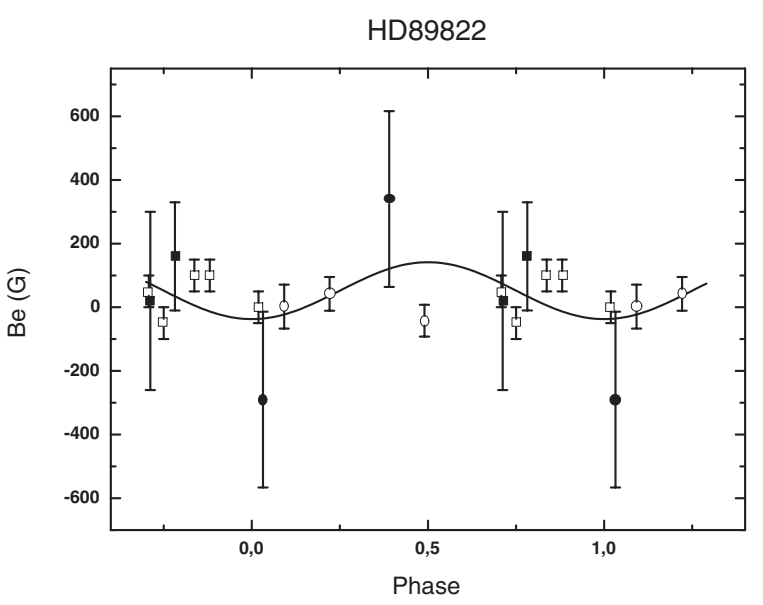

Fig. A.76. HD 89822. We used the period $P_{\text {mag }}=7.5586^{\mathrm{d}}$, taken from Catalano \& Leone (1991). The star is a spectroscopic binary. Magnetic properties were investigated with various methods. Measurements from Ref. 109 were not used due to low accuracy. Open circles Refs. 2, 230 (H lines), filled circles - Ref. 1 (metal lines, phot. method), open squares - Ref. 20 (metal line, only for primary star), filled squares - Refs. 237, 330 (metal lines, CCD images).

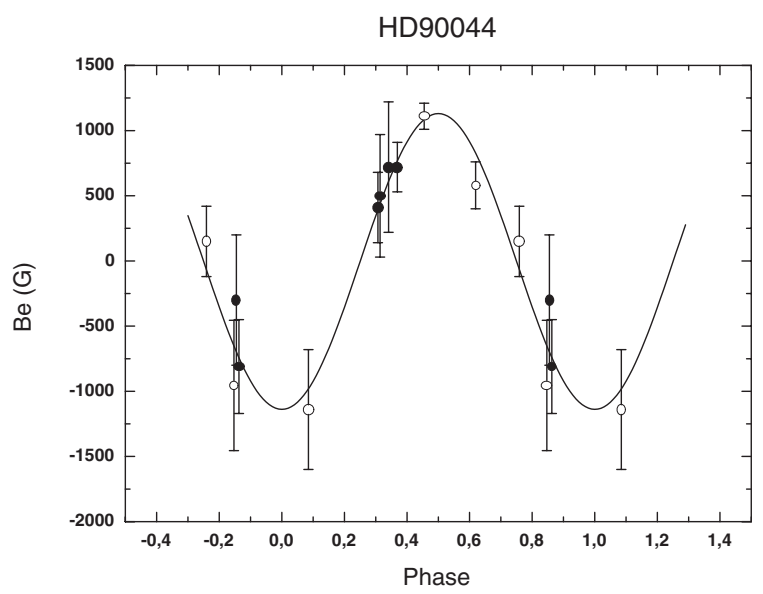

Fig. A.77. HD 90044. The period $P_{\mathrm{mag}}=4.379^{\mathrm{d}}$ was adapted from Ref. 324. Open circles - Ref. 324 (metal lines), filled circles - Ref. 230 (H lines).

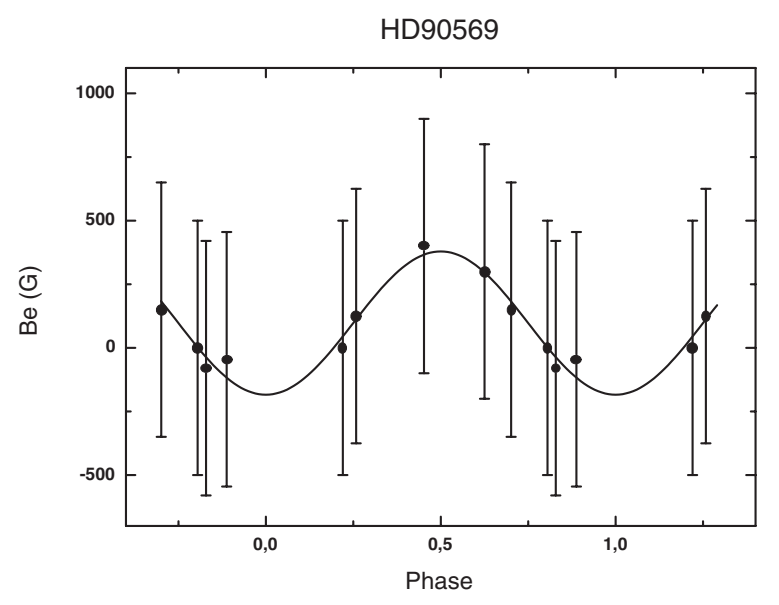

Fig. A.78. HD 90569. We used the period $P_{\mathrm{mag}}=1.445^{\mathrm{d}}$, taken from Wolff \& Morrison (1975). Determinations of $B_{\mathrm{e}}$ are scarce, existing data suggest that the longitudinal magnetic field does not vary significantly. New high accuracy magnetic field data are necessary to improve parameters of the magnetic variability.

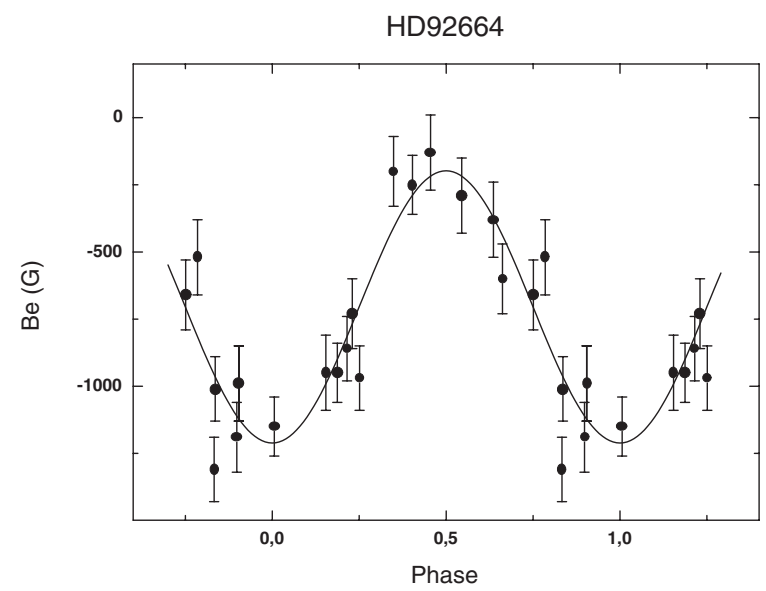

Fig. A.79. HD 92664. We used the period $P_{\mathrm{mag}}=1.67315^{\mathrm{d}}$, taken from Bohlender et al. (1993). Magnetic field $B_{\mathrm{e}}$ was measured in hydrogen lines - Refs. 81 and 230. The value of $B_{\mathrm{e}}$ from Ref. 81 was not used here due to low accuracy.

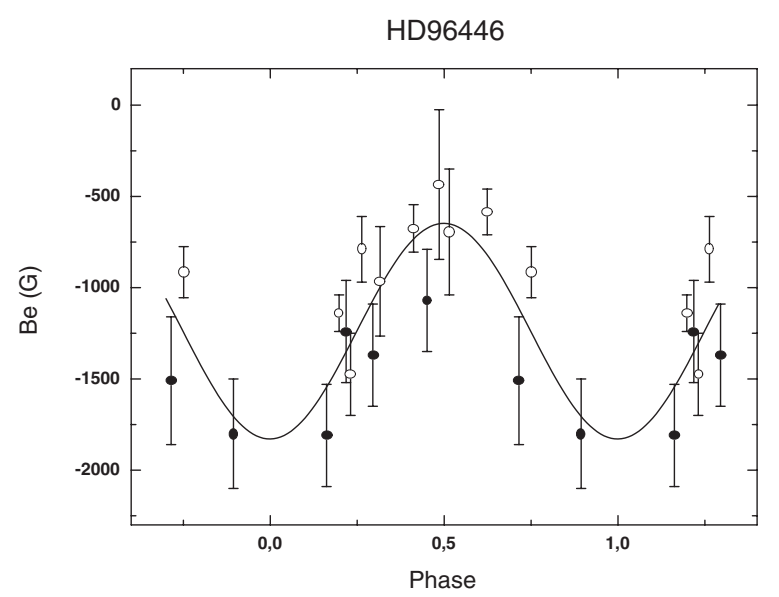

Fig. A.80. HD 96446. We determined and applied here the period $P_{\text {mag }}=0.85521^{\mathrm{d}}$. It is close to the period $P_{\mathrm{mag}}=0.85137^{\mathrm{d}}$, given by Mathews \& Bohlender (1991). Measurements of $B_{\mathrm{e}}$ in hydrogen lines yield systematically larger values that those in metal lines. Open circles - Ref. 184 (metal lines), filled circles - Ref. 24 (H lines). 
V. D. Bychkov et al.: A catalog of stellar magnetic rotational phase curves, Online Material p 30

HD96707

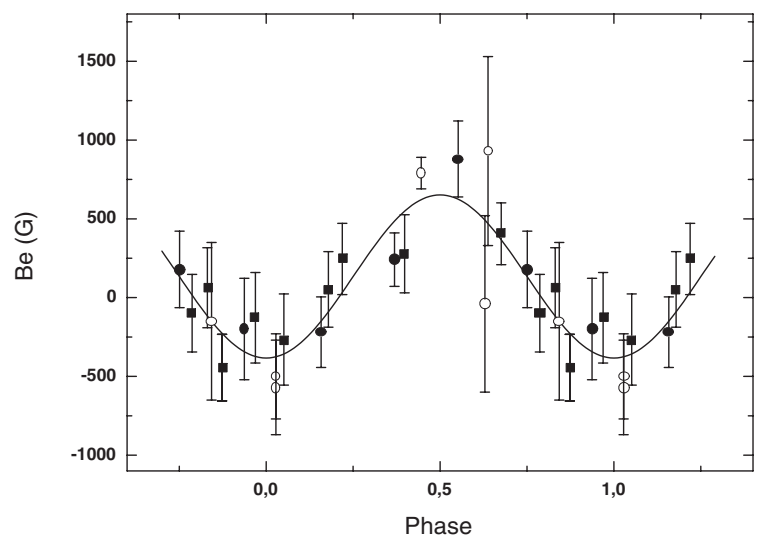

Fig. A.81. HD 96707. The present magnetic period $P_{\mathrm{mag}}=2.49183^{\mathrm{d}}$. It is close to the previous period $P_{\mathrm{mag}}=2.55305^{\mathrm{d}}$, taken from Bychkov et al. (1997a), Ref. 260. Additional high accuracy magnetic observations are necessary to improve phase curve $B_{\mathrm{e}}(\phi)$. Averaged curve was plotted without $B_{\mathrm{e}}$ points from Ref. 91, which are of low accuracy. Open circles - Ref. 324 (metal lines), filled circles - Ref. 260 (metal lines), filled squares - Ref. 260 (H lines).

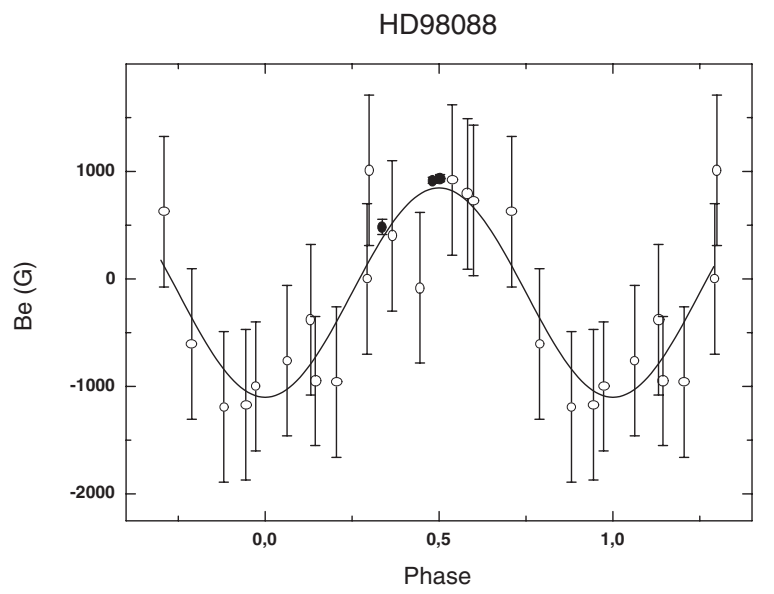

Fig. A.82. HD 98088. We determined and used here the magnetic period $P_{\mathrm{mag}}=5.81714^{\mathrm{d}}$. It is nearly equal to the period $P=5.905130^{\mathrm{d}}$, taken from Catalano \& Leone (1994). Additional high precision $B_{\mathrm{e}}$ observations are necessary to improve parameters of the magnetic variability. Open circles - Refs. 1, 108 (metal lines, phot. method), filled circles - Ref. 310 (metal lines, LSD method).

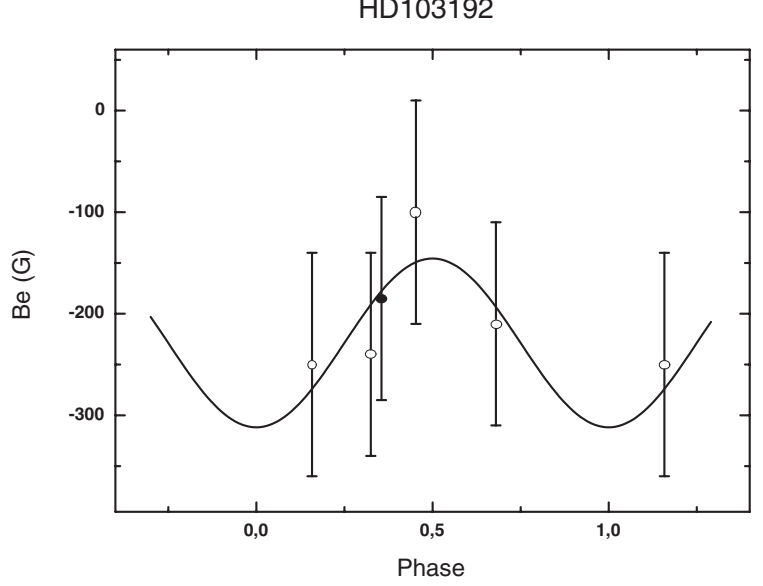

Fig. A.83. HD 103192. We used the period $P_{\text {mag }}=2.344^{\mathrm{d}}$, taken from Manfroid \& Renson (1980). More $B_{\mathrm{e}}$ observations are necessary to improve parameters of the magnetic variability. Open circles Ref. 230 (H lines), filled circles - Ref. 2 (H lines).

\section{HD108662}

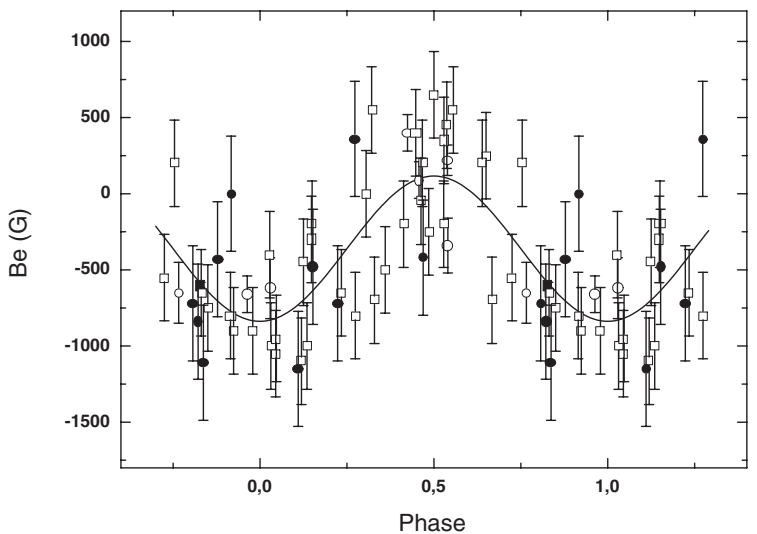

Fig. A.84. HD 108662. We used the period $P_{\mathrm{mag}}=5.0805^{\mathrm{d}}$, taken from Rice \& Wehlau (1994). Magnetic field $B_{\mathrm{e}}$ was observed essentially with photographic method, there exists only the single measurement made with the LSD method. More $B_{\mathrm{e}}$ measurements of high precision are necessary to obtain more accurate parameters of magnetic variability. Open circles - Ref. 118, filled circles - Ref. 1, open squares - Ref. 11, filled squares - Ref. 333. 
V. D. Bychkov et al.: A catalog of stellar magnetic rotational phase curves, Online Material p 31

HD108945

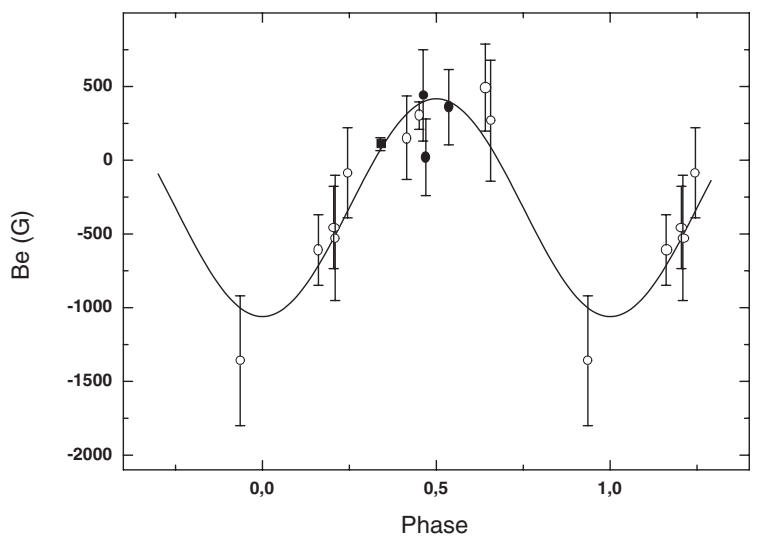

Fig. A.85. HD 108945. We determined and used here the most probable magnetic period $P_{\mathrm{mag}}=1.92442^{\mathrm{d}}$. It is close to the period $P_{\text {mag }}=1.83736^{\mathrm{d}}$, taken from Zverko (1987). New high accuracy magnetic field data are necessary to determine parameters of $B_{\mathrm{e}}(\phi)$. Open circles - Ref. 327 (H lines), filled circles - Ref. 2 (H lines), filled squares - Ref. 333 (metal lines, LSD method).

\section{HD110379}

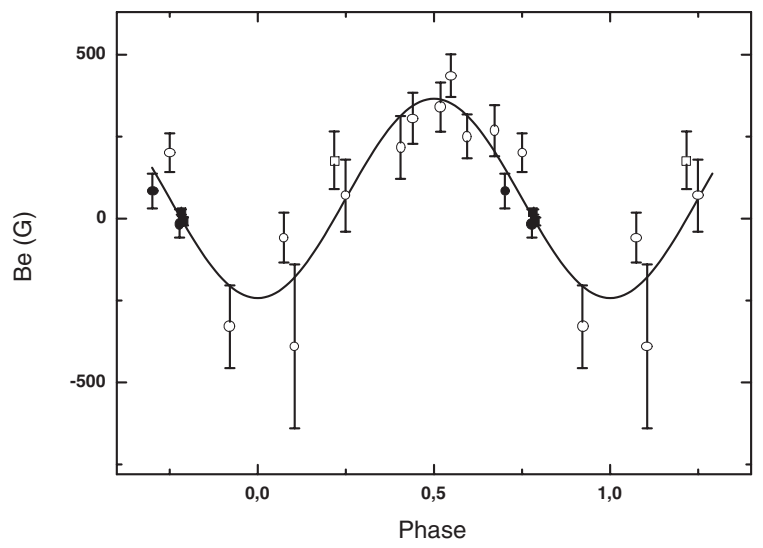

Fig. A.86. HD 110379. We determined the most probable period of magnetic variability $P_{\mathrm{mag}}=12.92696^{\mathrm{d}}$. Additional high precision $B_{\mathrm{e}}$ observations are necessary to determine precisely $B_{\mathrm{e}}(\phi)$. Open circles - Refs. 1, 61 (metal lines, phot. method), filled circles - Ref. 77 (H lines), filled squares - Ref. 333 (metal lines, LSD method), open squares - Ref. 327 (metal lines).

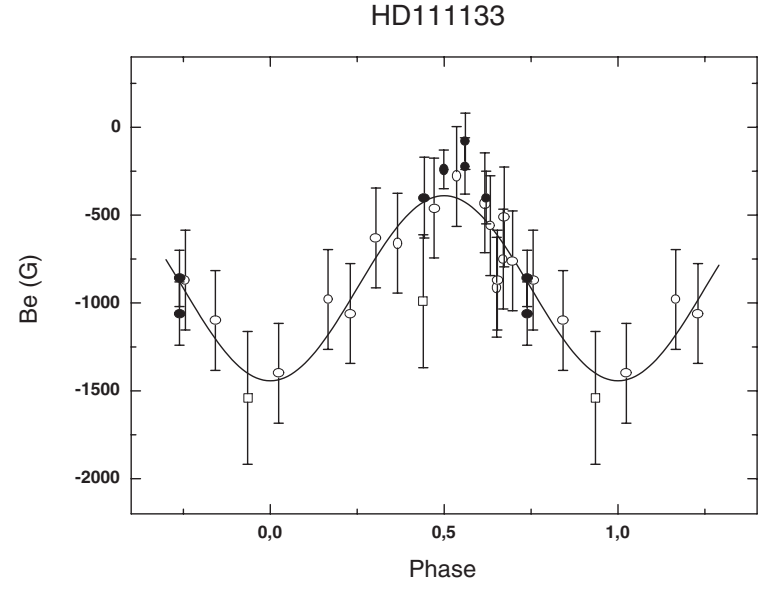

Fig. A.87. HD 111133. We used the period $P_{\mathrm{mag}}=16.3078^{\mathrm{d}}$, taken from North \& Adelman (1995). Magnetic behavior of this star was investigated with photographic methods only. Additional $B_{\mathrm{e}}$ measurements of high precision are necessary to improve parameters of magnetic variability. Open circles - Ref. 23, filled circles - Ref. 142, open squares - Ref. 1 .

\section{HD112185}

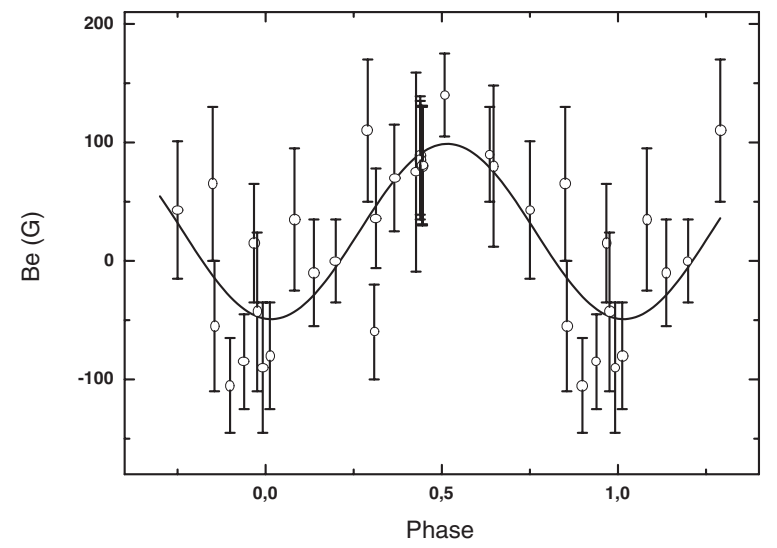

Fig. A.88. HD 112185. We used the period $P_{\mathrm{mag}}=5.0887^{\mathrm{d}}$, taken from Bohlender \& Landstreet (1990). Figure displays the $B_{\mathrm{e}}(\phi)$ phase curve based on measurements in hydrogen lines, which are of acceptable precision; cf. Refs. 2, 182, 271 and 334. 
V. D. Bychkov et al.: A catalog of stellar magnetic rotational phase curves, Online Material p 32

HD112185

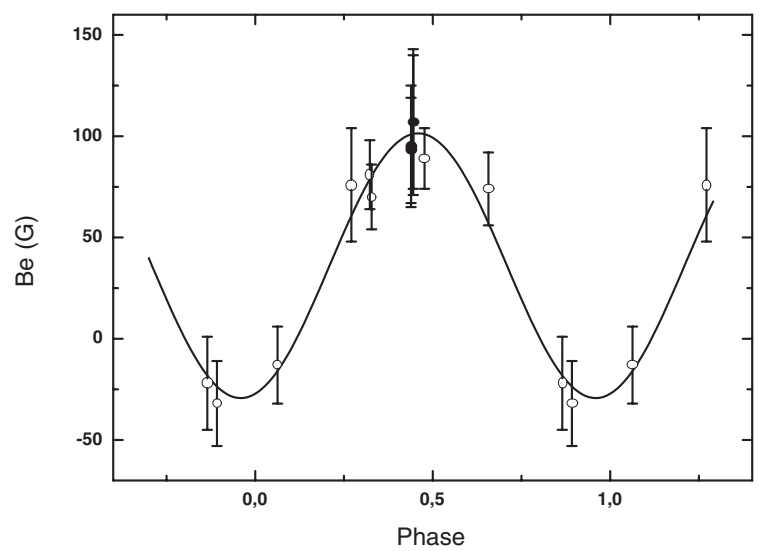

Fig. A.89. HD 112185. We used the period $P_{\text {mag }}=5.0887^{\mathrm{d}}$, taken from Bohlender \& Landstreet (1990). Figure displays the phase curve based on precise $B_{\mathrm{e}}$ points measured in metal lines. Open circles Ref. 310 (LSD method), filled circles - Ref. 334.

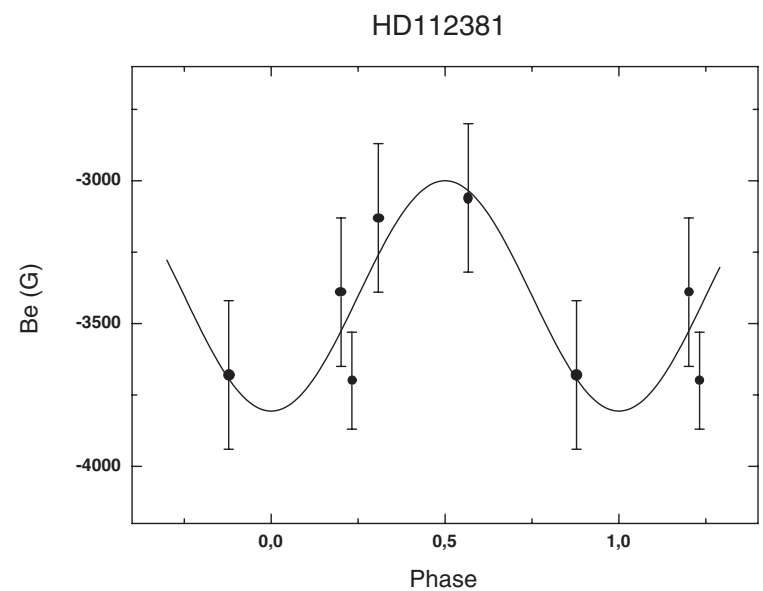

Fig. A.90. HD 112381. We used the period $P_{\mathrm{mag}}=2.84^{\mathrm{d}}$, taken from Bohlender et al. (1993). More $B_{\mathrm{e}}$ observations of high accuracy are necessary to obtain more accurate parameters of the phase curve. Filled circles - Ref. 230 (H lines).

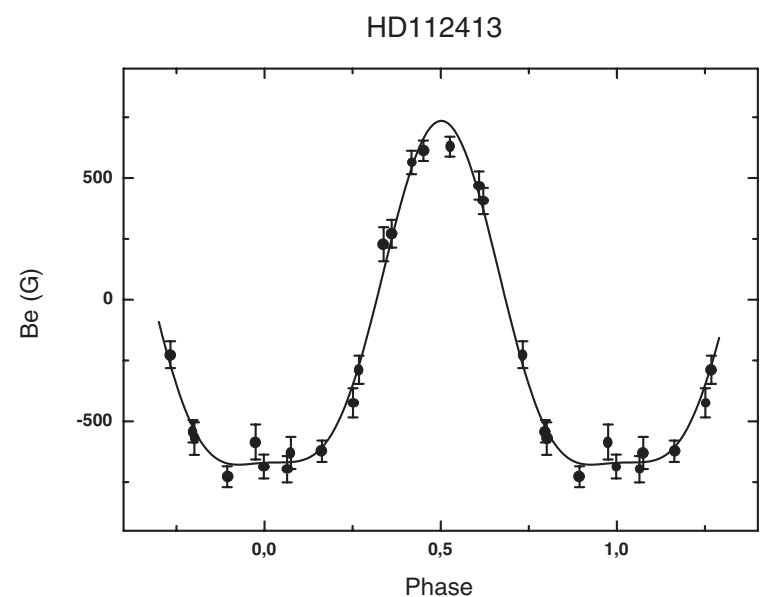

Fig. A.91. HD 112413. We used the period $P_{\mathrm{mag}}=5.46939^{\mathrm{d}}$, taken from Borra \& Vaughan (1978). The figure presents the rotational phase curve $B_{\mathrm{e}}(\phi)$ derived from $B_{\mathrm{e}}$ measurements of Ref. 310 (metal lines, LSD method). The averaged phase curve $B_{\mathrm{e}}(\phi)$ is a double wave.

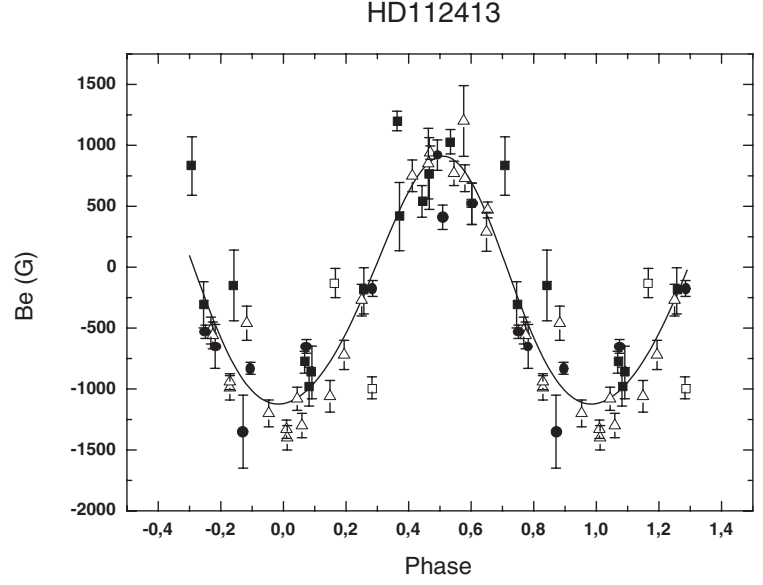

Fig. A.92. HD 112413. We used the period $P_{\mathrm{mag}}=5.46939^{\mathrm{d}}$, taken from Borra \& Vaughan (1978). Magnetic rotational phase curve was derived from $B_{\mathrm{e}}$ points measured in hydrogen lines, Refs. 25, 33, 77, 126, 215 and 327. The phase curve was fitted by a double wave. Open circles - Ref. 215, filled circles - Ref. 25, open squares - Ref. 126, filled squares - Ref. 327, open triangles - Ref. 33, filled triangles Ref. 77.

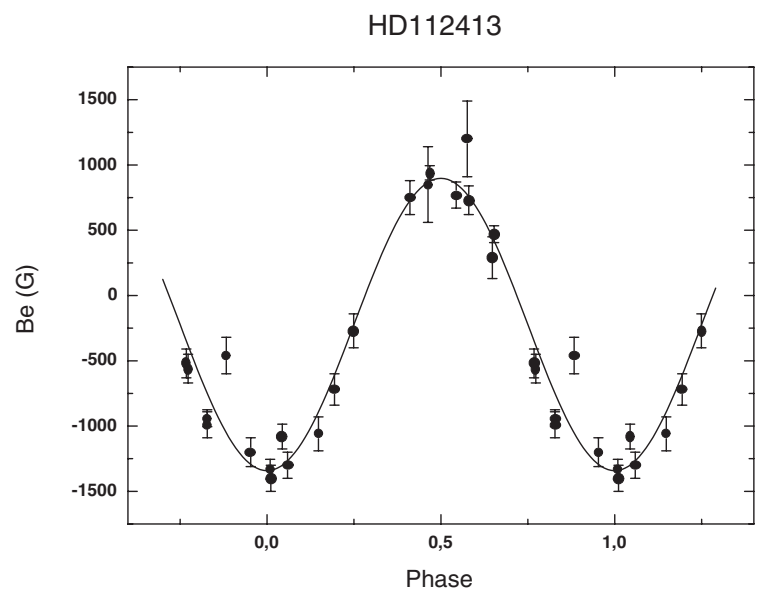

Fig. A.93. HD 112413. We used the period $P_{\mathrm{mag}}=5.46939^{\mathrm{d}}$ (Borra $\&$ Vaughan 1978). The figure shows that there exists a large scatter of the observed $B_{\mathrm{e}}$ measurements, which strongly increases uncertainty of the average phase curve. Magnetic $B_{\mathrm{e}}$ measurements were taken from Ref. 33, the phase curve takes form of a sine wave. 
V. D. Bychkov et al.: A catalog of stellar magnetic rotational phase curves, Online Material p 33

HD116458

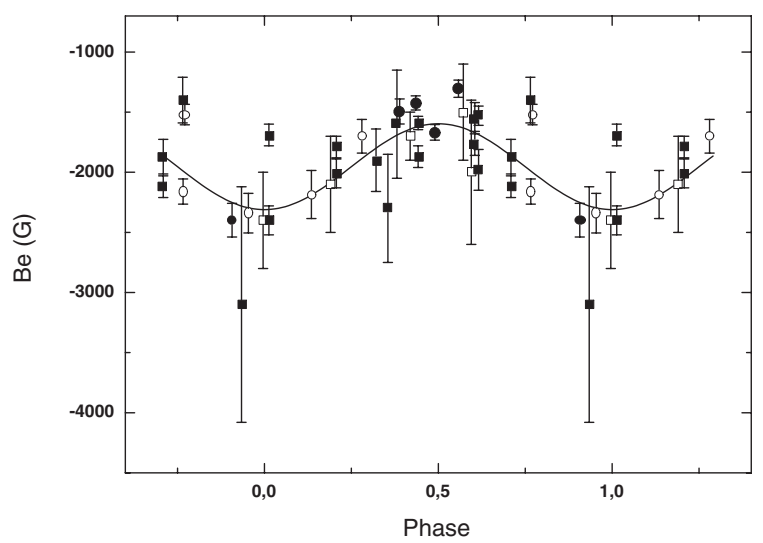

Fig. A.94. HD 116458. We determined the most probable period, $P_{\text {mag }}=2.3596^{\mathrm{d}}$. New magnetic observations are necessary to obtain the better period and phase curve. Open circles - Ref. 184, filled circles - Ref. 256, filled squares - Ref. 187, open squares - Ref. 93.

\section{HD118022}

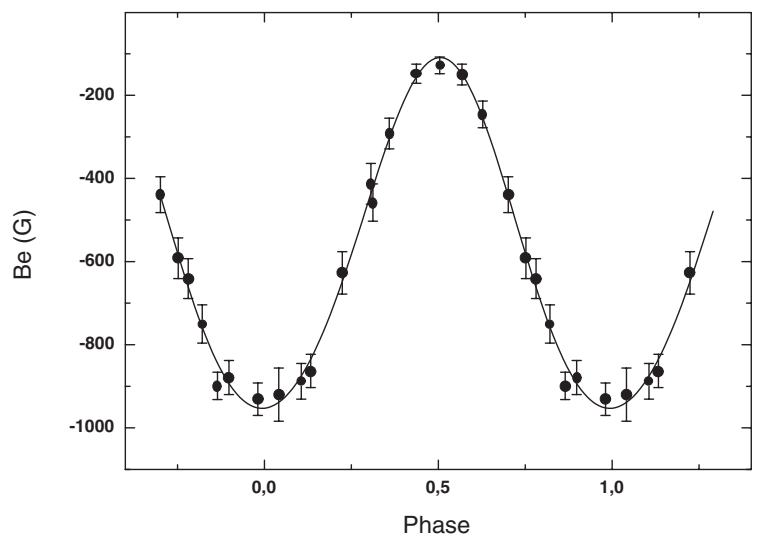

Fig. A.95. HD 118022. We used the period $P_{\text {mag }}=3.722084^{\mathrm{d}}$, taken from Catalano \& Leone (1994). Figure presents the $B_{\mathrm{e}}(\phi)$ curve obtained with LSD method, metal lines - Ref. 310. Averaged phase curve is fitted a by double wave.

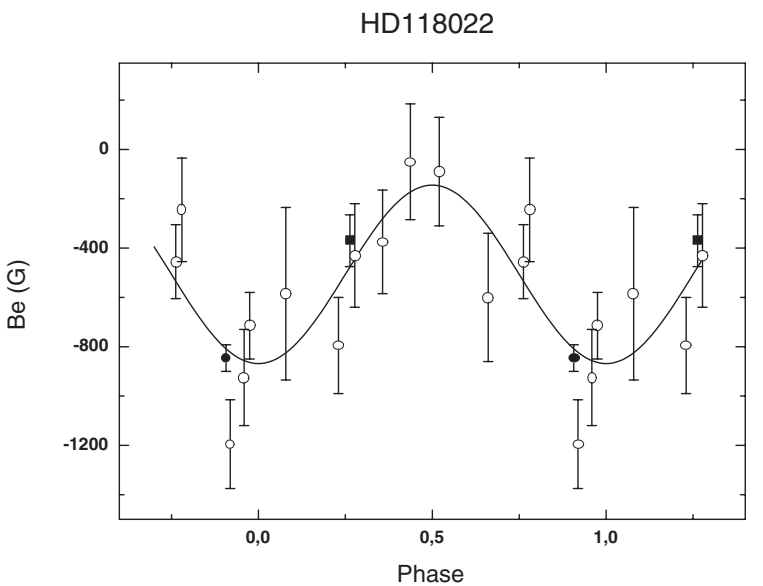

Fig. A.96. HD 118022. We used the period $P_{\mathrm{mag}}=3.722084^{\mathrm{d}}$ (Catalano \& Leone 1994). Phase curve was obtained from $B_{\mathrm{e}}$ points observed in hydrogen lines, and was approximated by a sine wave. Open circles - Ref. 2, filled circles - Ref. 77, filled squares - Ref. 76.

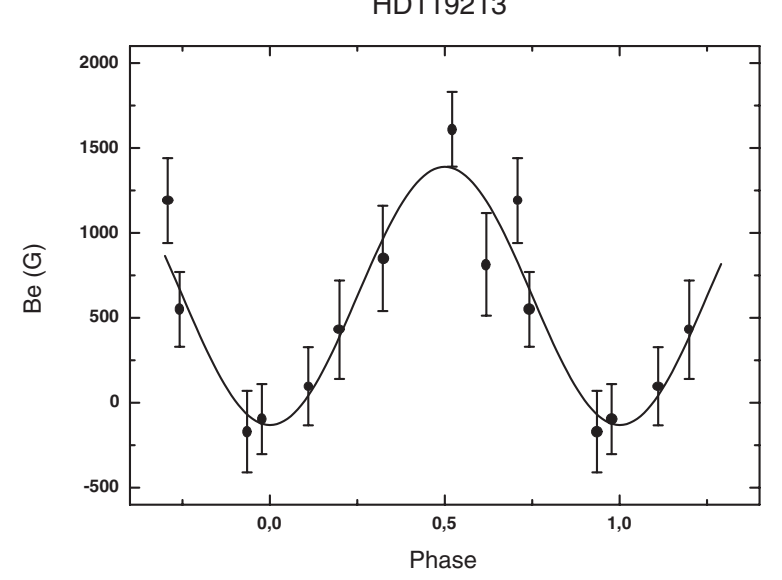

Fig. A.97. HD 119213. We used the period $P_{\mathrm{mag}}=2.44997^{\mathrm{d}}$, taken from Pyper \& Adelman (1985). Photographic magnetic field measurements which were obtained in metal lines, Ref. 113, did not yield any credible results. Magnetic behavior of this star could be understood from hydrogen line $B_{\mathrm{e}}$ measurements - Ref. 147.

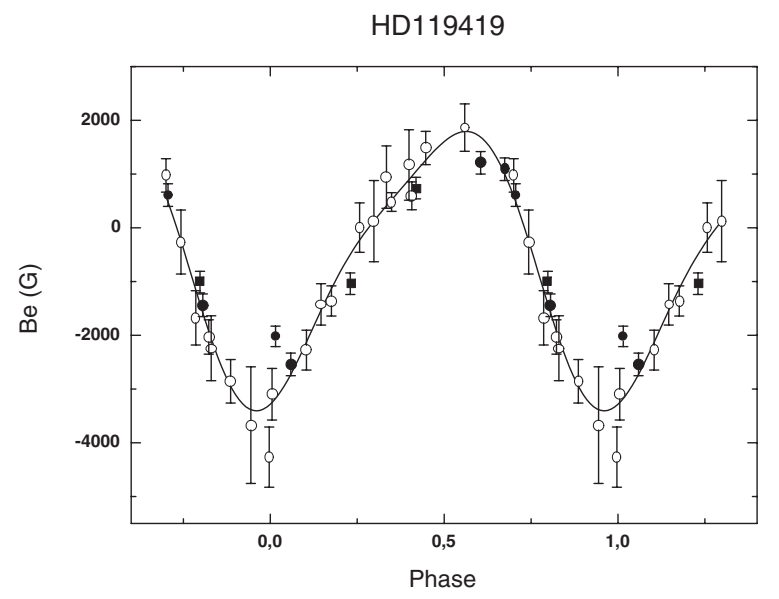

Fig. A.98. HD 119419. We used the period $P_{\mathrm{mag}}=2.6006^{\mathrm{d}}$, taken from Bohlender et al. (1993). Magnetic rotational phase curve is described by a double wave. There is no significant difference between $B_{\mathrm{e}}$ measurements observed both in metal lines and in $\mathrm{H}$ lines. Open circles - Ref. 184 (metal lines), filled circles - Ref. $230(\mathrm{H}$ lines), filled squares - Ref. 168 (H lines). 
V. D. Bychkov et al.: A catalog of stellar magnetic rotational phase curves, Online Material p 34

HD122532

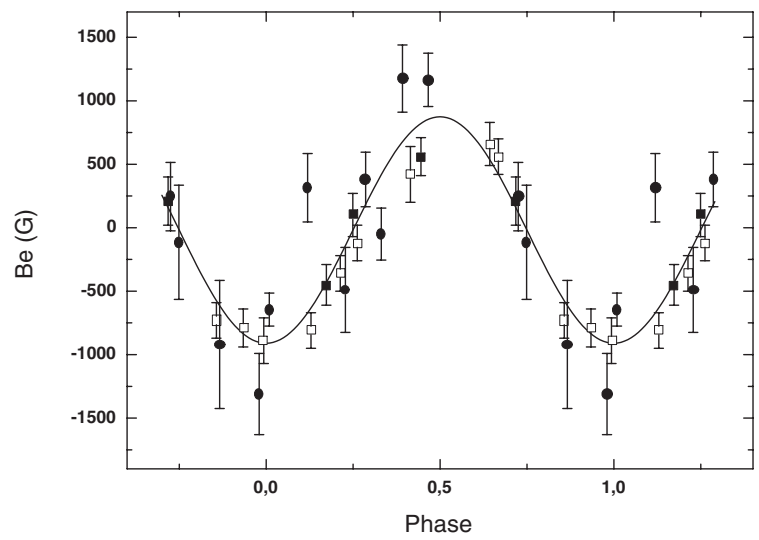

Fig. A.99. HD 122532. We determined and used here the best magnetic period $P_{\mathrm{mag}}=3.68137^{\mathrm{d}}$. It is close to the period $P_{\mathrm{mag}}=$ $3.6807^{\mathrm{d}}$, taken from Bohlender et al. (1993). Magnetic rotational phase curve $B_{\mathrm{e}}(\phi)$ is approximated by a sine wave. There are no noticeable systematic differences between $B_{\mathrm{e}}$ estimates obtained either in hydrogen or in metal lines. Hydrogen $B_{\mathrm{e}}$ observations exhibit larger scatter than $\mathrm{H}$ lines data. Filled circles - Ref. 184 (metal lines), open squares - Ref. 168 (H lines), filled squares - Ref. 230 (H lines).

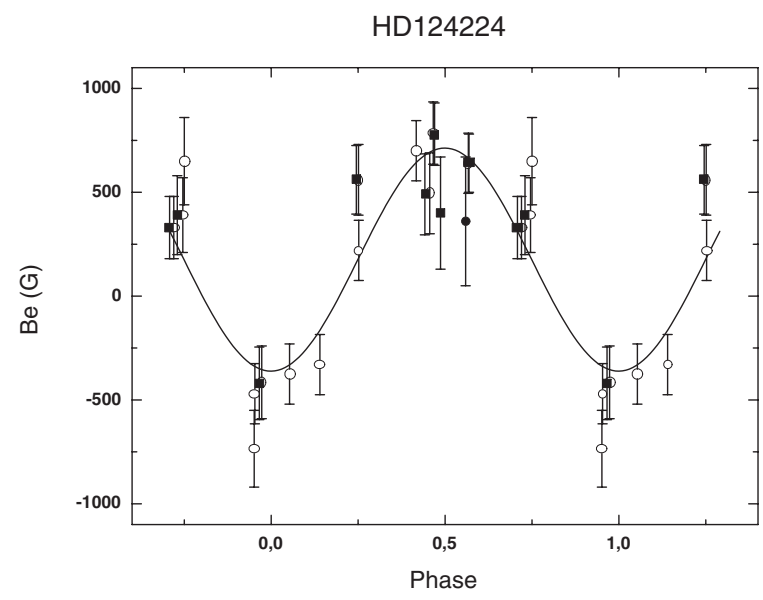

Fig. A.100. HD 124224. We used the period $P_{\mathrm{mag}}=0.520675^{\mathrm{d}}$ taken from Borra \& Landstreet (1980), Ref. 2. Magnetic field $B_{\mathrm{e}}$ was measured only in hydrogen lines: $\mathrm{H}_{\beta}-$ Refs. 2, 60; $\mathrm{H}_{\alpha}$ and $H_{\gamma}$ - Ref. 25. However, $\mathrm{H}_{\alpha}$ data from Ref. 25 were not used due to low accuracy. Open circles - Ref. 2, filled circles - Ref. 25, filled squares - Ref. 60.

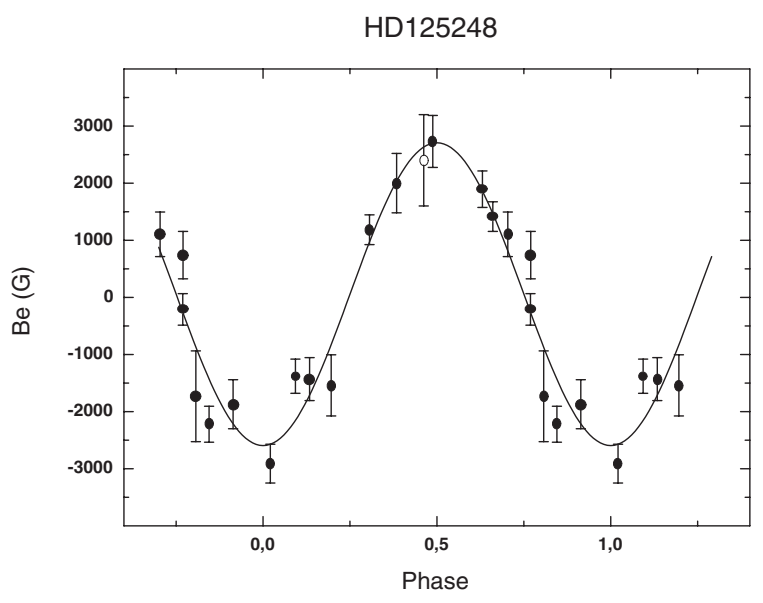

Fig. A.101. HD 125248. We used the period $P_{\mathrm{mag}}=9.2954^{\mathrm{d}}$, taken from Mathys (1994). Averaged phase curve was obtained from $B_{\mathrm{e}}$ points measured in hydrogen line wings. Open circles - Ref. 25, filled circles - Ref. 2.

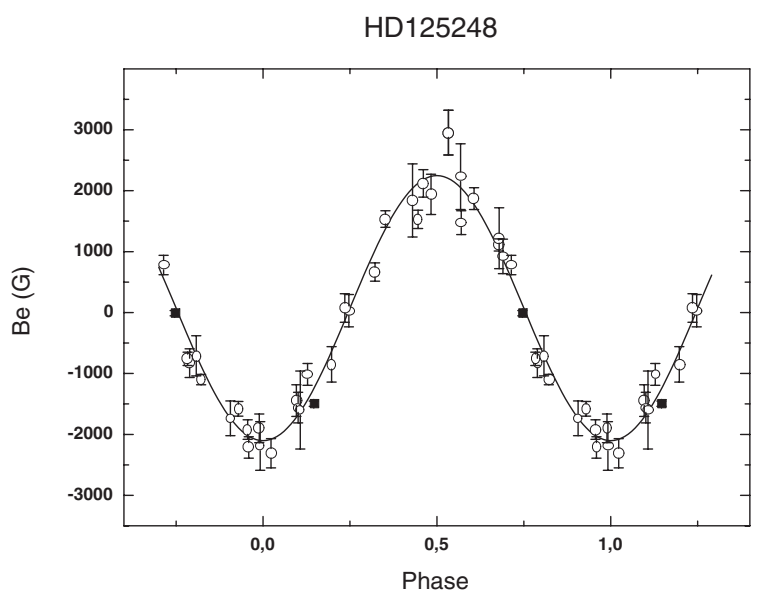

Fig. A.102. HD 125248. We used the period $P_{\mathrm{mag}}=9.2954^{\mathrm{d}}$, taken from Mathys (1994). Averaged phase curve $B_{\mathrm{e}}(\phi)$ was obtained from metal lines. Open circles - Refs. 184, 184, 239, 256 and 324. Filled circles - Ref. 333.

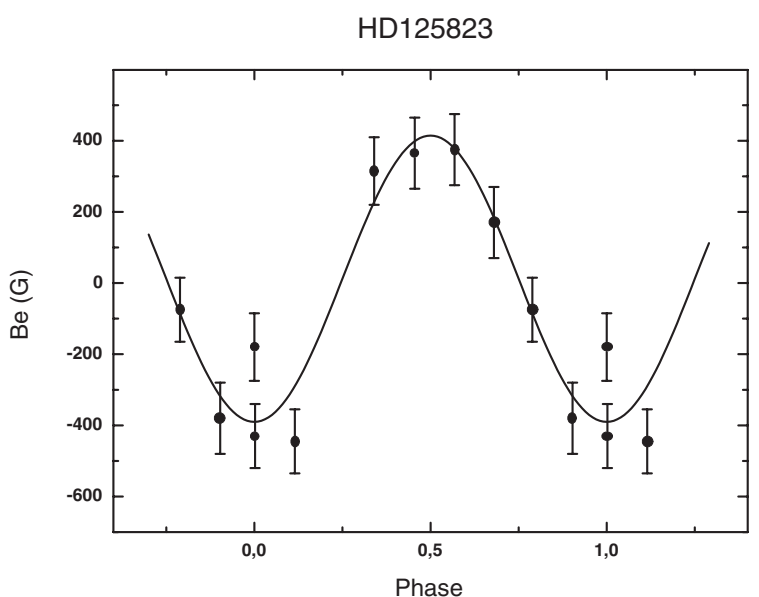

Fig. A.103. HD 125823. We used the period $P_{\mathrm{mag}}=8.8171^{\mathrm{d}}$, taken from Pedersen \& Thomsen (1977). Magnetic $B_{\mathrm{e}}(\phi)$ phase curve was obtained from hydrogen line $B_{\mathrm{e}}$ measurements, Ref. 37 . 
V. D. Bychkov et al.: A catalog of stellar magnetic rotational phase curves, Online Material p 35

HD125823

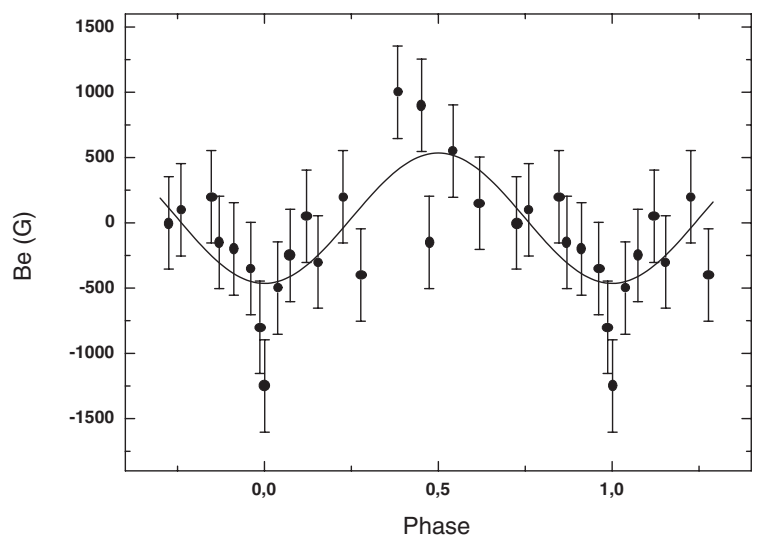

Fig. A.104. HD 125823. We used the period $P_{\mathrm{mag}}=8.8171^{\mathrm{d}}$, taken from Pedersen \& Thomsen (1977). Phase curve $B_{\mathrm{e}}(\phi)$ was obtained from metal line observations, Ref. 176.

HD126515

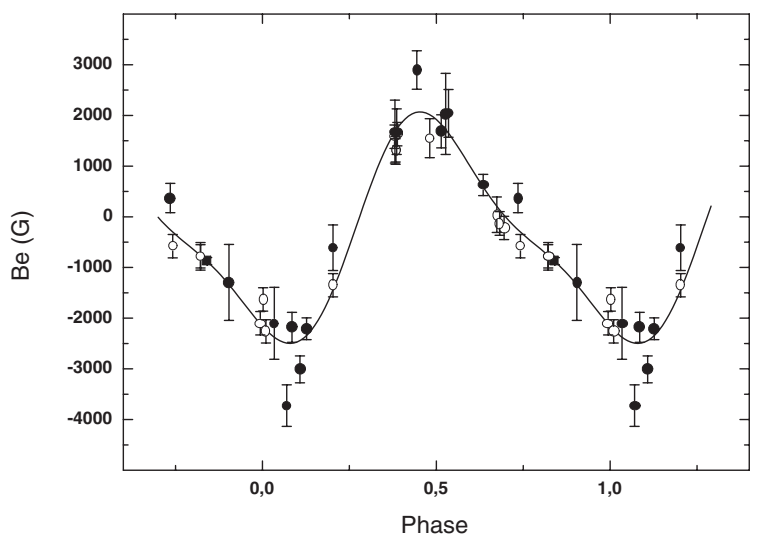

Fig. A.105. HD 126515. We used the period $P_{\mathrm{mag}}=129.95^{\mathrm{d}}$, taken from Mathys et al. (1986). Magnetic phase curve $B_{\mathrm{e}}(\phi)$ of this star is best fit by a double wave. Open circles - Refs. 1, 18 and 91 (metal lines, phot. method), filled circles - Refs. 184, 256 and 324 (metal lines), filled squares - Ref. 310 (metal lines, LSD method).

HD128898

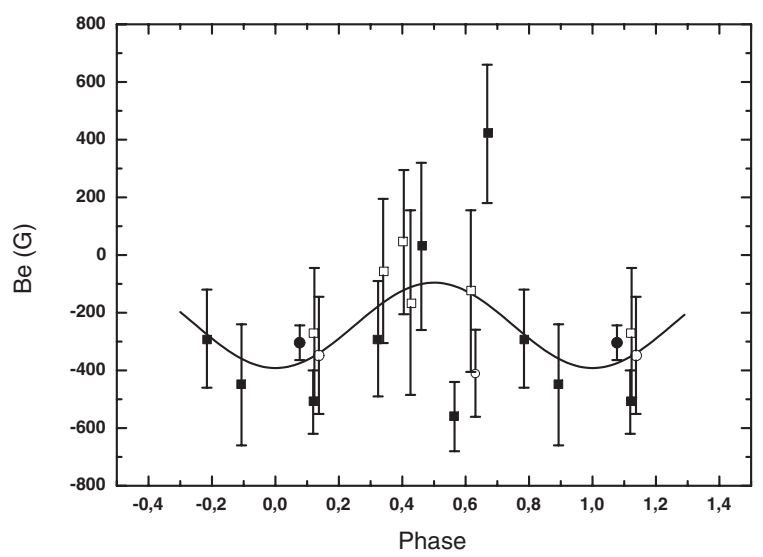

Fig. A.106. HD 128898. We determined and used the best magnetic period $P_{\mathrm{mag}}=4.4794^{\mathrm{d}}$. It is very close to the period $P_{\mathrm{mag}}=4.4790^{\mathrm{d}}$ by Kurtz et al. (1994). More $B_{\mathrm{e}}$ points of high accuracy are necessary to improve parameters of the phase curve. Open circles - Ref. 256, filled circles - Ref. 2, open squares - Ref. 184, filled squares - Ref. 81.

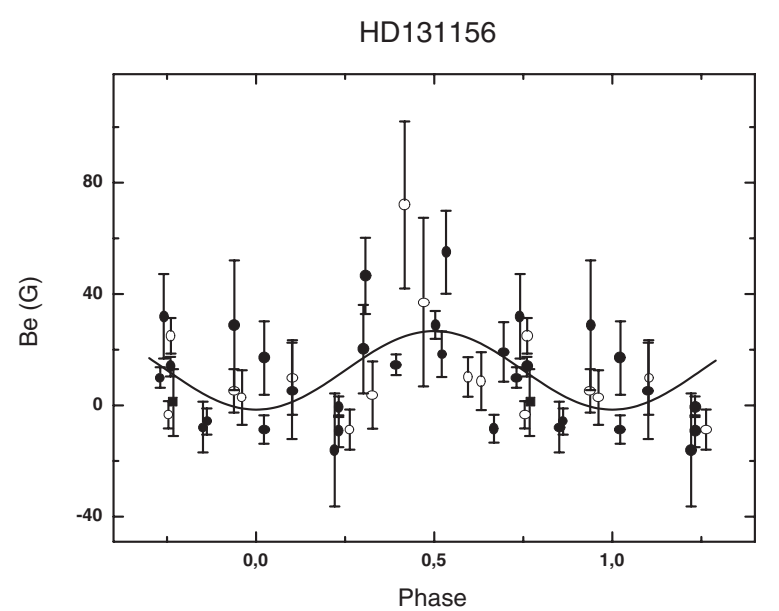

Fig. A.107. HD 131156. We used the period $P_{\mathrm{mag}}=6.1455^{\mathrm{d}}$, taken from Plachinda \& Tarasova (2000a,b). The star is an active yellow dwarf (solar type star), which displays periodic variations of $B_{\mathrm{e}}$ with rotational phase. Measurements of $B_{\mathrm{e}}$ were obtained in metal lines. Filled squares - Ref. 47, open circles - Ref. 299, filled circles Ref. 311.

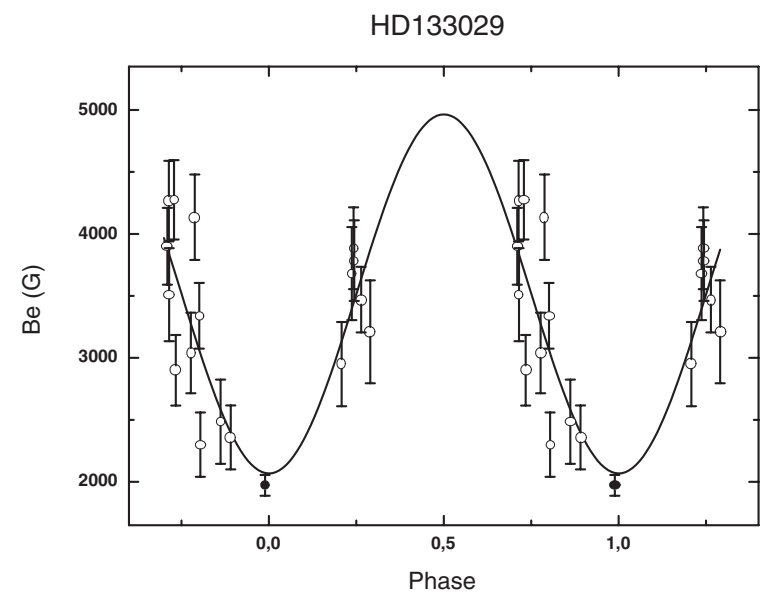

Fig. A.108. HD 133029. We have determined and used here the new magnetic period $P_{\mathrm{mag}}=2 \cdot 105^{\mathrm{d}}$. This figure displays magnetic rotational phase curve fit to $B_{\text {e }}$ points, which were measured in hydrogen lines. Open circles - Ref. 2, filled circles - Ref. 327. 
V. D. Bychkov et al.: A catalog of stellar magnetic rotational phase curves, Online Material p 36

HD133029

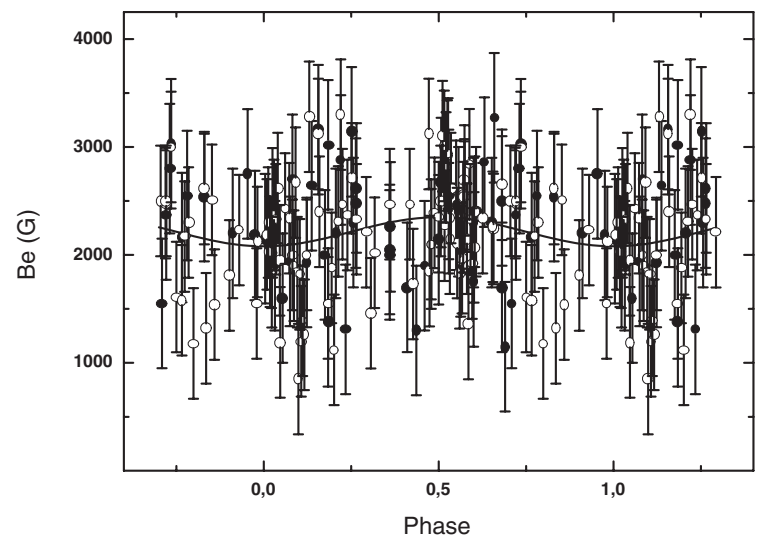

Fig. A.109. HD 133029. We determined new magnetic period $P_{\text {mag }}=2.105^{\mathrm{d}}$. Measurements of $B_{\mathrm{e}}$ from metal lines were done with the photographic method, and the corresponding phase curve is displayed here. Open circles - Ref. 94, filled circles - Ref. 1.

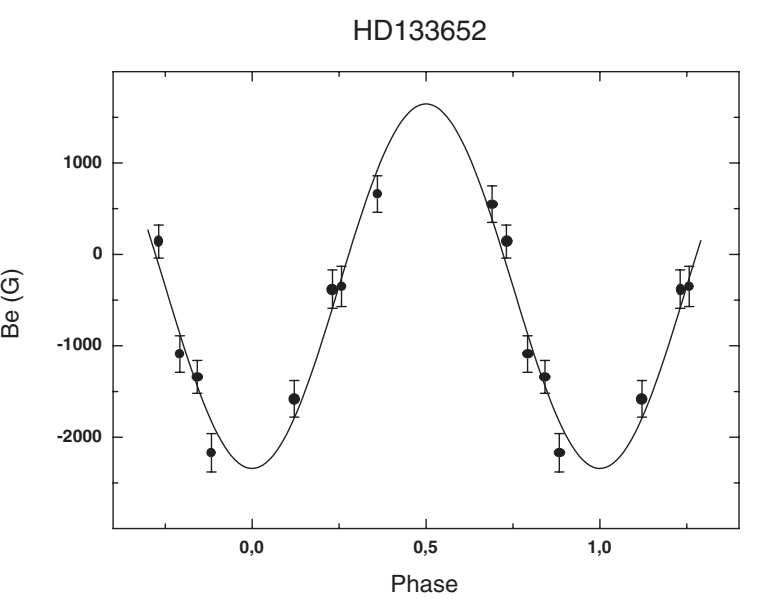

Fig. A.110. HD 133652. We used the period $P_{\mathrm{mag}}=2.3040^{\mathrm{d}}$, taken from Lanz et al. (1991). Available $B_{\mathrm{e}}$ measurements were obtained from hydrogen lines, Ref. 230. Additional observational data are necessary to obtain accurate $B_{\mathrm{e}}(\phi)$ curve.

HD133880

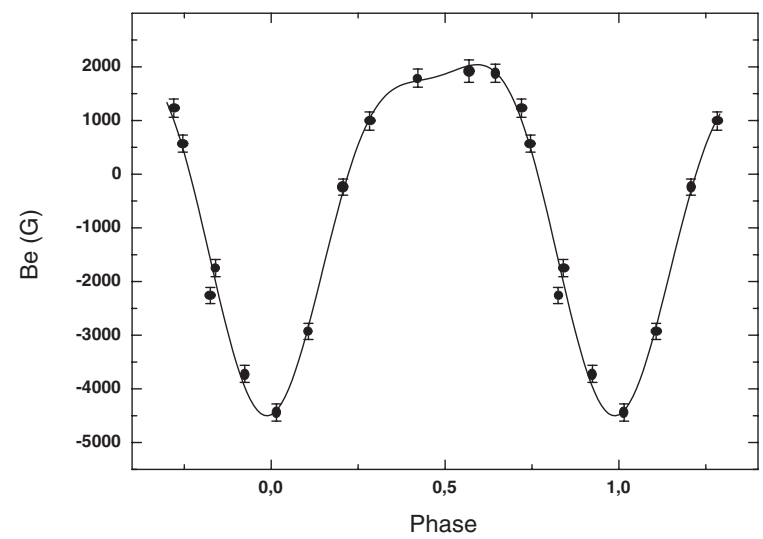

Fig. A.111. HD 133880. We used the period $P_{\text {mag }}=0.877485^{\mathrm{d}}$, taken from Landstreet (1990), Ref. 172. Magnetic field $B_{\mathrm{e}}$ was measured in hydrogen lines only - Refs. 81 and 172. Measurements from Ref. 81 were not used due to low accuracy. Phase curve $B_{\mathrm{e}}(\phi)$ is a double wave.

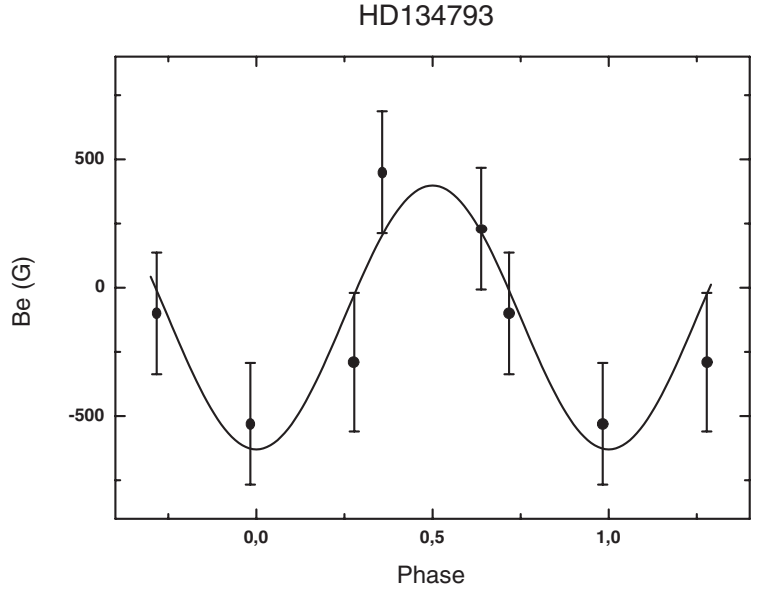

Fig. A.112. HD 134793. We used the period $P_{\text {mag }}=2.7800^{\mathrm{d}}$, taken from Blanco et al. (1978). Magnetic field $B_{\mathrm{e}}$ was measured in metal lines in Ref. 1. Additional measurements are necessary to improve parameters of magnetic variability.

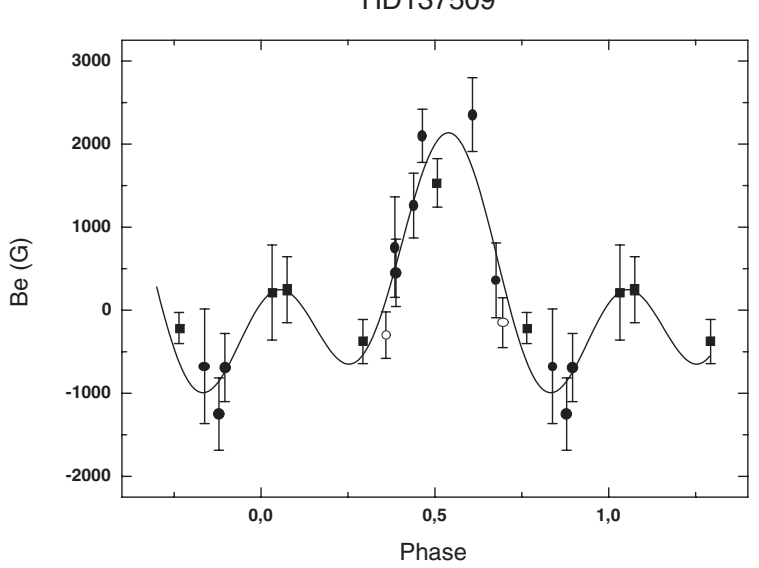

Fig. A.113. HD 137509. We used the period $P_{\mathrm{mag}}=4.4912^{\mathrm{d}}$, taken from Lanz et al. (1991). Phase curve is best described by a double wave. Filled squares - Ref. 256, open circles - Ref. 230, filled circles - Ref. 184

HD137909

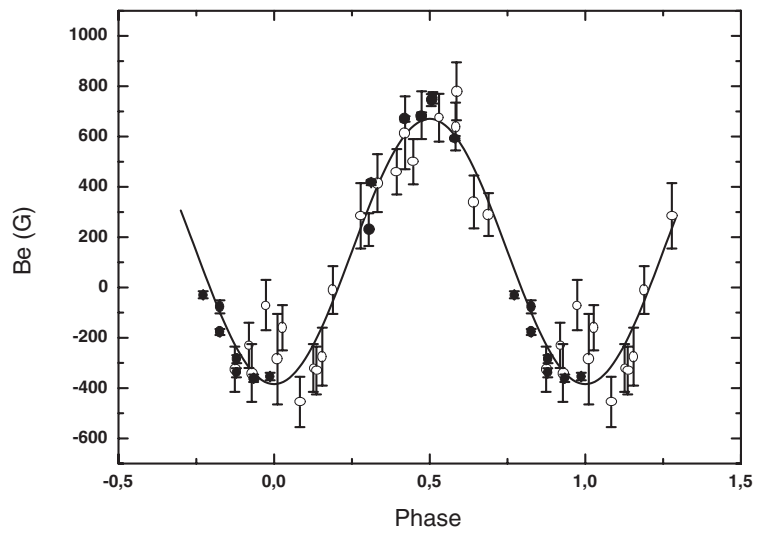

Fig. A.114. HD 137909. We used the period $P_{\mathrm{mag}}=18.4868^{\mathrm{d}}$ by Leroy (1995). Averaged hydrogen $B_{\mathrm{e}}(\phi)$ was measured in wings of $\mathrm{H}_{\beta}$ line, see Refs. 2 and 76. Magnetic $B_{\mathrm{e}}$ data, collected in Refs. 25, 77 and 190, were rejected due to low accuracy, and some of them were measured in other Balmer lines. Open circles - Ref. 2, filled circles Ref. 76. 
V. D. Bychkov et al.: A catalog of stellar magnetic rotational phase curves, Online Material p 37

HD137909

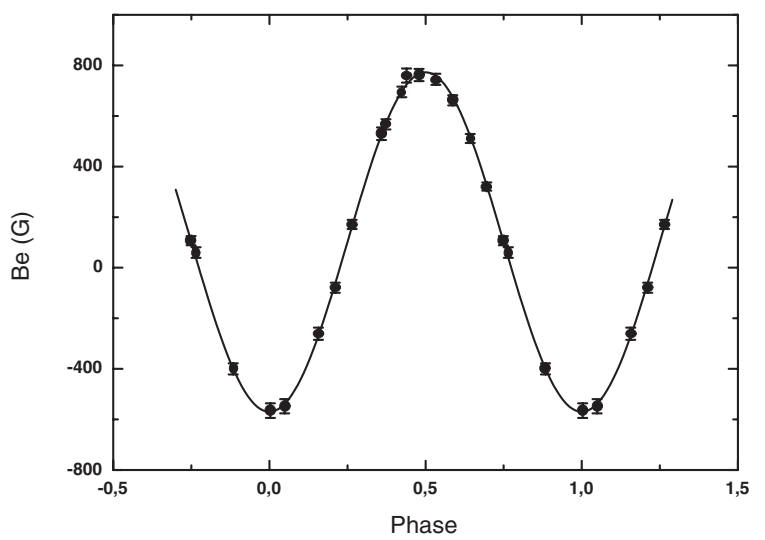

Fig. A.115. HD 137909. We used the period $P_{\mathrm{mag}}=18.4868^{\mathrm{d}}$, taken from Leroy (1995). Phase curve was obtained from $B_{\mathrm{e}}$ points determined in metal lines with the LSD method. This is one of the most accurate methods of $B_{\mathrm{e}}$ determinations.

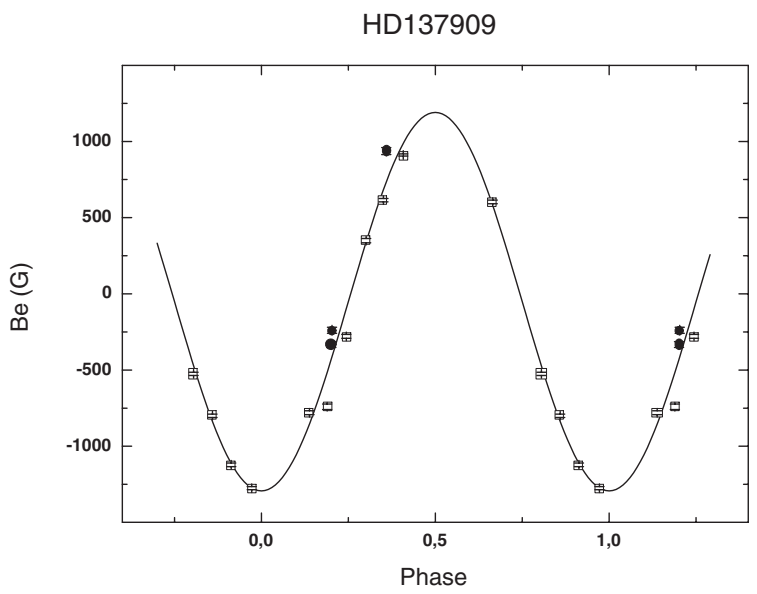

Fig. A.116. HD 137909. We used the period $P_{\mathrm{mag}}=18.4868^{\mathrm{d}}$, taken from Leroy (1995). This figure displays phase curve obtained from metal lines. The $B_{\mathrm{e}}(\phi)$ curve is very similar to that obtained by the LSD method, but the amplitude got larger. Measurements of $B_{\mathrm{e}}$ in Refs. 31 and 327 were measured in metal lines of red spectral region.

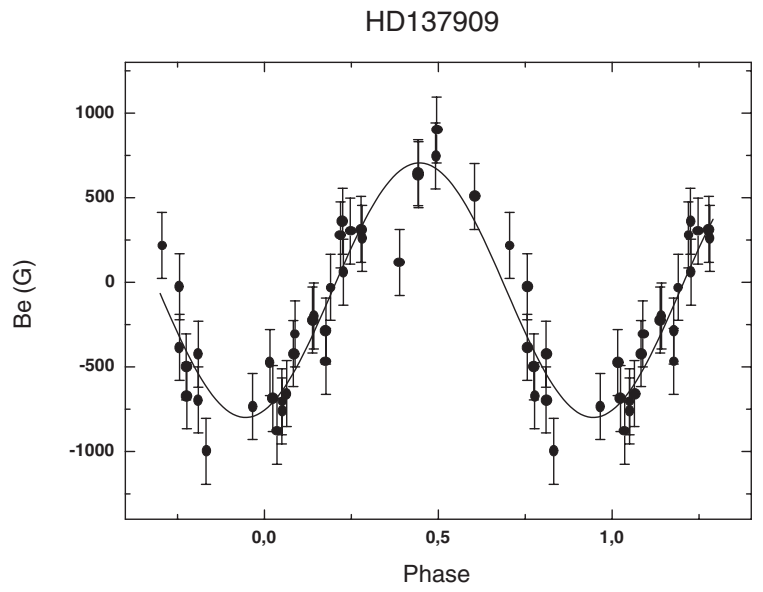

Fig. A.117. HD 137909. We used the period $P_{\mathrm{mag}}=18.4868^{\mathrm{d}}$, taken from Leroy (1995). Measurements of $B_{\mathrm{e}}$ were obtained in the Fe II 4520 line, Ref. 63. These are the most accurate $B_{\mathrm{e}}$ observations ever obtained for this star. Figure presents magnetic field data obtained by so called "integral" method.

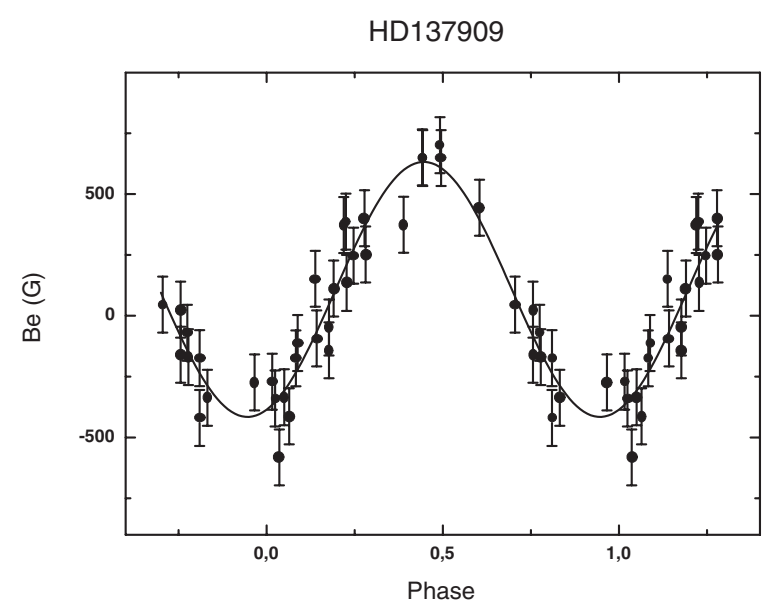

Fig. A.118. HD 137909. We used the period $P_{\mathrm{mag}}=18.4868^{\mathrm{d}}$, taken from Leroy (1995). Magnetic $B_{\mathrm{e}}$ points were measured in Fe II 4520 line by the "differential" method, see Ref. 63.

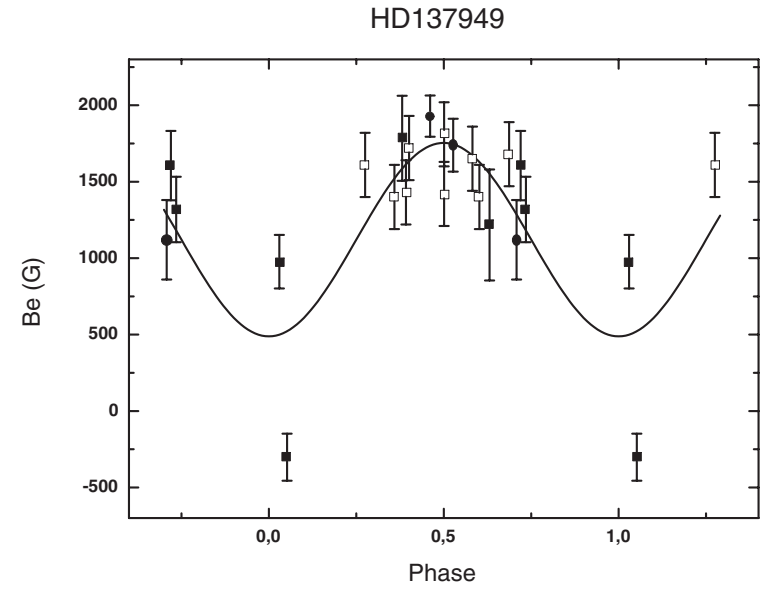

Fig. A.119. HD 137949. We determined the new probable magnetic period, $P_{\mathrm{mag}}=11.13313^{\mathrm{d}}$. This is a very interesting star, and is similar to $\gamma$ Equ in many aspects. More $B_{\mathrm{e}}$ observations are necessary to determine $P_{\text {mag }}$ and to improve other parameters of magnetic variations. Filled squares - Ref. 1, open squares - Ref. 26, open circles Ref. 91, filled circles - Ref. 256.

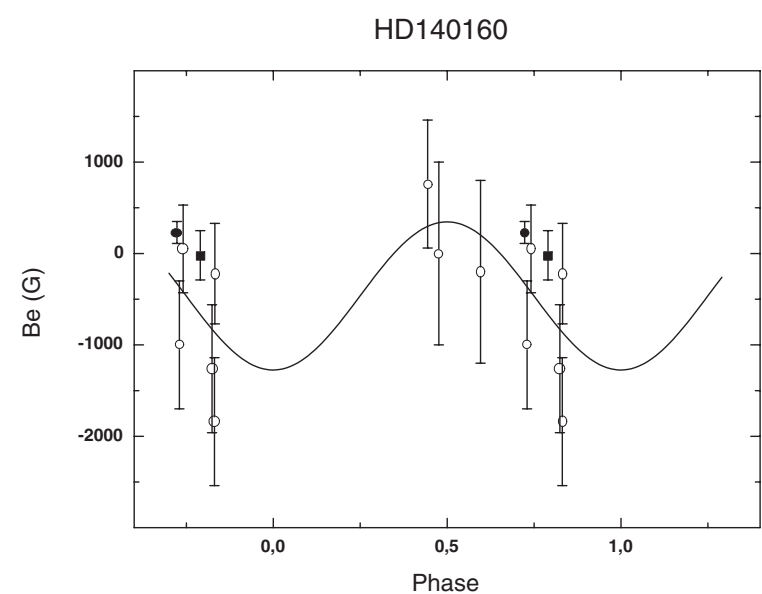

Fig. A.120. HD 140160. We used the period $P_{\text {mag }}=1.59584^{\mathrm{d}}$, taken from Musielok et al. (1980). More $B_{\mathrm{e}}$ of high accuracy are necessary for more exact description of the phase curve $B_{\mathrm{e}}(\phi)$. Filled squares Ref. 2, open circles - Ref. 25, filled circles - Ref. 333. 
V. D. Bychkov et al.: A catalog of stellar magnetic rotational phase curves, Online Material p 38

HD142301

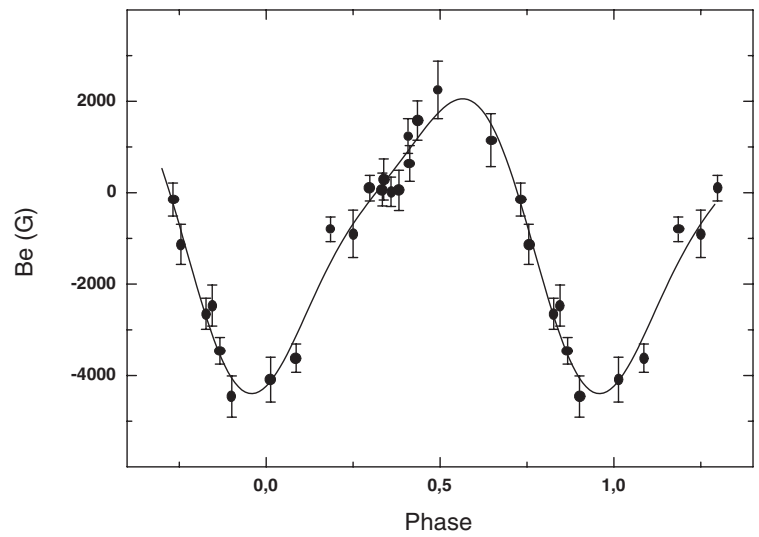

Fig. A.121. HD 142301. We used the period $P_{\mathrm{mag}}=1.45955^{\mathrm{d}}$, taken from Landstreet et al. (1979). Phase curve $B_{\mathrm{e}}(\phi)$ is best described by a double wave.

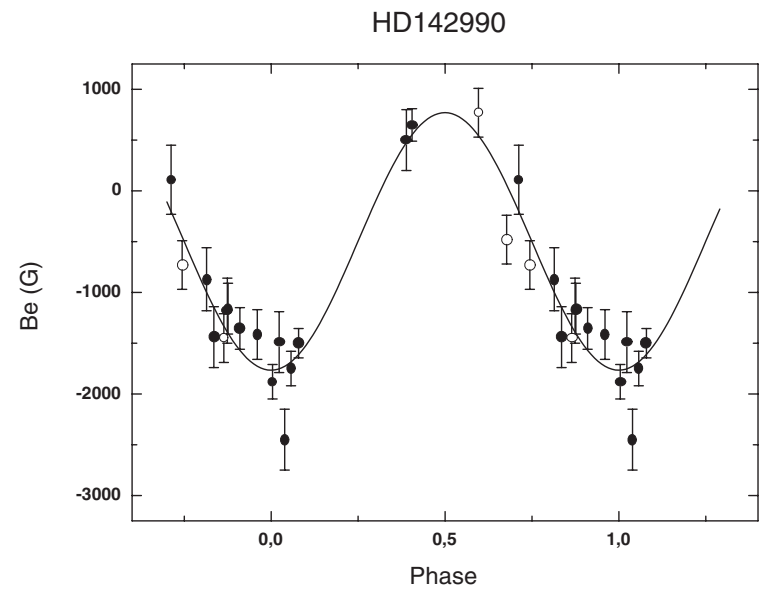

Fig. A.122. HD 142990. The best period of magnetic variations used here, $P_{\text {mag }}=0.9791^{\mathrm{d}}$. It is close to the period $P_{\mathrm{mag}}=0.979070^{\mathrm{d}}$ taken from Catalano \& Leone (1996). Magnetic field $B_{\mathrm{e}}$ was measured in hydrogen lines only. Additional $B_{\mathrm{e}}$ observations are required to improve the period $P_{\mathrm{mag}}$, and other parameters of the magnetic phase curve. Filled circles - Ref. 37, open circles - Ref. 230.

HD143473

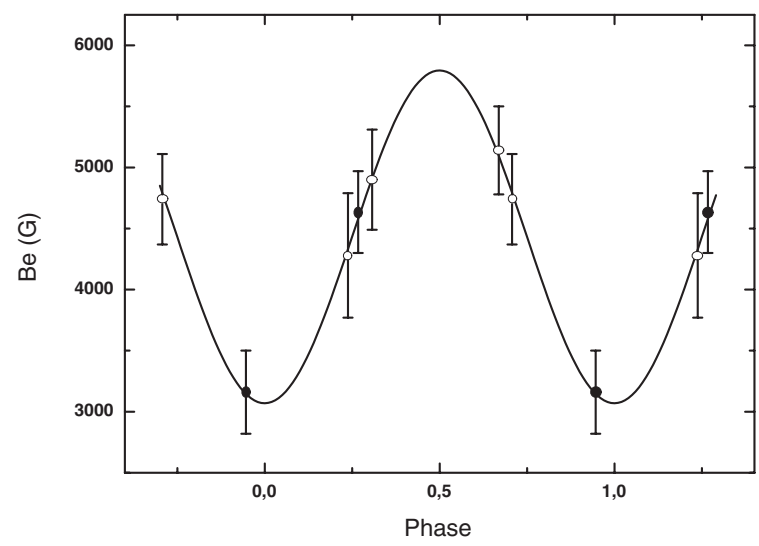

Fig. A.123. HD 143473. We determined the magnetic period, $P_{\text {mag }}=2.84263^{\mathrm{d}}$. There exists a small number of $B_{\mathrm{e}}$ measurements. Additional magnetic observations are necessary to improve the period and other parameters of the magnetic variability. Filled circles Ref. 184, open circles - Ref. 230.

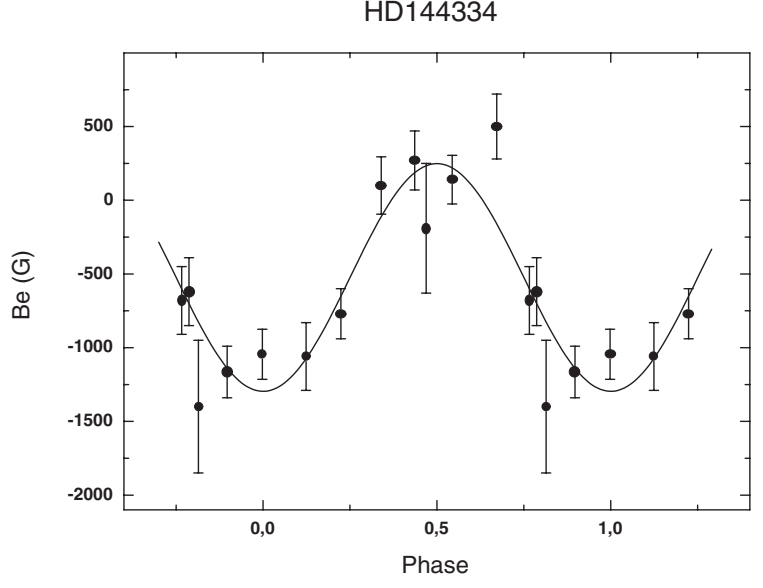

Fig. A.124. HD 144334. Phase curve $B_{\mathrm{e}}(\phi)$ was computed with the photometric period $P_{\text {phot }}=1.49497^{\mathrm{d}}$ taken from North (1987), and the magnetic period $P_{\mathrm{mag}}=3.61^{\mathrm{d}}$ (Borra et al. 1983) was not used here. There exists a small number of $B_{\mathrm{e}}$ measurements. New $B_{\mathrm{e}}$ points are necessary to improve the period and other parameters of magnetic variability.

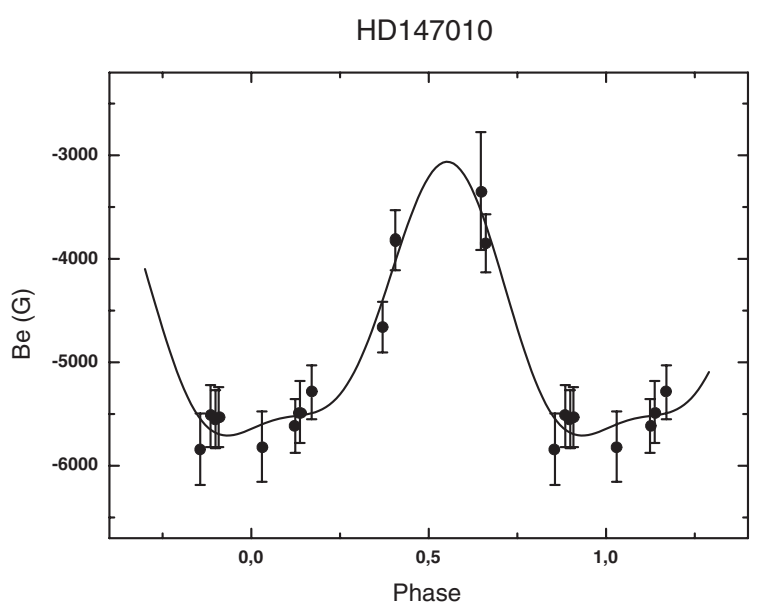

Fig. A.125. HD 147010. We used the period $P_{\text {mag }}=3.920676^{\mathrm{d}}$, taken from Catalano \& Leone (1993). Figure presents the averaged phase curve measured in hydrogen lines, and it is best represented by a double wave. 
V. D. Bychkov et al.: A catalog of stellar magnetic rotational phase curves, Online Material p 39

HD147010

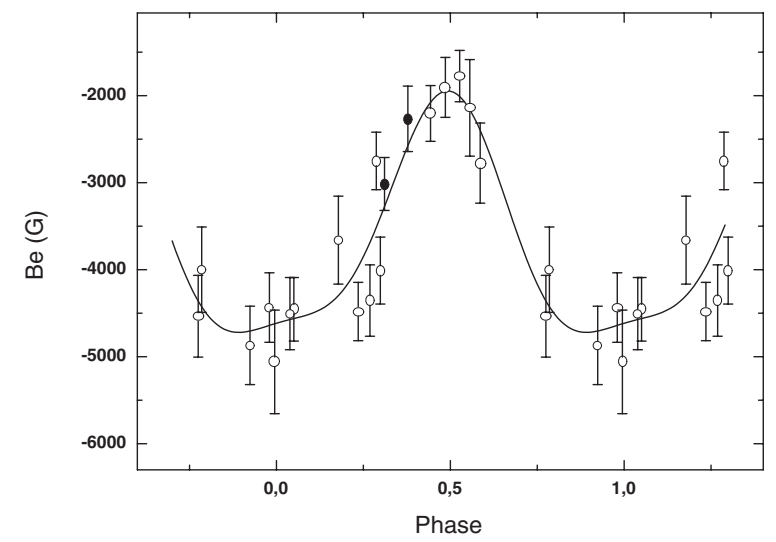

Fig. A.126. HD 147010. We used the period $P_{\text {mag }}=3.920676^{\mathrm{d}}$, taken from Catalano \& Leone (1993). Magnetic rotational phase curve was obtained from $B_{\mathrm{e}}$ observations in metal lines. It is also a double wave, but the zeroth order component of phase curve in this figure (the constant) is smaller by about $1 \mathrm{kG}$ than that in the hydrogen curve. Open circles - Ref. 184, filled circles - Ref. 256.

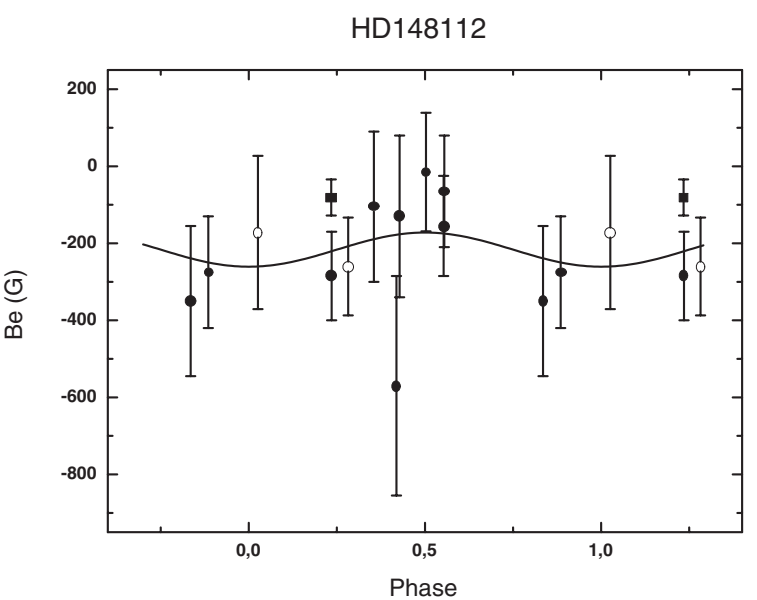

Fig. A.127. HD 148112. We used the period $P_{\mathrm{mag}}=3.04296^{\mathrm{d}}$, taken from Hatzes (1991). This star exhibits low level of magnetic variability. Averaged phase curve $B_{\mathrm{e}}(\phi)$ did not include $B_{\mathrm{e}}$ measurements from Ref. 25 due to low accuracy. New measurements of high precision are necessary to improve the period and parameters of the magnetic rotational phase curve. Filled squares - Ref. 333, open circles - Ref. 327, filled circles - Ref. 2.

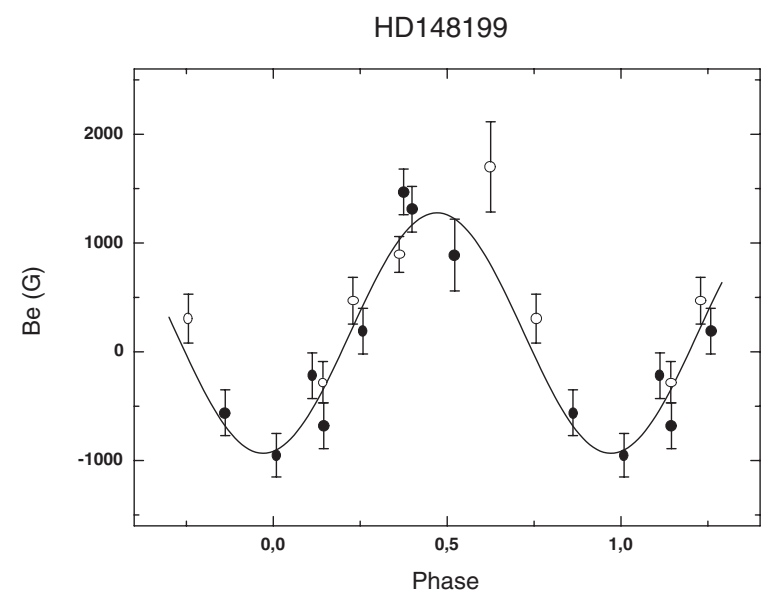

Fig. A.128. HD 148199. We used the period $P_{\mathrm{mag}}=7.72553^{\mathrm{d}}$, taken from Bohlender et al. (1993). Magnetic field $B_{\mathrm{e}}$ was determined in hydrogen lines. Open circles - Ref. 168, filled circles - Ref. 230.

HD148330

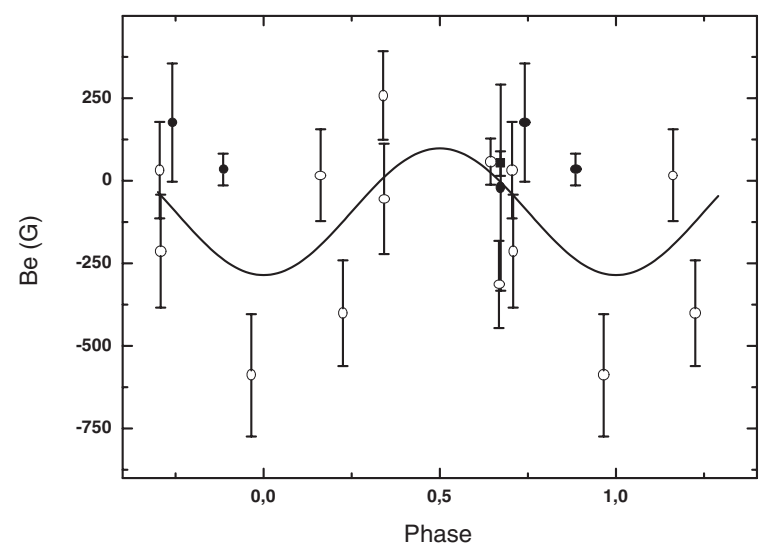

Fig. A.129. HD 148330. We used the period $P_{\text {mag }}=4.288404^{\mathrm{d}}$, Ziznovsky \& Romanyuk (1990). Magnetic behavior of this star is not well understood. More observations are necessary to determine parameters of the phase curve. Open circles - Ref. 179, filled circles Ref. 327, filled squares - Ref. 333. 
V. D. Bychkov et al.: A catalog of stellar magnetic rotational phase curves, Online Material p 40

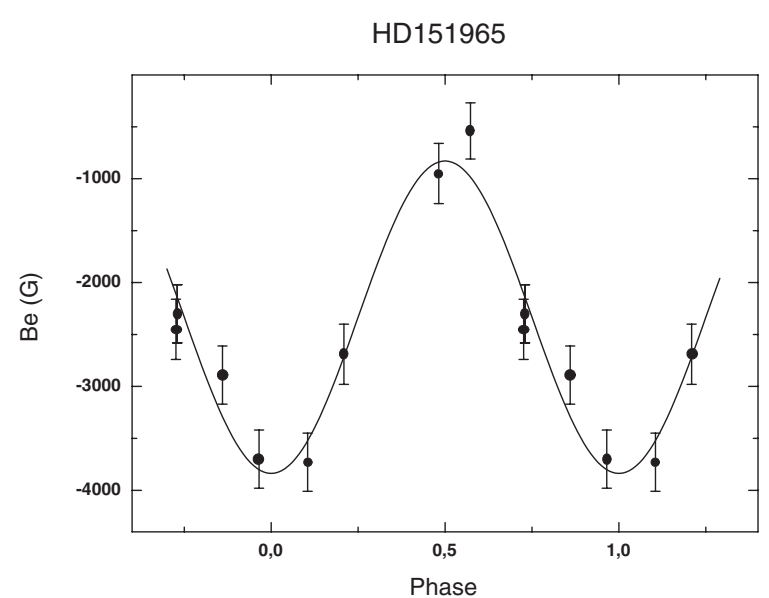

Fig. A.130. HD 151965. We used the period $P_{\mathrm{mag}}=1.60841^{\mathrm{d}}$, taken from Lanz \& Mathys (1991b). Magnetic field $B_{\mathrm{e}}$ was observed in hydrogen lines - Ref. 230.

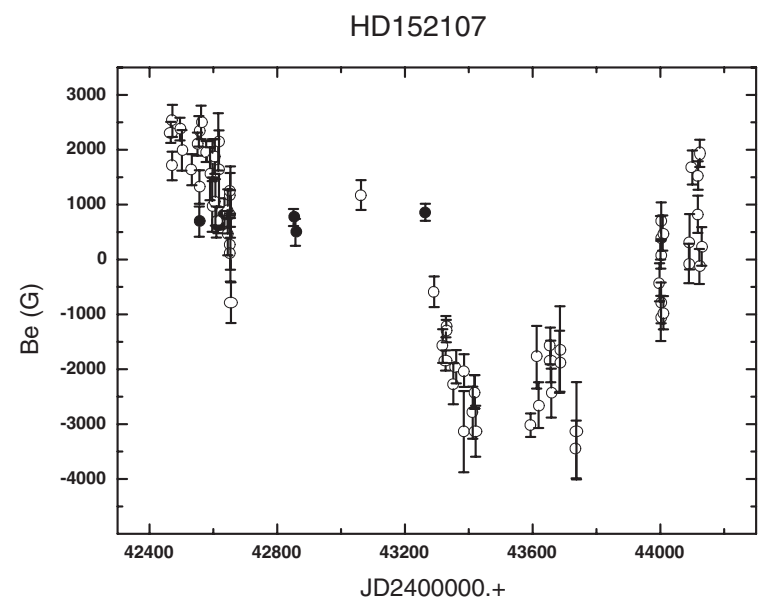

Fig. A.131. HD 152107. Figure presents magnetic behavior of this star in a long time scale.

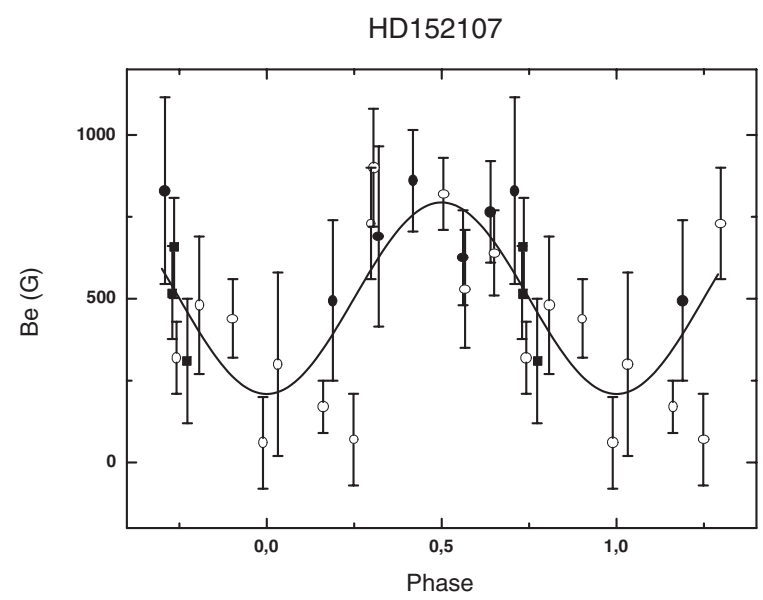

Fig. A.132. HD 152107. We determined and used here the period $P_{\text {mag }}=3.86778^{\mathrm{d}}$. It is close to the period $P_{\text {mag }}=3.85646^{\mathrm{d}}$, taken from Gerth (1990b). The average phase curve $B_{\mathrm{e}}(\phi)$ was obtained from hydrogen lines. Open circles - Ref. 220, filled squares - Ref. 290, filled circles - Ref. 2 .

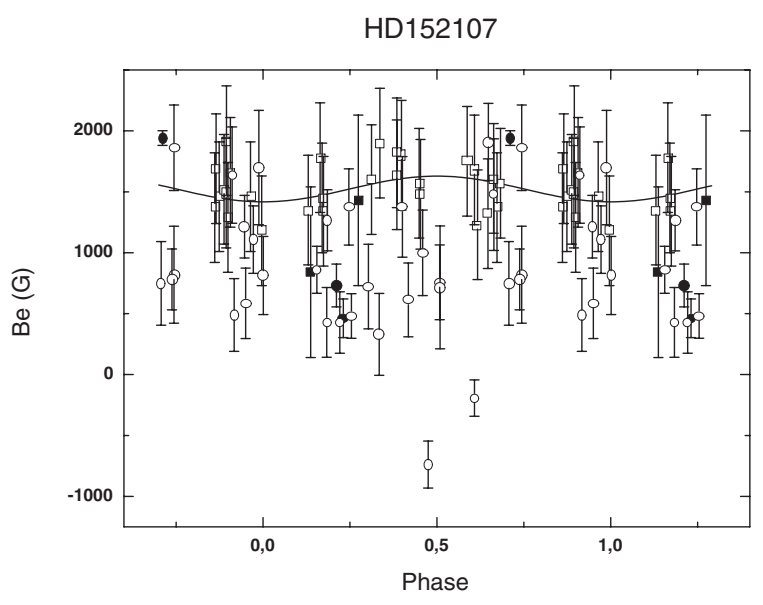

Fig. A.133. HD 152107. We determined and applied here the period $P_{\text {mag }}=3.86778^{\mathrm{d}}$. It is close to the period $P_{\mathrm{mag}}=3.86778^{\mathrm{d}}$ by Gerth (1990b). The phase curve was obtained from $B_{\mathrm{e}}$ measurements in metal lines. The scatter of $B_{\mathrm{e}}$ points is very large. The phase curve should be improved by the additional high accuracy observations. Open squares - Ref. 1, open circles - Refs. 178, 219 and 290, filled circles - Ref. 327.

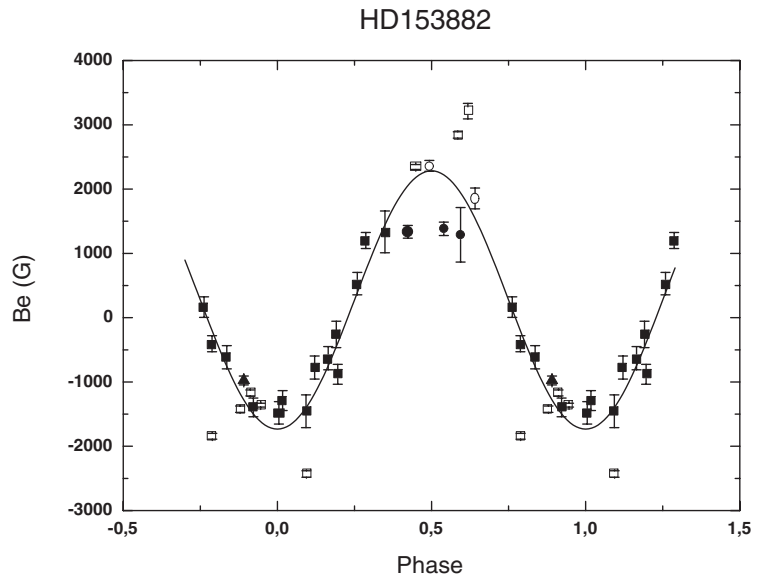

Fig. A.134. HD 153882. The best magnetic period used here, $P_{\text {mag }}=6.00858^{\mathrm{d}}$. It is close to the period $P_{\mathrm{mag}}=6.00890^{\mathrm{d}}$, taken from Mathys (1991). Filled squares - Ref. 184, open squares - Ref. 31, open circles - Ref. 72, filled circles - Ref. 256, filled triangles - Ref. 310. 
V. D. Bychkov et al.: A catalog of stellar magnetic rotational phase curves, Online Material p 41

HD164258

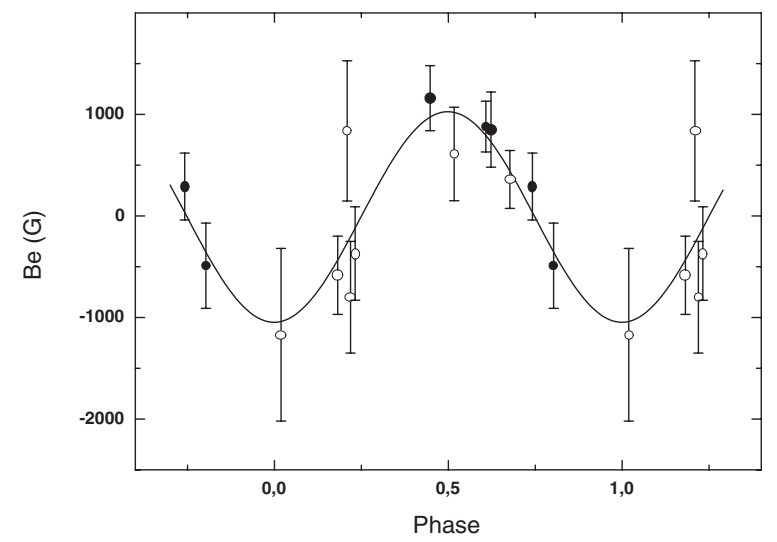

Fig. A.135. HD 164258. Magnetic field of this star was measured in metal lines with photographic method. The best period of magnetic variations used here, $P_{\mathrm{mag}}=0.86281^{\mathrm{d}}$. It is close to the magnetic period $P_{\text {mag }}=0.829^{\mathrm{d}}$, taken from Catalano \& Leone (1994). Additional high precision $B_{\mathrm{e}}$ observations are necessary for more precise determination of $P_{\mathrm{mag}}$ and parameters of magnetic variability. Filled squares Ref. 98, open squares - Ref. 327, Open squares - Ref. 41, open circles - Ref. 327.

\section{HD168733}

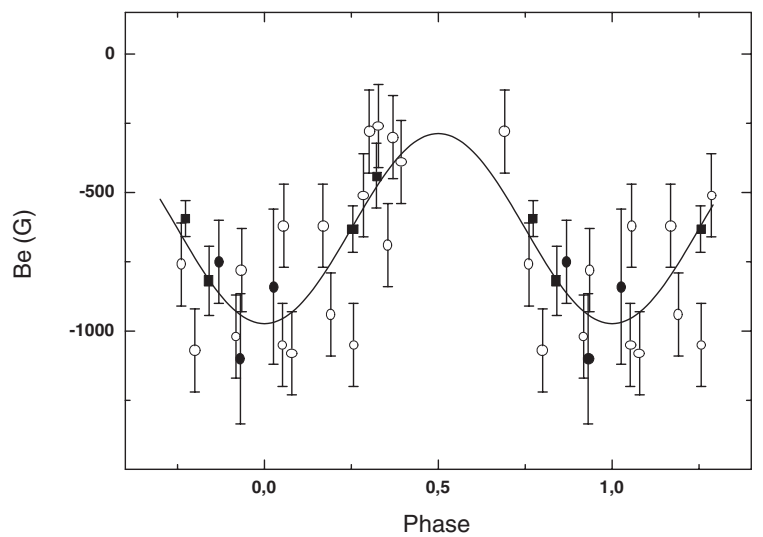

Fig. A.136. HD 168733. The best magnetic period used here, $P_{\mathrm{mag}}=14.78437^{\mathrm{d}}$. It is close to the magnetic period $P_{\mathrm{mag}}=14.6^{\mathrm{d}}$, determined by Jones \& Wolff (1974). Magnetic field $B_{\mathrm{e}}$ was measured in metal lines. Measurements from Ref. 93 were not used due to low accuracy. Additional high accuracy measurements are necessary to improve period and parameters of magnetic variability. Filled squares Ref. 256, open circles - Ref. 86, filled circles - Ref. 184.

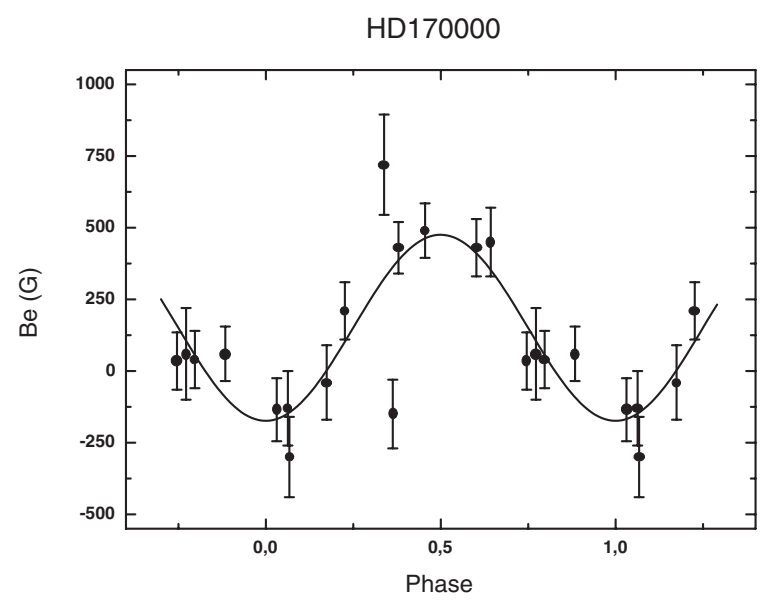

Fig. A.137. HD 170000. We used the period $P_{\text {mag }}=1.71649^{\mathrm{d}}$, taken from Musielok \& Madej (1988). Magnetic field points $B_{\mathrm{e}}$ were measured in $\mathrm{H}_{\beta}$ line - Ref. 60 . This is the most accurate $B_{\mathrm{e}}(\phi)$ phase curve available for this star.

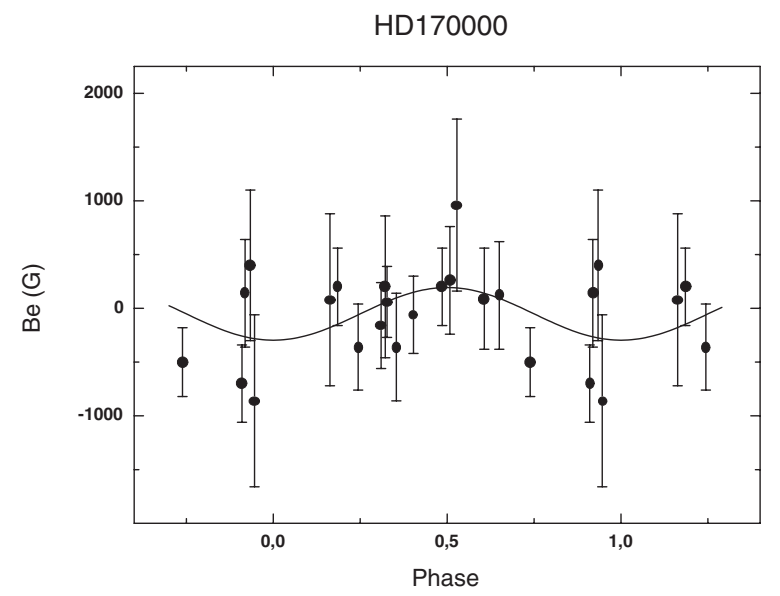

Fig. A.138. HD 170000. We used the period $P_{\mathrm{mag}}=1.71649^{\mathrm{d}}$, taken from Musielok \& Madej (1988). Phase curve $B_{\mathrm{e}}(\phi)$ was measured in $\mathrm{H}_{\alpha}$ line - Ref. 25. Note that this phase curve remains in antiphase as compared to phase curve in the previous figure, i.e. points $\phi=0$ differ by $0.5 \times P_{\text {mag }}$. 
V. D. Bychkov et al.: A catalog of stellar magnetic rotational phase curves, Online Material p 42

HD170397

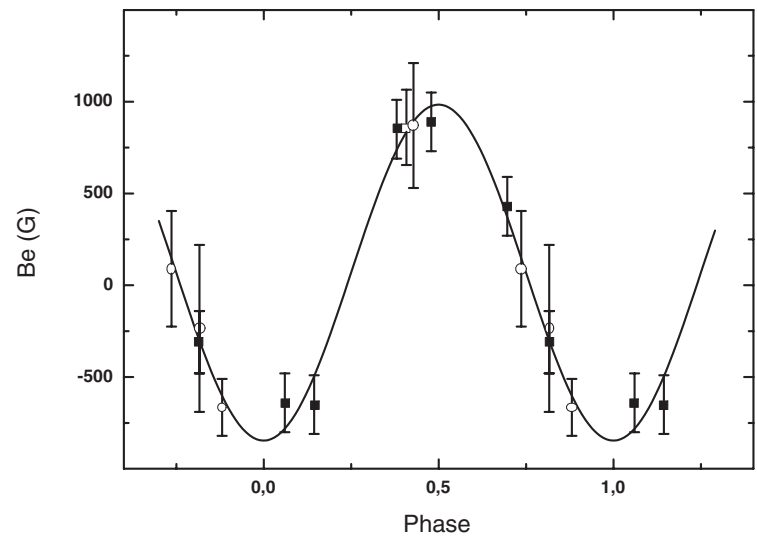

Fig. A.139. HD 170397. The best period of magnetic variability used here, $P_{\text {mag }}=2.25454^{\mathrm{d}}$. It is close to the magnetic period $P_{\mathrm{mag}}=2.24^{\mathrm{d}}$, taken from Borra \& Landstreet (1980). More $B_{\mathrm{e}}$ measurements are necessary to improve the period and other parameters of phase curve. Open circles - Ref. 2, open squares - Ref. 184, filled squares Ref. 230.

HD170973

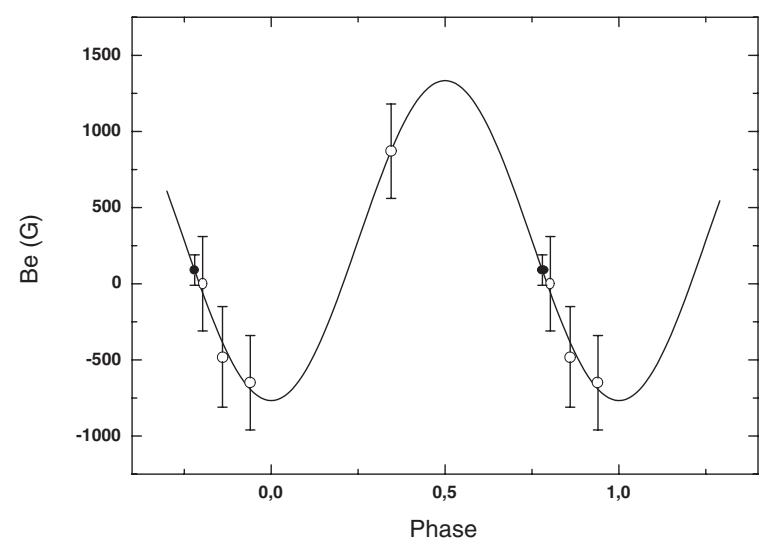

Fig. A.140. HD 170973. The best period of magnetic variability, used here, $P_{\text {mag }}=18.52^{\mathrm{d}}$. It is close to the magnetic period $P_{\mathrm{mag}}=18.2^{\mathrm{d}}$, taken from Bohlender et al. (1993). Available $B_{\mathrm{e}}$ measurements are very scarce. New measurements of high precision are necessary to improve the period and parameters of the magnetic rotational phase curve. Open circles - Ref. 230, filled circles - Ref. 184.

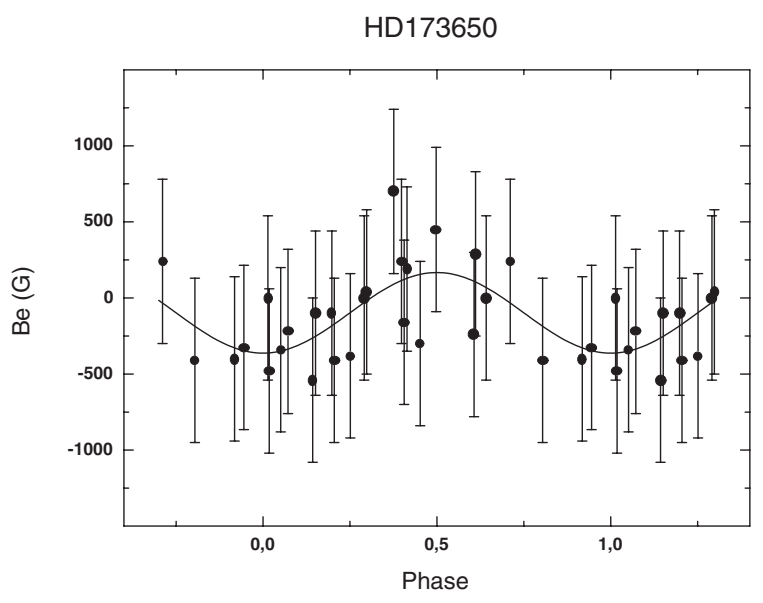

Fig. A.141. HD 173650. We used the period $P_{\mathrm{mag}}=9.9754^{\mathrm{d}}$, taken from Hildebrandt et al. (1985). Magnetic points $B_{\mathrm{e}}$ were measured in metal lines with the photographic technique, see Ref. 1. Additional high accuracy measurements are necessary to improve parameters of the magnetic variability of this star.

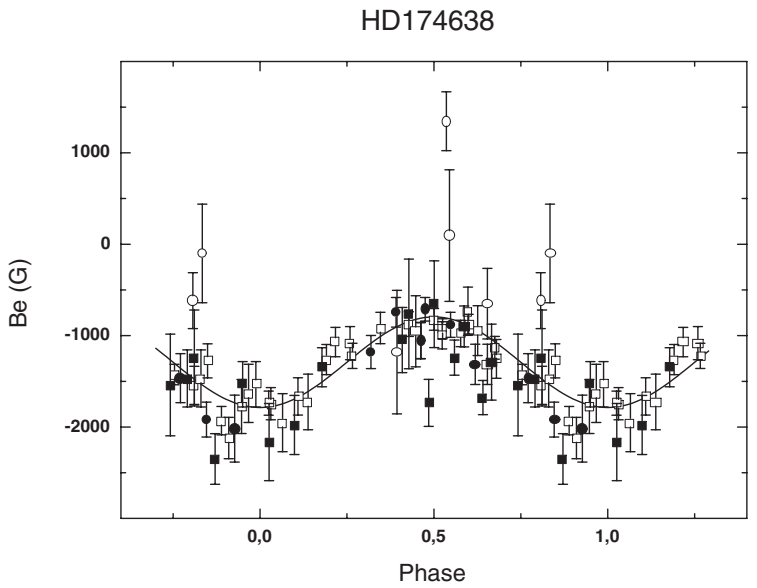

Fig. A.142. HD $174638=\beta$ Lyr. We used the period $P_{\mathrm{mag}}=12.935^{\mathrm{d}}$, taken from Skulskij (1982). Measurements of $B_{\mathrm{e}}$ included in this figure were performed mostly in metal lines of blue optical region, with the photographic method. Filled squares - Ref. 342, open squares Ref. 191, open circles - Ref. 227, filled circles - Ref. 343. 
V. D. Bychkov et al.: A catalog of stellar magnetic rotational phase curves, Online Material p 43

HD174638

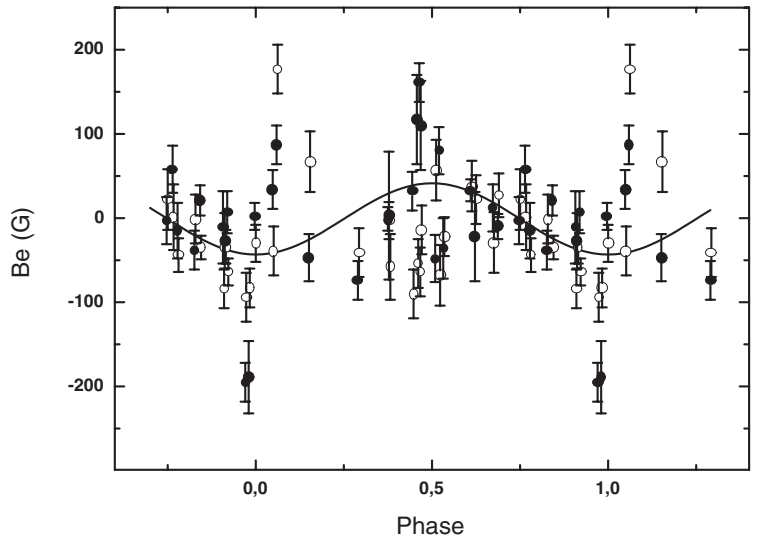

Fig. A.143. HD $174638=\beta$ Lyr. We used the period $P_{\text {mag }}=12.935^{\mathrm{d}}$, taken from Skulskij (1982). Measurements of $B_{\mathrm{e}}$ in this figure were done in red lines of silicon, and show lower level of $B_{\mathrm{e}}$ variability than these in Fig. A.142. Open circles - line SiII 6347, filled circles SiII 6371.

HD175362

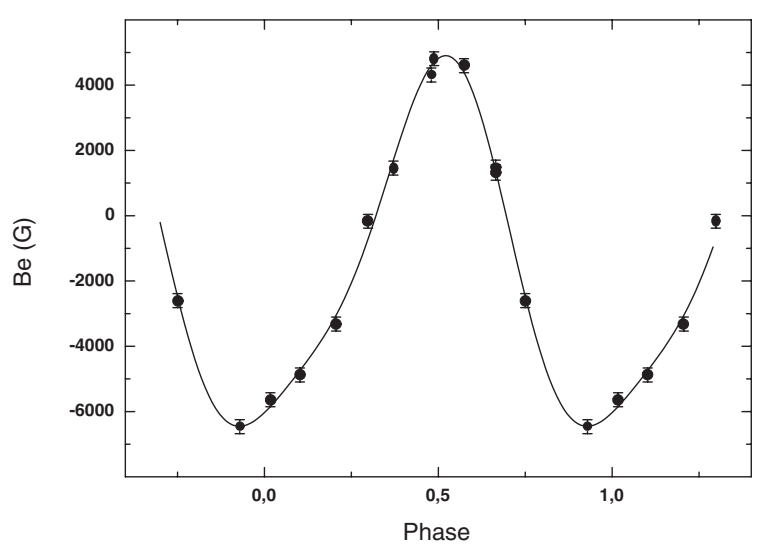

Fig. A.144. HD 175362. We used the period $P_{\text {mag }}=3.67375^{\mathrm{d}}$, taken from Catalano \& Leone (1996). Phase curve $B_{\mathrm{e}}(\phi)$ is described by a double wave. $B_{\mathrm{e}}$ points were determined in hydrogen lines - Ref. 37 .

HD175362

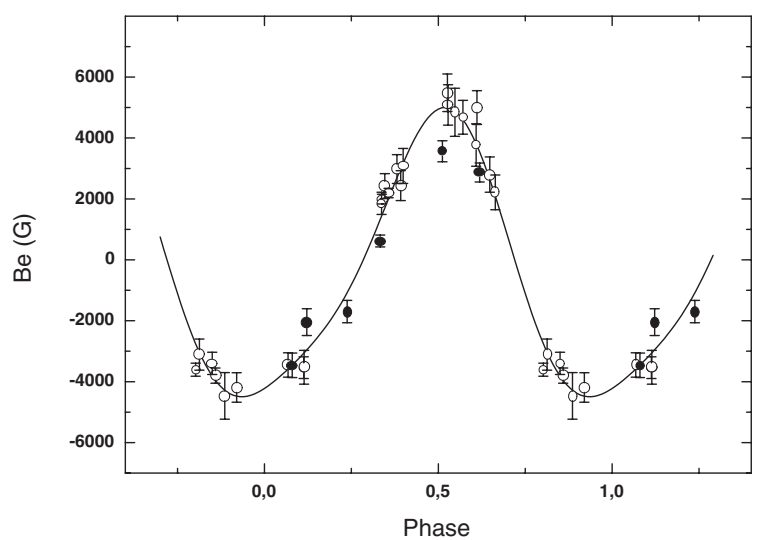

Fig. A.145. HD 175362. We used the period $P_{\mathrm{mag}}=3.67375^{\mathrm{d}}$, taken from Catalano \& Leone (1996). Magnetic $B_{\mathrm{e}}(\phi)$ phase curve was obtained from photographic $B_{\mathrm{e}}$ data. Points of Ref. 52 were rejected due to low accuracy. Open circles - Ref. 184, filled circles - Ref. 256.

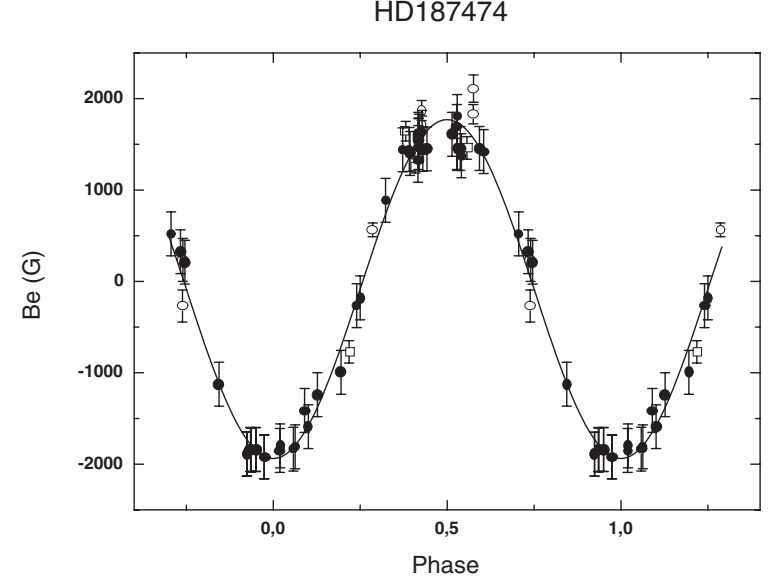

Fig. A.146. HD 187474. We determined and used the period $P_{\mathrm{mag}}=2342^{\mathrm{d}}$. It is close to the period $P_{\mathrm{mag}}=2345^{\mathrm{d}}$, taken from Hensberge (1993). Magnetic field $B_{\mathrm{e}}$ was measured in metal lines only. Open squares - Ref. 256, open circles - Ref. 184, filled circles Refs. 1 and 184 - Babcock's measurements.

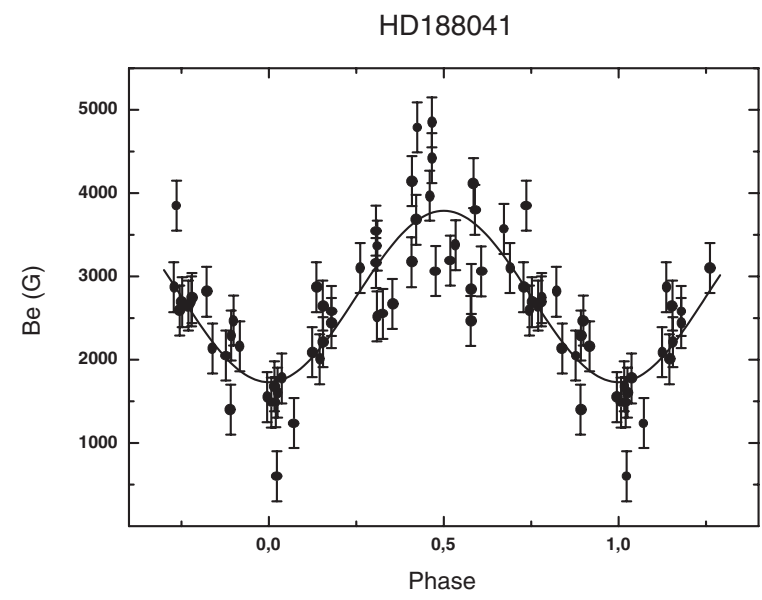

Fig. A.147. HD 188041. We determined and used here the period $P_{\mathrm{mag}}=223.826^{\mathrm{d}}$. It is very close to the period $P_{\mathrm{mag}}=223.9^{\mathrm{d}}$, taken from Hensberge (1993). Phase curve $B_{\mathrm{e}}(\phi)$ was obtained from $B_{\mathrm{e}}$ points presented in Ref. 4 (measurements in metal lines). 
V. D. Bychkov et al.: A catalog of stellar magnetic rotational phase curves, Online Material p 44

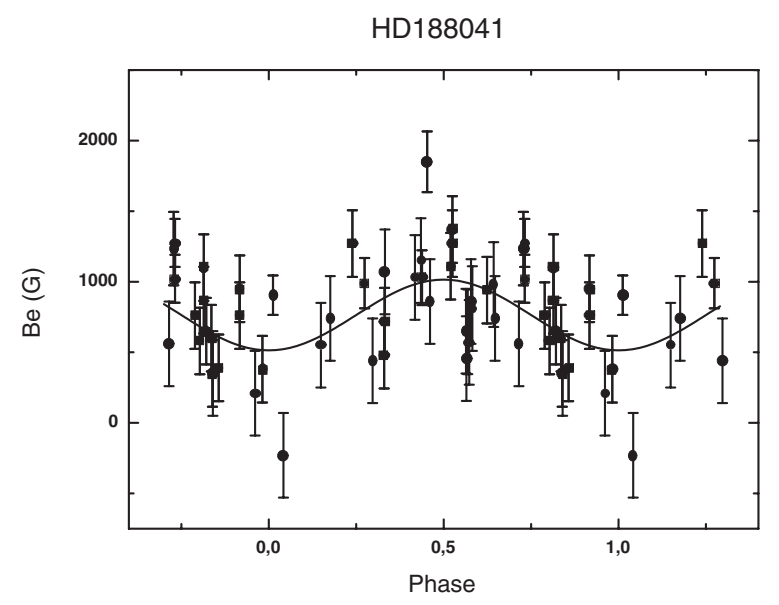

Fig. A.148. HD 188041. We determined and used here the period $P_{\text {mag }}=223.826^{\mathrm{d}}$. It is very close to the period $P_{\mathrm{mag}}=223.9^{\mathrm{d}}$, taken from Hensberge (1993). Phase curve was derived from $B_{\mathrm{e}}$ measurements published in Refs. 1, 15, 16, 184 and 256. $B_{\mathrm{e}}$ data in Ref. 232 were not used here, because they strongly differ between themselves. Filled squares - Refs. 15 and 16, open squares - Ref. 256, open circles - Ref. 184, filled circles - Ref. 1.

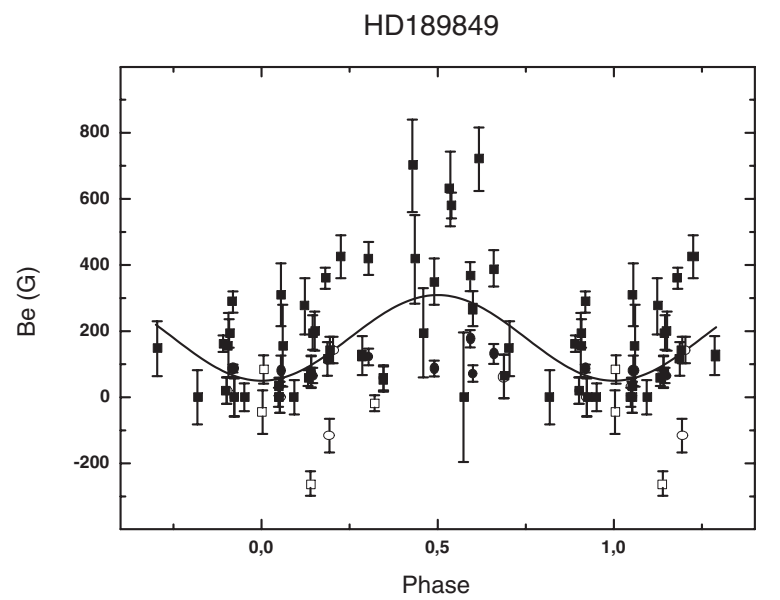

Fig. A.149. HD 189849. Magnetic field $B_{\mathrm{e}}$ was measured in metal lines. We determined and used here the period $P_{\mathrm{mag}}=6.88449^{\mathrm{d}}$. It is in the range $P_{\text {mag }}=4-6^{\mathrm{d}}$, determined by Kuvshinov (1972), Ref. 97. More $B_{\mathrm{e}}$ points are necessary here. Filled squares - Ref. 98, open squares - Ref. 327, open circles - Ref. 10, filled circles - Ref. 97.

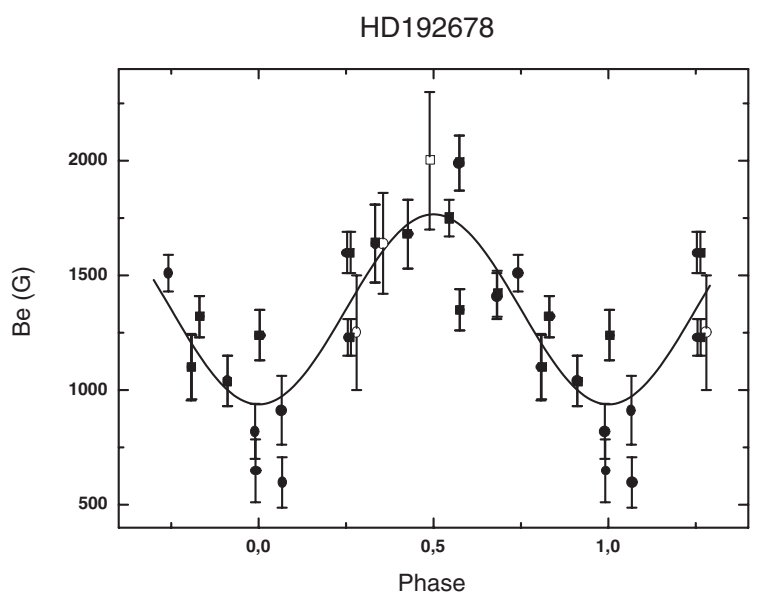

Fig. A.150. HD 192678. We used here one of the probable magnetic periods, $P_{\mathrm{mag}}=12.91049^{\mathrm{d}}$. It is over twice larger than the period $P_{\mathrm{mag}}=6.4186^{\mathrm{d}}$, given by Wade et al. (1996). Additional $B_{\mathrm{e}}$ measurements of high precision are necessary for the unique determination of the period $P_{\mathrm{mag}}$ and phase curve $B_{\mathrm{e}}(\phi)$. Filled squares - Ref. 41, open squares - Ref. 1, open circles - Ref. $255\left(\mathrm{H}_{\beta}\right.$ line $)$, filled circles Ref. 255 (metal lines).

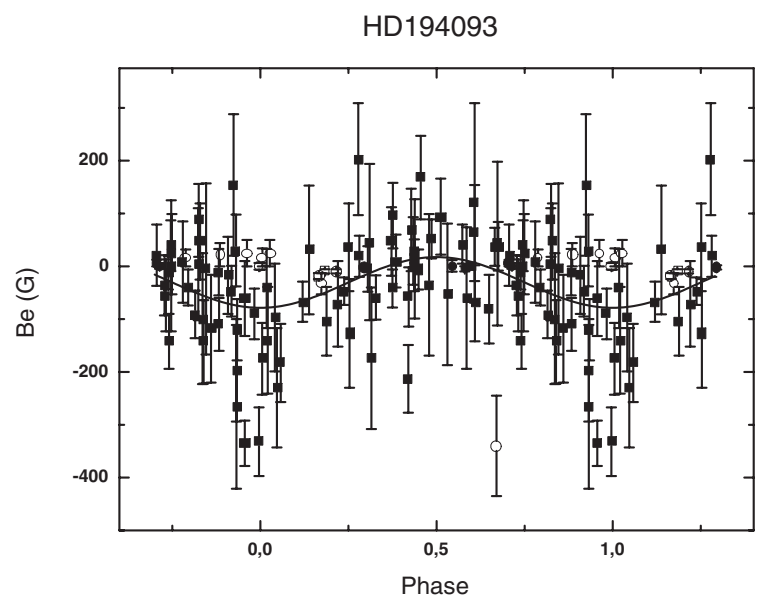

Fig. A.151. HD 194093. We used the period $P_{\mathrm{mag}}=1.03474^{\mathrm{d}}$, taken from Plachinda (1990), Ref. 189. Magnetic field $B_{\mathrm{e}}$ was determined from metal lines. Measurements in Refs. 56 and 131 were not used here due to low accuracy. Open squares - Ref. 76, open circles Ref. 140, filled squares - Ref. 189, filled circles - Ref. 299. 
V. D. Bychkov et al.: A catalog of stellar magnetic rotational phase curves, Online Material p 45

HD196178

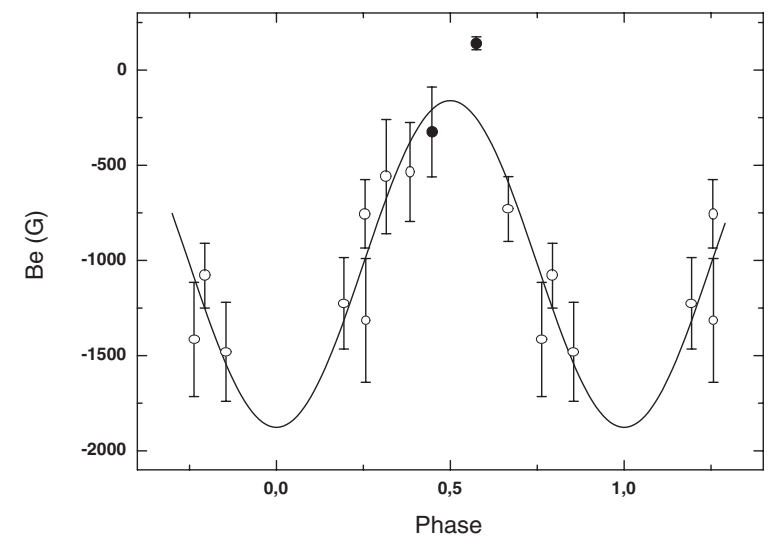

Fig. A.152. HD 196178. We determined and used here the period $P_{\text {mag }}=1.91645^{\mathrm{d}}$. The number of available $B_{\mathrm{e}}$ observations is small. The unique determination of the period and other parameters of the phase curve requires additional high precision $B_{\mathrm{e}}$ observations. Open circles - Ref. 2, filled circles - Ref. 327.

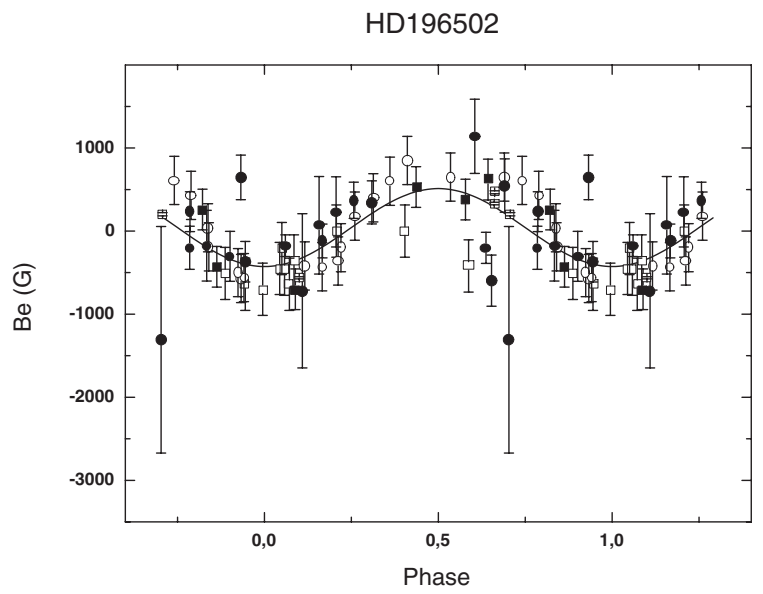

Fig. A.153. HD 196502. We used the period $P_{\text {mag }}=20.2747^{\mathrm{d}}$, taken from Schoneich et al. (1976). Magnetic field of this star was measured using various techniques. Open squares - Ref. 1, open circles Ref. 7, filled squares - Ref. 22, filled circles - Ref. 190, open triangles - Ref. 327.
HD201091

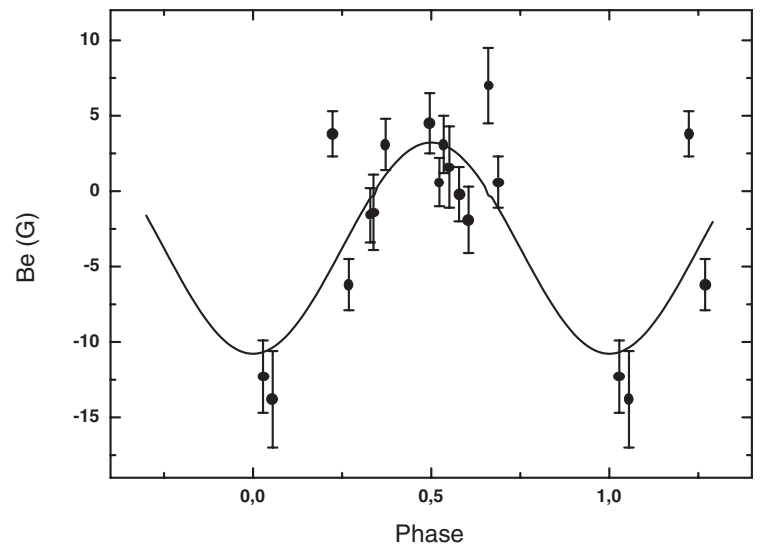

Fig. A.154. HD 201091. We used the period $P_{\mathrm{mag}}=36.59^{\mathrm{d}}$, taken from Tarasova et al. (2001), Ref. 354. The effective magnetic field $B_{\mathrm{e}}$ was measured in metal lines. The above phase curve was constructed on $B_{\text {e }}$ points taken only from Ref. 354 . Measurements from Refs. 47, 70 and 299 , were not used due to low accuracy.

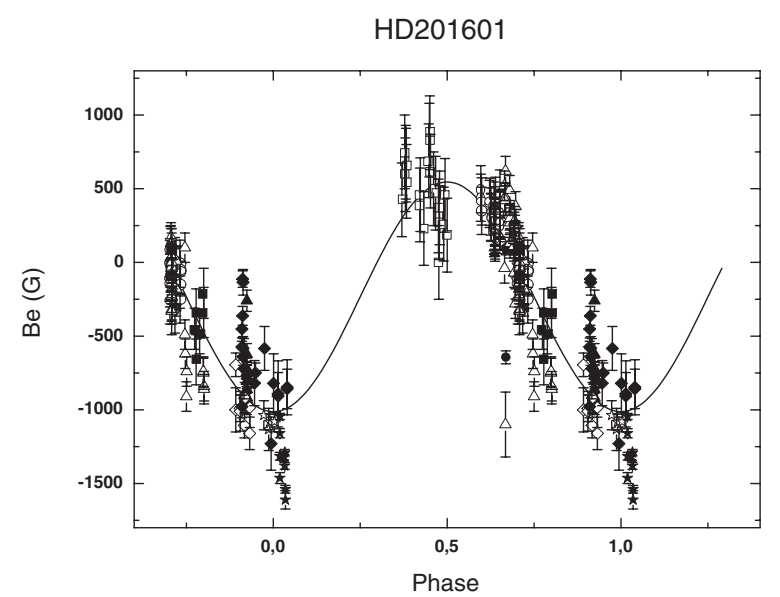

Fig. A.155. HD 201601. We have found the period $P_{\mathrm{mag}}=27027^{\mathrm{d}}$. Open squares - Ref. 1, filled squares - Ref. 46, open circles - Ref. 2, filled circles - Ref. 47, open triangles - Refs. 48, 49 and 105, filled triangles - Ref. 146, open diamonds - Ref. 184, filled diamonds - Refs. 195 and 268, open asterisks - Ref. 256, filled asterisks Ref. 327. 
V. D. Bychkov et al.: A catalog of stellar magnetic rotational phase curves, Online Material p 46

HD207260

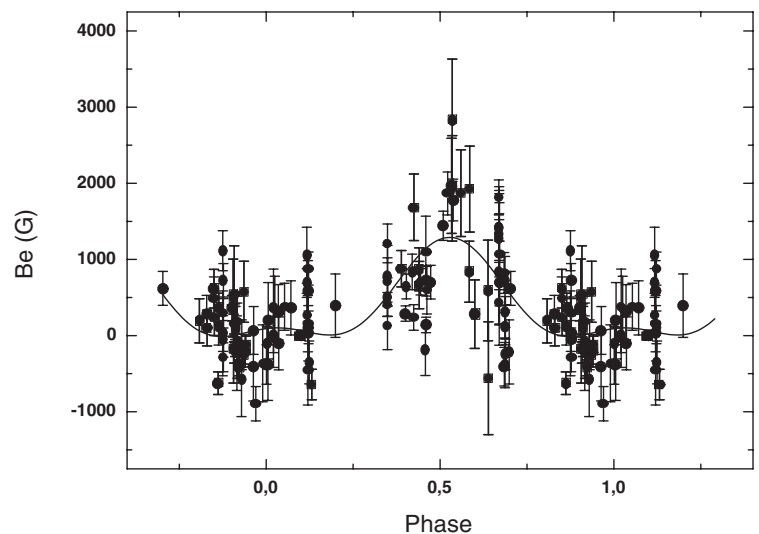

Fig. A.156. HD 207260. We have found the most probable period $P_{\text {mag }}=1688.9^{\text {d }}$. Phase curve $B_{\mathrm{e}}(\phi)$ is described best by a double wave. New $B_{\mathrm{e}}$ high precision measurements are necessary to the unique determination of $P_{\mathrm{mag}}$ and other parameters of the magnetic variability. Open squares - Ref. 136, filled squares - Ref. 325, open circles Ref. 194.

HD215441

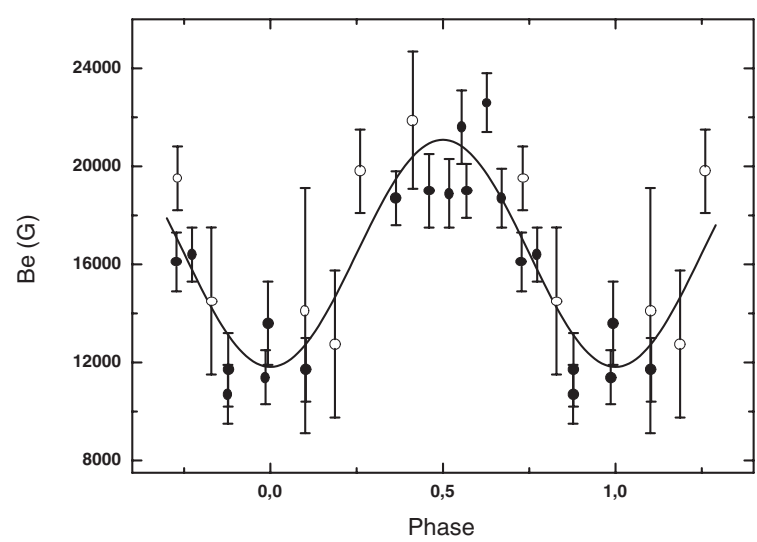

Fig. A.157. HD 215441. Measurements of $B_{\mathrm{e}}$ in hydrogen lines exhibit periodic variability with the rotational period $P_{\text {rot }}=9.4871^{\mathrm{d}}$ taken from Leckrone (1974). Filled circles - Ref. 30, open circles - Ref. 190.

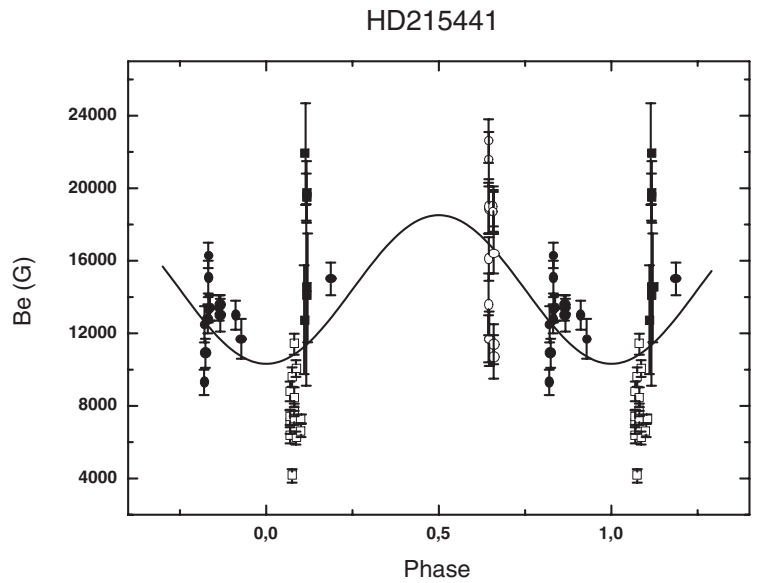

Fig. A.158. HD 215441. We determined that the variability with the large period $P_{\text {long }}=10180^{\mathrm{d}}$ cannot be ruled out. The figure shows $B_{\mathrm{e}}(\phi)$ curve plotted using this period. Open squares - Ref. 5, filled squares - Ref. 190, open circles - Ref. 30, filled circles Ref. 224.

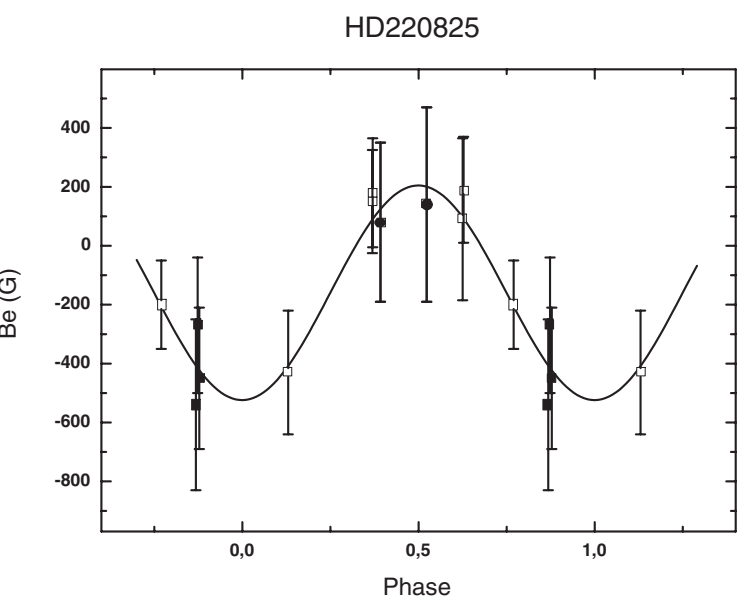

Fig. A.159. HD 220825. We determined and used here the period $P_{\text {mag }}=1.14077^{\mathrm{d}}$. It is very close to the period $P=1.412^{\mathrm{d}}$, taken from Kerschbaum \& Maitzen (1991). The number of available $B_{\mathrm{e}}$ points is small. Additional high precision $B_{\mathrm{e}}$ observations are necessary to improve parameters of the magnetic variability. Open squares - Ref. 2, filled squares - Ref. 327, filled circles - Ref. 25. 
V. D. Bychkov et al.: A catalog of stellar magnetic rotational phase curves, Online Material p 47 HD223640

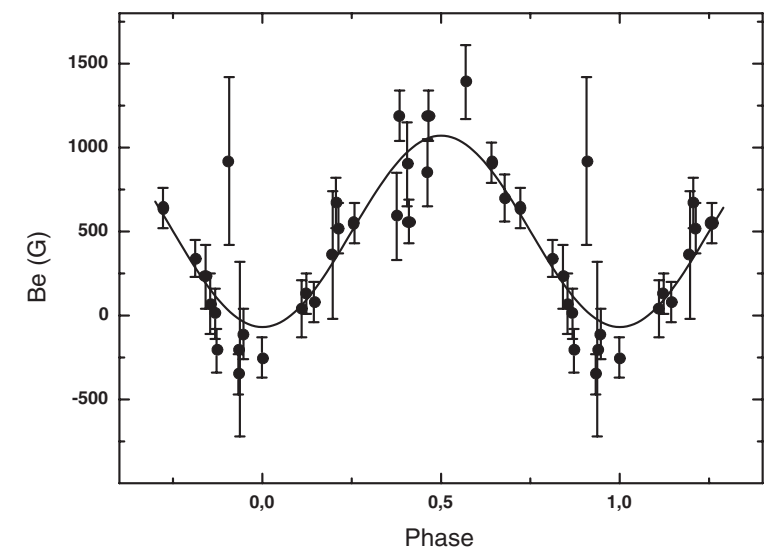

Fig. A.160. HD 223640. We used the period $P_{\mathrm{mag}}=3.735239^{\mathrm{d}}$, taken from Adelman \& Knox (1994). Magnetic field $B_{\mathrm{e}}$ was measured in metal lines, see Ref. 1, and in hydrogen lines, Ref. 224. Measurements from Ref. 1 were not used to construct phase curve due to low accuracy.

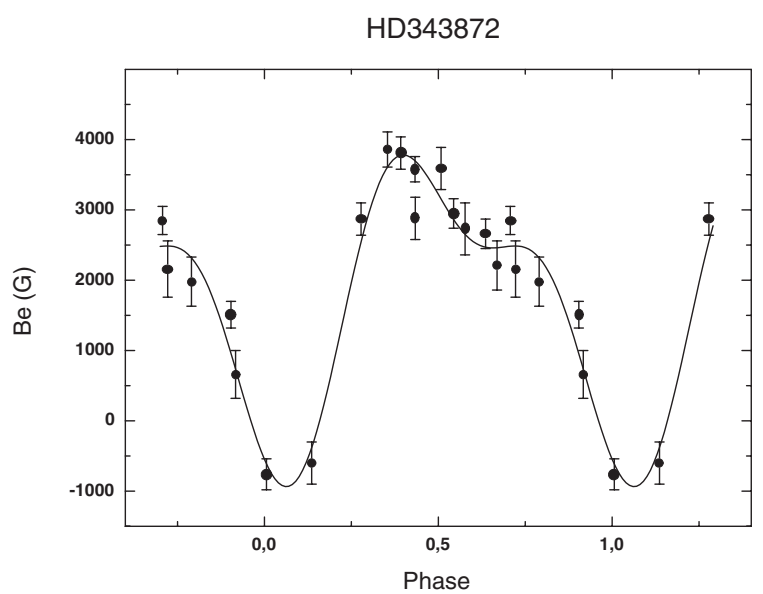

Fig. A.161. HD 343872. We determined and used here the best magnetic period $P_{\mathrm{mag}}=8.8^{\mathrm{d}}$. Magnetic field $B_{\mathrm{e}}$ of this star was measured in metal lines - Ref. 329. Phase curve obviously is a double wave. 